\title{
Evaluation of a Self-Guided Transport Vehicle for Remote Transportation of Transuranic and Other Hazardous Waste
}

\author{
RECENED \\ JUN 091997 \\ OSTI
}

P. M. Rice, Lockheed Martin Idaho Technologies Company

S. J. Moody, University of Wyoming

R. S. Peterson, RAHCO International

D. M. Lokhorst, RSA Research Ltd.

A. P. Fung, Spar Aerospace Ltd.

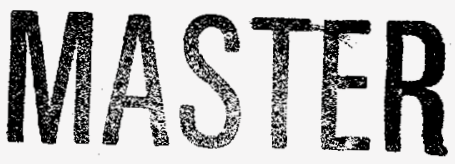




\title{
Evaluation of a Self-Guided Transport Vehicle for Remote Transportation of Transuranic and Other Hazardous Waste
}

\author{
P. M. Rice, Lockheed Martin Idaho Technologies \\ S. J. Moody, University of Wyoming \\ R. Peterson, RAHCO International \\ D. M. Lokhorst, RSI Research Ltd. \\ A. P. Fung, Spar Aerospace Ltd.
}

Published April 1997

\author{
Idaho National Engineering Laboratory \\ Environmental and Life Sciences Department \\ Lockheed Martin Idaho Technologies Company \\ Idaho Falls, Idaho 83415 \\ MASTER \\ Prepared for the \\ U.S. Department of Energy \\ Assistant Secretary for Environmental Management \\ Under DOE Idaho Operations Office \\ Contract No. DE-AC07-94ID13223
}


Evaluation of a Self-Guided Transport Vehicle For Remote Transportation of Transuranic and Other Hazardous Waste

Prepared by

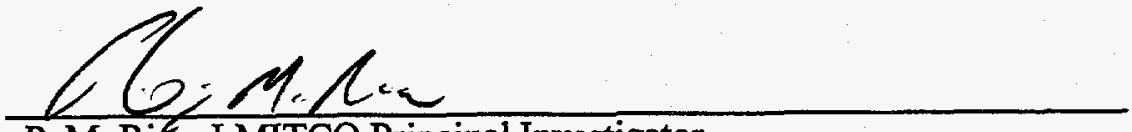

P. M. Rice, LMITCO Principal Investigator

Reviewed by

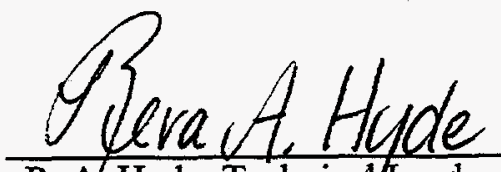

R. A. Hyde, Technical/Lead

Enyironmental Restoration Technology Department

Approved by

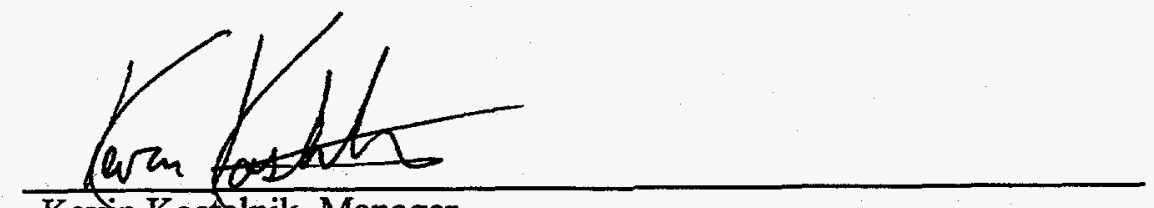

Kevin Kostelnik, Manager

Environmental Restoration Technology Department
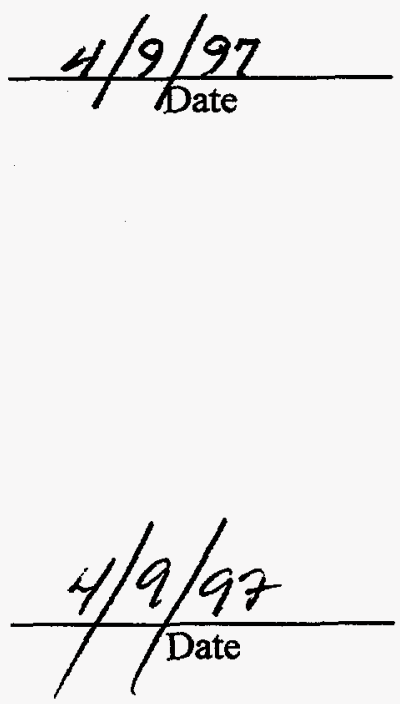


\section{DISCLAMMER}

Portions of this document may be illegible in electronic image products. Images are produced from the best available original document. 


\section{ABSTRACT}

Between 1952 and 1970 , over two million cubic $\mathrm{ft}$ of transuranic mixed waste was buried in shallow pits and trenches in the Subsurface Disposal Area at the Idaho National Engineering Laboratory's

Radioactive Waste Management Complex. Commingled with this two million cubic $\mathrm{ft}$ of waste is up to 10 million cubic $\mathrm{ft}$ of fill soil. The pits and trenches were constructed similarly to municipal landfills with both stacked and random dump waste forms such as barrels and boxes. The main contaminants are micron-sized particles of plutonium and americium oxides, chlorides, and hydroxides. Retrieval, treatment, and disposal is one of the options being considered for the waste.

This report describes the results of a field demonstration conducted to evaluate a technology for transporting exhumed transuranic wastes at the Idaho National Engineering and Environmental Laboratory (INEEL) a and at other hazardous or radioactive waste sites through the U.S. Department of Energy complex.

The full-scale demonstration, conducted at the INEEL Robotics Center in the summer of 1995, evaluated equipment performance and techniques for remote transport of exhumed buried waste. The technology consisted of a Self-Guided Transport Vehicle designed to remotely convey retrieved waste from the retrieval digface and transport it to a receiving/processing area with minimal human intervention. Data were gathered and analyzed to evaluate performance parameters such as precision and accuracy of navigation and transportation rates.

a. This work was performed under contract at the Idaho National Engineering Laboratory. In January 1997, the name of the INEL was changed to the Idaho National Engineering and Environmental Laboratory (INEEL). INEL will be used on the cover and title page until a new INEEL logo and cover is designed. INEEL will be used throughout the text of the document, except where use of INEL is historically important. 


\section{SUMMARY}

The development, demonstration, testing, and evaluation of a system for conveying buried waste was funded by the U.S. Department of Energy's Office of Technology Development through the Landfill Stabilization Focus Area (LSFA) program. The concept, design, hardware, and software for the system was developed for the LSFA program by RAHCO International in conjunction with SPAR Aerospace Ltd. and RSI Research Ltd. In the summer of 1995, a full-scale demonstration was conducted at the Idaho National Engineering and Environmental Laboratory (INEEL) to evaluate equipment performance and techniques for remotely transporting buried waste.

The objective of the demonstration was to explore the effectiveness of methods and equipment to remotely transport buried waste away from the digface. The technology evaluated in the demonstration consisted of a Self-Guided Transport Vehicle (SGTV) designed to remotely convey retrieved waste from the digface and deliver it to a handling/processing area. The SGTV represents the second generation of the development of a remote-operated transport vehicle begun in Fiscal Year (FY) 1994.

The SGTV is a remote-operated, robotic-racked vehicle capable of maneuvering on rugged terrain and in soft soils as may be expected in buried waste retrieval areas. It consists of a track-mounted, self-guided waste transportation vehicle and a remote operations support console that is used for controlling the SGTV and housing the vehicle tracking systems, communications, data logging/mass storage, and video systems. The SGTV transports loaded/unloaded transport containers and Integrated Transfer Modules (ITM). The SGTV is fully integrated with an innovative end effector/waste handling system to provide a waste transportation solution that eliminates dust generation during transportation operations.

The SGTV has two modes of operation; self-guided and telerobotic. A real-time Differential Global Positioning System/Dead Reckoning (GPS/DR) System is used to track the vehicle in the self-guided mode. The SGTV sensory suite consists of a Trimble 4000 SSI differential GPS including a base station receiver and a roving receiver, both with Trimtalk radios; a Precision Navigation TCM2 electronic compass including pitchand-roll inclinometers; a Systron Donner Rate Gyro; and dual track encoders. These devices comprise the navigation sensory system for the SGTV and are integrated via the on-board processors.

Self-guidance is provided from a computer at the control console running the Vehicle Command and Control Generator (VCCG) software. The VCCG is a software program for generating the commands to control the vehicle. The basic commands are the linear and angular rate commands for the vehicle to track a predefined path. There are also other commands such as "go", "pause", "resume" and "stop", which can be initiated by the operator or generated by the software based on the control status.

The test goals and objectives were broken down into seven areas. A list of each of these areas and a short synopsis of the test results follows. 
1. Operational and Transuranic Environment Evaluation - These test objectives focused on obtaining observations on visual dust generation, anomalous conditions, maintenance requirements, reparability, operational costs, and the overall reliability of the SGTV. The testing demonstrated that the technology embodied in the SGTV is mature enough and reliable enough that it could be applied to a production design and deployed in a full-scale transuranic waste retrieval operation with only superficial modifications.

2. Throughput - The throughput rates for the SGTV were between 43.6 and $51.7 \mathrm{yd}^{3} /$ day ( 8 hours). The length of travel required to transport the waste and the complexity of the path have the greatest effect on system throughput. The load capacity and loading and unloading time are (or should be) for the most part constant. The three test paths were designed to exercise these variables in a situation believed to be representative of a realistic scenario for a hot spot retrieval in a transuranic waste pit.

Restrictions placed on operations due to the limited availability of space for safe maneuvering, and a data network problem caused throughput rates to drop below the goal of $80 \mathrm{yd}^{3} /$ day for the typical operating scenarios simulated. Increasing the vehicle's speed to its maximum design of $5 \mathrm{ft} / \mathrm{sec}$ (linear) would provide greatly improved throughput rates. Of course, one of the most important aspects of the design of the SGTV is that since it is self-guided, multiple vehicles can be easily deployed to increase the production rate to any desired level with minimal impact to production costs.

3. Miscellaneous, Safety, and Vehicle and Control System Performance - Data from a number of tests involving safety, vehicle performance, mission planning, and the VCCG graphical user interface were gathered.

a. Safety: All of the hydraulic's enable/disable and emergency stop functions of the SGTV system design were tested and functioned as designed. There were no safety system malfunctions observed during the development or testing of the SGTV.

b. Performance: Acceleration and declaration curves were obtained to characterize the SGTV's performance. The maximum velocity of the SGTV (under the control gains set at the factory) was $6 \mathrm{ft} / \mathrm{s}$. The absolute maximum performance parameters of the SGTV were not established.

c. Mission Planning: The mission planning software was tested to determine how long it takes to define a test area and plan mission paths. A no-go zone map was created using the mission planning software per the procedure provided by Spar. The basic map for the test site had been previously loaded into the computer. Three paths were created that were designed to be representative of paths that could be used in an actual retrieval. The paths ranged from simple to difficult. The time it took to create and save these paths was 7,18 , 
and 15 minutes respectively. The goal of the design was to be able to plan or modify a path in 30 minutes or less. The software is easy to use and very effective.

d. VCCG: All of the functions on the VCCG display and command graphical user interface were tested and performed as specified. Delays in the hardware development provided time for the Spar Engineer to add functionality to and refine the interface of the SGTV so that the final product exceeded both the specification and the expectations of the operators.

4. System Integration - System integration was intended to investigate the interface between the SGTV and the other equipment that it would be working with during retrieval operations. The two pieces of equipment of interest were the remote excavator or REMEX with the innovative and effector, and the cooperative telerobotic retrieval (CTR) gantry crane. Unfortunately, the REMEX was not available at the time the actual testing took place, and the CTR gantry crane was not operational, and thus could not participate in the test. A very limited integration demonstration was performed during the LSFA exposition in August, 1995 that showed that the SGTV, REMEX, and CTR gantry crane are designed to function as a total system for retrieval and transportation of buried waste.

5. Precision and Accuracy of Navigation - Data to support calculation of the precision and accuracy of navigation were obtained by determining the difference between the actual location of the vehicle and the desired location of the vehicle. The precision specification was to track within $1 \mathrm{ft}$ of the desired path. Analysis of the data showed that the SGTV's path tracking precision varied from an average of 0.580 to $0.972 \mathrm{ft}$ depending on the path. Accuracy of tracking (absolute mean off-track error) was from -0.118 to $-0.373 \mathrm{ft}$.

6. Ultrasonic Obstacle Avoidance - The ultrasonic obstacle avoidance system (OAS) was tested to determine at what distance an obstacle is first detected, the distance an obstacle first enters the collision zone, and the distance when the emergency stop has been initiated by the obstacle avoidance system under various conditions. In all but one case, the OAS detected objects, cataloged and tracked them, and when necessary halted the vehicle a safe distance from the obstacle. When an object came within the collision zone threshold, the OAS overrode the velocity commands of the VCCG or the teleoperator and began to slow the vehicle to prepare for a safe stop. When an object no longer presented a potential hazard, the OAS relinquished control and normal operations resumed.

7. Maneuverability - Testing performed in FY-94 on the teleoperated version of the SGTV demonstrated the vehicle's basic capabilities to maneuver in rugged terrain, traverse steep slopes, and operate in 
various soil/surface conditions. The greatly improved hydraulic system and much more powerful engine implemented in FY-95 greatly improved this capability.

The equipment used for this demonstration was designed to investigate concepts for remote transportation of buried waste. Prototype hardware and low-cost components were adapted to facilitate an inexpensive, short schedule, proof-of-concept demonstration. The hardware concept and design approach supports retrieval in a transuranic environment; however, due to cost and schedule constraints, no attempt was made to provide fully industrialized equipment. There were no aspects of the equipment design that would make it difficult or prevent it from being upgraded to operate in a transuranic buried waste retrieval environment.

The data obtained in this demonstration will eventually be used by Environmental Restoration Programs during the feasibility study for parts of the INEEL Radioactive Waste Management Complex (RWMC) and, possibly, by additional programs in other locations. Environmental Restoration will also use the data to project additional technology needs for LSFA out-year planning.

\section{Addendum}

This report was initially prepared after testing concluded in October 1995 . It was originally intended to be published as an Appendix to an overall LSFA programmatic report documenting the results of the integrated demonstration for FY-95 activities. Due to development delays in other critical technologies and a lack of funding, that report was never published. This document was reworked as a stand-alone report and published in April 1997.

Since the completion of the program, RAHCO International, along with its partners SPAR Aerospace Ltd. and RSI Research Ltd., have continued development of the SGTV and the REMEX systems in the expectation of commercializing the technology and deploying it in real environmental restoration operations. Several problems and limitations of the SGTV that are discussed in the body of the report have since been corrected, and a number of performance enhancements have been incorporated into the design:

- The vehicle was shipped to RSI Research for comprehensive testing to alleviate the electronics problems encountered during the test. During this period, the electronic systems were hardened for increased reliability and durability under the harsh industrial conditions in which the SGTV operates. Two of the older Genie microcontrollers were replaced, and the mechanical relays were replaced with electronic relays to eliminate problems resulting from shock and vibration. This effectively eliminated the cause of the frequent data network crashes experienced during the demonstrations. 
- The GPS system was upgraded to a Trimble $7400 \mathrm{MSF}$. This increased the position location fix frequency to $5 \mathrm{~Hz}$ from the $1-\mathrm{Hz}$ update rate used in the demonstration. Higher-frequency updates will increase the accuracy of navigation and could reduce the reliance on dead reckoning sensors for navigation. In future iterations, this could greatly simplify the design and improve the reliability of the hardware and software.

- The vehicle tracks were overhauled to improve their performance. Track idlers were shimmed $3 / 4$ in. to reduce the shock and vibration on the vehicle and provide smoother operation.

- One of the shortcomings of the GPS system is that it can only be effectively used outdoors and only when sufficient GPS satellite coverage is available. As a backup or alternative, a laser guidance system has been incorporated to guide the SGTV indoors and during periods of poor satellite availability. The system uses a laser range-finder attached to the vehicle along with fixed reflective targets positioned about the operating area. The laser determines the real-time position of the SGTV and transmits the coordinates to the VCCG software in the same format as the GPS data. This means that the VCCG software does not have to be changed to accommodate the additional system.

- The VCCG control station has been upgraded to a Pentium 100-MHz processor for increased speed. This upgrade eased integration of the laser guidance system, provided for future growth options, and enhanced the system responsiveness. 


\section{ACKNOWLEDGMENTS}

The successful completion of this project was due to the significant contributions and efforts of the entire project team. The authors would like to express their sincere appreciation to those who contributed to the project and to the completion of this report, including, Brian Roy, Mike Gins, and Alberto Callo of RSI Research, Ltd.; Joe Sheppard, Tom Tucker, Felix Keung, John Ballantyne, and Ross Fillett of SPAR Aerospace, Ltd.; Tom Crocker, Jim Challinor, Pat Burnham, and Raymond Daigh, RAHCO International; and Brad Gardner, Bob Heard, Lewis Rounds, and Randy Eastman of Lockheed Martin Idaho Technologies. 


\section{CONTENTS}

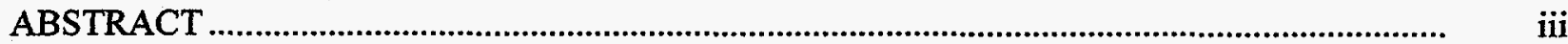

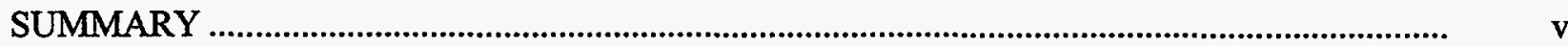

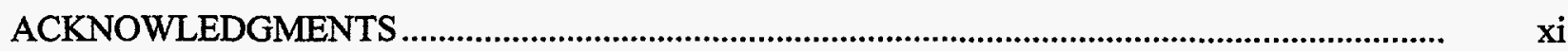

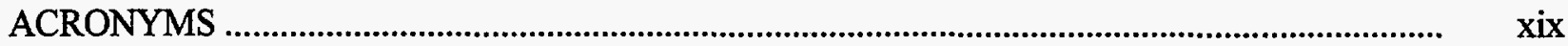

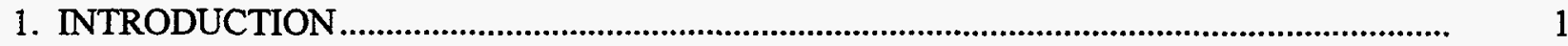

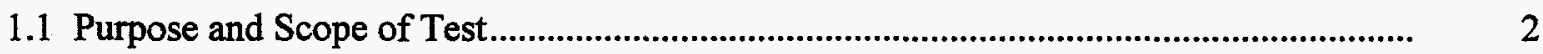

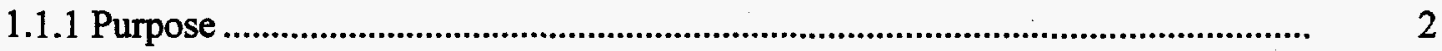

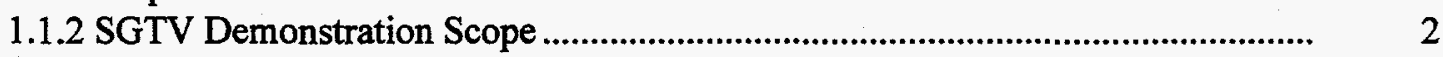

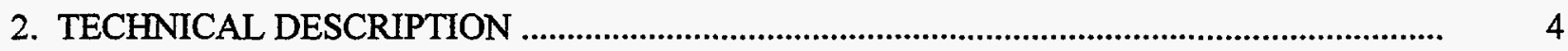

2.1 SGTV Performance/Acceptance Test Hardware Description ........................................

2.1.1 Vehicle Structure/Cradle ..................................................................................... 5

2.1.2 Power Plant/Hydraulics ....................................................................................... 5

2.1.3 Waste Transport Container................................................................................ 5

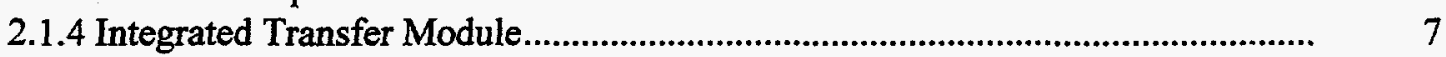

2.1.5 SGTV Control System and Electronics........................................................... 7

2.1.6 SGTV Mission Planning and Vehicle Command and Control Generator ........... $\quad 15$

2.1.7 Retrieval Support Equipment ................................................................................ 35

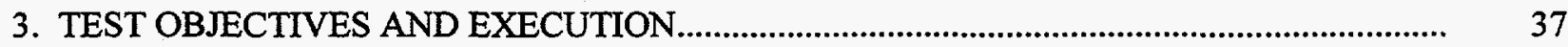

3.1 Test Objectives and Data Quality Objectives ..........................................................

3.1.1 Operational and Transuranic Environment Evaluation........................................ 37

3.1.2 Throughput ................................................................................................. $\quad 37$

3.1.3 Miscellaneous Human Interface, Safety, and Vehicle/Control

System Performance Parameters...................................................................... 38

3.1.4 System Integration............................................................................................. 39

3.1.5 Precision and Accuracy of Navigation............................................................... 40

3.1.6 SGTV Ultrasonic Obstacle Avoidance.................................................................. 40

3.1.7 Maneuverability................................................................................................... 41

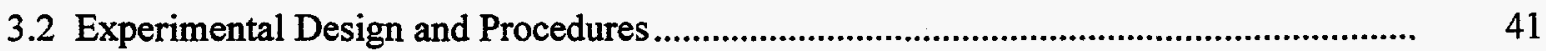

3.2.1 Test Design and Methodology .............................................................................. 41

3.2.2 Deviations from the Test Plan ...................................................................... 44

xiii 
4.1 Operational and Transuranic Environment Evaluation

4.1.1 Analysis

4.1.2 Results and Evaluation

4.1.3 Discussion/Conclusion

4.2 Throughput

4.2.1 Analysis

4.2.2 Results and Evaluation ................................................................................. 50

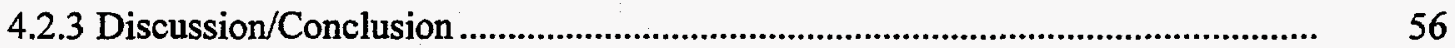

4.3 Miscellaneous HMI, Safety, and Vehicle and Control System Performance................. 57

4.3.1 Analysis

4.3.2 Results and Evaluation

4.4 System Integration

4.4.1 Analysis

4.4.2 Results and Evaluation

4.4.3 Discussion/Conclusion

4.5 Precision and Accuracy of Navigation

4.5.1 Analysis

4.5.2 Results and Evaluation

4.5.3 Discussion/Conclusion

4.6 SGTV Ultrasonic Obstacle Avoidance

4.6.1 Analysis

4.6.2 Results and Evaluation

4.6.3 Discussion/Conclusion

4.7 Maneuverability

4.7.1 Analysis

5.1 Mechanical Systems

5.1.1 Effectiveness

5.1.2 Human Factors

5.1.3 Recommended Improvements and Future Work

5.1.4 Conclusions 
5.2.3 Recommended Improvements and Future Work ............................................. 106

5.2.4 Conclusions ..................................................................................................... $\quad 107$

5.3 Mission Planning and Vehicle Command and Control Generator

\section{FIGURES}

1. Diagram of the waste retrieval process

2. The SGTV mechanical subsystem consists of a track assembly, vehicle structure/cradle, diesel power plant/hydraulic system, WTC, and guidance/control subsystems. (Photo no. 95-811-2-18)

3. The SGTV's modified structure allows it to carry two ITMs in the waste transport container and two in the ITM cradle

4. The SGTV control system

5. The SGTV control station includes the teleoperator's control station and the MP and VCCG computer (Photo no. 95-811-1-12)

6. The MP and VCCG interface directly with the telemetry and control router to take the place of the teleoperated control station for self-guided operations.

7. The SGTV path is defined by a series of waypoints that include a start point, fly-by points, pause points, and an end point

8. A coordinate system and set of units were established to define the SGTV navigation rules......

9. Mission planning no-go zone editor - map .

10. Mission planning no-go zone editor - no-go zone

11. Mission planning waypoint editor - waypoint parameters.

12. Mission planning waypoint editor - planned path.

13. VCCG graphical user interface 
14. The VCCG self-guided control module functional organization

15. The Cooperative Retrieval ITM Hoist Adapter provides a method for the gantry crane to manipulate ITMs

16. General arrangement of the Hot Spot Retrieval and Transportation

Demonstration test area at the INEEL Robotics Laboratory (NBA building)

17. Path 1 is a simple path starting at point $1 \mathrm{~S}$ and ending at point $5 \mathrm{E}$

18. Path 2 is a more complicated path, starting at point $1 \mathrm{~S}$ and ending at the same location point $10 \mathrm{E}$.

19. Path 3 is a very complicated path starting at point $1 \mathrm{~S}$ and ending at the same location point $13 \mathrm{E}$

20. The SGTV rapidly accelerates to and decelerates from its maximum speed

21. The waste transport container was modified from the FY-94

configuration to open at the end of the box to allow clearance for the

REMEX stick in the backhoe configuration

22. Data plot of the mean off-track error and regression curves of the mean

off-track error.

23. Data plot of the absolute mean off-track error (accuracy) and regression curves of the accuracy.

24. Data plot of the standard deviation precision calculations and associated regression analysis.

25. Data plot of the mean absolute deviation precision calculations and associated regression analysis.

26. Test Path 1 - Teleoperated Run 1 (TP1TO_R1).

27. Test Path 1 - Teleoperated Run 2 (TP1TO_R2)

28. Test Path 1 - Teleoperated Run 4 (TP1TO_R4).

29. Test Path 2 - Teleoperated Run 1 (TP2TO_R1).

30. Test Path 2 - Teleoperated Run 2 (TP2TO_R2)

31. Test Path 2 - Teleoperated Run 3 (TP2TO_R3).

32. Test Path 3 - Teleoperated Run 1 (TP3TO_R1)

33. Test Path 3 - Teleoperated Run 2 (TP3TO_R2) 
34. The improved mechanical systems on the SGTV greatly enhanced its capability to maneuver in steep terrain and soft, loose soil conditions (Photo nos. 95-811-1-3, 4, \&5).

35. The precision docking maneuver was performed using wooden targets to simulate an excavator or other retrieval equipment and as reference points for verifying the GPS data.

36. VCCG screen capture of test path number 1

37. VCCG screen capture of test path number 2 .

38. VCCG screen capture of test path number 3 .

39. The effect of a sustained GPS error is evident in the SGTV path trace.

40. Typical SGTV actual path superimposed on the planned path for test path number 1 .

41. Typical SGTV actual path superimposed on the planned path for test path number 2 .

42. Typical SGTV actual path superimposed on the planned path for test path number 3 .

43. Sample VCCG data screen showing off-track error, command signals, and SGTV response for test path number 1

44. Sample VCCG data screen showing off-track error, command signals, and SGTV response for test path number 2

45. Sample VCCG data screen showing off-track error, command signals, and SGTV response for test path number 3

46. Typical SGTV teleoperated path superimposed on the planned path for test path number 3 .

\section{TABLES}

1. Mechanical maintenance log for the test.

2. Times to navigate test path number 1

3. Times to navigate test path number 2

4. Times to navigate test path number 3

5. The calculated throughput rates for the SGTV for each path in Figures 17, 18, and 19.

6. Results of speed and acceleration testing 
7. The results of the testing of the system health monitoring features

8. All available emergency stop functions were tested to ensure they performed as designed

9. Mission planning with the graphical user interface of the mission planning software was very simple

10. Reference key for precision and accuracy test run data

11. Statistical analysis for test path 1 data

12. Statistical analysis for test path 2 data

13. Statistical analysis for test path 3 data

14. Reference key for the screen plots from the teleoperated precision and accuracy tests.

15. Results of OAS test series number 1

16. Results of OAS test series number 2

17. Results of OAS test series number 3

18. Results of OAS test series number 4

19. Results of OAS test series number 5

20. Results of OAS test series number 6

21. Results of OAS test series number 7

22. Results of OAS test series number 8

23. For each trial in the turning radius test, the start and end position was noted from the GPS system

24. Gross error data for the precision docking maneuver in self-guided mode is shown

25. Gross error data for the precision docking maneuver in teleoperated mode is shown

26. The results of the positioning errors for the precision docking maneuver are shown 


\section{ACRONYMS}

CTR Cooperative Telerobotic Retrieval

DR Dead Reckoning

DOE U.S. Department of Energy

DOE-ID U.S. Department of Energy Idaho Operations Office

DQO Data Quality Objective

FY fiscal year

GPS Global Positioning System

GUI graphical user interface

HDPE high-density polyethylene

HMI Human-Machine Interface

IEE Innovative End Effector

INEL Idaho National Engineering Laboratory

INEEL Idaho National Engineering and Environmental Laboratory

ITM Integrated Transfer Module

LSFA Landfill Stabilization Focus Area

MP Mission Planning

OAS Obstacle Avoidance System

OTD Office of Technology Development

PI principal investigator

PM project manager

REMEX Remote Operated Excavator

FR radio frequency

RWMC Radioactive Waste Management Complex

SDA Subsurface Disposal Area

xix 
SGTV Self-Guided Transport Vehicle

TRU Transuranic Waste

VCCG Vehicle Command and Control Generator

WTC Waste Transport Container 


\section{Evaluation of a Self-Guided Transport Vehicle For Remote Transportation of Transuranic and Other Hazardous Waste}

\section{INTRODUCTION}

Between 1952 and 1970, over two million cubic $\mathrm{ft}$ of transuranic (TRU) mixed waste was buried in shallow pits and trenches in the Subsurface Disposal Area (SDA) at the Idaho National Engineering Laboratory (INEL) Radioactive Waste Management Complex (RWMC). Commingled with this two million cubic $\mathrm{ft}$ of waste is up to 10 million cubic $\mathrm{ft}$ of fill soil.1 Approximately $6 \mathrm{ft}$ of clean overburden soil has been placed on top of the buried waste pits and trenches to isolate the waste from surface water runoff and wind erosion. The pits and trenches were constructed similarly to municipal landfills with both stacked and random dump waste forms such as barrels and boxes. The barrels are typically 55-gal drums made of metal. The boxes are typically $4 \times 4 \times 8 \mathrm{ft}$ and constructed of plywood. The drums and boxes contain wastes such as paper, sludge, metal, tools, cloth, wood, asphalt, concrete, and glass. There could also be large objects including storage tanks, vehicles, pipes, beams, and glove boxes. The main contaminants are micron-sized particles of plutonium and americium oxides, chlorides, and hydroxides. ${ }^{2}$ Retrieval, treatment, and disposal is one of the options being considered for the waste. As an aggregate, the retrieved waste will average above $10 \mathrm{nCi} / \mathrm{g}$ for the TRU elements. The waste also contains volatile organics such as trichloroethylene and carbon tetrachloride and cutting oils such as REGAL oil. The waste containers are assumed to be severely deteriorated to the point that many containers are breached, leaking, or even unrecognizable because of deterioration.

The configuration of waste described above is similar to other buried waste located at U.S. Department of Energy (DOE) sites throughout the United States. In response to the need to remediate these sites, the DOE's Office of Technology Development (OTD) has initiated a comprehensive program to develop and demonstrate advanced remediation technologies that support DOE's environmental restoration objectives. Further, OTD has initiated the Landfill Stabilization Focus Area (LSFA) Program to investigate the feasibility of using new and existing technologies for in situ treatment, removal, and treatment after removal of buried wastes.

Technologies that are scientifically sound and successfully developed within the LSFA Program will be deployed in various Environmental Restoration programs throughout the DOE complex.

In Fiscal Year (FY) 94 and FY-95 a waste transportation system was developed for the LSFA program as a component of the retrieval related technologies. The culmination of this effort was the development and demonstration of a Self-Guided Transport Vehicle (SGTV) designed for transportation of TRU waste and other hazardous materials. Together with the remote-operated retrieval equipment, it will affect the digging, dumping, and transport processes of retrieval (see Figure 1). The SGTV was developed in close coordination with other technologies, such as the remote-operated excavator (REMEX) and the Innovative End Effector (IEE), to ensure a systems design approach that will maximize the effectiveness of the combined technologies. 


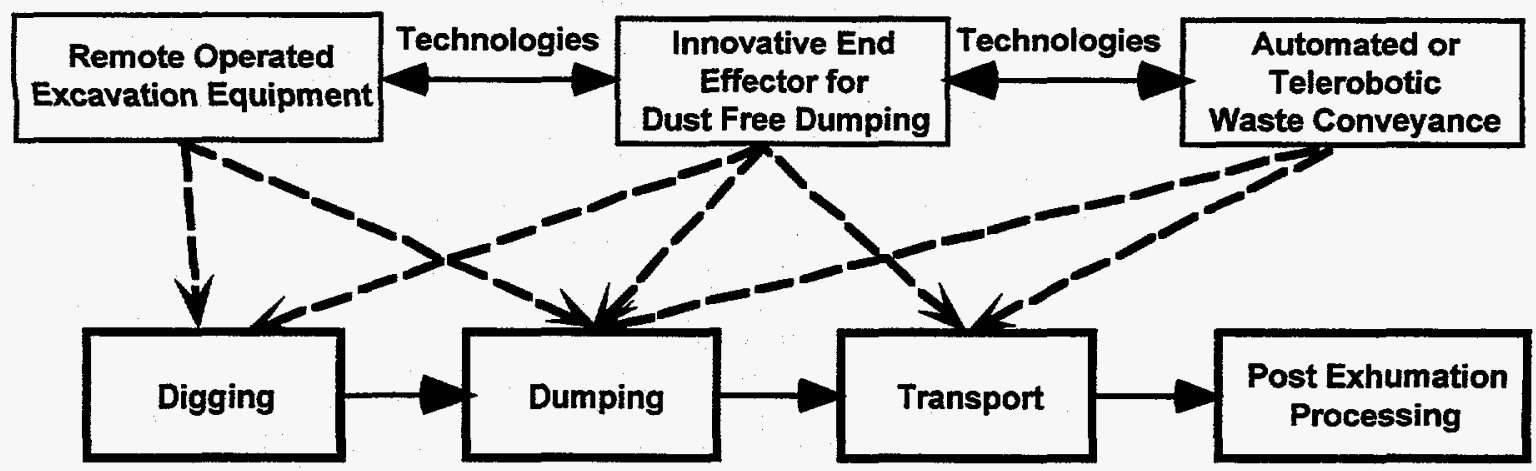

Waste Retrieval Process

Figure 1. Diagram of the waste retrieval process.

\subsection{Purpose and Scope of Test}

\subsubsection{Purpose}

The objective of the demonstration was to explore the effectiveness of methods and equipment to remotely transport buried waste after retrieval. Regardless of the excavation technique, exhumed waste will have to be transported from the retrieval area to the packaging area or directly to a treatment facility. Transportation of the retrieved waste from the digface has been performed in the past using conventional manned equipment. This report discusses the test results of a self-guided waste transportation system that began development in FY-94 as a Teleoperated Transport Vehicle (TTV). The TTV developed in FY-94 laid the foundation for the concepts that were refined and upgraded in FY-95 that culminated in the development and testing of the SGTV.

The data from this demonstration will eventually be used by Environmental Restoration for a feasibility study for parts of the RWMC at the Idaho National Engineering and Environmental Laboratory (INEEL) a and, possibly, by additional remediation efforts at other DOE locations and U.S. Superfund Sites. Data will also be used by Environmental Restoration and LSFA to project additional technology needs for LSFA out-year planning.

\subsubsection{SGTV Demonstration Scope}

The SGTV demonstration was to be conducted in two phases. The first phase of testing involved SGTV acceptance and integration testing and was to be the primary performance data gathering test. The second phase of the demonstration was to be held in conjunction with the

a. This work was performed under contract at the Idaho National Engineering Laboratory. In January 1997, the name of the INEL was changed to the Idaho National Engineering and Environmental Laboratory (INEEL). INEL will be used on the cover and title page until a new INEEL logo and cover is designed. INEEL will be used throughout the text of the document, except where use of INEL is historically important. 
LSFA integrated demonstration at the INEEL in Idaho Falls, Idaho. The objective of the LSFA integrated demonstration at the INEEL was (1) to evaluate the technical performance of the SGTV (when integrated with the IEE and REMEX) to remotely retrieve a simulated buried waste hot spot in a test pit constructed at the INEEL, and (2) to demonstrate the SGTV's capabilities to the LSFA, DOE personnel, and the public. The SGTV testing objectives included:

- Determine the rate of waste transportation

- Demonstrate the ability of the SGTV to maneuver and to negotiate rugged terrain

- Demonstrate the effectiveness of the remote control system and determine the precision and accuracy of navigation

- Demonstrate proper function of all the SGTV control systems, including, but not limited to collision avoidance, emergency stop, mission planning, hydraulic mechanism operations, and teleoperations.

- Demonstrate integrated performance with the Cooperative Telerobotic Retrieval (CTR) Gantry Crane and the REMEX.

Due to circumstances, it was not possible to complete all of the test objectives that were planned. Schedule delays and prior commitments forced an unanticipated change of venue, making it impossible to execute some of the test sequences. Availability of the CTR and REMEX was severely restricted allowing only very limited integration testing. 


\section{TECHNICAL DESCRIPTION}

\subsection{SGTV Performance/Acceptance Test Hardware Description}

The SGTV was designed and provided by RAHCO International in conjunction with Spar Aerospace Ltd., and RSI Research Ltd. It consists of a track-mounted, self-guided waste transportation vehicle and a remote operations support console that is used for controlling the SGTV and housing the vehicle tracking systems, communications, data logging/mass storage, and video systems. The SGTV transports loaded/unloaded transport containers and Integrated Transfer Modules (ITM). The SGTV is fully integrated with an innovative end effector/waste handling system to provide a waste transportation solution that significantly reduces dust generation during transportation operations. The SGTV as shown in Figure 2 is designed as a multipurpose vehicle. First, it serves as a transfer station at the excavation site by (1) supporting the transport container, (2) opening, closing, and locking the container lid, and (3) providing a staging platform for the empty ITMs. Second, it conveys full containers and ITMs from the excavation location to the dumping/unloading location. Third, it conveys the empty containers and ITMs from the storage area to the excavation site.

The SGTV control system was built around the hardware/software successfully demonstrated by the LSFA program in FY-94. Upgrades to the mechanical subsystems and control system to achieve self-guidance included:

- Modifications to the vehicle structure to accommodate two ITMs and position the Waste Transport Container (WTC) to allow placement of the ITM by a backhoeconfigured end effector

- Upgrading the power plant/hydraulics to increase horsepower and provide easier maneuvering and vehicle control

- Modifying the WTC to accommodate two ITMs

- Integration of a Global Positioning System (GPS)/Dead Reckoning (DR) control scheme

- Integration of a computer-based controller with mission planning and tracking software

- Inclusion of additional acoustic sensors tuned, positioned, and upgraded to existing software to provide collision avoidance capability

- Upgrading the operator workstation to provide an easy-to-use graphical user interface (GUI) for SGTV path planning, cueing camera views, and displaying operator required data and system health status

- Improving feedback on the health of the SGTV during prolonged operations. 


\subsubsection{Vehicle Structure/Cradle}

A main frame connects the tracks and supports the loads. One track is mounted rigidly to the frame while the second track is connected by a horizontal pivot pin. This minimizes racking of the frame as the SGTV travels over irregular terrain.

The SGTV is designed to travel at speeds up to $300 \mathrm{fpm}$. Flat grouser pads on the tracks allow the SGTV to operate on asphalt or in soil. The track grousers also help to minimize ground pressure, dust generation, and soil plowing during turning.

A simulated roller table is located on top of the main frame. On an operational transporter, the rollers will be powered and used for rapid off-loading of filled containers and onloading of empty ITMs and containers.

Located at one end of the SGTV is a cradle for holding two ITMs. The cradle is a three-sided steel frame supported by eight large springs and two cradle lock arms. The cradle and spring structure is attached to the SGTV frame with steel cables. This spring- and cablemounted structure allows the ITM to self-align with the end effector during the ITM-end effector attachment operation. The cradle lock arms provide stability to the cradle during transportation to reduce excessive bouncing of empty ITMs (Figure 3).

\subsubsection{Power Plant/Hydraulics}

The SGTV is powered by a 100 -hp, water-cooled, John Deere diesel engine, with a belt-driven $24 \mathrm{Vdc}$ generator providing electrical power for the control system. The engine is directly coupled to two Rexroth Hydrostatic drives that power the SGTV's two tracks. The engine is also coupled to a variable displacement hydraulic pump that powers the SGTV's various hydraulic components.

\subsubsection{Waste Transport Container}

The 9,000-lb capacity WTC receives retrieved waste in the form of filled ITMs, $4 \times 4 \times 8$ $\mathrm{ft}$ boxes, and all other irregularly-shaped large objects. The WTC is a steel structure approximately $5 \mathrm{ft}$ wide $\times 5 \mathrm{ft}$ high $\times 9 \mathrm{ft}$ long, weighing approximately $2,800 \mathrm{lb}$ (Figures 2 and $3)$.

The rectangular lid is connected to the base structure by a simple hinge assembly. The lid is opened by a lift mechanism to provide access for easy placement of the ITMs or other waste forms. A pocket located in the bottom of the container is configured and sized to provide placement of two ITMs into the WTC. Dust seals, located on all sealing surfaces, are provided to contain the dust generated during transportation. The WTC is also provided with lift pockets to facilitate handling with a standard forklift.

A spring-loaded latch locks the lid to secure it during handling, thus preventing inadvertent opening of the container. A second latch mechanism is provided to lock the WTC to the SGTV, thus preventing detachment of the WTC from the SGTV during transporting. 


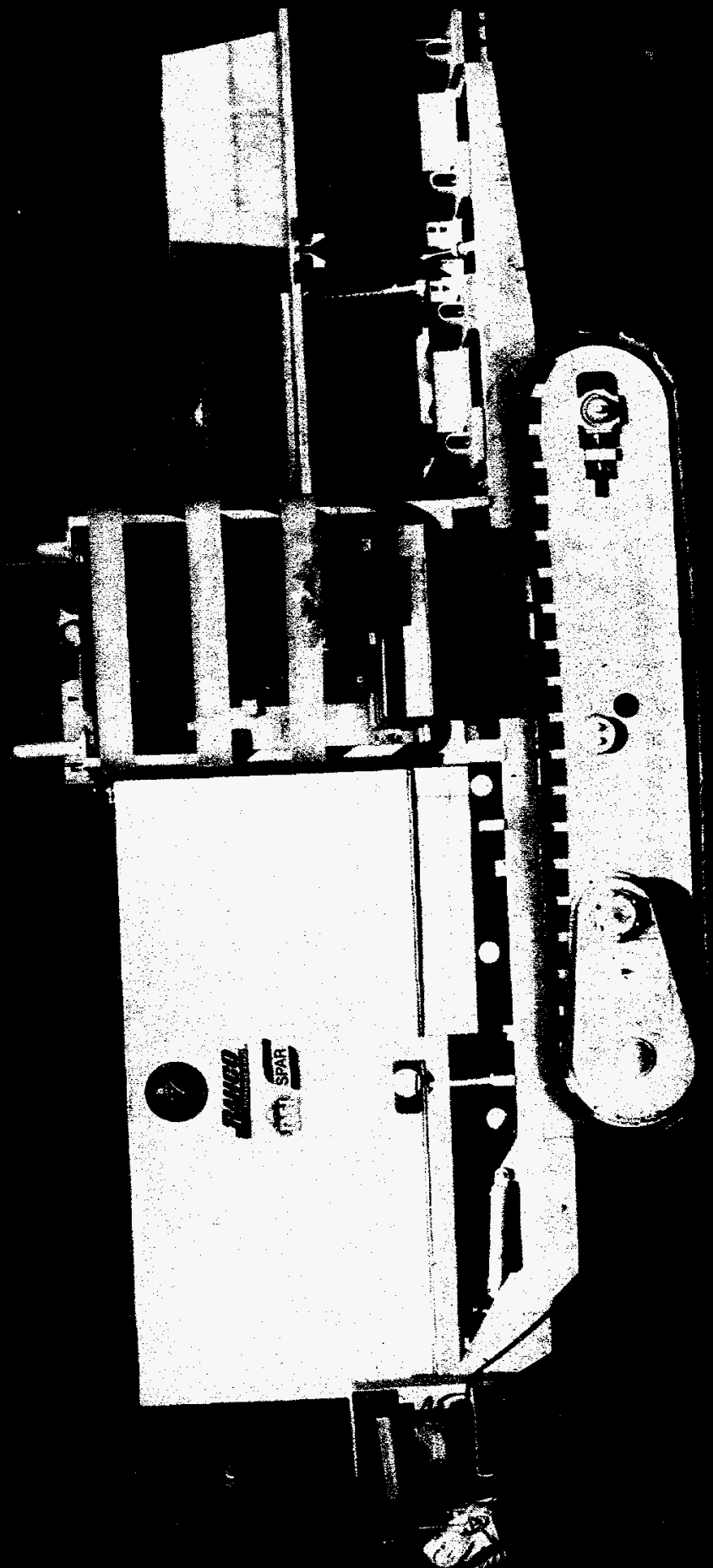

준

娄

要焉

के

政

¿

D

क्ष

क 응 용요 o 氖 를류 둥

品 a $\supset$ ธี خิํㅡㅇ 늘 옹 옹 을 $\approx$ 넝 5 क . 可 8 ठ 늘 풍 พิ 政? 릉 证 . 政 들 के 政 के 定究 क के (잉 홉음 ㄴㄹㅇ 0 ? 은 를 하믈 प्र 


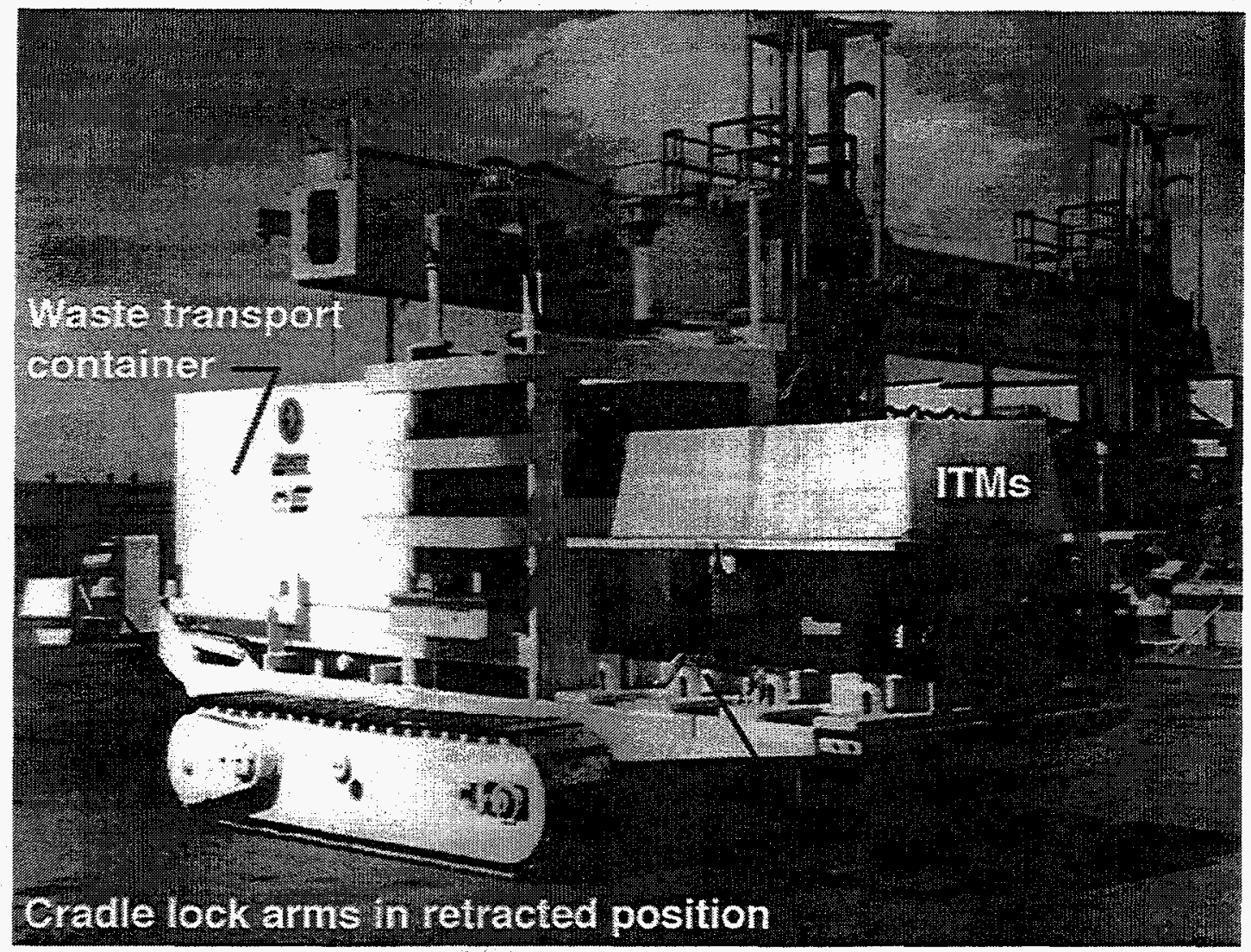

Figure 3. The SGTV's modified structure allows it to carry two ITMs in the waste transport container and two in the ITM cradle.

\subsubsection{Integrated Transfer Module}

There are two styles of ITMs, one style is a welded steel structure having inside dimensions of approximately $36.5 \mathrm{in}$. wide $\times 26 \mathrm{in}$. long $\times 36 \mathrm{in}$. deep. The other style is a molded high-density polyethylene (HDPE) plastic structure having inside dimensions of approximately 37.25 -in wide $\times 27.75$-in. long $\times 36$-in deep. They each hold $0.75 \mathrm{yd}^{3}$ of soil and waste or one 55-gal drum. Flared skirts around the upper portion are similar to that on the innovative end effector (IEE) bucket assembly and assist the operator in mating the ITMs with the bucket assembly. The tapered bottoms assist the operator in placing the ITMs into the transport container pocket (Figures 2 and 3). Slots in the ITM's top flanges allow latches on the IEE to be engaged and lock the unit to the bucket assembly.

\subsubsection{SGTV Control System and Electronics}

The SGTV has two modes of operation: self-guided and telerobotic. A real-time Differential Global Positioning System/Dead Reckoning System (GPS/DR) is used to track the vehicle while in the self-guided mode. A control system diagram for the SGTV is shown in Figure 4. 


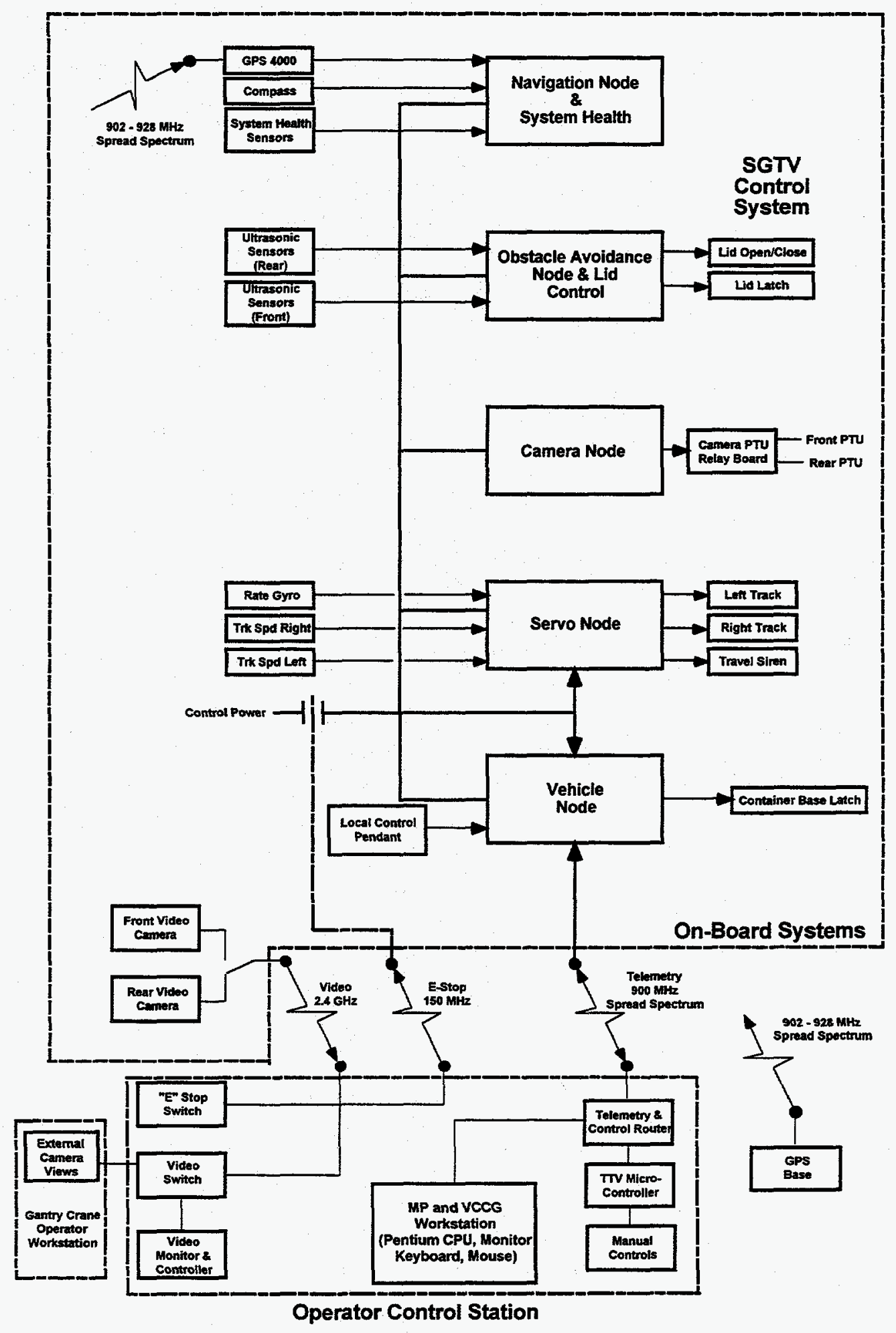

Figure 4. The SGTV control system. This system incorporates improvements and enhancements, which build upon the base system developed in FY-94 for the teleoperated transport vehicle. 
2.1.5.1 Sensors and Electronics. The SGTV sensory suite consists of a Trimble 4000SSI differential GPS including a base station receiver and a roving receiver, both with Trimtalk radios; a Precision Navigation TCM2 electronic compass including pitch-and-roll inclinometers; a Systron Donner Rate Gyro; and dual track encoders. These devices comprise the navigation sensory system for the SGTV and are integrated via the on-board processors.

A spread spectrum radio modem (Proxim Proxlink/XR) provides the telemetry communication link. A redundant, dedicated radio receiver (Remtron RCS10) controls the emergency stop system.

A control pendant is provided to connect to the vehicle, and allow local operation (independent of the Teleoperator's Station). The pendant provides an emergency stop switch, a two axis joystick for track control, and switches to activate the lifting and latching functions.

The control system computer and related electronics are housed in an environmental enclosure. A key-lock power switch, an emergency stop switch, and the master Local/Remote control switch is located on the enclosure.

The electronic control system for the SGTV comprises five RSI Research Ltd. microcontroller units. Each of these units has a 16-MHz, 16-bit Intel 80C196KR microcontroller, as well as eight channels of on-board analog signal input, ten high-speed digital power outputs, and support for a keypad and display. The five microcontrollers communicate with each other over an RS-485 serial network. Functionality of the control system is divided between these microcontrollers as follows (reference Figure 4):

\section{Microcontroller 1 - Vehicle Node}

The Vehicle Node is the master node of the on-board control system. It handles telemetry communication with the operator's control station, as well as inputs from the control pendant. The Vehicle Node handles all $\mathrm{I} / \mathrm{O}$ and control functions dealing with the waste container and module, including lifting arm and latch. Sequencing algorithms for these functions are implemented in this controller.

Sensory Inputs: on-board joysticks, rate gyroscope, limit switches

Control Outputs: hydraulics enable, container base latch control valve

\section{Microcontroller 2 - Servo Node}

The Servo Node handles all $\mathrm{I} / \mathrm{O}$ and control functions dealing with the tracks on the vehicle. Servo control algorithms are implemented in this controller.

Sensory Inputs: dual-track speed encoders

Control Outputs: proportional track control valves, warning siren, cradle locks 


\section{Microcontroller 3 - Camera Node}

The Camera Node handles all control $1 / O$ and control functions dealing with the cameras and pan and tilt units on the vehicle. (The video signal from the camera is transmitted by a separate system described in Section 2.1.5.4.)

Control Outputs: camera select relay, pan motors, tilt motors, zoom motors, focus motors

\section{Microcontroller 4 - Obstacle Avoidance Node}

The Obstacle Avoidance Node handles control and sensing of ranges reported by the Obstacle Avoidance System (OAS) transducers. Verified obstacles are classified as to importance and a diminishing maximum vehicle velocity is enforced on the servo control system as obstacles near the SGTV.

Sensory Inputs: ultrasonic sensor array ( 2 banks)

Control Outputs: lid open/close, lid latch

\section{Microcontroller 5 - Navigation Node}

The Navigation Node handles input from all velocity sensors and determines a dead reckoned (DR) position of the SGTV in local coordinates based upon both on-board sensors and the external absolute position reported by the differential GPS. The dead reckoned position is reported to the vehicle node and thereby to the Vehicle Control Command Generator (VCCG) as part of the telemetry uplink.

Sensory Inputs: GPS position and heading, compass heading, pitch and tilt

2.1.5.2 Control Implementation. The actual control is implemented through a supervisory position/orientation loop and an inner regulatory rate control loop. The rate loop is formed by the SGTV's on-board controller. This controller drives the SGTV's left and right track hydraulic motors in response to supervisory signals for commanded linear/angular speed.

Two additional modules on the SGTV on-board control system support vehicle positioning through GPS and dead reckoning:

- Dead Reckoning. Using sensor input from the gyro, compass, and track shaft encoders, a current $D R$ velocity and direction is calculated and a DR position determined built on the latest position out of the Position Determination Filter. Redundant sensor inputs are filtered before being used in the dead reckoned position calculation.

- Position Determination Filter. This module corrects the differential GPS reading delivered at $2 \mathrm{~Hz}$ by the Roving GPS for latency. The one second latent GPS position is adjusted using the dead reckoned position to estimate the actual GPS position of the vehicle. 
A final best estimate of the position of the vehicle is obtained by combining weighted GPS and DR positions into a single current position. This is performed using predetermined confidence levels for each type of position that take into account known measurement errors. The final position estimate is transmitted to the VCCG as part of the telemetry stream to the base station.

The supervisory position/orientation loop is driven by the VCCG, a new workstation-based module which supplies telemetry downlink rate commands to the SGTV's onboard controller. The position/ orientation loop is closed through sensing of SGTV position and orientation via the on-board DR system. The details of the VCCG design and implementation are discussed in Section 2.1.6.

2.1.5.3 Automatic Collision (Obstac/e) Avoidance. The automatic collision avoidance system for the SGTV is based on the use of acoustic range sensors. These sensors are used to detect objects within approximately $35 \mathrm{ft}$ of the SGTV to provide collision avoidance during normal self-guided operations and/or teleoperation. They can also be used to provide location referencing for parking/docking at a predetermined location.

Echoes from any target must persist over a predetermined sample period before they can be considered obstacles. Because of the difficulty of resolving the bearing of obstacles, they are considered to extend as a wall across the forward sonic zone at right angles to the direction of travel. No warning of echoes from any target will occur until an obstacle has been verified. Similarly, no detected obstacle will be abandoned until it has disappeared for the same sample period. Three sonic zones are considered to exist ahead of the vehicle:

Detection zone - an outer area where obstacles are registered. The operator is alerted, but no action is taken.

Collision zone - the inner area where the operator is alerted to the presence of an obstacle and its distance from the SGTV. In this zone, the vehicle's speed is reduced as it approaches the obstacle.

Emergency - Stop (E-Stop) zone - the area in which the OAS system commands the vehicle to stop within a safe distance of the obstacle. The e-stop zone distance can be set by the operator. This parameter is based on the system latency, operating speed of the vehicle, and the OAS reporting frequency.

The E-stop zone normally extends about $5 \mathrm{ft}$ in front of the sensors on the vehicle. Objects inside this zone cause a zeroing of the desired velocity to the servo module and shut off of the hydraulics. Under certain circumstances, it is necessary for the SGTV to approach well-known objects such as the gantry crane, loading docks, or an excavator. In these cases, the obstacle avoidance system will prevent collision but be tolerant enough to allow a slow-speed approach to the obstacle.

2.1.5.4 Cameras/Video. The television system uses two color cameras mounted in weatherproof enclosures. Each color camera has a pan and tilt unit. At any time, one video signal is transmitted; a video switching relay on-board the vehicle selects between the two camera views. A single modulator and power amplifier (Dynatech GN-1000TX) and one antenna transmit the video signal. 
At the Mobile Remote Operations Center, a single demodulator (Dynatech GN-1000RX) feeds one 14-in. color monitor. (Controls for the pan and tilt units are provided in the Teleoperator's Station as described in Section 2.1.5.6, "Teleoperator's Station").

2.1.5.5 Control Software. The on-board software is distributed among the network of five microcontrollers. All software was written in $C$ language, and operates on the Tempo real-time operating system from RSI Research.

The SGTV on-board system has four modes of operation. It switches between these modes of operation based on operator commands over the telemetry system. Descriptions of each mode are given below.

\section{Mode 1: Direct (Open Loop) Track Control}

This is the only mode of control available for the on-board pendant. It is also available for use during remotely controlled operation if Mode 2 control (described below) is not desired.

Sensory feedback is ignored. The tracks are controlled in an open loop mode from the joystick.

\section{Mode 2: Telerobotic (Closed Loop) Track Control}

This mode of operation is intended for use during normal travel, in teleoperated control.

Closed loop control of the tracks is provided. The vehicle responds to continuously updated desired linear and angular velocity commands received via telemetry. The on-board track speed sensors and the rate gyroscope are used for feedback to the servo control system.

In this mode, the ultrasonic range sensor array is monitored continuously. If the sensors indicate that objects are within preset collision avoidance range, then the control system will slow the vehicle. If objects are within a preset emergency stop range, then the control system will stop the vehicle. The status of the collision avoidance system is continuously sent back to the operator station.

\section{Mode 3: Lid/Latch Control}

This mode is intended for use during loading and unloading, while the vehicle is stationary. Commands from the operator (via telemetry) initiate the individual functions to be operated (e.g., lid open). The on-board control system monitors the available limit switches for feedback to the operator.

\section{Mode 4: Self-Guided}

This mode of operation is similar to Mode 2 except that the command signal is issued by the VCCG automatically instead of the teleoperator with the joystick (see Section 2.1.6.3 for details).

In all four modes of operation, the position determination software is active. This software determines a DR position of the SGTV in local coordinates based upon both on-board sensors and the external absolute position reported by the differential GPS positioning system. The DR position is reported to the VCCG as part of the telemetry uplink. 
Operation of the SGTV can be carried out using Modes 1 and 2 at the remote operator's station or independently at the SGTV itself using the pendant controls. An emergency stop button is also provided on the SGTV.

The self-guidance mode was used for the waste retrieval demonstration; however, if teleoperation is desired the operator should control the vehicle in the telerobotic Mode 2 using the single joystick for travel control and the forward or rearward cameras for visibility.

A graphical overlay can be displayed on the operator control station monitor. The overlay provides the operator with an image to track the target into. Boundary conditions on the overlay provide the operator with alignment and approximate ranging data, confirming the data provided by sensors and displayed on the panel LCD. From this, a skilled operator can determine the distance and attitude of the vehicle with respect to the targets and stop within $\pm 1 \mathrm{ft}$ of the required location in teleoperated mode.

2.1.5.6 Teleoperator's Station. The teleoperator's control station (Figure 5) consists of a microcontroller based computer system, a control panel, and a telemetry radio. (TV monitors are part of the Television System previously described). The control panel contains the following switches and indicators:

- A single two-axis joystick for track control in either Direct Track or Telerobotic Track operation described above

- A mode select switch allowing Off / Direct Track / Telerobotic Track / Lid \& Latch / Self-guided control selection

- Camera select, pan, tilt, and zoom switches

- Switches for lid open/close and latch/unlatch

- Hydraulics off switch

- $\quad$ Keypad for setting parameters

- LCD display for showing messages and status information.

A spread spectrum radio modem (Proxim Proxlink/XR) is used for radio telemetry. A redundant, dedicated radio transmitter (Remtron RCS10) is used for the emergency stop system.

2.1.5.7 System Health Monitoring. Sensors are located on the SGTV to keep the operators advised of vehicle status and to monitor important system health parameters. System health monitoring includes: engine oil pressure, engine temperature, battery level (low voltage indication), hydraulic oil temperature, on-board electronics temperature, container latch state, and container lid state. In addition, the VCCG monitors status of the GPS, electronic compass, data network, and telemetry. 


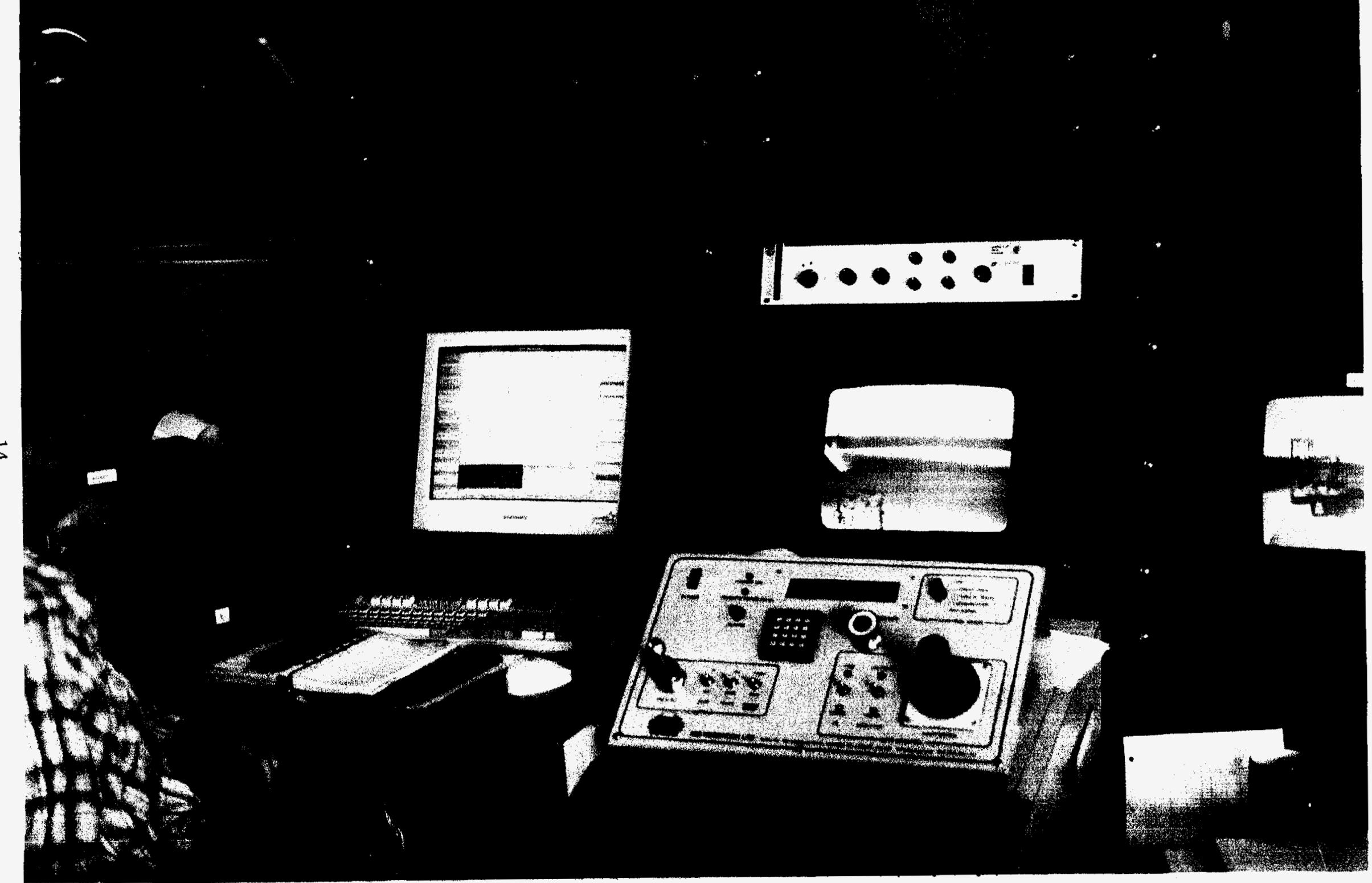

Figure 5. The SGTV control station includes the teleoperator's control station and the MP and VCCG computer (95-811-1-12). 


\subsubsection{SGTV Mission Planning and Vehicle Command and Control Generator}

The Mission Planning (MP) and Vehicle Command and Control Generator (VCCG) are the two high-level control functions of the SGTV. MP is used to develop a computer graphics map of the worksite and to build no-go zones (obstacles) on the map. The map can then be used as a background to plan paths for the vehicle to run. MP is executed in non-real time and is designed for an operator to create the no-go zones and paths. The VCCG is executed in real time. It includes a kinematic simulation capability for pre-run analysis. Its main function is to execute the paths planned in MP. The VCCG also has a user-friendly Graphical User Interface (GUI) which allows the operator to interact with the self-guided control system.

The architecture of the MP and VCCG is shown in Figure 6, and the details of the MP and VCCG will be described in the next two subsections.

2.1.6.1 Computer System. The hardware of MP and VCCG consists of a Pentium computer with:

Intel Pentium $75 \mathrm{MHz}$ central processor

- $\quad 256 \mathrm{~KB}$ cache memory

- 16 MB RAM

- $\quad 540 \mathrm{MB}$ Western Digital hard disk

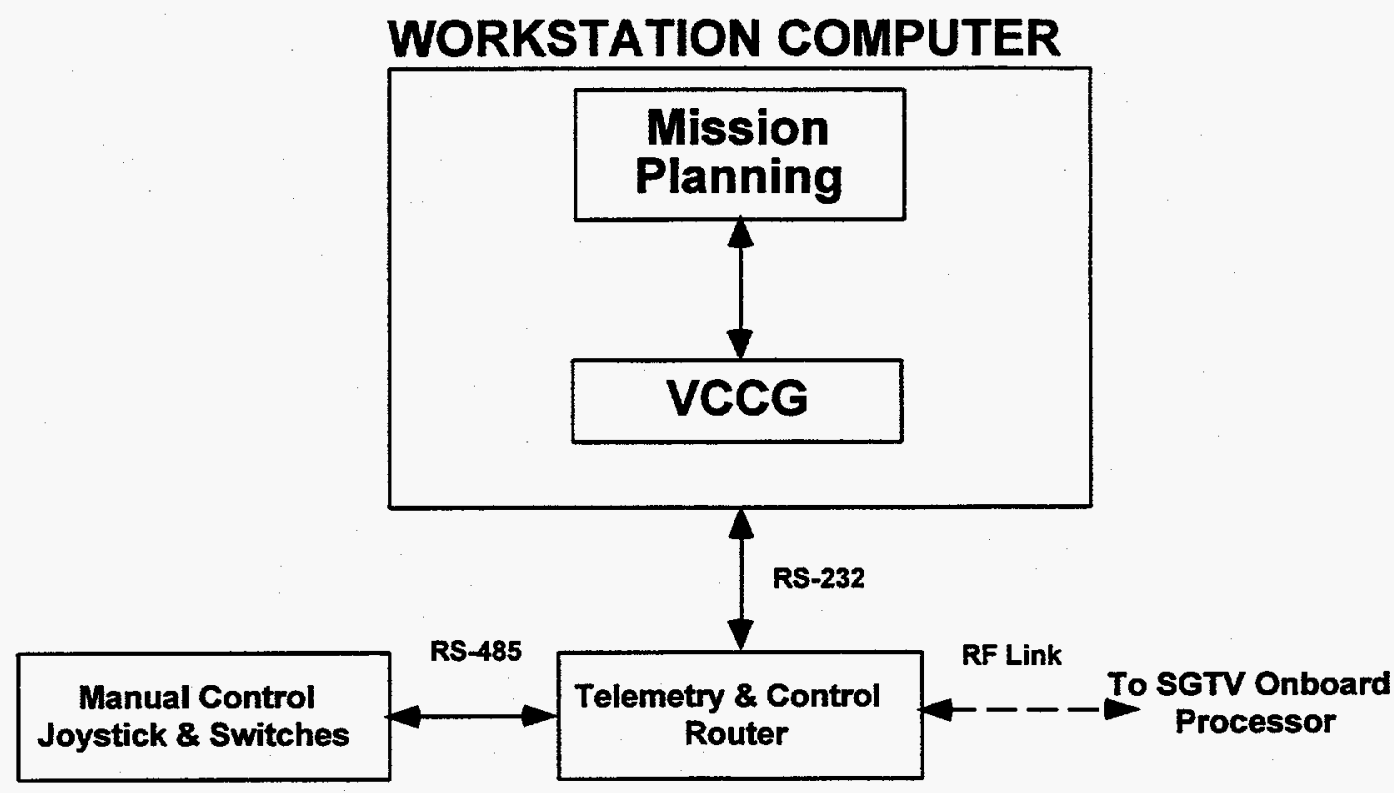

Figure 6. The MP and VCCG interface directly with the telemetry and control router to take the place of the teleoperated control station for self-guided operations. 
- $\quad 420 \mathrm{MB}$ tape drive

- 17-in. Sony monitor

- ATI MACH64 video card with 2 MB VRAM

- $\quad 216550$ serial ports, 1 parallel port

- 101 keyboard and a Logitech mouse.

The computer is linked with the Telemetry and Control Router of the SGTV through a RS-232C serial link. The software for developing the MP and VCCG is Visual Basic, which runs on the Microsoft Windows 3.11 platform.

2.1.6.2 Mission Planning. Mission Planning is a software program that allows an operator to create the map of a worksite, specify no-go zones (obstacles) on the map with buffer zones, and plan paths for the vehicle to follow. It can also be used to perform path analysis, such as collision detection. The planned paths can be stored and used by VCCG later.

2.1.6.2.1 Main Features-The following are the three main features of MP:

1. The map of the worksite uses a bitmap format, which can be imported from another source. A local grid coordinate system is superimposed on the map to provide precise dimensional control for creating no-go zones and planning paths.

2. MP allows the operator to draw no-go zones on the map. The no-go zones are areas that the vehicle cannot travel in. There are two types of shapes for defining the Nogo zones, circular and rectangular. The radius of a circle and the sides of a rectangle are defined by the operator. The operator can also specify a buffer zone around the no-go zones. The no-go zones can be stored and modified later as required. The details of creating no-go zones are described in subsection 2.1.6.2.3.

3. When the operator is ready to plan a mission, he first retrieves a map with no-go zones defined. Either the mouse or keyboard may be used to enter the waypoints of a path. Except for the start point and the end point, the intermediate points may be defined as pause points, flyby points, or smooth flyby points. The details of path planning are described in subsection 2.1.5.2.4.

2.1.6.2.2 Path Definition-This section defines the navigation path for the SGTV. Before explaining the path definition, it is essential to understand the following terminology:

Path: The route that the vehicle travels from a start point to an end point. It is composed of waypoints and line segments.

Waypoints: $\quad$ All points on a path (two consecutive waypoints form a straight path) 
Start Point:

End Point:

Flyby Point:

Smooth Flyby Point:

Pause Point:

Target Point:

Washin Circle:

Washout Circle:

Flyby Circle:

Pause Circle:

Position Hold Circle:

Off-track Error:

Heading Error:

Rate Command:

Actual Rate:
The first point of a path

The last point of a path

A waypoint for transitioning the vehicle from one straight path to the next straight path. Any off-track error will be corrected after the initial heading has been modified.

A waypoint for transitioning the vehicle from one straight path to the next straight path by modifying the initial heading and any offtrack error simultaneously.

A waypoint where the vehicle is paused. The vehicle will continue tracking upon receiving the "Resume" command from the operator.

A final waypoint where the vehicle is currently commanded to reach.

A circle around a point where the vehicle starts from zero rate. The vehicle rate command is ramped up to the rate limit in a given time, which is a function of the rate limit and the washin radius.

A circle around a target point (flyby, pause, end points). When the vehicle reaches the circle, the commanded rate is reduced gradually until it reaches a smaller circle. Then the commanded rate is set to zero. The smaller circle is called the Flyby circle for a Flyby point, Pause circle for a Pause point and Position Hold circle for an end point.

A circle around a Flyby Point. When the vehicle reaches the circle, it will travel to the next target point immediately.

A circle around a Pause Point. When the vehicle reaches this circle, it will stop automatically and will continue along the remaining path upon receiving the "Resume" command.

A circle around the End Point. When the vehicle reaches this circle, it will stop automatically and the self-guided control is completed.

Deviation of the vehicle position from a planned path.

Deviation of the vehicle heading from the desired heading.

The linear and angular rates generated by the VCCG

The sensed linear and angular rates of the vehicle.

A path consists of a sequence of waypoints as shown in Figure 7 and the coordinate system for defining the path is depicted in Figure 8. The first point is the planned starting 
position (start point) of the vehicle and the last point is the planned final destination (end point). Intermediate points can be defined between the start point and the end point. Each intermediate point must either be a "flyby" point or "pause" point. If the flyby radius of a flyby point is greater than $2 \mathrm{ft}$, then the flyby point is treated as a smooth flyby point automatically.

Each point is assigned with a circle relating to the function of that point. The start point is assigned with a "washin circle," which is for increasing the linear rate command gradually to reduce overshoots. The "washin circle" is also used at any point where the vehicle starts from zero rate. The flyby point is assigned with a "flyby circle" to reduce tracking time. The "pause point" is assigned with a "pause circle" to avoid hunting an accurate position for a temporary stop. The "end point" is assigned with a "position hold circle" relating to the required positioning accuracy. Each intermediate point and the end point is associated with a washout circle. When the vehicle reaches the "washout circle," the linear rate command will be reduced gradually until it reaches the circle set for that point. The vehicle will change course in a "flyby circle," whereas in a "pause circle" or "position hold circle," it will stop via a zero rate command.

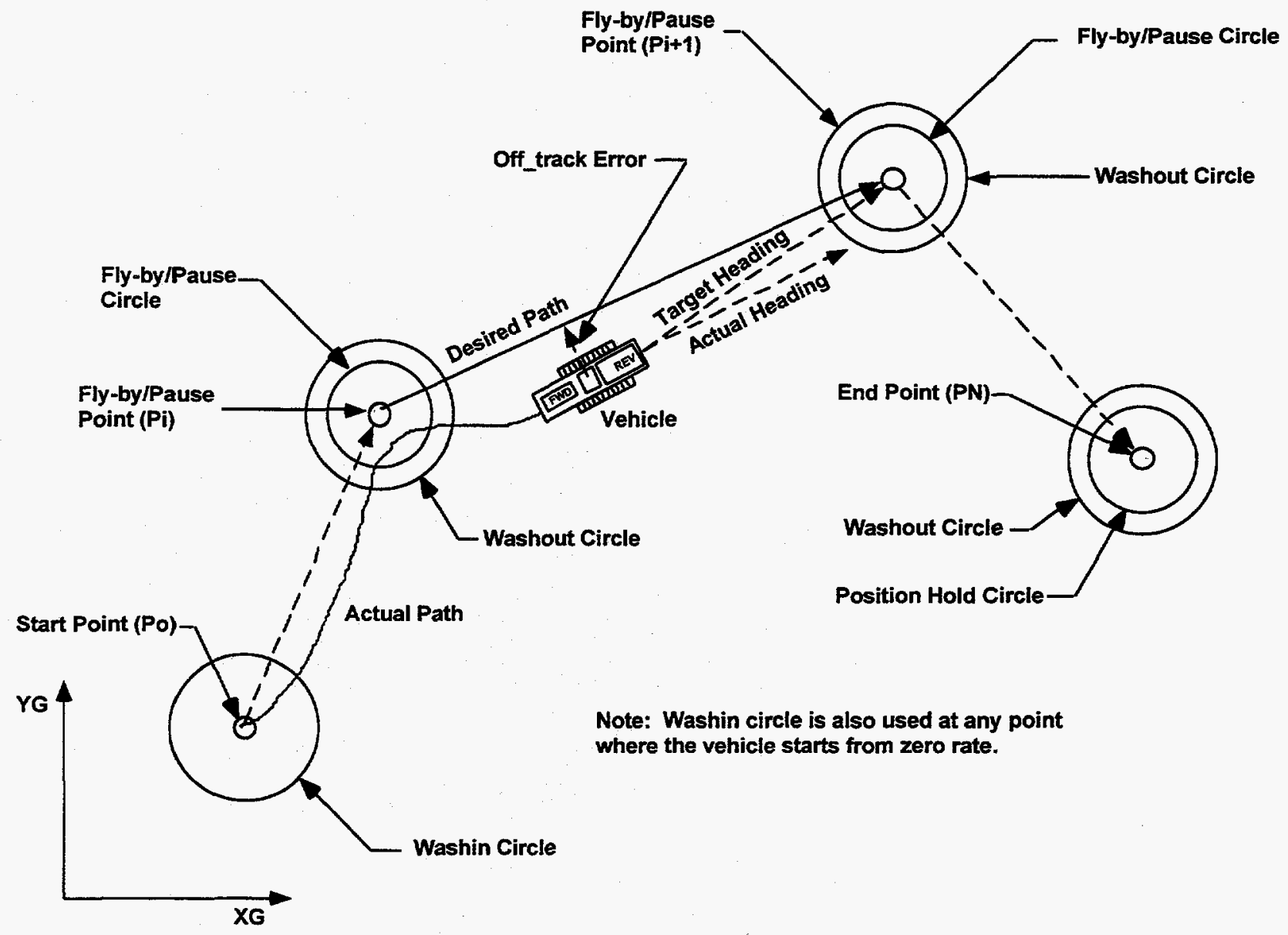

Figure 7. The SGTV path is defined by a series of waypoints that include a start point, fly-by points, pause points, and an end point. 


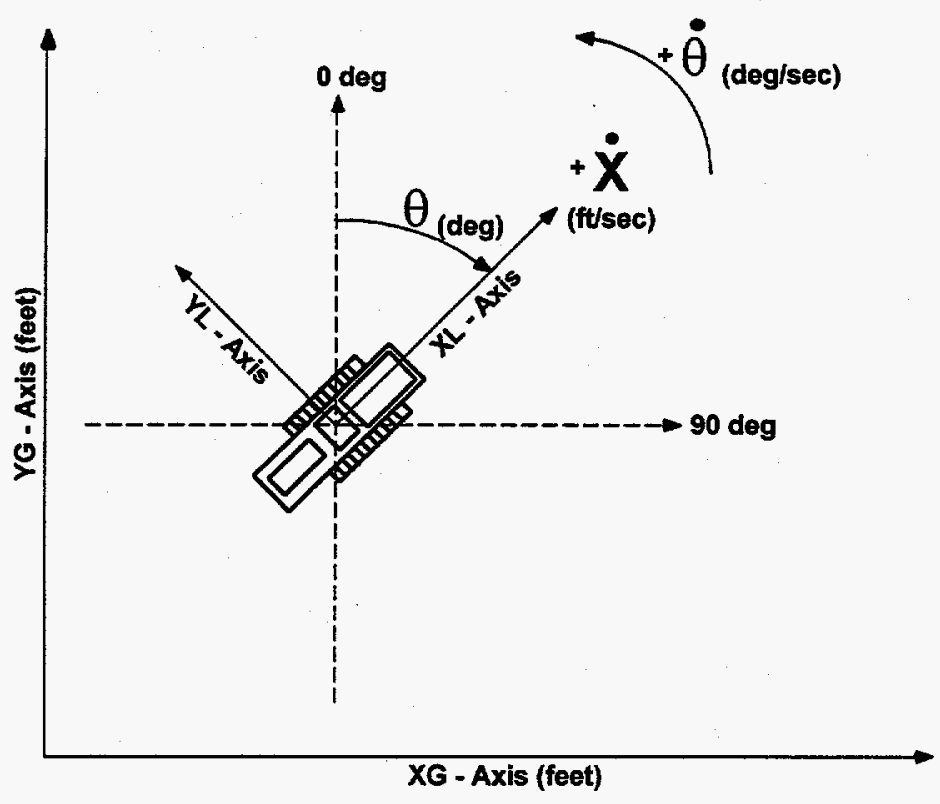

Figure 8. A coordinate system and set of units were established to define the SGTV navigation rules.

The desired rate limits for each path segment can be entered together with the planned path. There is also a global rate limit specified in the control parameter list. The actual rate limit is the minimum of the planned limit and the global limit set for the vehicle.

2.1.6.2.3 No-Go Zone Editor-The No-Go Zone Editor is a windows-based editor with the map of the test site as the background. The program initializes from a file upon execution (i.e., reads a list of configuration items from a file with a predefined name such as rahco.ngz, or inel.ngz). The initialization defaults to a given name, but a pop-up window is displayed to allow the user to select alternate filename(s), and directory names, as well as hard disk name. The initialization file also has a list of parameters needed to satisfy all the configurable items within the application itself.

The no-go zones are defined using a mouse or by entering the $x-y$ location of rectangular zones. In case of a round obstacle, the input parameters are the center of the obstacle and the radius. Once a no-go zone is defined by the mouse, the parameters of the no-go zone are displayed in a window in which the operator may modify the parameters. The no-go zones consist of two shapes: rectangles and circles. The shape can be selected from a pull-down menu item (i.e., shape...). Once a no-go zone has been completed, it is filled with cross-hatched pattern and a distinct light brown color with red edges.

A buffer zone is also displayed around the no-go zones. The buffer size is input from the initializing file and can be modified from a pull-down menu that will display a pop-up dialog to input the new buffer size. Once modified, the display will reflect the new buffer size. The buffer zone is a configuration item (from pull-down menu) and will be saved to the initialization file. If a value is entered as a modified buffer size, a confirmation pop-up dialog will be displayed to confirm the input. 
All no-go zone primitives (rectangles and circles) are then saved to a file with a predefined name. The file format is ASCII with a single line per primitive. A rectangle has four parameters, namely, no-go zone number, the position of the top-left corner, width and height, while a circle has three parameters; no-go zone number, the position of the center, and the radius.

To create the no-go zones, a conversion factor is used to convert $\mathrm{ft}$ to pixels. For example, a $250 \times 250 \mathrm{ft}^{2}$ test site can be converted to a $500 \times 500$ pixel test site (on-screen) with a conversion factor of $6 \mathrm{in} . /$ pixel. Since the graphics resolution of the hardware is $1024 \times 768$, the pixel-to-inch conversion is chosen to maximize windows size, to improve the test site map accuracy.

Primitives (shapes) may overlap to create more complex shapes. Any shape, using the octree method, can be resolved to a series of rectangles of various sizes.

The map and no-go zones are shown in Figures 9 and 10 respectively.

2.1.6.2.4 Waypoint Editor-The Waypoint Editor is used for entering paths as defined in Section 2.1.6.2.2. It is an integral part of the No-Go Zone Editor since they share many functions. Switching between the No-Go Zone Editor and the Waypoint Editor is accomplished through the pull-down menu. Once the no-go zones have been entered, the operator may begin entering the waypoints.

The waypoints are entered using either a mouse or a keyboard. First, a waypoint is entered with a click in the approximate desired location. Once a waypoint is entered, a dialog box is displayed with the position of the point and the default control parameters. There are three control parameters associated with each waypoint; maximum linear and angular rate limit for the next path segment, the type of waypoint ("S" for Start Point, "P" for Pause Point, "F" for Flyby Point and " $E$ " for End Point), and the radius of the Pause Point or Flyby Point. The operator may modify the parameters using the keyboard.

To enter the next point, the operator moves the cursor to the last point and drags it to the next desired position. When the mouse is released, a dialog box will pop up for the operator to modify and confirm.

After the waypoints have been entered, intermediate points may be adjusted. The control parameters associated with each point can also be edited by clicking at that point. A dialog box pops up allowing the parameters to be modified.

The collision detection function can be used to predict potential collisions with no-go zones. If a collision is detected, the waypoints can be modified to avoid collision.

Samples of the Waypoint Editor are shown in Figures 11 and 12.

2.1.6.3 Vehicle Command and Control Generator. The VCCG is a software program for generating the commands to control the vehicle in self-guided control. The basic commands are the linear and angular rate commands for the vehicle to track a path. There are also other commands such as "go," "pause," "resume" and "stop," which can be initiated by the operator or generated by the software based on the control status. For example, if a telemetry error 


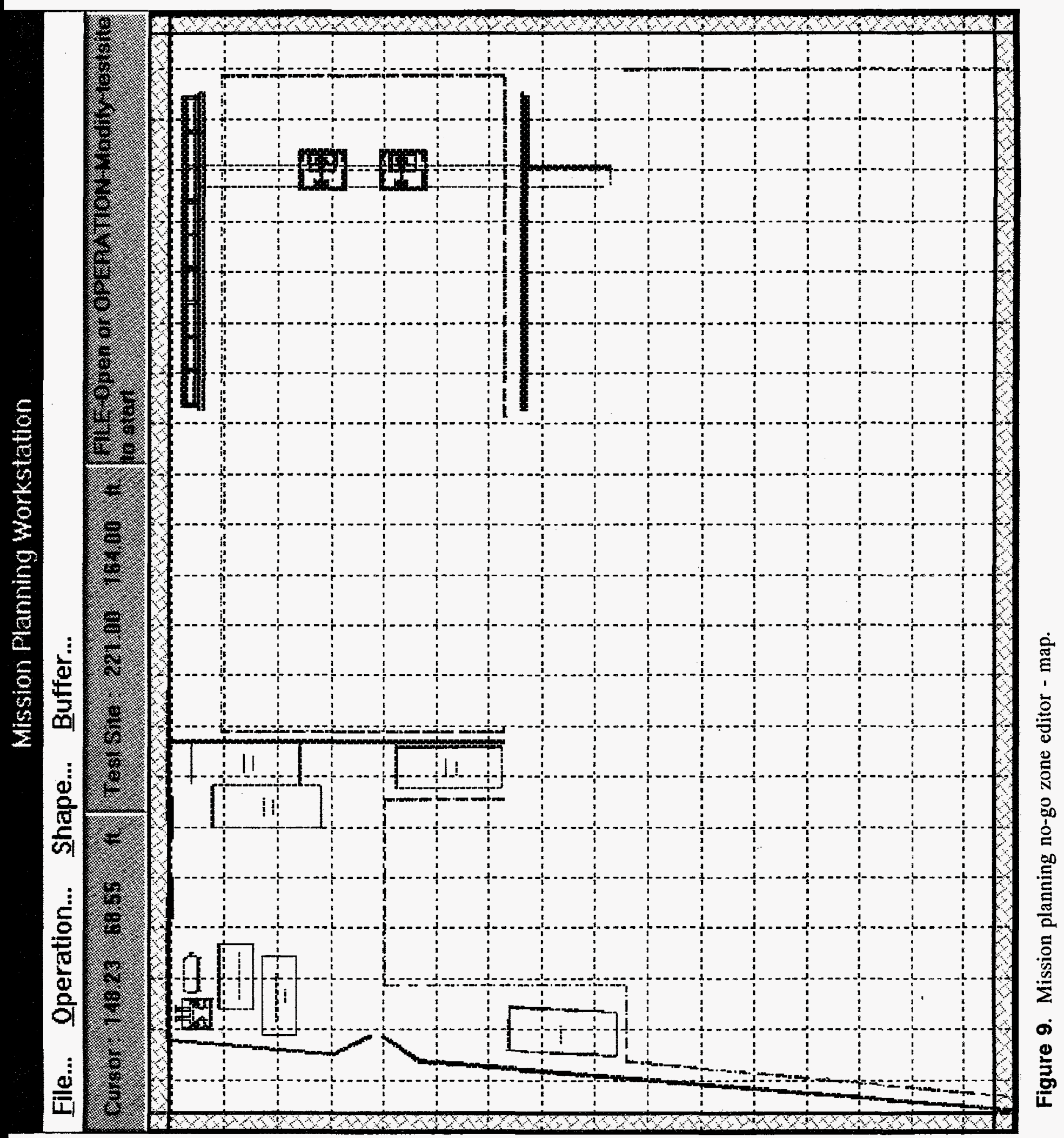




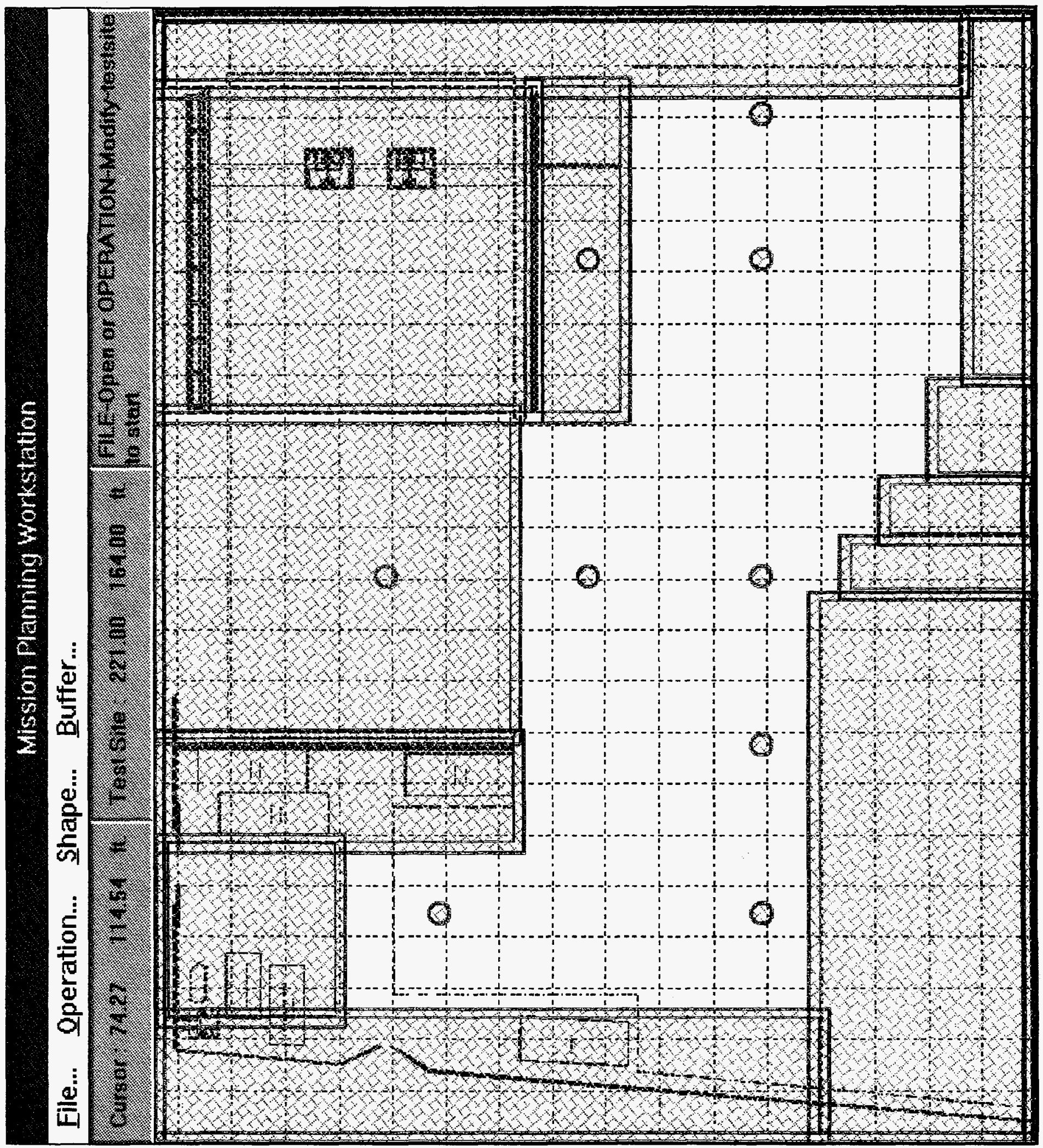




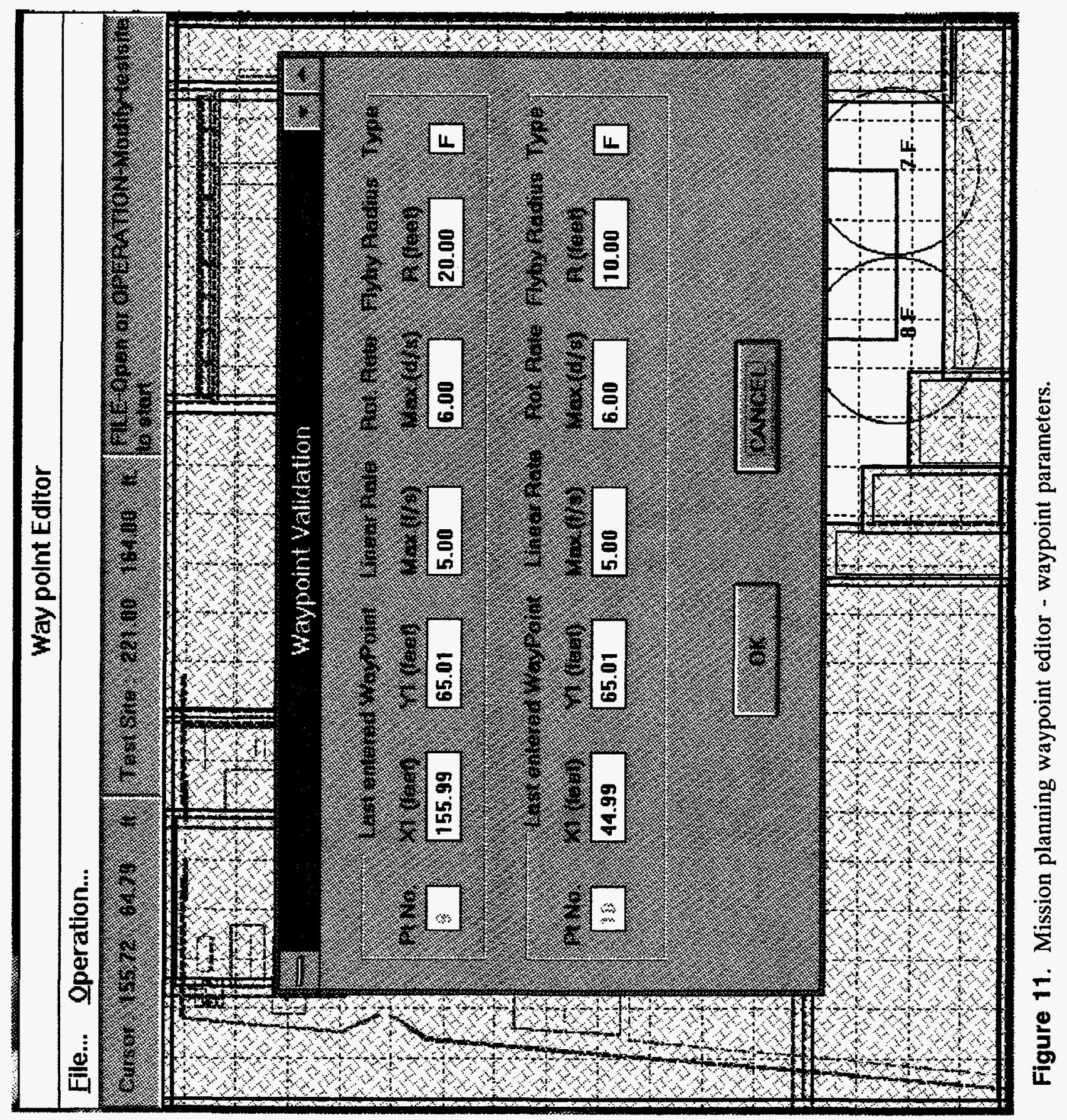




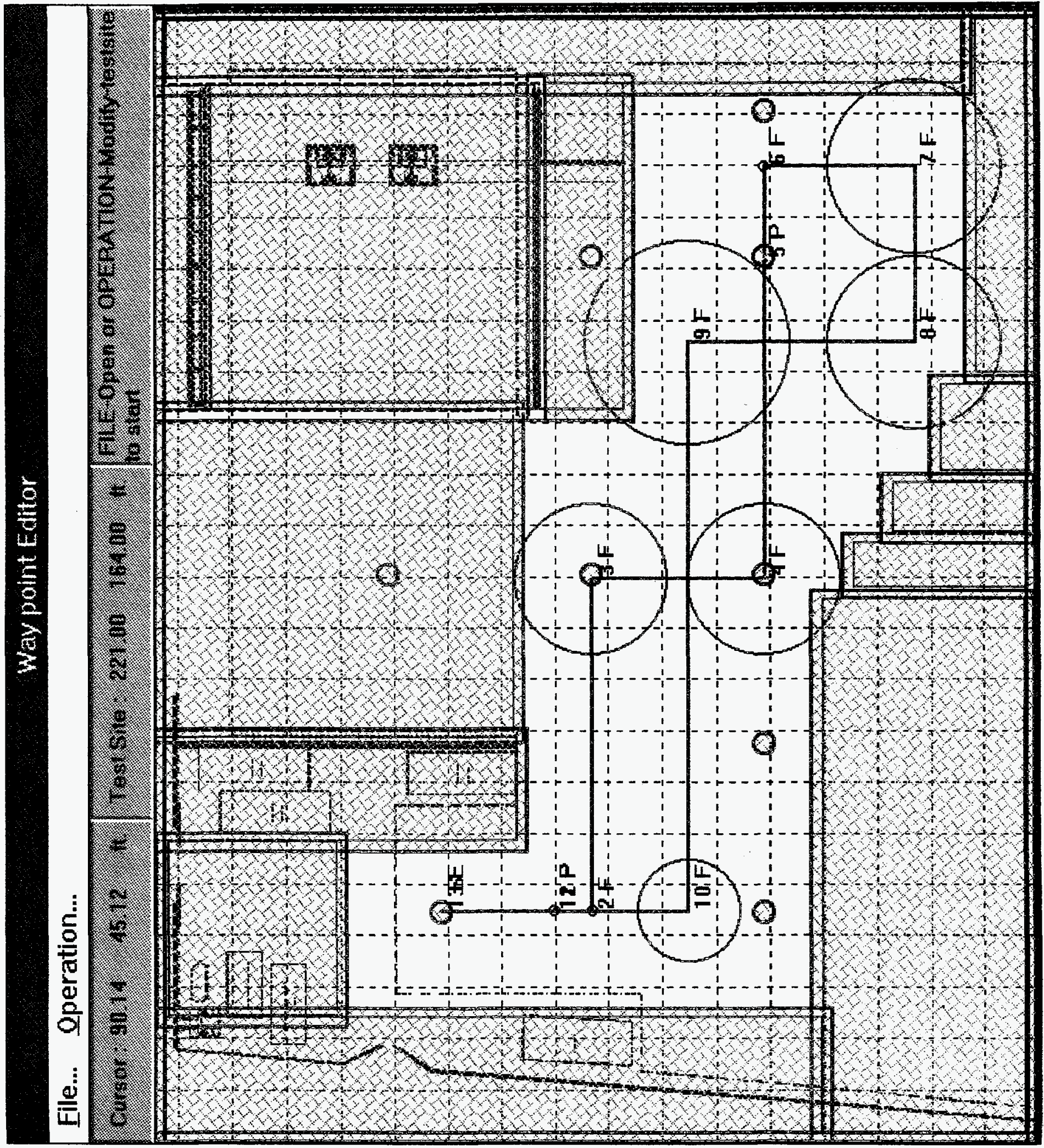


is detected in three consecutive cycles, the vehicle will be paused. After the error has been cleared, the operator may command the vehicle to resume tracking.

A major part of the VCCG is the GUI for the operator to interact with the tracking operation. The functions of the GUI are described in section 2.1.6.3.2.

The core function of the VCCG is to control the off-track error and the heading error of the vehicle relative to the desired path. The details are described in section 2.1.6.3.3.

2.1.6.3.1 Main Features-The VCCG has the following main features:

1. Developed in the cost-effective MS Windows environment using Visual Basic.

2. Has a user friendly GUI for data input, data output, graphical display, data logging, and operator interruption.

3. Has a robust control system to guide the vehicle to track a planned path.

4. Has a kinematic simulation function that allows the operator to analyze the performance of tracking, such as collision avoidance, before running the real vehicle.

2.1.6.3.2 Graphical User Interface-The GUI is shown in Figure 13. It facilitates seven areas of operations as follows:

1. Operator commands

2. Data input

3. Data output

4. Graphical display of tracking

5. Health monitor

6. Data logging

7. Data conversion.

Each area of operation is described in the following:

1. Operator Commands

The operator command buttons reside on the right-hand side of the screen. The first two buttons are "Teleoperated" and "Self-Guided," which are used to select and display (light up) the mode of operation. As a safety feature, the operator must also turn on the self-guided mode of operation through the rotary switch on the control station, which is located next to the VCCG workstation. 


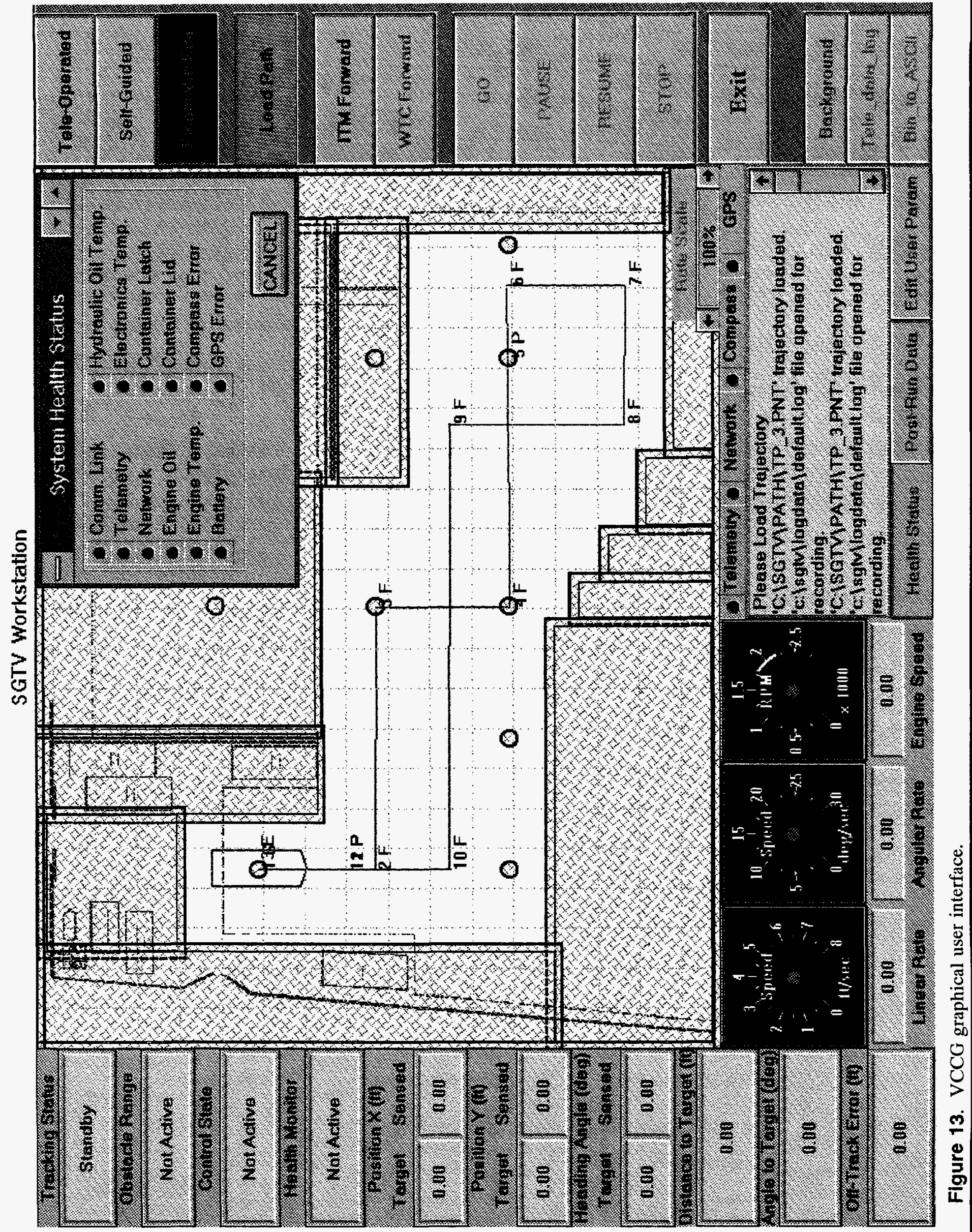


"Kinematic Sim": Allows the operator to run kinematic simulations.

"Load Path": Allows the operator to load a planned path and also name the data logging file.

"ITM Forward": Allows the operator to select the ITM end of the vehicle as the front of the vehicle. In this command, the operator may also assign either end of the planned path to be the start point.

"WTC Forward": Allows the operator to select the WTC end of the vehicle as the front of the vehicle. In this command, the operator may also assign either end of the planned path to be the start point.

"Go": Allows the operator to start tracking. The operator will be prompted to confirm the execution with the Operation Supervisor before accepting the command. The confirmation is not required in a kinematic simulation.

"Pause": Allows the operator to pause the tracking at any time.

"Resume": Allows the operator to resume the tracking after the vehicle has been paused. The operator will be prompted to confirm the execution with the Operation Supervisor before accepting the command. The confirmation is not required in a kinematic simulation.

"Stop": Allows the operator to stop the tracking. The operator will be prompted to confirm the execution. Unlike "Pause," pressing "Stop" allows the operator to load a new planned path or reload the last path.

"Exit": Allows the operator to exit from the VCCG program.

"Background": A allows the operator to select the background for displaying trajectories. Three backgrounds are available: map of the test site with no-go zones (default), map of the test site only, and no-go zones only.

"Tele_data_log": Allows the operator to start or terminate data logging in Teleoperated mode or Direct Drive mode. Teledata log is not needed in the Self-guided mode because data logging is started automatically when tracking begins and terminated when tracking stops.

"Bin_to_ASCII": Allows the operator to convert the binary data file to ASCII format, for post processing.

2. Data input

There are two types of data inputs for the VCCG: planned path data and control parameters. The planned path data is input through the "Load Trajectory" command button. The control parameters are loaded automatically, but there are some parameters which the operator is allowed to modify. The modification is performed in the "Edit User Param" utility. 
3. Data output

There are two types of data outputs: Digital display and pseudo-analog display on the screen. The digital displays are located on the left-hand side of the screen, which include: Tracking Status, Obstacle Range, Control State, Health Monitor, Target Position X \& Y, Sensed Position X \& Y, Target Heading, Sensed Heading, Distance to Target, and off-track Error.

Pseudo-analog (gauge) displays are located at the bottom of the screen, which include Linear Rate, Angular Rate, and Engine Speed. The numerical values of the variables are also displayed at the bottom of the gauges.

4. Graphical display of tracking

The graphical display includes the map of the worksite with grid overlay, nogo zones, vehicle, planned path, and actual vehicle path. All of the information are displayed in real time.

5. Health monitor

The GUI displays the health status of the following elements:

- Communication link between the VCCG computer and the Telemetry \& Control Router

- Telemetry state of the RF link

- Network state at the onboard computers

- $\quad$ Engine oil pressure of the vehicle

- Engine temperature of the vehicle

- Battery level of the vehicle

- Hydraulic oil temperature of the vehicle

- Temperature of the onboard computer subsystem

- Container latch state

- Container lid state

- Compass state

- $\quad$ GPS state.

The status of each element is represented by a red or green light. A green light indicates healthy and a red light indicates an error. The entire health 
monitor windows can be displayed by clicking the "Health Status" button. There are four critical elements: Telemetry state, Network, Compass state and GPS state, which are also displayed permanently on the screen.

6. Data logging

Data logging is performed internally with the file name assigned by the operator. The selected variables to be logged are: time, off-track error, linear command rate, linear rate response, angular command rate and angular rate response. Currently, the data is logged in every cycle and is limited to six variables to reduce computing time. The data are stored in binary format. A utility "Post-Run Data" can be used to plot the data against cycle.

The errors that occur during an operation are also logged. Unlike data logging, error logging has a fixed file name. If necessary, the file can be copied or renamed after an operation. The error messages are stored in a ASCII file which can be reviewed after an operation.

7. Data conversion

Data conversion is a utility to convert the binary file of the logged data to ASCII format. This feature streamlines the post-run study that allows the data to be analyzed using a spreadsheet program such as Excel. To perform data conversion the "Bin_to_ASCI" button is depressed and the operator is asked to specify the file name.

2.1.6.3.3 Self-guided Control-The next two subsections describe the control approach and the architecture of the self-guided control, as well as the principal algorithms.

\section{A. Control Approach}

The vehicle has two degrees of freedom: a linear motion and a rotation about the center of the vehicle. The control approach is to use the linear rate to control the vehicle to reach a target point, while the angular rate is used to correct the off-track error and heading error. The magnitudes of the linear rate commands are scaled to reduce the overshoots during startup and stopping.

The path tracking approach is as follows: The SGTV onboard computer estimates the current position and heading of the vehicle based on the GPS data and the Dead Reckoning estimates. It then sends them to the MP \& VCCG computer, which compares the current position and heading with the target point and the desired heading to generate the position and heading errors. The magnitude of the linear rate command is determined according to the position error. The angular rate command is computed to correct the heading and off-track errors.

The operation of the self-guided control is as follows:

The first step is to select the self-guided mode through the rotary switch on the Control Station, then load the planned path. The next step is to command the vehicle to move to the start point of the planned path. This may be done manually in Teleoperated mode, in Direct Drive 
mode, or automatically in self-guided mode. If the self-guided mode is used, the operator is responsible for ensuring that the path between the current position of the vehicle and the start point of the planned path is clear.

After the vehicle has reached the start point, it will start moving to the next target point, which may be a "flyby" point, "pause" point or the "end" point. When the vehicle enters the "flyby circle" of a "flyby" point, it will start moving to the next target point immediately. There are two types of "flyby" points. First, if the radius of the flyby circle is not greater than two $\mathrm{ft}$, it is a regular flyby point. In this case, the vehicle will correct the heading error first before it starts moving to the next target point. Second, if the radius of the flyby circle is greater than $2 \mathrm{ft}$, it is called a smooth flyby point. In this case, the vehicle will move from the current path segment to the next path segment with smooth turning; that is, it corrects the off-track error and heading error simultaneously.

When the vehicle enters the "pause circle," it will be stopped by setting the commanded rates to zero. When the "resume" command is activated by the operator, the next target point is loaded and executed. At any time during the self-guided control, the operator may also pause the motion. In this event the vehicle is stopped immediately by setting the rate commands to zero. The operator may then activate the "resume" command to continue tracking.

Whenever the vehicle is started from zero rate, the linear rate command will be ramped up gradually with the ramp up speed derived from the "washin radius."

When the vehicle enters the "washout circle," the rate commands will be reduced gradually until it reaches the target circle, which may be a "flyby circle," "pause circle" or "position hold circle." If the target circle is a flyby, the next target point will be loaded and the vehicle control will continue. If the target point is a pause, the vehicle will stop and wait for the next command from the operator. The next command may be resume or stop. If the target point is the final point, the vehicle will stop and the self-guided control is completed.

When the vehicle pauses or reaches the target point, the operator may command it to reverse the path with either end of the vehicle as the front.

During the operation, errors (health status) may occur. In such case, the operator will be informed of the error so than he can determine whether to stop the operation or not. However, telemetry errors, network errors and GPS errors, may cause the vehicle to lose control.

Therefore, the vehicle is paused automatically when such errors occur. Note that this feature was disabled during the Factory Acceptance Test in order to evaluate the impact of the errors on the control performance.

\section{B. Control Functions}

The block diagram of the VCCG self-guided control module is shown in Figure 14. It consists of the following functions:

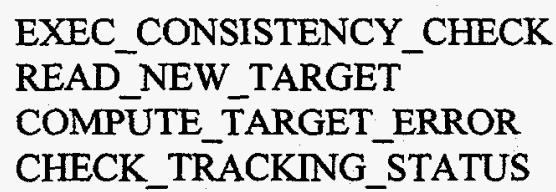




\section{EXEC_PATH_TRACKING \\ RATE_CMD_PROCESSOR.}

Each function and its algorithms will be described in the following subsections. Note that the control algorithms use $\mathrm{ft}, \mathrm{ft} / \mathrm{sec}$, radians and radians/sec as the units. Appropriate unit conversions on input and output data are necessary.

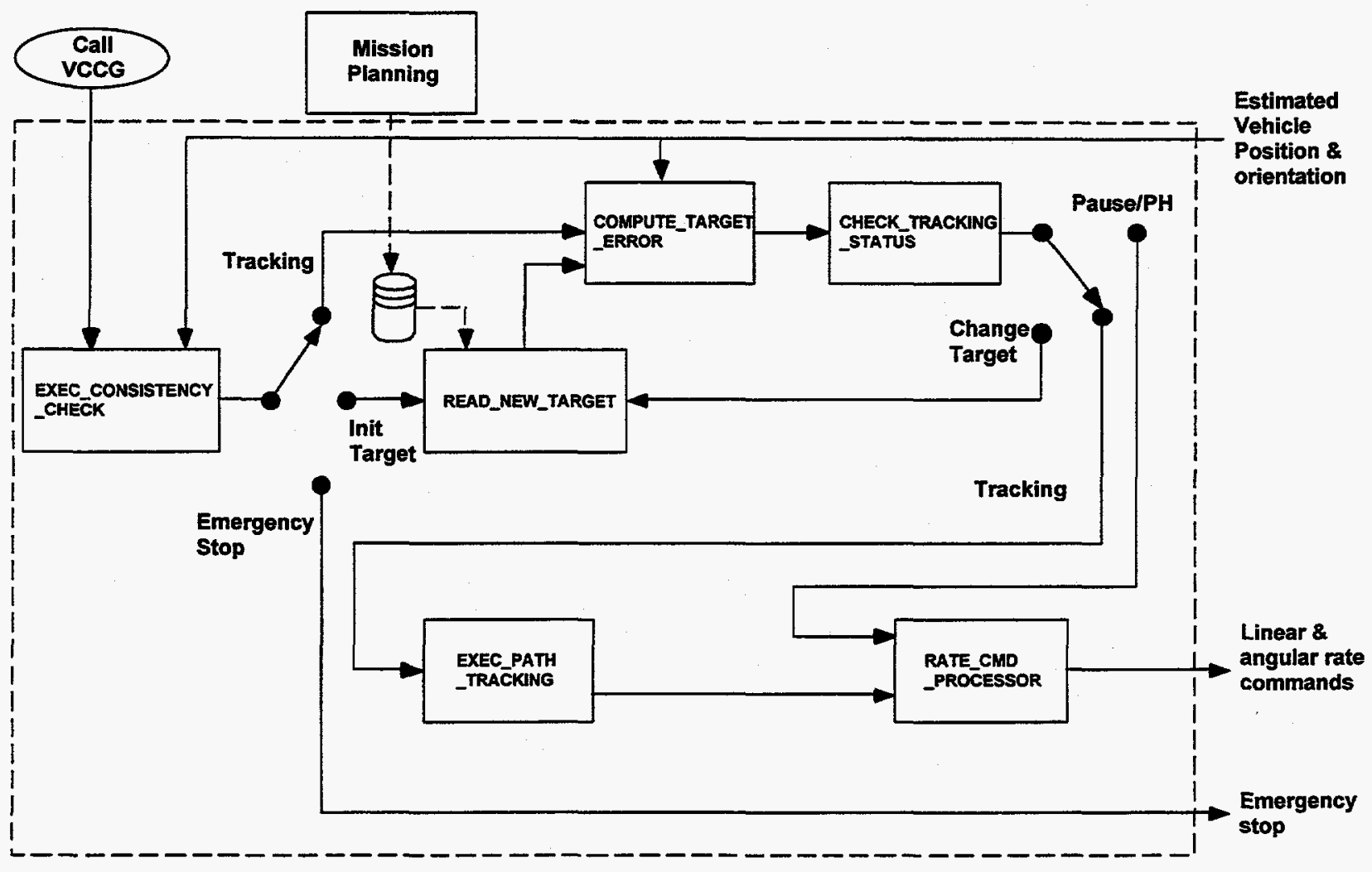

Figure 14. The VCCG self-guided control module functional organization.

- $\quad$ EXEC_CONSISTENCY_CHECK. This function monitors the off-track error and the position error. If an error exceeds a predefined limit, a failure occurs and the VCCG will set the rate commands to zero and issue an emergency stop command to the onboard computer. The operator is also notified of the failure by the VCCG

- READ_NEW_TARGET. This function reads a target from a planned path for the VCCG to execute. Based on the operator's selection, it can also reverse the planned path; that is, it can use the end point as the start point and vice versa. After the vehicle reaches a flyby circle, or a pause circle, or is paused by the operator, it can determine the next target point. 
For "Forward Tracking," the control algorithm uses the first point of the planned path as the start point and the last point as the end point. For "Reverse Tracking," the sequence is reversed.

- COMPUTE_TARGET_ERROR. This function computes the norm of the position errors, heading error and off-track error. The norm of the position errors is calculated as follows:

In both "Forward Tracking" and "Reverse Tracking," the feedback positions are:

Pos_Fbk_X $=$ Measured_Pos_X

Pos_Fbk_Y $=$ Measured_Pos_Y

The position errors and norm are:

$$
\begin{aligned}
& \text { Pos_Error_X }=\text { Target_Pos_X -Pos_Fbk_X } \\
& \text { Pos_Error_Y }=\text { Target_Pos_Y }- \text { Pos_Fbk_Y } \\
& \text { Pos_Norm }=\text { sqrt(Pos_Error_X^2 }+ \text { Pos_Error_Y^2) }
\end{aligned}
$$

The heading error is calculated as follows:

In "Forward Tracking", the heading angle feedback is:

$$
\text { Heading_Ang_Fbk }=\text { Measured_Heading_Ang }
$$

In "Reverse Tracking", the heading angle feedback is:

$$
\text { Heading_Ang_Fbk }=\text { Measure_Heading_Ang }+ \text { pi }(=1800)
$$

The target heading is:

$$
\text { Target_Heading_Ang }=\tan -1 \text { (Pos_Error_Y/Pos_Error_X) }
$$

When the vehicle is near the target point, the Target_Heading_Ang is changed to:

$$
\begin{aligned}
& \text { Pos_C_X }=\text { Target_Pos_X }- \text { Init_Pos_X } \\
& \text { Pos_C_Y }=\text { Target_Pos_Y }- \text { Init_Pos_Y } \\
& \text { Target_Heading_Ang }=\text { tan-1(Pos_C_Y/Pos_C_X) } \\
& \text { Heading_Ang_Error }=\text { Target_Heading_Ang }- \text { Heading_Ang_Fbk }
\end{aligned}
$$

The off-track error is:

$$
\text { Pos_C_Norm }=\operatorname{sqrt}\left(P o s \_C \_X^{\wedge} 2+\right.\text { Pos_C_Y^2) }
$$




$$
\begin{aligned}
& \text { Pos_T_X }=\text { Measured_Pos_Y - Init_Pos_X } \\
& \text { Pos_T_Y }=\text { Measured_Pos_Y - Init_Pos_Y } \\
& \text { Off_Track_Error }=\left(\text { Pos_T_X }{ }^{*} \text { Pos_C_Y }- \text { Pos_T_Y } * \text { Pos_C_X }\right) / \text { Pos_C_Norm }
\end{aligned}
$$

- CHECK_TRACKING_STATUS. This function uses Pos_Norm to determine the tracking status. The conditions for determining the tracking status and rate command scales are:

Initial Condition: During the period when the planned path is set up and the controller parameters are updated.

Lin_Rate_Cmd_Scale $=0.0$

Washin Condition: Washin_Factor $<1.0$

where

Washin_Factor $=($ Washin_Time/Washin_Completion_Time $)$

Washin_Exp_factor

and

Washin_Completion_Time $=$ Pos_Washin_Radius/Linear_Vel_Limit

Lin_Rate_Cmd_Scale $=$ Washin_Factor, if Washin_Factor $<=1$

Lin_Rate_Cmd_Scale $=1$, if Washin_Factor $>1$

Washout Condition: Pos_Norm $<$ Pos_Washout_Radius

Washout_Factor $=$ Pos_Norm/Pos_Washout_Radius

Lin_Rate_Cmd_Scale $=$ Washout_Factor

Flyby Condition: Waypoint_Type $=$ "F", and Pos_Norm $<$ Pos_Flyby_Radius

and Pos_Flyby_Radius $<=2 \mathrm{ft}$

Lin_Rate_Cmd_Scale $=0$

Smooth Flyby Condition: Waypoint_Type $=$ "F", and Pos_Norm $<$ Pos_Flyby_Radius

and Pos_Flyby_Radius $>2 \mathrm{ft}$ 
Pause Condition: Waypoint_Type $=$ "P", and Pos_Norm $<$ Pos_Pause_Raảius

Lin_Rate_Cmd_Scale $=0$

Position Hold Condition: Waypoint_Type $=$ "E" and Pos_Norm $<$ Pos_Hold_Radius

$$
\text { Lin_Rate_Cmd_Scale }=0
$$

Between Washin \& Washout Condition: Satisfy Washin and Washout conditions

Lin_Rate_Cmd_Scale $=$ Min(Washin_Factor, Washout_factor)

Max. Rate Condition: None of the above and the vehicle is between washin circle and washout circle.

$$
\text { Lin_Rate_Cmd_Scale }=1
$$

- EXEC_PATH_TRACKING. Linear rate command is determined by the Lin_Rate_Cmd_Scale, which is associated with tracking status, as follows:

$$
\text { Lin_Vel_C }=\text { Lin_Rate_Cmd_Scale }{ }^{*} \text { Lin_Vel_Limit }
$$

where

Lin_Vel_Limit is the selected linear velocity command limit.

Angular rate command is determined by the heading angle error and off-track error as follows:

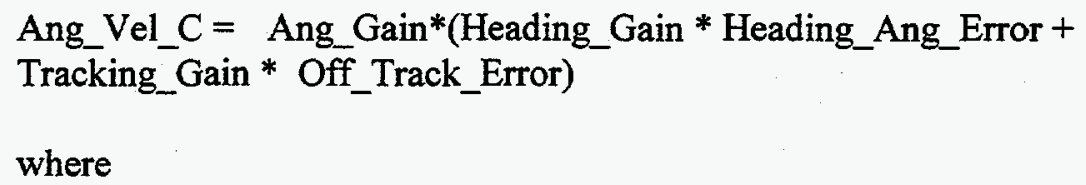

where

Heading_Gain: Heading controller gain

Tracking_Gain: Off-track controller gain

Ang_Gain: Overall control gain

To ensure the vehicle does not drive in the opposite direction when the off-track error is much larger than the heading error, the following method is used:

if $\mid$ Tracking_Gain * Off_Track_Error $|>|$ Heading_Gain * Heading_Ang_Error $\mid$ then

Tracking_Gain $=0.9 * \mid$ Heading_Gain $*$ Heading_Ang_Error/Off_Track_Error $\mid$ 
Another effective ad hoc method to reduce the off-track error is: When the heading error is less than a preset value, say 5 degrees, the linear and angular controllers are both executed together. When the heading error is greater than the preset value, the linear rate command is set to zero temporarily until the heading error falls within the preset value. This may occur when the vehicle begins to track a new target point.

The smooth flyby control also uses the same type of algorithm but with slightly different gains.

- RATE_CMD_PROCESSOR. This function limits the rate commands not to exceed the selected rate limits. It also sets the rate commands to zero for Pause, Stop, Flyby, and Position Hold.

The direction of the linear rate command is determined by whether the vehicle is in ITM Forward or WTC Forward. For ITM Forward, Lin_Vel_C is multiplied by Lv_Dir $=1$, whereas for WTC Forward Lv_Dir $=-1$.

\subsubsection{Retrieval Support Equipment}

The Retrieval Support equipment consists of the cooperative Retrieval Hoist-ITM Adapter and the ITM/WTC Handling Device.

2.1.7.1 Cooperative Retrieval Hoist-ITM Adapter. The hoist-ITM adapter, Figure 15, consists of the following: a lift point; a guide skirt; latch mechanisms; electrical/hydraulic quick disconnect manifold; and the necessary actuating hydraulic cylinders.

The lift point is a steel bar that is welded to the hoist-ITM adapter and is used to integrate the 5 ton crane hoist to the ITM adapter.

The hoist-ITM adapter has a flared guide skirt with a 3-in. draft to accommodate easy attachment of an empty or full ITM to the crane hoist. A latch mechanism on each side of the hoist-ITM adapter assembly secures the ITM to the assembly. Each latch is equipped with two rotating dogs that lock the ITM to the hoist-ITM adapter assembly. This provides a positive, failsafe connection. 


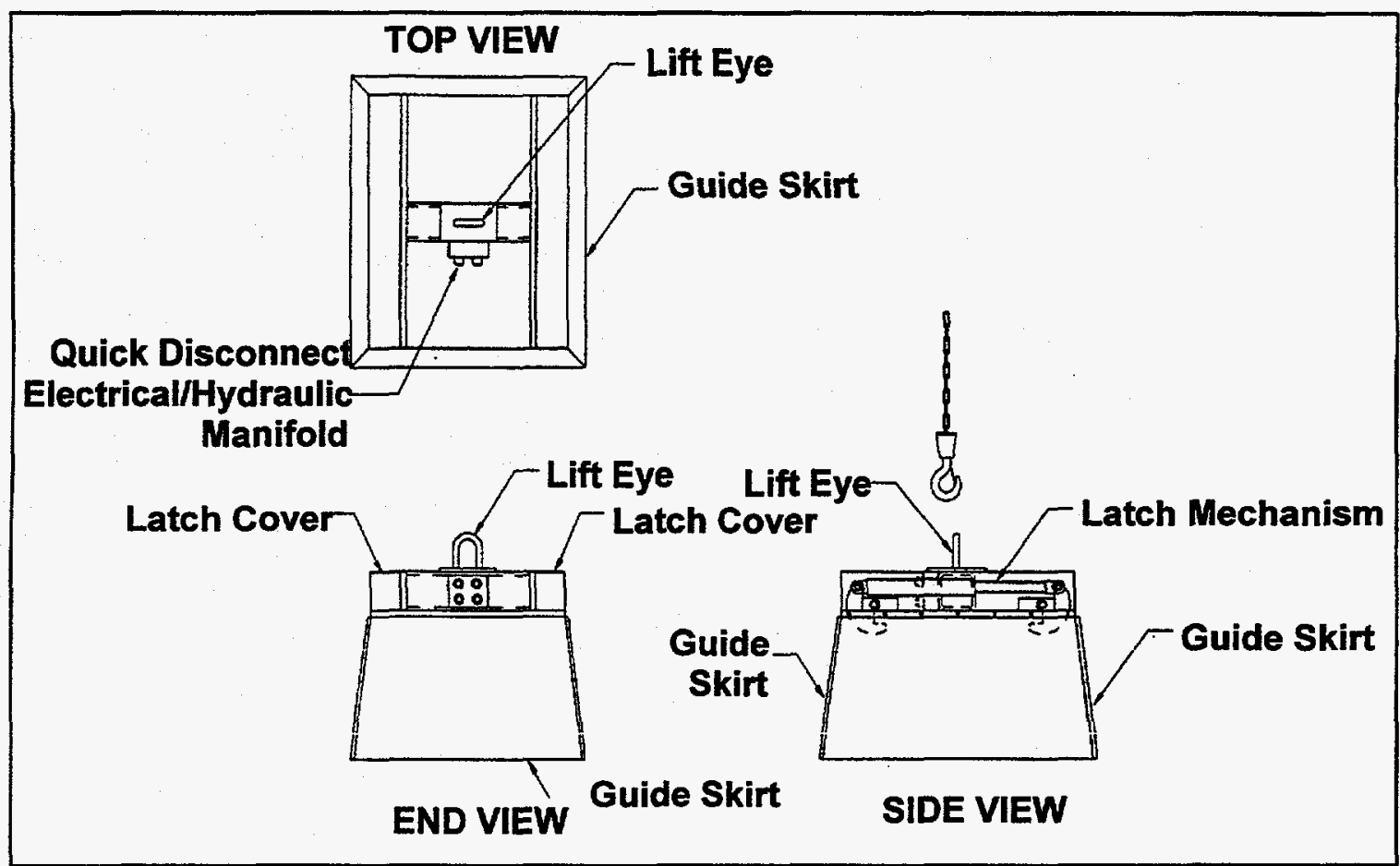

Figure 15. The Cooperative Retrieval ITM Hoist Adapter provides a method for the gantry crane to manipulate ITMs. The adapter shares many of design features with the Innovative End Effector.

An electrical/hydraulic quick disconnect manifold is provided to allow single-point contact for the electrical and hydraulic connections. This enables the electrical and hydraulic connections to be made telerobotically.

2.1.7.2 ITMMTC Handling Device. The ITM is configured with lift pockets to facilitate handling and dumping with a standard forklift and forklift attachment. A forklift attachment was provided that enables the forklift to rotate the ITM 180 degrees for dumping. 


\section{TEST OBJECTIVES AND EXECUTION}

The overall objective of the demonstration was to verify that the SGTV operates as designed and to gather data to document its performance characteristics. Data was obtained with the best available instrumentation and techniques within the schedule and budget of the project. All instrumentation was operating within the stated calibration range, with total uncertainty as stated herein.

\subsection{Test Objectives and Data Quality Objectives}

The following discussion of the test objectives includes a statement of the test objective, the data quality objective (DQO) for that test objective, and a statement of the uncertainty for the measurements to be taken to meet the $\mathrm{DQO}$, with a short discussion of how each uncertainty specification was derived.

\subsubsection{Operational and Transuranic Environment Evaluation}

Test Objective: Evaluate the capability of the system to operate in a transuranic environment.

Data Quality Objective: Observe SGTV operations and record information concerning dust generation (qualitative visual description), reliability, ease of maintenance, and any other operational parameters that could be used to determine the system's capability to operate in a transuranic retrieval environment. Documentation shall include photographs, videotape, and logbook entries. Specific items to document include:

- Visual observations of dust generated during operations

- Anomalous conditions

- Overall reliability of SGTV

- Frequency and type of maintenance required during the demonstration, both scheduled and unscheduled

- Ruggedness of the equipment

- Length of time required for maintenance activities

- Ability of the system to easily correct for control system errors and/or operator errors.

Uncertainty: No uncertainty specifications for qualitative data.

\subsubsection{Throughput}

Test Objective: Measure the production rate for transportation of waste from the digface based on use of the selected equipment and technologies. A goal of $80 \mathrm{yd}^{3} /$ day in an 8 -hour shift will be used for design of the demonstration system. 
Data Quality Objective: Document the cycle times for the SGTV by measuring the time for the SGTV to travel from the digface to the unloading station, including time for loading/unloading procedures (opening/closing lids, etc.).

Uncertainty: The following total uncertainty specifications apply for the measurements:

- The time required: 0.25 minute per cycle.

The 0.25 minute total uncertainty was derived using engineering judgment. This time accuracy was selected because it is easy to measure and provides sufficient accuracy to satisfy the DQO.

\subsubsection{Miscellaneous Human Interface, Safety, and Vehicle/Control System Performance Parameters}

Test Objective: Demonstrate/document both the self-guided and telerobotic control system human interface, safety of operations, and vehicle performance parameters from measurements, observations, and operator interviews taken during the demonstrations.

Data Quality Objective: Document the operations of the remote control system and the ability of the system to effectively control the remote transportation system using photographs, videotape, logbook entries, and operator interview records.

Some data are qualitative by nature, but crucial to understanding the effectiveness of the remote control system. In particular, the human machine interface (human factors) design of the operator control station will be documented.

\section{Human Factors}

Each operator will be interviewed to determine his/her opinion on the following:

- Are the controls located in a logical manner, intuitive to use, and command the SGTV as indicated or expected

- Are the status indicators (lights, LCD display, and audio signal) easily understood and are warning indicators noticeable

- Are the lid and latch control switches easily within reach and adequate for the purpose

- Is the view provided by the SGTV on-board and off-board cameras adequate for the purpose of teleoperation and monitoring in self-guided mode

- Does eye strain result from continuous watching of the video monitor.

\section{Misc. Vehicle/Control System Performance}

- Travel capability: Verify maximum speed of the vehicle by time-to-distance measurements. 
- System health monitoring.

\section{Safety}

Because the operational safety of the SGTV is of the utmost importance, the following will be documented:

- Test and document the function of the hydraulics enable/disable and emergency stop systems

- Anomalies such as the number of times the transportation system responds differently than expected to the sent signal, the number of times the remote transportation system receives an unintended signal, and/or the ability of the control system to regain control if such a malfunction occurs

- Any other system malfunctions

- Safeguards designed to prevent human error

- Operator errors, the response of the system to such errors, and the procedures necessary to correct them.

\section{Mission Path Planning/Vehicle Command Control Generator Performance}

- No-go zone creation

- Path creation

- Display and command graphical user interface (GUI)

- Communications and data logging.

Uncertainty: Not applicable or will be reported with the data.

\subsubsection{System Integration}

Test Objective: Demonstrate the integration between the SGTV and the CTR using the CTR to load and unload ITMs from the SGTV.

Data Quality Objective: Document the level of integration of the SGTV and CTR system and the affect that has on the smooth operation of the system using photographs, videotape, and logbook entries. Specific items to document include:

- How well the SGTV receives and deploys ITMs with the CTR

- To what extent system integration (i.e., fit and smooth operation) was built into the design of the two systems. (That is, are the designs concurrent and integral to each other so that overall system performance is improved). 
Uncertainty: Not applicable.

\subsubsection{Precision and Accuracy of Navigation}

Test Objective: Demonstrate that the SGTV can follow a preplanned path of travel and determine the precision and accuracy of the Differential Global Positioning System/Dead Reckoning (DGPS/DR) navigation system. The demonstration includes setting the SGTV on self-guided mode and measuring the variance between the actual vehicle path and the planned vehicle path.

Data Quality Objective: Document the capabilities of the DGPS/DR system and determine the system's ability to effectively navigate through a predetermined test path. Determine with a $95 \%$ confidence level that for any target path specified, the variation from that target path is no greater than 12 in.

Uncertainty: The following total uncertainty specifications apply for the measurements:

- Variation from planned path:

$$
\begin{aligned}
& \text { - } \quad X \text { position - } 2 \text { in. } \\
& \text { - } \quad Y \text { position - } 2 \text { in. }
\end{aligned}
$$

Heading (clockwise from North) - 0.2 deg (based on performance of the electronic compass)

For the $\mathrm{X}$ and $\mathrm{Y}$ position, the uncertainty was derived from specifications for the accuracy of the Trimble $4000 \mathrm{SSI}$ hardware. For the heading, the 0.2 degree uncertainty was derived from the electronic compass specifications with some conservatism for compass fluctuations noted during development. Manual measurements to fixed points of reference shall be taken with a tape measure at the end-points of the path and any pause points placed in the path to verify the data coming back to the Vehicle Command Control Generator (VCCG). Variation from the planned path will determined from the feedback data to the VCCG and not verified through an independent means. Comparison of end-point and waypoint measurements to VCCG data will be sufficient to verify that data from the DGPS/DR system is accurate.

\subsubsection{SGTV Ultrasonic Obstacle Avoidance}

Test Objective: Demonstrate that the SGTV ultrasonic obstacle avoidance system functions according to specification.

Data Quality Objective: Document the capabilities of the SGTV ultrasonic obstacle avoidance system and determine the system's ability to effectively detect and stop the vehicle before a collision.

Uncertainty: The following total uncertainty specifications apply for the measurements:

- Distance to the obstacle detection: $2.5 \mathrm{ft}$

- Distance to the obstacle when it first enters collision zone: $2.5 \mathrm{ft}$ 
- Distance to obstacle after emergency stop has been initiated by the obstacle avoidance system: 6 in.

The total uncertainty was derived from the specification accuracy and performance of the ultrasonic sensors and/or the use of a tape measure for manual measurements. The uncertainty specified will provide sufficient accuracy to fulfill the objective of the test while accounting for slight misalignment of a tape measure or the specification tolerance of the ultrasonic sensors. Data from the ultrasonic sensors and a manual tape measure will be used to verify distance to target and confirm proper readings from the ultrasonic sensors.

\subsubsection{Maneuverability}

Test Objective: Demonstrate that the operating envelope and maneuverability of the SGTV is within design parameters.

Data Quality Objective: Document the maneuverability and operating envelope of the SGTV with qualitative analyses including, but not limited to, how well the vehicle maneuvers on varying surfaces, different soil conditions, and when traversing a slope. Document the maneuverability and operating envelope with quantitative analysis including, but not limited to, turning radius, turning rate (maximum and minimum practical), close approach envelope to right-angle walls, and obstacle (slalom) course.

Uncertainty: The following uncertainty specifications apply for the measurements:

- Turning radius (true and swing area): 6 in.

- Close approach into 90-degree angled wall: 6 in.

The uncertainty will provide sufficient accuracy to fulfill the objective of the test while accounting for variations in the measurement techniques and equipment. Uncertainty specifications for qualitative data are not applicable.

\subsection{Experimental Design and Procedures}

This section outlines the methods and procedures used to evaluate the SGTV.

\subsubsection{Test Design and Methodology}

The testing was conducted at the INEEL in Idaho Falls, Idaho. A general layout of the INEEL test venue is shown in Figure 16. A test plan entitled "Acceptance/Performance Test Plan for the Self-Guided Transport Vehicle (SGTV)" was prepared and executed by the INEEL Principal Investigator and subcontractor personnel.

3.2.1.1 Operational and Transuranic Environment Evaluation. Operational and transuranic evaluations consisted mainly of qualitative data. This data involved visual dust generation evaluation, anomalous conditions, maintenance requirements, repairability, operational costs, and the overall reliability of the SGTV. Maintenance, repairability, and reliability evaluations were performed through monitoring the frequency, type, and the 


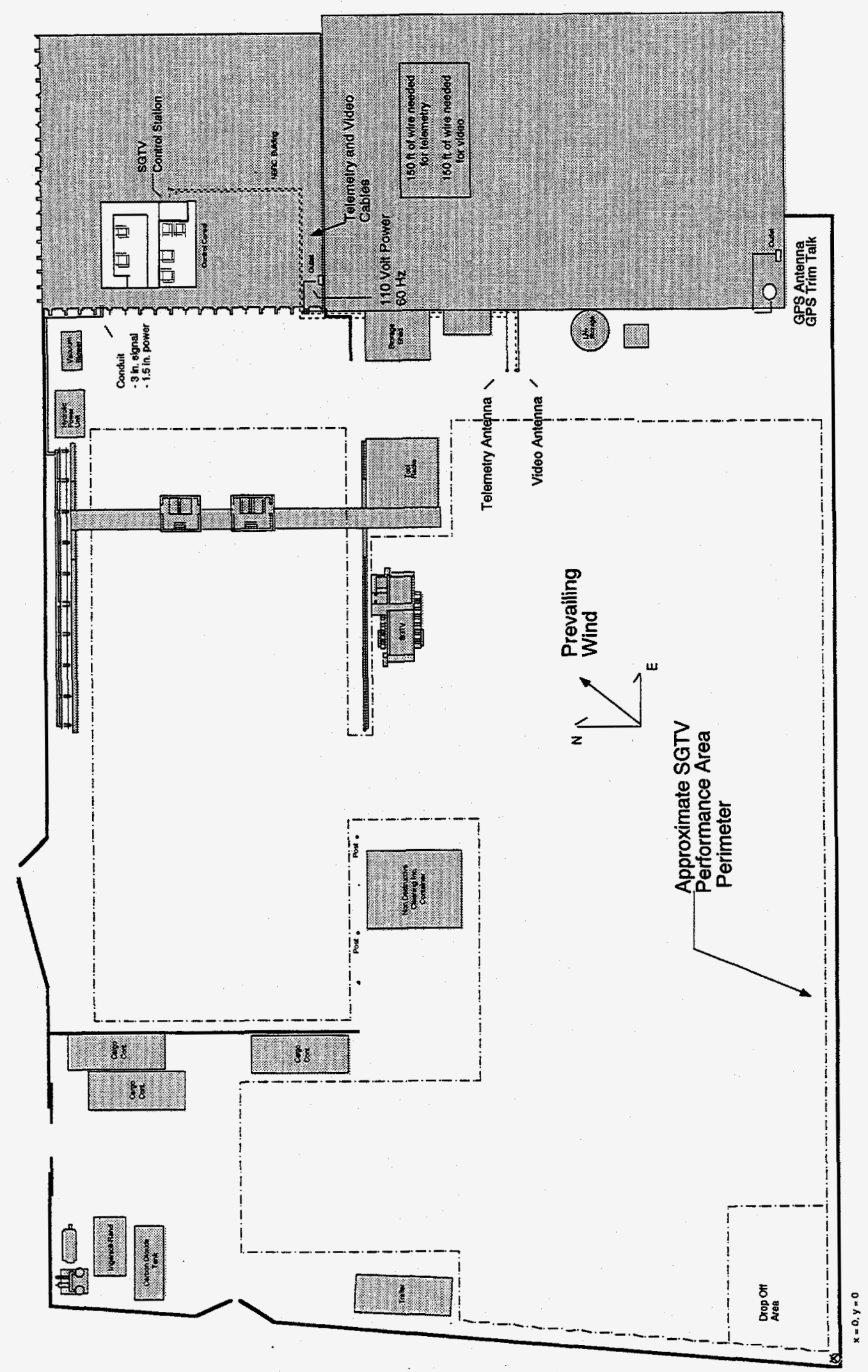

Figure 16. General arrangement of the Hot Spot Retrieval and Transportation Demonstration test area at the INEEL Robotics Laboratory (NBA building). 
approximate length of time for any required maintenance. Operational costs can later be extrapolated using some of this data. Data was documented throughout testing in logbook entries, and where appropriate with photographs and video.

3.2.1.2 Throughput Testing. The maximum throughput of the SGTV will be determined by following the detailed procedure specified in the test plan. Time was recorded between the time the ITMs were to be placed into the SGTV until the ITMs were to be unloaded from the SGTV. Loading and unloading procedures were to be included in the times recorded. Track distance was recorded along with the complexities involved with the vehicle's path. The measurement of time to distance allowed for calculation of the theoretical throughput of the SGTV. Operating conditions such as the terrain, surface conditions, and weather were noted. These time/motion studies were conducted in both telerobotic and self-guided control modes for comparison.

\subsubsection{Miscellaneous HMI, Safety, and Vehicle and Control Performance}

Parameters. Parameters involving safety, human interface, and performance were determined through testing as specified in the test plan. These parameters contain both qualitative and quantitative data.

3.2.1.4 System Integration. System integration was determined through testing and documenting how cohesive separate components are. The testing involved integration between the SGTV, the REMEX with innovative end effector, and the CTR gantry crane. Quantitative data, such as the time of transferring the ITMs, was to be monitored along with qualitative data such as the ease of integration.

3.2.1.5 Precision and Accuracy of Navigation. The GPS/DR navigation system is responsible for precise and accurate navigation. Quantitative data throughout all testing procedures was gathered to determine the effectiveness of the GPS/DR system. The data was obtained primarily from the GPS system and the precision and accuracy determined by calculating the difference between the actual location of the vehicle and expected position of the vehicle (off-track error).

3.2.1.6 SGTV UItrasonic Obstacle Avoidance. The ultrasonic obstacle avoidance system was tested by quantitative means that compare manual measurements with the on-board avoidance system ultrasonic sensors, and in some cases also using data from the GPS system. Measurements taken included the distance an obstacle was first detected, the distance an obstacle first entered the collision zone, and the distance when the emergency stop was initiated by the obstacle avoidance system.

3.2.1.7 Maneuverability. Maneuverability of the SGTV was to be determined through qualitative tests that take into account different terrains and scenarios in which the SGTV will be subjected to in actual operations. Maneuverability testing included demonstrating the SGTV's turning radius (true and swing area), and ability to closely approach a 90-degree wall and make a precision docking maneuver. Measurements were taken using a tape measure, feedback from the OAS sensors, and from GPS system. 


\subsubsection{Deviations from the Test Plan}

The testing was originally scheduled to be performed primarily at RAHCO International's facility in Spokane, Washington. Due to schedule problems and programmatic reasons, the testing was moved to a facility at the INEEL. This change of venue forced a significant number of changes in the test plan and procedure execution.

3.2.2.1 Operational and Transuranic Environment Evaluation. Due to the change of venue, the qualitative dust generation testing was not performed.

3.2.2.2 Throughput Testing. The REMEX and CTR gantry crane were not available to support actual waste loading and unloading of the SGTV. Throughput data was extrapolated from SGTV performance only.

3.2.2.3 Miscellaneous HMI, Safety, Vehicle and Control Performance Parameters. No attempt was made to specifically gather data on human interface issues of the SGTV and SGTV control systems. There are two reasons for this. First, severe time and budget constraints and lack of personnel support forced a decision on priorities that unfortunately meant having to eliminate this step. Second, new regulations were enacted that prohibit collection of human subject data (including human factors data) without submitting the process to an Institutional Review Board on human subject testing.

3.2.2.4 System Integration. System integration testing was to involve the SGTV and the REMEX with innovative end effector and the CTR gantry crane. The REMEX and CTR were not available or non-functional during the testing, therefore no formal system integration testing could be performed.

3.2.2.5 Precision and Accuracy of Navigation. Precision and accuracy testing was performed according to the test plan with modifications made only to accommodate the change of test venue.

3.2.2.6 SGTV UItrasonic Obstacle Avoidance. Ultrasonic obstacle avoidance testing was performed according to the test plan with some procedural changes to accommodate unanticipated test conditions.

3.2.2.7 Maneuverability. Due to the change in test venue, maneuverability testing was performed only on asphalt. 


\section{TEST RESULTS}

\subsection{Operational and Transuranic Environment Evaluation}

Operational and transuranic environment evaluations consist mainly of qualitative data. This data involves recording observations on visual dust generation, anomalous conditions, maintenance requirements, reparability, operational costs, and the overall reliability of the SGTV. Maintenance, repairability, and reliability observations were recorded through monitoring the frequency, type, and the approximate length of time for any required maintenance.

\subsubsection{Analysis}

Operation and transuranic environment evaluations use qualitative data. Qualitative data taken throughout testing will not be subject to analysis, but has been recorded and documented through log book entries, photographic evidence, and videotapes.

\subsubsection{Results and Evaluation}

4.1.2.1 Dust Generation. Due to the change of venue for the testing, formal dust generation evaluations were not made. The test area at the INEEL only offered operations on asphalt. Other soil and surface conditions were not available. However, before the official testing, development testing at RAHCO's facility in Spokane did offer a range of soil and surface conditions that were conducive to this evaluation.

The flat track grousers on the SGTV are designed to minimize dust generation during tracking, and soil plowing while turning. Dust generation is not a problem on asphalt or on dirt roads where either water or dust suppressants have been used $t$ prepare the surface. However, on soft soils where the edges of the grousers have an opportunity to dig in, they will carry some of the soil up onto the tracks. Under these conditions, significant amounts of dust will be generated, particularly at high speeds. Where the vehicle travels back and forth across the same path, and especially where it is making turns, even a prepared dirt road will soon be broken down by the tracks of such a large vehicle. Constant maintenance of the travel path will be required if the path is not asphalt or concrete.

Other than the flat grousers that were added during the FY-94 testing, the tracks are stock bulldozer tracks. They were selected and lengthened for use on this vehicle primarily for two reasons: First, the tracks provide the greatest load capacity and maneuverability, and second, they were available and inexpensive. All of the system requirements and operating scenarios for production equipment need to be better defined, but it is obvious that in almost any scenario, an improved track design could greatly reduce dust generation.

4.1.2.2 Anomalous Conditions. Only one anomalous mechanical event occurred. After approximately $100 \mathrm{hrs}$ of operation, the radiator on the SGTV developed a leak. This is discussed further under sections 4.1.2.3, "Reliability", and 4.1.2.4, "Maintenance." Several electronic system anomalies occurred during the testing. The most important (and annoying) was a frequent RS-485 network crash, which is discussed in further detail in section 4.1.2.3, 
"Reliability." Other anomalies that were noted in the electronics included occasional telemetry losses, on-board video dropout, erroneous OAS readings, and GPS/DR sensor errors.

Telemetry losses occurred randomly throughout the demonstration. These were not unexpected. A low-power spread-spectrum radio modem provided the telemetry communication link. Previous experience with these systems suggested that they were not completely reliable. However, for cost and licensing considerations, we elected to use this system. Interference from other electro-magnetic producing devices appeared to be the major cause of loss of telemetry. Multipath reflections also appeared to have a negative effect on telemetry especially when the vehicle was in the proximity of large steel structures. Time of day also had a large effect on quality of telemetry, with late afternoons and early evening often being very problematic. Careful placement of the telemetry receiver alleviated many of the problems. The communications were very reliable most of the time with only occasional short drop-outs.

Video signals suffered the same fate as the telemetry signals for some of the same reasons, although in general they were less of a problem. The video depended on a low-power line-ofsight FM transmitter/receiver. Reliable range of the SGTV (transmitter) to the receiver was fairly short (approximately $250 \mathrm{ft}$ ). The use of a high-gain unidirectional antenna in place of an omnidirectional antenna, and careful placement of the receiver, improved the video performance greatly. Some video flickering was experienced due to interference with another projects radio modem operating at the same frequency as the video signal.

On occasion, OAS sensors would report objects in the path that did not exist. This would result in stopping the vehicle for no apparent reason. In general, this was due to improperly set gains on the ultrasonic sensors. However on several occasions, for no apparent reason the OAS would allow the SGTV to follow a path with no difficulty only to command an emergency stop at approximately the same location every time, with no object in the path. The reason for this was not understood.

Errors from the GPS and DR sensors were expected, occurred with some regularity, and were even predictable, and in general should not be defined as anomalous. The control system was designed to handle such errors since this was the nature of these types of instruments. Once all the bugs were worked out of the system, and individual sensor performance was fully characterized, GPS/DR sensor errors were handled routinely. The one exception to this was an occasional GPS error that apparently caused the reported position of the SGTV to change by a large amount (so-called "Virtual Nebraska Bug" because the vehicle is briefly transported at the speed of light to an unknown position outside of the test area assumed to be somewhere in Nebraska). The cause of this problem was unknown, but appeared to be related to the manner in which the control software handled a bad GPS fix. Current navigation node code appears to correctly parse the raw data stream from the GPS (local zone data only composed of 4 , 80-character lines). Nevertheless, there continues to be an occasional bad fix sent to the VCCG computer. Software was written to capture this problem, but does not appear to be $100 \%$ effective. On rare occasions, it appears that this problem gets through, causing a very brief, large off-track error to be registered. This may have the effect of causing the SGTV to overcorrect for an error that doesn't exist thus actually creating an off-track error. This type of error was noted on a few occasions, but the control system quickly returned to normal and corrected for the error. The actual cause of this off-track error problem is not well understood and further investigation into this problem is warranted. 
4.1.2.3 Reliability. Mechanical reliability of the SGTV was excellent. The only serious mechanical problem involved a leaking radiator (see 4.1.2.4 "Maintenance"). This leak was located at the radiator's bottom mounting bracket and was caused by engine oscillation from the SGTV's movement over the terrain. The engine isolator mounts combined with the radiators isolators amplified this oscillation causing the bracket's welded joint to fatigue fail. The radiator was replaced and hard mounted to the frame.

Electronics reliability was also very good with one notable exception. At speeds exceeding approximately $3 \mathrm{ft} / \mathrm{sec}$ and angular rotation rates exceeding approximately $15 \mathrm{deg} / \mathrm{sec}$, frequent crashes of the RS-485 data network would occur. The network crashes could be duplicated at will by commanding the SGTV to exceed these thresholds. On the surface, the network crashes appear to be directly related to the effects of excessive vibration induced at the higher operating speeds. However, other network crashes occurred when the SGTV was operating in a relatively benign manner. Many of these other network crashes occurred at highly localized positions in the test area. Temporary fixes were implemented to automatically reset the network in the event of a crash, and operations were modified so as not to exceed the known threshold for network crashes. Network crashes that affected test results were noted in the log.

4.1.2.4 Maintenance. The SGTV mechanical systems require very little maintenance. The vehicle used standard industrial components. Some problems encountered reflect compromises in the design necessary for expediency and cost effectiveness of prototype hardware. The electronic systems required no maintenance once the system had been debugged and the final configuration was hard-programmed into the microcontroller.

A maintenance log was kept to record maintenance activities on the SGTV (Table 1). The items noted in this log are true maintenance tasks, and do not reflect structural or mechanical modifications made to the vehicle before or during testing to enhance performance or correct design deficiencies.

4.1.2.5 Ruggedness. With the exception of items previously noted, the structural mechanical systems on the SGTV were extremely rugged and should be able to withstand very harsh retrieval environments. The electronic components were individually designed to operate in a harsh environment, and, with the exception of the network problem, proved to be highly reliable under extremely harsh vibration conditions. Prototype equipment requires access for troubleshooting and debugging. A production system would use the same basic components, but packaging would be designed for the environment. In all probability, the electronic systems would be packed in a solid gel for protection and hardening.

\subsubsection{Discussion/Conclusion}

The testing demonstrated that the technology embodied in the SGTV is mature enough and reliable enough that it could be applied to a production design and deployed in a full-scale transuranic waste retrieval operation with only superficial modifications. 
Table 1. Mechanical maintenance log for the test.

\section{Type of}

Maintenance Frequency Scheduled Unscheduled Duration

\section{0-hr engine One time}

break-in oil

change

Lube track

bearings

Every 100
$\mathrm{hr}$

$\mathrm{X}$

$15 \mathrm{~min}$

Adjust track tension

As needed.

(Probably

every 100

hrs)
New radiator

Oil filter

Air filter
$\mathrm{X}$

$\mathrm{X}$
Comments

Changing the oil is a simple operation. Access is good. Breakin oil needs to be changed after 100 hrs. Otherwise, changing the oil depends on operating conditions and manufacturer's recommendation.

Zerks for track lubrication are easily accessible, and lubrication procedure is simple. Sealed bearings, or automatic lubrication system should be considered for production hardware.

Checking and adjusting the track tension should be a scheduled operation, but was not done due to the short duration of the testing and the limited operating time on the vehicle. Production hardware designs should require significantly less maintenance.

$\mathrm{X} \quad 1 \mathrm{hr}$

The radiator had to be replaced after approximately $100 \mathrm{hrs}$ of operation. Excessive vibration caused the radiator to develop a leak at an attach point. Improvements in the engine mounting scheme are required to alleviate this situation.

Changing the oil filter is a simple operation. Access is good. The oil filter should be changed with each oil change.

Changing the air filter is a simple operation. Access is good. 
Many improvements to the basic SGTV were realized when compared to the state of the vehicle in FY-94. A new and more powerful engine was installed. A completely new hydraulic system was designed. Hydraulic lines were hard-plumbed and routed through pipe supports. Hydraulic valves were centrally located and mounted on a platform for easy installation and maintenance. The SGTV was designed to be easier to build, maintain, and to provide higher reliability and increased performance. The SGTV is essentially designed and built to heavy equipment industrial standards.

The equipment was designed with operation in a TRU environment in mind, but the fact remains that this is prototype equipment with budget and schedule constraints. No attempt to design the equipment for high radiation environments or ease of decontamination was made. Additional design features would be required for production deployment in a TRU environment. Some suggestions for improvements and design requirements are provided in Section 5.

The effects of vibration were the single most important factor in the ruggedness of the SGTV mechanical and electrical systems. Incremental improvements are indicated for many of the systems on the SGTV for various reasons. Ruggedization of the electronic components is necessary, but reduction of the induced vibration load from the vehicle must be one of the highest priorities for a production design to ensure a more reliable system.

\subsection{Throughput}

The maximum throughput of the SGTV was determined by following the test procedures outlined in the Test Plan with the exceptions previously noted. Since there was no method to load waste or waste modules into the SGTV the time for the loading and unloading procedures could not be measured, nor could the procedures be documented. Track distances were measured along with the complexities involved with the vehicle's path. This measurement of time to distance allows for calculation of the possible throughput of the SGTV with some assumptions. Test data was gathered in both telerobotic and self-guided control modes for comparison.

\subsubsection{Analysis}

Analysis of the throughput of the system includes calculations of the mean and standard deviation of the times taken for transportation for each testing cycle. Qualitative data such as anomalous conditions were recorded and documented through log book entries. Calculations for determining the mean and standard deviation are as follows:

Mean: Average time for the SGTV to transit from the start to the end points of a path.

$$
\bar{X}=\frac{\sum_{i=1}^{n} X_{i}}{n}
$$

where

$$
X_{i} \quad=\quad \text { a time measurement }
$$



$\bar{X} \quad=\quad$ the mean of time measurements
$n \quad=\quad$ number of times the test was timed.

Standard Deviation: Average deviation of the observations from each other.

$$
S=\sqrt{\frac{\sum_{i=1}^{n}\left(X_{i}-\bar{X}\right)^{2}}{n-1}}
$$

where

$$
\begin{array}{lll}
X_{i} & = & \text { a time measurement } \\
\bar{X} & = & \text { the mean of time measurements } \\
S & = & \text { the standard deviation } \\
n & = & \text { number of times the test was timed. }
\end{array}
$$

\subsubsection{Results and Evaluation}

The data for the throughput calculations was gathered in conjunction with the precision and accuracy testing and are presented below for the paths that were designed for those tests. Three paths were defined for the testing. Each path includes a start (S) and end (E) point with flyby (F) and pause (P) points in-between (see Figures 17, 18, and 19). Some of the flyby points (e.g., point $2 \mathrm{~F}$ in Figure 17) are what are known as smooth flybys where the SGTV is commanded to make a smooth transition turn between the navigation path segments. At the other flyby points, the vehicle stops and makes a pure rotation turn, then proceeds to the next waypoint. At pause points, the vehicle will pause until the SGTV operator commands it to proceed. In all cases, the maximum commanded velocity $\left(V_{\max }\right)$ for the SGTV was $2.5 \mathrm{ft} / \mathrm{s}$ and the maximum turning rate $\left(\omega_{\max }\right)$ was $10 \mathrm{deg} / \mathrm{s}$. The size of each path and the area for maneuvering the vehicle were highly constrained by the limited space available for the demonstration. For this reason and others (which will be detailed later), the speed of the SGTV was limited to half its rated capacity.

Tables 2,3 , and 4 provide the raw data and the mean and standard deviation calculations for each of the three self-guided test paths. Time measurements were performed manually with a stop watch as a comparison for the data logged by the VCCG. Both manual and VCCG time measurements are reported. Notes regarding anomalies that may have affected the SGTV transit time are provided in the tables. Network and telemetry errors can cause the vehicle to pause until the error is corrected. Most of the time the error is self-correcting within less than one second, but on occasion a network error requires that the onboard computer be rebooted. Depending on the severity of the error, the vehicle may pause until the error is corrected, at which time the operator would command the vehicle to resume tracking. Telemetry errors appear to be mostly related to radio interference. Network errors are much more complex and are discussed elsewhere in this document. Time measurements reflect actual transit time and do not include downtime due to errors. 


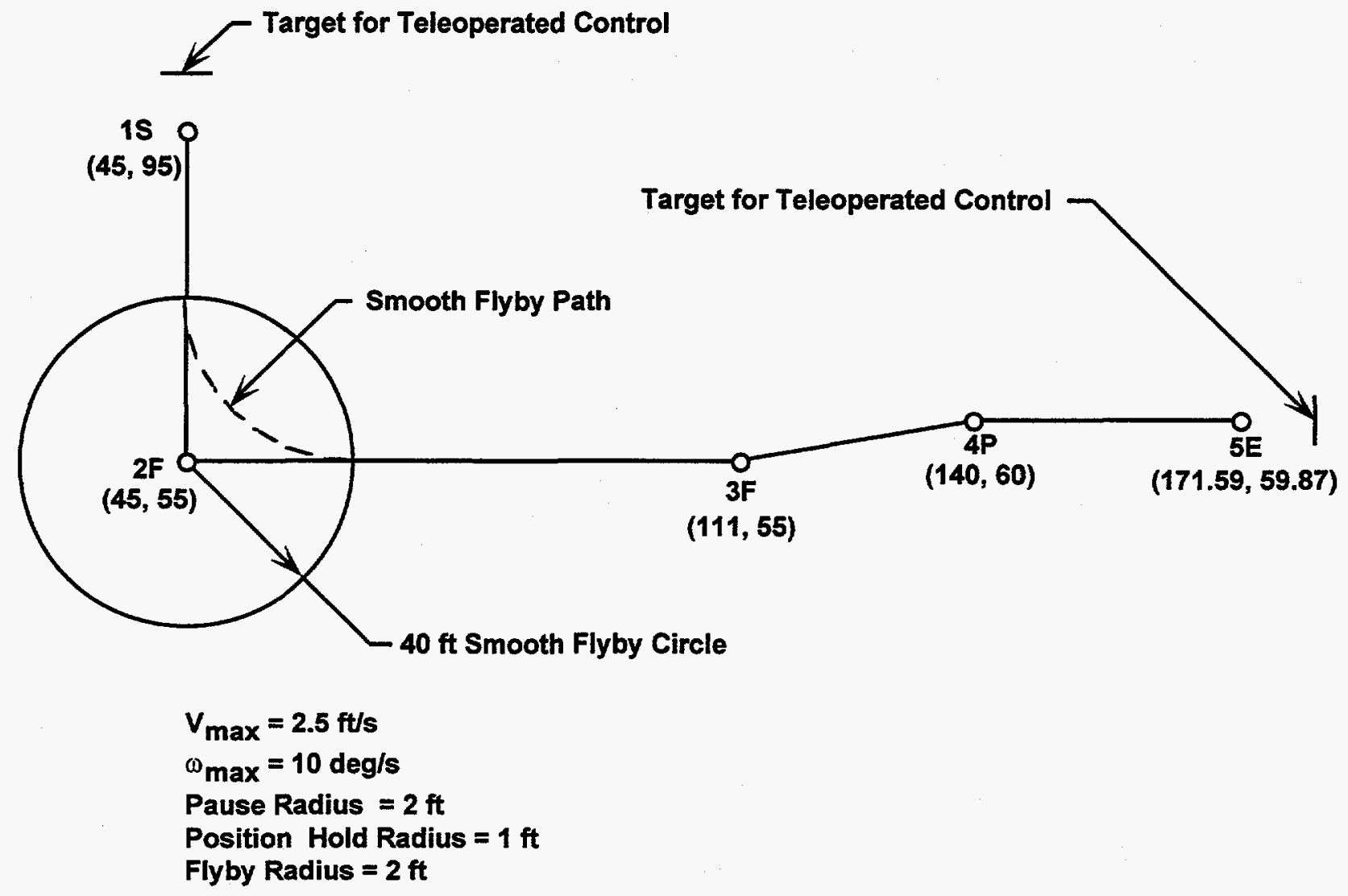

Figure 17. Path 1 is a simple path starting at point $1 \mathrm{~S}$ and ending at point $5 \mathrm{E}$. The SGTV proceeds along the path from $1 \mathrm{~S}$ to $5 \mathrm{E}$ and then backtracks along the same path from $5 \mathrm{E}$ to $1 \mathrm{~S}$. This type of path could be used where a single SGTV is transferring waste between the digface and a transfer station, or where limited space does not allow for maneuvering in a race track pattern. The total length of travel for path 1 is $316 \mathrm{ft}$ (158 ft each direction). 


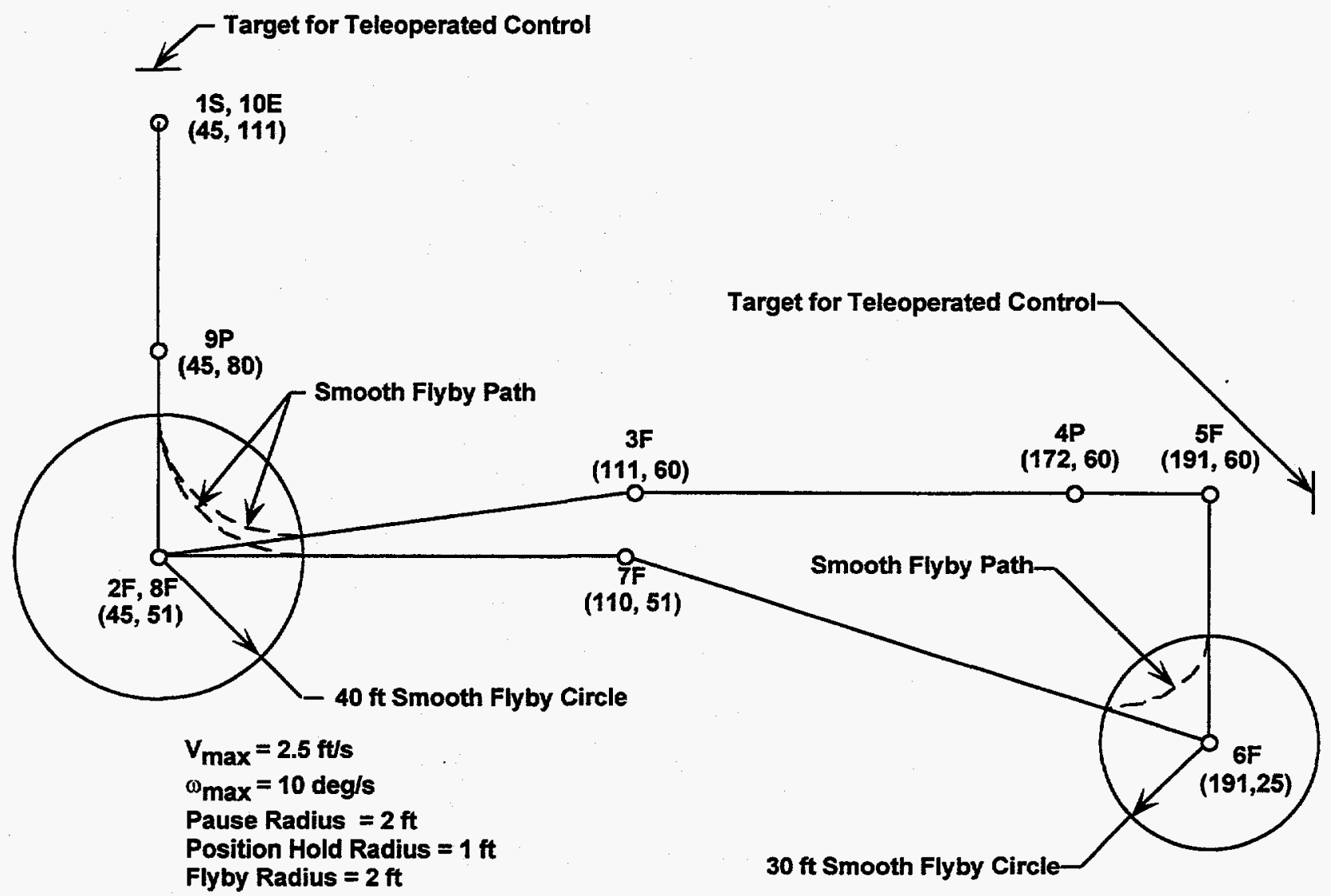

Figure 18. Path 2 is a more complicated path, starting at point $1 \mathrm{~S}$ and ending at the same location point $10 \mathrm{E}$. This path includes three smooth flybys and one 90 -degree pure rotation turn. The SGTV proceeds from point $1 \mathrm{~S}$ to point $10 \mathrm{E}$ in a racetrack pattern, tracing its own path into and out of the start/end point. This racetrack pattern, could be used where multiple SGTVs are in continuous operation. Waste would be transferred from the digface to the SGTV at a pause point, such as point $4 \mathrm{P}$, somewhere along the path of the vehicle. Timing would be important to avoid conflicts between multiple vehicles using this path, however a slight modification placing the start and end points within the loop of the racetrack would reduce this problem. The total length of travel for path 2 is $408 \mathrm{ft}$. 


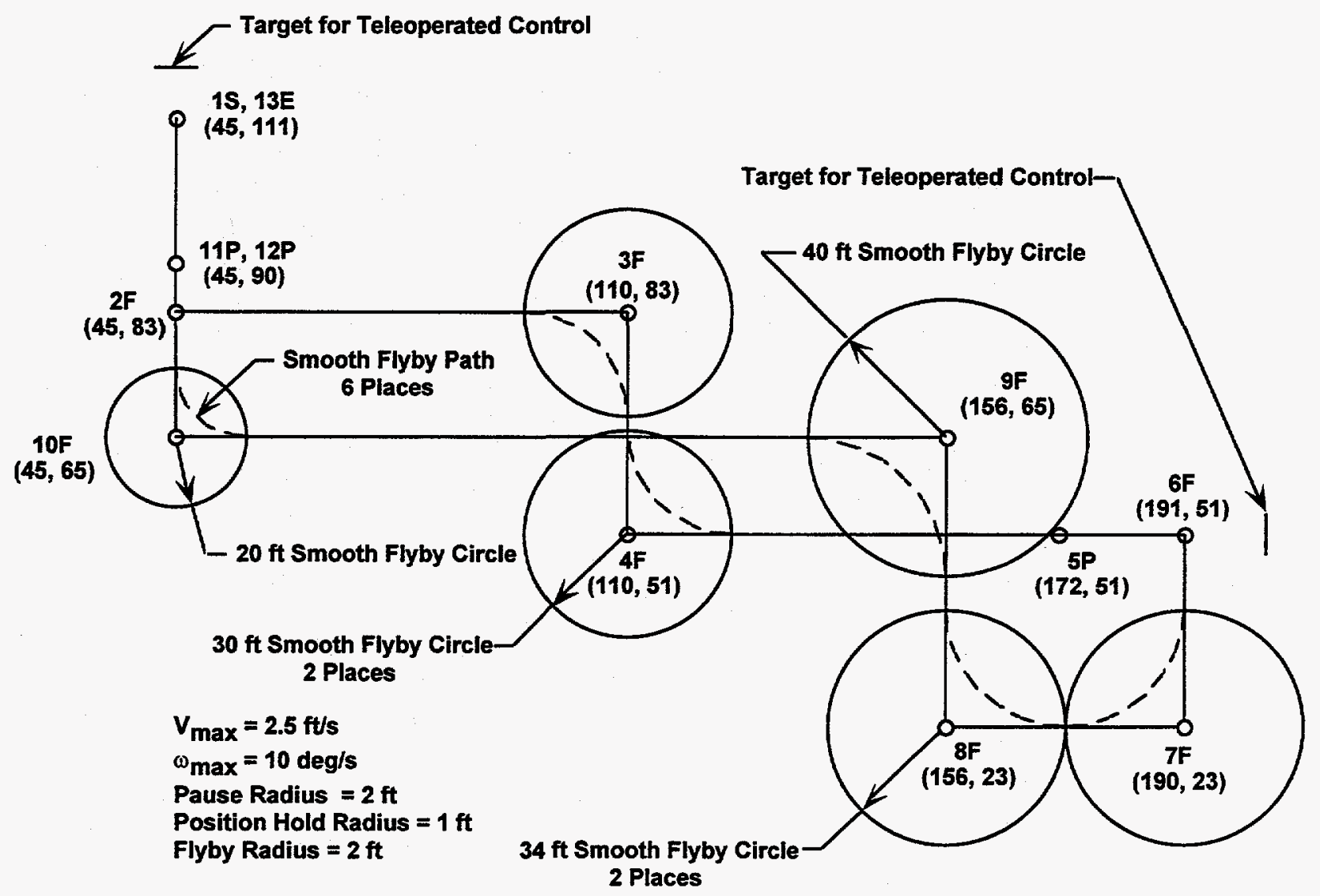

Figure 19. Path 3 is a very complicated path starting at point $1 \mathrm{~S}$ and ending at the same location point $13 \mathrm{E}$. This path includes six smooth flybys and two 90 -degree pure rotation turns. The SGTV proceeds from point $1 \mathrm{~S}$ to point $13 \mathrm{E}$ in a racetrack pattern tracing its own path into and out of the start/end point. This path was designed solely to challenge the navigation of the vehicle and increase the time required for transit within the limited space of the test site. It does not necessarily reflect a typical path that would be used in a retrieval. The total length of travel for path 3 is $428 \mathrm{ft}$. 
Table 2. Times to navigate test path number 1.

\begin{tabular}{|c|c|c|c|c|}
\hline $\begin{array}{l}\text { Run } \\
\text { Number }\end{array}$ & $\begin{array}{c}\text { Path/Heading } \\
\text { ( } \mathrm{F}=\text { Forward } \\
\mathrm{R}=\text { Reverse) }\end{array}$ & $\begin{array}{c}\text { Time (s) } \\
\text { Manual } \\
\text { Measurement }\end{array}$ & $\begin{array}{c}\text { Time (s) } \\
\text { Computer } \\
\text { Measurement }\end{array}$ & Notes \\
\hline $\mathrm{KS}$ & $1 \mathrm{~S}-5 \mathrm{E} / \mathrm{F}$ & na & 176 & Kinematic Simulation \\
\hline KS & 5E-1S/R & na & 177 & Kinematic Simulation \\
\hline 1 & SE-1S/R & * & 130 & \\
\hline 2 & 1S-5E/F & * & 133 & \\
\hline 3 & $5 \mathrm{E}-1 \mathrm{~S} / \mathrm{R}$ & * & 142 & 1 Telemetry Error \\
\hline 4 & $1 \mathrm{~S}-5 \mathrm{E} / \mathrm{F}$ & * & 135 & 1 Telemetry Error, 1 Network Error \\
\hline 5 & 5E-1S/R & * & 135 & \\
\hline 6 & $1 \mathrm{~S}-5 \mathrm{E} / \mathrm{F}$ & * & 146 & 5 Network Errors (all reset) account for $\sim 10 \mathrm{~s}$ \\
\hline 7 & 5E-1S/R & * & 132 & 1 Network Error \\
\hline 8 & $1 \mathrm{~S}-5 \mathrm{E} / \mathrm{F}$ & $*$ & 134 & \\
\hline 9 & SE-1S/R & 128 & 132 & \\
\hline \multirow[t]{3}{*}{10} & $1 \mathrm{~S}-5 \mathrm{E} / \mathrm{F}$ & 130 & 127 & \\
\hline & Mean & na & 134.6 & $\begin{array}{l}\text { Mean time to complete path from start to finish } \\
\text { (either direction) for the } 10 \text { trials. }\end{array}$ \\
\hline & Std Deviation & na & 5.6 & \\
\hline
\end{tabular}

Table 3. Times to navigate test path number 2.

\begin{tabular}{|c|c|c|c|c|}
\hline $\begin{array}{l}\text { Run } \\
\text { Number }\end{array}$ & $\begin{array}{c}\text { Path/Heading } \\
\text { (F=Forward } \\
\mathrm{R}=\text { Reverse) }\end{array}$ & $\begin{array}{c}\text { Time (s) } \\
\text { Manual } \\
\text { Measurement }\end{array}$ & $\begin{array}{c}\text { Time (s) } \\
\text { Computer } \\
\text { Measurement }\end{array}$ & Notes \\
\hline KS & $1 \mathrm{~S}-10 \mathrm{E} / \mathrm{F}$ & na & 608 & Kinematic Simulation \\
\hline 1 & $1 \mathrm{~S}-10 \mathrm{E} / \mathrm{R}$ & 349 & 345 & 1 Network Error \\
\hline 2 & $1 \mathrm{~S}-10 \mathrm{E} / \mathrm{F}$ & 354 & 349 & \\
\hline 3 & $1 S-10 E / R$ & 356 & 347 & 1 Network Error \\
\hline 4 & $1 \mathrm{~S}-10 \mathrm{E} / \mathrm{F}$ & 359 & 360 & 1 Telemetry Error \\
\hline 5 & $1 \mathrm{~S}-10 \mathrm{E} / \mathrm{R}$ & 359 & 355 & \\
\hline 6 & $1 \mathrm{~S}-10 \mathrm{E} / \mathrm{F}$ & 364 & 349 & 1 Telemetry Error \\
\hline 7 & $1 \mathrm{~S}-10 \mathrm{E} / \mathrm{R}$ & 354 & 352 & 1 Network Error \\
\hline 8 & $1 \mathrm{~S}-10 \mathrm{E} / \mathrm{F}$ & 350 & 348 & 3 Telemetry Errors \\
\hline 9 & $1 S-10 E / R$ & 373 & 370 & 1 Network Error \\
\hline \multirow[t]{3}{*}{10} & $1 \mathrm{~S}-10 \mathrm{E} / \mathrm{F}$ & 363 & 362 & 2 Telemetry Errors \\
\hline & Mean & 358 & 354 & $\begin{array}{l}\text { Mean time to complete path from start to finish for } \\
\text { the } 10 \text { trials. }\end{array}$ \\
\hline & Std Deviation & 7.2 & 8.0 & \\
\hline
\end{tabular}


Table 4. Times to navigate test path number 3.

\begin{tabular}{|c|c|c|c|c|}
\hline $\begin{array}{c}\text { Run } \\
\text { Number }\end{array}$ & $\begin{array}{l}\text { Path/Heading } \\
\text { (F=Forward } \\
\mathrm{R}=\text { Reverse) }\end{array}$ & $\begin{array}{c}\text { Time (s) } \\
\text { Manual } \\
\text { Measurement }\end{array}$ & $\begin{array}{c}\text { Time (s) } \\
\text { Computer } \\
\text { Measurement }\end{array}$ & Notes \\
\hline $\mathrm{KS}$ & $1 \mathrm{~S}-13 \mathrm{E} / \mathrm{F}$ & na & 560 & Kinematic Simulation \\
\hline 1 & 1S-13E/F & 425 & 457 & Unknown E-Stop after pt 2 (probably OAS) \\
\hline 2 & 1S-13E/R & 414 & 414 & 1 Telemetry Error \\
\hline 3 & $1 \mathrm{~S}-13 \mathrm{E} / \mathrm{F}$ & 413 & 415 & 1 Telemetry Error \\
\hline 4 & $1 \mathrm{~S}-13 \mathrm{E} / \mathrm{R}$ & 420 & 421 & 2 Telemetry Errors \\
\hline 5 & $1 \mathrm{~S}-13 \mathrm{E} / \mathrm{F}$ & 423 & 420 & 1 Telemetry 1 OAS Error \\
\hline 6 & $1 S-13 E / R$ & 409 & 407 & 2 Network and 7 GPS Errors \\
\hline 7 & $1 \mathrm{~S}-13 \mathrm{E} / \mathrm{F}$ & 415 & 418 & 1 Network Error (Required Reboot) \\
\hline 8 & IS-13E/R & 414 & 410 & 1 Network Error \\
\hline 9 & 1S-13E/R & 415 & 425 & 1 Network 1 Telemetry Error \\
\hline \multirow[t]{3}{*}{10} & $1 \mathrm{~S}-13 \mathrm{E} / \mathrm{F}$ & 431 & 424 & 2 Network Errors (1 Required reboot) \\
\hline & Mean & 418 & 421 & $\begin{array}{l}\text { Mean time to complete path from start to } \\
\text { finish for the } 10 \text { trials. }\end{array}$ \\
\hline & Std Deviation & 6.7 & 13.9 & \\
\hline
\end{tabular}

To calculate a throughput rate for the vehicle, it is necessary to make some assumptions concerning loading and unloading times and capacity of the SGTV. Those assumptions are as follows:

Load capacity - While the SGTV is capable of carrying objects up to $4 \times 4 \times 8 \mathrm{ft}$ and weighing well in excess of $9000 \mathrm{lb}$, its primary cargo is designed to be ITMs full of soil and waste. The SGTV can carry two ITMs in the waste transport container with $.75 \mathrm{yd}^{3}$ in each container. A conservative approach would be to estimate that each ITM is filled to $75 \%$ of capacity giving a total load of $1.125 \mathrm{yd}^{3}$ per load.

Loading time - Based on previous demonstrations, an experienced operator with a remote-operated excavator can dig and load an ITM in about 2 minutes. Since digging operations would be continuous during transportation, it can be assumed that at least one of the two ITMs is ready to be loaded upon arrival of the SGTV. To load two ITMs into the vehicle, estimate 3 minutes.

Unloading time - The vehicle is designed for roll-on roll-off of WTCs and ITMs. The back-end equipment to perform this procedure does not exist, and therefore could not be tested. The operation is simple and should be able to be accomplished in a fully automated manner in about 2 minutes. 
Time to open and close the WTC lid - This time was measured during the demonstration. The average time to open the lid was 10.75 seconds. The average time to close the lid was 12.2 seconds.

To calculate a throughput rate for the SGTV based upon the data in tables 2, 3, and 4, along with the assumptions stated above, the following formula was used:

$M=\left\{V_{T} /\left(T_{1}+T_{2}+T_{3}+T_{4}+T_{5}\right)\right\} \times(28,800 s /$ day $)$

where

\begin{tabular}{|c|c|c|}
\hline M & $=$ & throughput rate in $\mathrm{yd}^{3}$ per day \\
\hline $\mathrm{V}_{\mathrm{T}}$ & $=$ & volume of waste transported in a cycle \\
\hline $\mathrm{T}_{1}$ & $=$ & time to transit full cycle (start to end and return as necessary) \\
\hline $\mathrm{T}_{2}$ & $=$ & time to open WTC lid \\
\hline $\mathrm{T}_{3}$ & $=$ & time to load the SGTV with two ITMs \\
\hline $\mathrm{T}_{4}$ & $=$ & time to close WTC lid \\
\hline$T_{5}$ & $=$ & time to unload the WTC and put new WTC and ITMs on SGTV \\
\hline
\end{tabular}

\subsubsection{Discussion/Conclusion}

The throughput rates for the SGTV shown in Table 5 depend most on the length of travel required to transport the waste and the complexity of the path. The load capacity and the other variables previously discussed should be mostly constant. The three paths used in the test were designed to exercise these variables in a manner believed to be representative of a realistic scenario for a hot spot retrieval in a TRU waste pit. Restrictions placed on operations due to the limited availability of space for safe maneuvering, and the vibration induced network crashes caused throughput rates to drop below the goal of $80 \mathrm{yd}^{3} /$ day for typical operating scenarios. Increasing the vehicle's speed to its maximum design of $5 \mathrm{ft} / \mathrm{sec}$ (linear) would provide greatly improved throughput rates. Of course, one of the most important aspects of the design of the SGTV is that since it is self-guided, multiple vehicles can be easily deployed to increase the production rate to any desired level with minimal impact to production costs.

Table 5. The calculated throughput rates for the SGTV for each path in Figures 17, 18, and 19. Path 1 is a single path on which the SGTV backtracks to deliver waste, therefore the time for this path is the sum of the forward and reverse tracks. Paths 2 and 3 are closed loops.

\begin{tabular}{cccc}
\hline Path & $\begin{array}{c}\text { Mean Time for the SGTV } \\
\text { to Complete Path }(\mathrm{sec})\end{array}$ & $\begin{array}{c}\text { Volume of Waste Transported per } \\
\text { Load in the SGTV (yd3) }\end{array}$ & $\begin{array}{c}\text { Throughput } \\
\text { (yd3/day) }\end{array}$ \\
\hline 1 & 592 & 1.125 & 51.7 \\
2 & 677 & 1.125 & 47.8 \\
3 & 743 & 1.125 & 43.6 \\
\hline
\end{tabular}




\subsection{Miscellaneous HMI, Safety, and Vehicle and Control System Performance}

The data from a number of tests involving safety, human interface, vehicle performance, mission planning and VCCG graphical user interface are presented here.

\subsubsection{Analysis}

Most of the data taken with respect to HMI, safety, and vehicle and control performance is qualitative and not subject to analytical inspection. Quantitative analysis, when necessary, will involve simply reporting the data or minimal statistical analysis (such as calculating the mean and standard deviation).

\subsubsection{Results and Evaluation}

4.3.1.1 Human Factors. No specific data were gathered on human factors issues for the reasons previously stated. Comments on human factors as relates to the design and use of the system can be found in Section 5, "Discussion, Conclusions, and Recommendations."

\subsubsection{Miscellaneous Vehicle/Control System Performance}

Speed and acceleration

A series of six speed and acceleration tests were performed to examine the maximum performance of the vehicle using the control gains designed into the system. The absolute maximum performance parameters of the SGTV were not established. The test was performed in teleoperated direct track mode. Table 6 contains the data taken during the test. The total time was obtained with a stopwatch, and the start and end location were obtained from the on-board GPS. The top speed of the SGTV with the current control gains is $6 \mathrm{ft} / \mathrm{s}(360 \mathrm{ft} / \mathrm{min}$ or approximately 4 miles/hr). The SGTV accelerates and decelerates rapidly reaching maximum speed and stopping from maximum speed in approximately 1.5 seconds (see Figure 20).

Table 6. Results of speed and acceleration testing.

\begin{tabular}{lcccc}
\hline $\begin{array}{c}\text { Trial Number } \\
\text { and Direction } \\
\text { of Travel }\end{array}$ & $\begin{array}{c}\text { Starting Position } \\
\text { (GPS x,y location) }\end{array}$ & $\begin{array}{c}\text { Final Position } \\
\text { (GPS x,y location) }\end{array}$ & $\begin{array}{c}\text { Total Time } \\
\text { (s) }\end{array}$ & $\begin{array}{c}\text { Top Speed } \\
\text { (ft/s) }\end{array}$ \\
\hline 1-Reverse & $178.33,45.89$ & $135.18,52.75$ & 8.90 & 6 \\
2-Forward & $135.45,52.52$ & $179.77,56.32$ & 9.14 & 6 \\
3-Reverse & $179.54,56.32$ & $137.64,55.31$ & 8.64 & 6 \\
4-Forward & $137.77,55.37$ & $181.31,54.12$ & 8.66 & 6 \\
5-Reverse & $181.31,54.12$ & $136.56,53.8$ & 9.06 & 6 \\
6-Forward & $136.56,53.8$ & $184.92,54.16$ & 9.71 & 6 \\
\hline
\end{tabular}




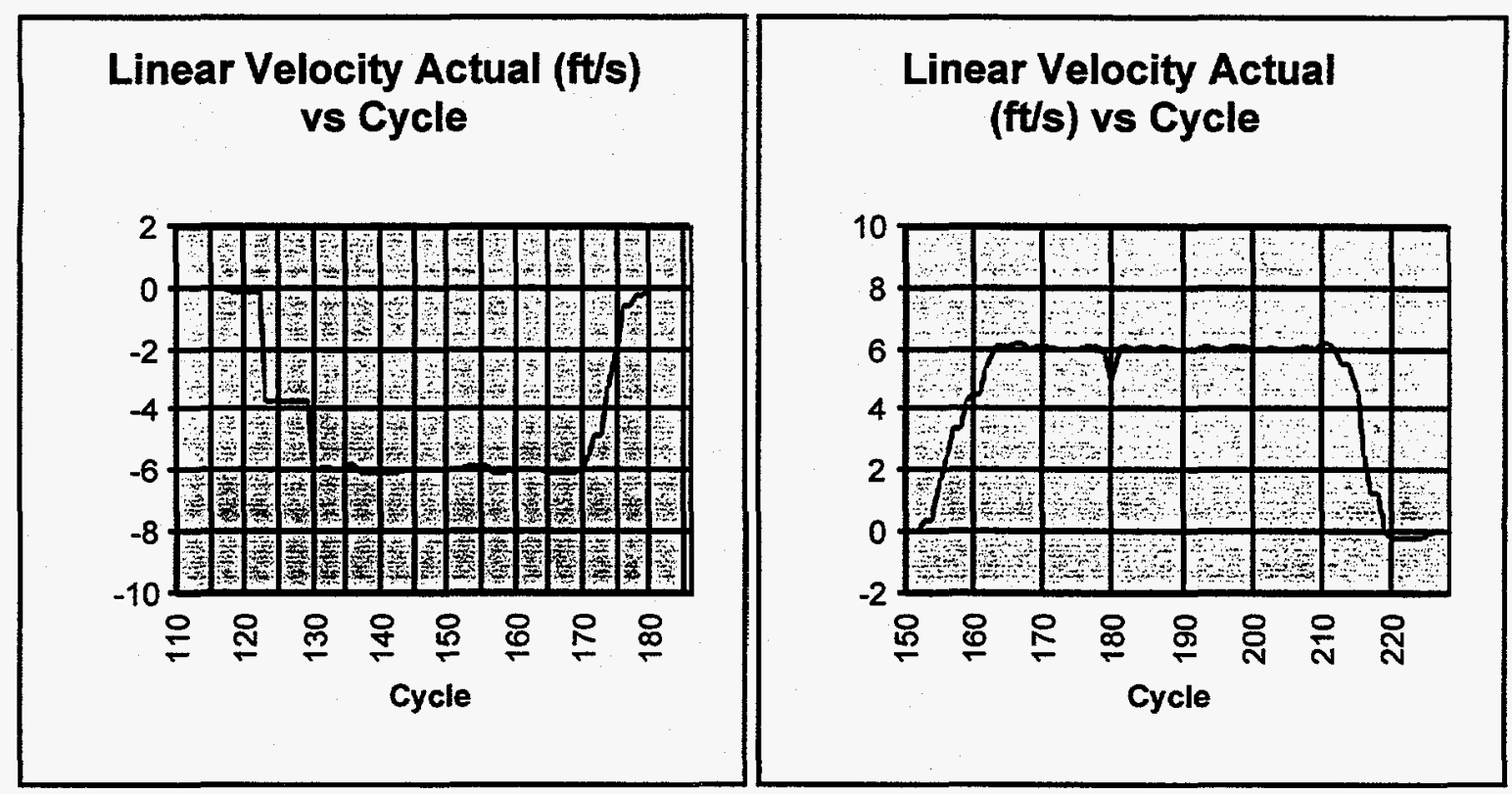

Figure 20. The SGTV rapidly accelerates to and decelerates from its maximum speed. The first curve is from trial number 1 . The reverse sign on the linear velocity is due to the direction of travel (reverse) of the SGTV. The second curve is from trial number 6. Note that each cycle is approximately $155 \mathrm{~ms}$.

\section{System health monitoring}

There are 12 system health monitoring/status signals the SGTV sends to the VCCG for advising the operator on vehicle status. Some of these signals (GPS error detection, network error, etc.) interface with the control system to stop the vehicle in the event of a non-acceptable condition. Table 7 provides the results of the functional test of the system health monitoring signals.

Table 7. The results of the testing of the system health monitoring features.

\begin{tabular}{lll}
\hline \multicolumn{1}{c}{ Indicator } & \multicolumn{1}{c}{ Function } & \multicolumn{1}{c}{ Notes } \\
\hline Communications Link & $\begin{array}{l}\text { Indicates status of RS-232 link to } \\
\text { the network router. }\end{array}$ & $\begin{array}{l}\text { Not tested. This is item was not part } \\
\text { of the specification. It was added by } \\
\text { Spar for debugging purposes. }\end{array}$ \\
Telemetry & $\begin{array}{l}\text { Indicates status of telemetry } \\
\text { connection between SGTV and } \\
\text { VCCG }\end{array}$ & $\begin{array}{l}\text { Functional. Will command vehicle to } \\
\text { stop on extended loss of telemetry. }\end{array}$ \\
Network & $\begin{array}{l}\text { Indicates status of the RS-485 } \\
\text { network on-board the SGTV }\end{array}$ & $\begin{array}{l}\text { Functional. Alerts operator and } \\
\text { commands vehicle to stop on extended } \\
\text { loss of network. }\end{array}$ \\
\hline
\end{tabular}


Table 7. (continued).

\begin{tabular}{|c|c|c|}
\hline Indicator & Function & Notes \\
\hline Engine oil pressure & Low-pressure warning light & Functional. Alerts operator. \\
\hline Engine temperature & $\begin{array}{l}\text { High engine oil temperature } \\
\text { warning light }\end{array}$ & Functional. Alerts operator. \\
\hline Battery voltage & Low battery-voltage indicator & Not tested. Alerts operator. \\
\hline $\begin{array}{l}\text { Hydraulic oil } \\
\text { temperature }\end{array}$ & High-temperature warning & Not tested. Alerts operator. \\
\hline $\begin{array}{l}\text { Electronics box } \\
\text { temperature }\end{array}$ & High-temperature warning & Functional. Alerts operator. \\
\hline Container latch status & Container latch status indicator & $\begin{array}{l}\text { Functional. Alerts operator that } \\
\text { container latch is not engaged. }\end{array}$ \\
\hline Container lid status & Container lid status indicator & $\begin{array}{l}\text { Functional. Alerts operator that } \\
\text { container lid is may not be closed. }\end{array}$ \\
\hline Compass status & $\begin{array}{l}\text { Indicates if compass is sending } \\
\text { error message }\end{array}$ & $\begin{array}{l}\text { Functional. Alerts operator and } \\
\text { commands vehicle to stop on extended } \\
\text { error. (Compass is too sensitive to } \\
\text { inclinometer errors and reports many } \\
\text { short duration errors). }\end{array}$ \\
\hline GPS status & $\begin{array}{l}\text { Indicates if GPS system is not } \\
\text { updating its position }\end{array}$ & $\begin{array}{l}\text { Functional. Alerts operator and } \\
\text { commands vehicle to stop on extended } \\
\text { error. }\end{array}$ \\
\hline
\end{tabular}

\subsubsection{Safety}

\section{Hydraulics enable/emergency stop system}

A functional test was performed to checkout all of the hydraulics enable and emergency stop functions of the SGTV system design. Table 8 provides the data from this test.

\section{System response anomalies}

Two true "anomalies" occurred during the testing and development. An anomaly as described here is response from the SGTV that was significantly different than the commanded or desired response that cannot be easily explained. 
Table 8. All available emergency stop functions were tested to ensure they performed as designed. In addition to the formal test, many of the emergency stop functions (especially the hand-held unit and the teleoperator's control station hydraulics cut-off switch) were tested at least daily and most of the time several times a day to ensure that they were always functional. This was especially important during the development phase of the project to ensure that hardware and software changes had no effect on critical safety systems.

\begin{tabular}{|c|c|c|c|}
\hline Function & Pass & Fail & Notes \\
\hline $\begin{array}{l}\text { On-board emergency } \\
\text { stop switch }\end{array}$ & $X$ & & $\begin{array}{l}\text { Cut hydraulics and fuel enable. Engine died } \\
\text { immediately. }\end{array}$ \\
\hline $\begin{array}{l}\text { Pendant-mounted } \\
\text { emergency stop switch }\end{array}$ & $\mathrm{X}$ & & $\begin{array}{l}\text { Cut hydraulics and fuel enable. Engine died } \\
\text { immediately. }\end{array}$ \\
\hline \multirow{5}{*}{$\begin{array}{l}\text { Hand-held radio } \\
\text { emergency stop switch } \\
\text { (Remtron) }\end{array}$} & $\mathrm{X}$ & & $\begin{array}{l}\text { Checked four functional parameters of the Remtron } \\
\text { device: }\end{array}$ \\
\hline & & & 1)"Emergency Stop" button depressed \\
\hline & & & 2) Power turned off for more than $10 \mathrm{sec}$ \\
\hline & & & 3) Lost telemetry for more than $10 \mathrm{sec}$ \\
\hline & & & $\begin{array}{l}\text { 4) Remtron inclined more than } 30^{\circ} \text { for } \\
10 \mathrm{sec}\end{array}$ \\
\hline $\begin{array}{l}\text { Control station } \\
\text { hydraulics off switch }\end{array}$ & $\mathrm{X}$ & & $\begin{array}{l}\text { Disabled power to the hydraulic valves stopping the } \\
\text { vehicle. Engine continued to run as expected. }\end{array}$ \\
\hline \multirow{7}{*}{$\begin{array}{l}\text { VCCG issues zero rate } \\
\text { command and/or on- } \\
\text { board computer issues } \\
\text { E-stop }\end{array}$} & $\mathrm{X}$ & & $\begin{array}{l}\text { Disabled power to the hydraulic valves stopping the } \\
\text { vehicle. Engine continued to run as expected. Checked } \\
\text { several methods for doing this, including: }\end{array}$ \\
\hline & & & 1) OAS commanded emergency stop \\
\hline & & & 2) Loss of data network \\
\hline & & & 3) Loss of telemetry \\
\hline & & & $\begin{array}{l}\text { 4) Sustained (more than } 3 \text { cycles) GPS or } \\
\text { Compass error }\end{array}$ \\
\hline & & & 5) Manual command to stop from VCCG \\
\hline & & & 6) Off-track error exceeds tolerance limit \\
\hline
\end{tabular}

Early in the development, just after the control system electronics were installed on the SGTV, the first system response anomaly occurred. During routine checkout, a full-power, high-speed pure rotation turn (SGTV turning about its own axis) was being executed in telerobotic mode from the remote station. When the operator completed the turn and released the joystick, the SGTV continued to make the turn, effectively sitting and spinning in place. The operator hit the hydraulics off button on the control panel immediately stopping the vehicle. Operations were continued after a brief investigation with no anomalies of this nature occurring during the development and testing (approximately 3 months) of the SGTV. 
The other anomaly was alluded to in section 4.1.2.2, "Anomalous Conditions." On occasion, in both self-guided and teleoperated mode, the SGTV will make a sudden uncommanded turn for a split second, and then return to normal. In self-guided mode, this has the effect of introducing a slight off-track error that is quickly corrected by the VCCG. In teleoperated mode, the operator will notice a slight change of direction of the SGTV that requires a minor course correction. The error only lasts for a fraction of a second and occurs very rarely. The error does not pose a safety threat to the operations of the SGTV, but is a concern when trying to maintain highly precise navigation. Because of the highly intermittent nature of the problem and a lack of time to properly investigate it, no cause for this anomaly has been determined.

\section{Safety system malfunctions}

There were no safety system malfunctions observed during the development or testing of the SGTV.

\section{System response to operator errors}

In self-guided or teleoperated mode, the OAS system is looking in the direction of travel. On one occasion during the testing, an operator driving the vehicle in teleoperated mode attempted to turn the vehicle too close to an object (wooden stanchion). The rear of the vehicle swung about and contacted the object. The rear OAS sensors were not operating, and because of the swing angle would probably not have detected the object in time to prevent the collision. The safety observer depressed the emergency stop button on the hand-held emergency stop radio and stopped the vehicle.

This type of incident is much less likely to occur in self-guided mode. Assuming the mission planning database is kept up-to-date, potential collisions of this kind can be predicted and planned out of the path via the use of the mission planning software's collision detection routines and by performing a kinematic simulation before moving the vehicle. The OAS system should handle most all of the potential collisions due to unanticipated objects entering the path of the vehicle.

\subsubsection{Mission Path Planning/Vehicle Command Control Generator Performance}

\section{No-go zone creation}

A no-go zone map was created using the mission planning software per the procedure provided by Spar. The basic map for the test site had been previously loaded into the computer. The mission planning software has a user definable coordinate system. For this test, a local coordinate system was established and used to define the no-go zones and the SGTV navigation paths. Some of the other features of the mission planning software for no-go zone creation include capability to establish a buffer zone around obstacles as an extra measure of protection, capability to save multiple no-go zone data files, and the capability to load previously stored nogo zones from disk and modify them. 


\section{Path creation}

Three paths were created that were designed to be representative of paths that could be used in an actual retrieval. The three paths were designated TP1, TP2, and TP3 and are shown in Figures 17,18 , and 19 . The no-go zone that was created to test that portion of the software was

Table 9. Mission planning with the graphical user interface of the mission planning software was very simple. Planning the new paths and modifying existing paths can be easily accomplished well within the goal of $30 \mathrm{~min}$.

\begin{tabular}{ccl}
$\begin{array}{c}\text { Path } \\
\text { Number }\end{array}$ & $\begin{array}{c}\text { Time to Construct } \\
\text { Path (min) }\end{array}$ & \multicolumn{1}{c}{ Notes } \\
\hline TP1 & 7 & $\begin{array}{l}\text { Simple 5-point path with one smooth fly-by and one pause point. } \\
\text { Requires the SGTV to transit from the start point to the end point and } \\
\text { then back-track along the same path. }\end{array}$ \\
TP2 & 18 & $\begin{array}{l}\text { More difficult path with } 10 \text { points, } 3 \text { smooth fly-bys, and } 2 \text { pause } \\
\text { points. This is a loop path, but does require the SGTV to backtrack a } \\
\text { portion of the path near the start/end point. } \\
\text { Complicated path with } 13 \text { points, } 6 \text { smooth fly-bys, and } 2 \text { pause points. } \\
\text { This is a loop path, but does require the SGTV to backtrack a portion of } \\
\text { the path near the start/end point. }\end{array}$ \\
\hline
\end{tabular}

loaded and used for the path planning. Table 9 shows the total time to construct each path. This included the time to design the path, draw the path on the computer screen, argue about practical and aesthetic virtues of each path, make any modifications, execute the collision detection prediction routine, and save the path to a data file for future recall.

\section{Display and command graphical user interface (GUI)}

All of the functions on the VCCG display and command graphical user interface were tested and performed as specified. Figure 13 shows a screen capture of the graphical user interface of the VCCG used to control the SGTV and log the data. In addition to the specification requirements, an analog and digital display of the angular rate of turn of the SGTV were added to assist with the VCCG/SGTV command and control development.

\section{Communications and data logging}

The VCCG program contains all of the data communication interfaces required in the specification. Testing confirmed that the VCCG receives feedback data from the SGTV including speed, angular turn rate, heading, GPS position, and system health monitoring data.

Two additional functions were added to the interface to assist with data logging, interpretation, and capture:

1. The VCCG can log data for non-self-guided (teleoperated) operations. This allows the VCCG data logging capabilities to capture certain aspects of teleoperations, 
such as linear and angular velocity command input signals and SGTV response and time stamps. Unfortunately, off-track error is not available since the VCCG is not in control of the SGTV during teleoperations.

2. The VCCG can convert and save the binary data log file to an ASCII file for importing the data into a spreadsheet or other program for data visualization and analysis.

The specification stated that the update rate for the VCCG (cycle time for the computer to sample data) was to be $50 \mathrm{~ms}$. This update period was increased to $130-150 \mathrm{~ms}$. There are two reasons for this change. First, gathering the data and then writing it to the hard drive on the computer eats up processor time and slows down the VCCG. Less data in the data stream significantly speeds up operations. Second, it was determined that the amount of data gathered at $50 \mathrm{~ms}$ was excessive for the analytical needs and the size of the data files were becoming unmanageable.

\subsection{System Integration}

System integration was intended to investigate the interface between the SGTV and the other equipment that it would be working with during retrieval operations. Specifically, the two pieces of equipment of interest were the remote excavator or REMEX with the innovative end effector (IEE), and the cooperative telerobotic retrieval (CTR) gantry crane. Unfortunately, the REMEX was not available at the time the actual testing took place, and the CTR gantry crane was not operational, and thus could not participate in the test. However, some very limited integration was demonstrated during the LSFA exposition in August, 1995 at the INEEL. The results of that demonstration will be briefly discussed here.

\subsubsection{Analysis}

No data analysis was required. No formal data gathering was accomplished due to the reasons previously stated.

\subsubsection{Results and Evaluation}

4.4.2.1 REMEX Integration. The LSFA exposition procedure required the SGTV to remotely navigate to a loading position and open the WTC lid to enable the REMEX with the innovative end effector to pickup an ITM, perform a dig in the simulated waste pit, and then place the loaded ITM into the SGTV. The SGTV then closed the lid and proceeded to a point underneath the CTR gantry crane where it would receive another ITM from the CTR 5-ton hoist.

The procedure for loading and unloading ITMs from the SGTV using the REMEX is very similar to what was used in FY-94 with the previous incarnation of the waste transport vehicle and innovative end effector. The primary difference in the configurations is that the innovative end effector for FY-95 was a backhoe configuration as opposed to the FY-94 front shovel configuration. The WTC lid was modified by moving the hinge to the end of the box to accommodate the backhoe configuration (See Figure 21). 

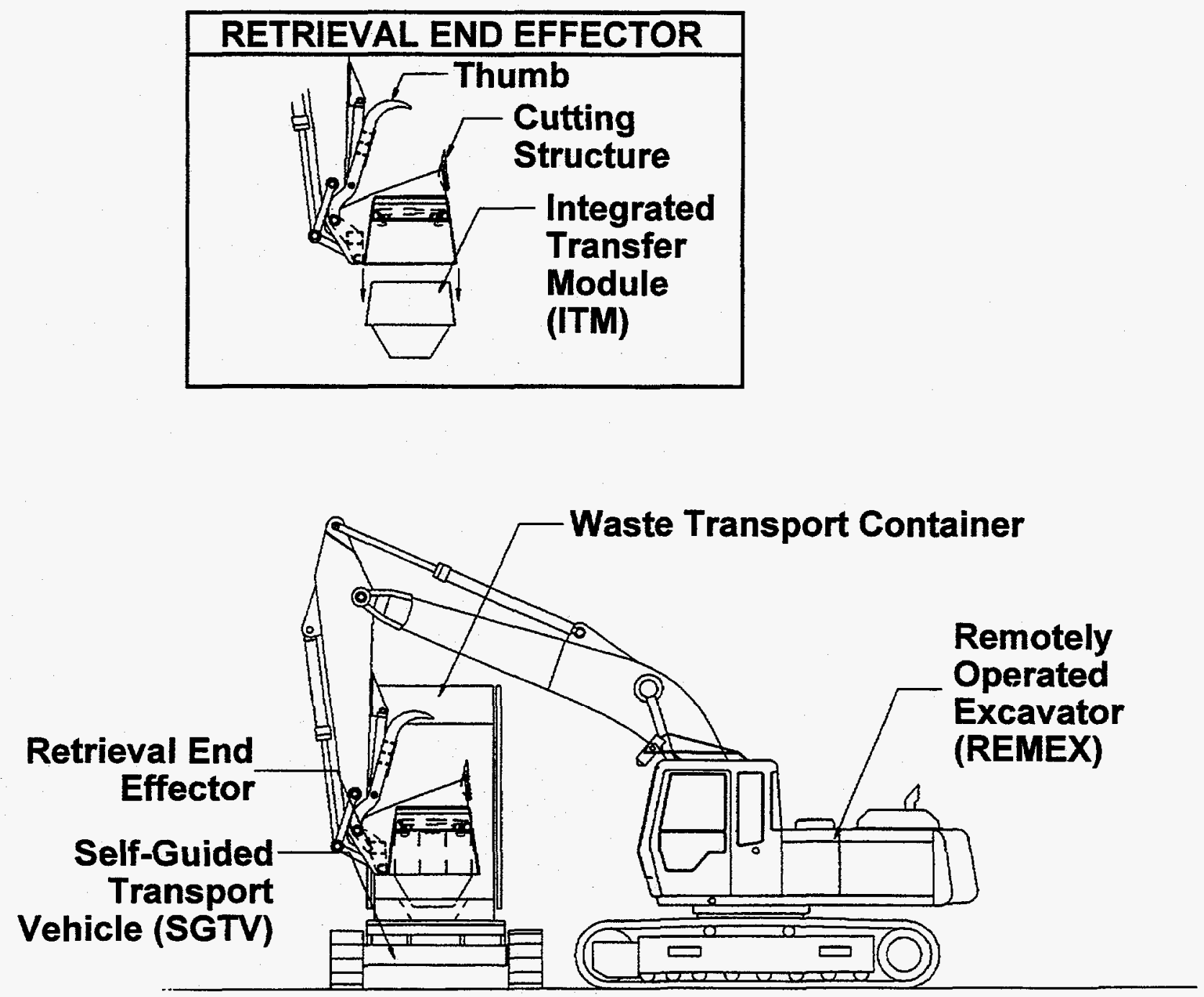

Figure 21. The waste transport container was modified from the FY-94 configuration to open at the end of the box to allow clearance for the REMEX stick in the backhoe configuration.

No significant issues were identified regarding picking up an ITM from the SGTV or placing an ITM into the WTC. The only operational difference is that the REMEX must be significantly closer to the SGTV to accomplish the task due to the change from the front shovel configuration. The process and techniques are very similar to those used during the FY-94 demonstration. The biggest issue associated with this interface is the lack of stereo vision for the REMEX operator. The task of picking up and placing the ITMs on the SGTV with limited two-dimensional camera views is very difficult. This could be addressed with the use of stereo vision cameras or increasing the number of views available (especially right angle views) to the REMEX operator. The operator did note that the backhoe configuration does offer a better view of the end effector than the front shovel configuration.

The IEE system and SGTV were developed in close coordination with one another to ensure a systems design approach that maximizes the effectiveness of the combined 
technologies. This approach provided for smooth and easy integration between all three pieces of equipment, the SGTV, IEE, and REMEX.

4.4.2.3 CTR Gantry Crane Integration. The SGTV is designed to navigate and dock at a location underneath the CTR gantry crane that will allow the crane to transfer waste to the WTC and to pick up and use ITMs delivered by the SGTV. The CTR gantry crane should be capable of delivering loaded ITMs, waste boxes up to $4 \times 4 \times 8 \mathrm{ft}$, waste drums, and oversized objects up to $4 \times 4 \times 8 \mathrm{ft}$ in size to the SGTV for placement in the WTC. The cooperative retrieval hoist adapter was specifically designed to provide the CTR 5-ton hoist the capability to robotically manipulate the ITMs. Other handling devices, such as drum grapples, are used to manipulate other waste forms.

During the LSFA exposition, the CTR only managed to provide one waste transfer to the SGTV. A single 55-gal drum was retrieved from the simulated waste pit with the drum grapple and successfully placed inside the WTC next to an ITM that had been previously loaded by the REMEX. Technical problems with the CTR gantry prevented any other attempts to load waste into the SGTV. The CTR gantry crane was not able to pick up the cooperative retrieval hoist adapter to test the capability to unload and load ITMs onto the SGTV.

Placing the drum in the WTC with the CTR gantry was performed telerobotically. The stereo vision system on the CTR along with a large variety of auxiliary camera views made the task significantly easier (in terms of depth perception for the operation) than the task of loading the ITMs with the REMEX. Based on this one observation, reviews of the CTR equipment design, and detailed discussions with the CTR design team, there appear to be no significant problems with the integration of the SGTV and the CTR gantry crane.

\subsubsection{Discussion/Conclusion}

The IEE system and SGTV were developed in close coordination with one another to ensure a systems design approach that maximizes the effectiveness of the combined technologies. This approach provided for smooth and easy integration between all three pieces of equipment, the SGTV, IEE, and REMEX. The flexible design of the SGTV and the adaptability of the CTR gantry crane provide for easy integration of the technologies for effective and efficient robotic waste retrieval operations.

\subsection{Precision and Accuracy of Navigation}

Data to support calculation of the precision and accuracy of navigation were obtained by determining the difference between the actual location of the vehicle and the desired location of the vehicle. The VCCG computer automatically logs the off-track error as determined by the GPS system and records the data for later recall and display. This section presents the data gathered from 30 self-guided test runs and several teleoperated runs for comparison.

\subsubsection{Analysis}

The primary statistical analysis used to determine the precision and accuracy of navigation was to determine the mean, standard deviation, and bias of the measurements with respect to the 
off-track error reported by the VCCG. Calculations for determining the mean, and precision and accuracy are as follows:

Mean: The mean or average off-track error was calculated two ways. First, the mean of the deviations was calculated by taking the absolute value of the off-track error as follows:

$\bar{X}=\frac{\sum_{i=1}^{n}\left|X_{i}\right|}{n}$

where

$$
\begin{array}{ll}
X_{i} & =\text { the off-track error measured at each cycle } \\
\bar{X} \quad & =\text { the mean off-track error } \\
n \quad & =\text { the total number of cycles completed per test run. }
\end{array}
$$

This value then represents the average offset distance maintained by the SGTV from the ideal path. Second, the absolute mean was calculated without considering the absolute value of the off-track error as follows:

$$
\bar{Y}=\frac{\sum_{i=1}^{n} Y_{i}}{n}
$$

where

$$
\begin{array}{lll}
Y_{i} & = & \text { the off-track error measured at each cycle } \\
\bar{Y} & =\quad \text { the absolute mean off-track error } \\
n & =\quad \text { the total number of cycles completed per test run. }
\end{array}
$$

This value represents the location of the average path from the ideal path. As will be noted later, it turns out that this value is the accuracy of SGTV tracking.

Precision: Two methods were used to calculate the precision. The first is a calculation of the average deviation of the observations from each other or the standard deviation around the average path. For this calculation, the absolute mean is used and the equation is as follows:

$$
S=\sqrt{\frac{\sum_{i=1}^{n}\left(Y_{i}-\bar{Y}\right)^{2}}{n-1}}
$$

where 


$$
\begin{array}{lll}
Y_{i} & = & \text { the off-track error measured at each cycle } \\
\bar{Y} & = & \text { the absolute mean off-track error } \\
\mathrm{S} & = & \text { the standard deviation (precision) } \\
n & =\quad \text { the number of observations. }
\end{array}
$$

The other method of determining the precision is to calculate the Mean Absolute Deviation (MAD), which yields the average absolute deviation from the average path. For this calculation, the absolute mean is also used and the equation is as follows:

$$
M A D=\frac{\sum_{i=1}^{n}\left|Y_{i}-Y\right|}{n}
$$

where

$$
\begin{array}{lll}
Y_{i} & =\quad \text { the off-track error measured at each cycle } \\
\bar{Y} & =\quad \text { the absolute mean off-track error } \\
M A D & =\text { the mean absolute deviation } \\
n & =\quad \text { the number of observations. }
\end{array}
$$

Accuracy (Bias): The accuracy is the average deviation from the value expected. The formula for calculating the accuracy is as follows:

$$
\text { Bias }=\frac{\sum_{i=1}^{n}\left(\mu_{0}-Y_{i}\right)}{n}
$$

where

$$
\begin{array}{ll}
Y_{i} & =\text { the off-track error measured at each cycle } \\
\mu_{o} \quad= & \text { the target value } \\
n \quad=\quad \text { the number of observations. }
\end{array}
$$

Notice that the bias is the same as the value of $\bar{Y}$ previously defined as the absolute mean. This is because the target value for the off-track error is zero, thus reducing the equation to the same as that for the absolute mean. 


\subsubsection{Results and Evaluation}

Appendix A contains the data logs and screen captures for each of the self-guided test runs performed for the precision and accuracy testing. Also included are the kinematic simulation runs for each of the test paths as a baseline for comparison.

Each of the data logs contains six graphs. The following is an explanation of each of the graphs:

- Time (s) vs Cycle: Data is sampled on each VCCG computer cycle. Each cycle takes approximately $150 \mathrm{~ms}$. This graph relates the data sampling to real time in seconds. All other graphs are based on cycle time.

- Off-Track Error (ft) vs Cycle: This is the actual off-track error calculated by the VCCG. It represents the difference between the actual location of the SGTV as reported by the GPS and the desired location as computed by the VCCG.

- Linear Velocity Command (ft/s) vs Cycle: This is the linear velocity command signal generated by the VCCG and transmitted to the SGTV. It is compared to the actual linear velocity to determine proper vehicle response to the command signal.

- Angular Velocity Command (deg/s) vs Cycle: This is the angular velocity command signal generated by the VCCG and transmitted to the SGTV. It is compared to the actual angular velocity to determine proper vehicle response to the command signal.

- Linear Velocity Actual ( $\mathrm{ft} / \mathrm{s}$ ) vs Cycle: This is the actual linear velocity response of the SGTV. It is compared to the linear velocity command signal to determine if the SGTV is responding appropriately to the VCCG commands.

- Angular Velocity Actual (deg/s) vs Cycle: This is the actual angular velocity response of the SGTV. It is compared to the angular velocity command signal to determine if the SGTV is responding appropriately to the VCCG commands.

The screen capture was obtained directly from the VCCG at the conclusion of each test run. The actual path of the SGTV is superimposed on top of the planned path. Note that some of the turns (especially $90^{\circ}$ turns) are smooth fly-by turns and are not intended to track on the planned path line, but are instead gradual turns following an arc-like path. Figures 17, 18, and 19 provide details of the path designs. Table 10 provides a listing and summary of each graph with its associated screen capture. The data code for each test run is noted at the bottom of each data $\log$ and screen capture. Graphs are located in Appendix A.

4.5.2.1 Statistical Analysis of Off-Track Error. The spreadsheet program, EXCEL, was used as the calculation tool to obtain the statistical results. Regression analyses was also performed on the results for each path to determine if trends existed in the average performance.

4.5.2.2 Evaluation of Average Off-Track Error. The mean and absolute mean off-track error was determined for each test run for each of the three paths, and are presented in Tables 11, 12 , and 13 and Figures 22, 23, 24 and 25 below. Regression analysis of the average off-track 
error results show the trends in the test averages. The trends shown in the figures indicate that between each test and for each path, improvements made in the VCCG tracking control algorithm parameters did reduce the off-track error. The Path 1 trend data show significantly greater off-track error averages than Paths 2 and 3, thus indicating a possible improvement in tracking control after Path 1.

Table 10. Reference key for precision and accuracy test run data.

\begin{tabular}{|c|c|c|}
\hline $\begin{array}{c}\text { Test Trial } \\
\text { (data } \\
\text { code) }\end{array}$ & Description & Notes \\
\hline TP1_KIN & $\begin{array}{l}\text { Test Path } 1 \text { - } \\
\text { Kinematic Simulation }\end{array}$ & Kinematic simulation for full cycle (point 1 to point 5 and return) \\
\hline TP1_R1 & Test Path 1 - Run 1 & \\
\hline TP1_R2 & Test Path 1 - Run 2 & \\
\hline TP1_R3 & Test Path 1 - Run 3 & 1 telemetry error \\
\hline TP1_R4 & Test Path 1 - Run 4 & 1 telemetry error, 1 network error \\
\hline TP1_R5 & Test Path 1 - Run 5 & \\
\hline TP1_R6 & Test Path 1 - Run 6 & 5 network errors (all reset) account for $\sim 10 \mathrm{~s}$ \\
\hline TP1_R7 & Test Path 1 - Run 7 & 1 network error - Caused large off-track error between point 2 and 3 . \\
\hline TP1_R8 & Test Path 1 - Run 8 & \\
\hline TP1_R9 & Test Path 1 - Run 9 & \\
\hline TP1_R10 & Test Path 1 - Run 10 & \\
\hline TP2_KIN & $\begin{array}{l}\text { Test Path } 2 \text { - } \\
\text { Kinematic Simulation }\end{array}$ & \\
\hline TP2_R1 & Test Path 2 - Run 1 & 1 network error \\
\hline TP2_R2 & Test Path 2 - Run 2 & \\
\hline TP2_R3 & Test Path 2 - Run 3 & 1 network error \\
\hline TP2_R4 & Test Path 2 - Run 4 & 1 telemetry error \\
\hline TP2_R5 & Test Path 2 - Run 5 & \\
\hline TP2_R6 & Test Path 2 - Run 6 & 1 telemetry error \\
\hline TP2_R7 & Test Path 2 - Run 7 & 1 network error. Compass error at pt 9 on way out caused off-track error. \\
\hline TP2_R8 & Test Path 2 - Run 8 & $\begin{array}{l}3 \text { telemetry errors. Big off-track error just after pt } 3 \text {. This is similar to other } \\
\text { runs. Some anomaly keeps interfering with the compass at this point. }\end{array}$ \\
\hline TP2_R9 & Test Path 2 - Run 9 & 1 network error \\
\hline TP2_R10 & Test Path 2 - Run 10 & $\begin{array}{l}2 \text { telemetry errors. Repeat of off-track error at point } 3 \text {. May be caused by } \\
\text { welding on the CTR. }\end{array}$ \\
\hline TP3_KIN & $\begin{array}{l}\text { Test Path } 3 \text { - } \\
\text { Kinematic Simulation }\end{array}$ & \\
\hline TP3_R1 & Test Path 3 - Run 1 & Unknown e-stop after pt 2 (probably OAS). Large compass error at pt 10. \\
\hline TP3_R2 & Test Path 3 - Run 2 & 1 telemetry error. Loss of data between pts 9 and 10 . Could be GPS related. \\
\hline
\end{tabular}


Table 10 (continued).

\begin{tabular}{|c|c|c|}
\hline $\begin{array}{l}\text { Test Trial } \\
\text { (data code) }\end{array}$ & Description & Notes \\
\hline TP3_R3 & Test Path 3 - Run 3 & 1 telemetry error \\
\hline TP3_R4 & Test Path 3 - Run 4 & 2 telemetry errors \\
\hline TP3_R5 & Test Path 3 - Run 5 & 1 telemetry 1 OAS error \\
\hline TP3_R6 & Test Path 3 - Run 6 & 2 network and 7 GPS errors (satellite coverage was marginal) \\
\hline TP3_R7 & Test Path 3 - Run 7 & 1 network error (required reboot) \\
\hline TP3_R8 & Test Path 3 - Run 8 & $\begin{array}{l}1 \text { network error (repeating at same location as in other test runs). } \\
\text { Severe heading error between pts } 9 \text { and } 10 \text {. Several other heading } \\
\text { errors. Worse run yet. Suspect electromagnetic interference. }\end{array}$ \\
\hline TP3_R9 & Test Path 3 - Run 9 & $\begin{array}{l}1 \text { network } 1 \text { telemetry error. Good smooth run. Did not experience } \\
\text { same problems as previous run }\end{array}$ \\
\hline TP3_R10 & Test Path 3 - Run 10 & 2 network errors ( 1 required reboot) \\
\hline
\end{tabular}

Table 11. Statistical analysis for test path 1 data.

Off-Track Error - Path 1

\begin{tabular}{|c|c|c|c|c|}
\hline & $\begin{array}{c}\bar{X} \\
\text { (Mean Off-Track } \\
\text { Error) }\end{array}$ & $\begin{array}{c}\bar{Y} \\
\text { (Absolute Mean Off- } \\
\text { Track Error, also } \\
\text { Accuracy or Bias) }\end{array}$ & $\begin{array}{c}S \\
\text { (Precision - } \\
\text { Standard Deviation) }\end{array}$ & $\begin{array}{c}M A D \\
\text { (Precision - Mean } \\
\text { Absolute Deviation) }\end{array}$ \\
\hline TP1-KIN & 0.087 & -0.087 & 0.200 & 0.124 \\
\hline TP1-R1 & 0.637 & -0.430 & 0.914 & 0.623 \\
\hline TP1-R2 & 0.499 & -0.274 & 0.749 & 0.514 \\
\hline TP1-R3 & 0.618 & -0.482 & 0.879 & 0.572 \\
\hline TP1-R4 & 0.669 & -0.469 & 0.748 & 0.562 \\
\hline TP1-R5 & 0.649 & -0.518 & 0.853 & 0.553 \\
\hline TP1-R6 & 0.487 & -0.213 & 0.858 & 0.495 \\
\hline TP1-R7 & 0.859 & -0.603 & 1.059 & 0.853 \\
\hline TP1-R8 & 0.469 & -0.177 & 0.683 & 0.478 \\
\hline TP1-R9 & 0.613 & -0.399 & 0.940 & 0.626 \\
\hline TP1-R10 & 0.515 & -0.168 & 0.735 & 0.522 \\
\hline $\begin{array}{l}\text { Average of } \\
\text { tests R1-R10 }\end{array}$ & 0.602 & -.373 & 0.842 & 0.580 \\
\hline
\end{tabular}


Table 12. Statistical analysis for test path 2 data.

\begin{tabular}{lcccc}
\hline \multicolumn{5}{c}{ Off-Track Error - Path 2 } \\
\hline & $\begin{array}{c}\bar{X} \\
\text { (Mean Off-Track } \\
\text { Error) }\end{array}$ & $\begin{array}{c}\bar{Y} \\
\text { (Absolute Mean Off- } \\
\text { Track Error, also } \\
\text { Accuracy or Bias) }\end{array}$ & $\begin{array}{c}S \\
\text { (Precision } \\
\text { Standard } \\
\text { Deviation) }\end{array}$ & $\begin{array}{c}\text { MAD } \\
\text { (Precision - Mean } \\
\text { Absolute Deviation) }\end{array}$ \\
\hline TP2-KIN & 0.507 & -0.100 & 0.873 & 0.536 \\
TP2-R1 & 0.567 & -0.209 & 0.879 & 0.579 \\
TP2-R2 & 0.616 & -0.309 & 0.934 & 0.634 \\
TP2-R3 & 0.550 & -0.144 & 0.961 & 0.584 \\
TP2-R4 & 0.550 & -0.243 & 0.828 & 0.554 \\
TP2-R5 & 0.707 & -0.188 & 1.092 & 0.727 \\
TP2-R6 & 0.721 & -0.243 & 0.935 & 0.706 \\
TP2-R7 & 0.638 & -0.338 & 0.993 & 0.665 \\
TP2-R8 & 0.618 & -0.248 & 0.939 & 0.657 \\
TP2-R9 & 0.684 & -0.178 & 1.092 & 0.687 \\
TP2-R10 & 0.691 & -0.234 & 1.071 & 0.735 \\
Average of & 0.634 & -0.233 & 0.972 & 0.653 \\
tests R1-R10 & & & & \\
\hline
\end{tabular}

Table 13. Statistical analysis for test path 3 data.

\begin{tabular}{lcccc}
\hline \multicolumn{5}{c}{ Off-Track Error - Path 3 } \\
\hline & $\begin{array}{c}\bar{X} \\
\text { (Mean Off-Track } \\
\text { Error) }\end{array}$ & $\begin{array}{c}\bar{Y} \\
\text { (Absolute Mean Off- } \\
\text { Track Error, also } \\
\text { Accuracy or Bias) }\end{array}$ & $\begin{array}{c}\text { (Precision } \\
\text { Standard } \\
\text { Deviation) }\end{array}$ & $\begin{array}{c}\text { MAD } \\
\text { (Precision - Mean Absolute } \\
\text { Deviation) }\end{array}$ \\
\hline TP3-KIN & 0.660 & 0.091 & 0.945 & 0.679 \\
TP3-R1 & 0.606 & 0.020 & 0.818 & 0.605 \\
TP3-R2 & 0.820 & -0.248 & 1.041 & 0.823 \\
TP3-R3 & 0.660 & -0.039 & 0.889 & 0.661 \\
TP3-R4 & 0.750 & -0.027 & 0.978 & 0.753 \\
TP3-R5 & 0.662 & -0.241 & 0.899 & 0.679 \\
TP3-R6 & 0.776 & -0.198 & 0.982 & 0.782 \\
TP3-R7 & 0.703 & -0.044 & 0.974 & 0.702 \\
TP3-R8 & 0.803 & -0.032 & 1.086 & 0.808 \\
TP3-R9 & 0.663 & -0.157 & 0.832 & 0.653 \\
TP3-R10 & 0.647 & -0.214 & 0.841 & 0.643 \\
Average of & 0.709 & -0.118 & 0.934 & 0.711 \\
tests R1-R10 & & & & \\
\hline
\end{tabular}



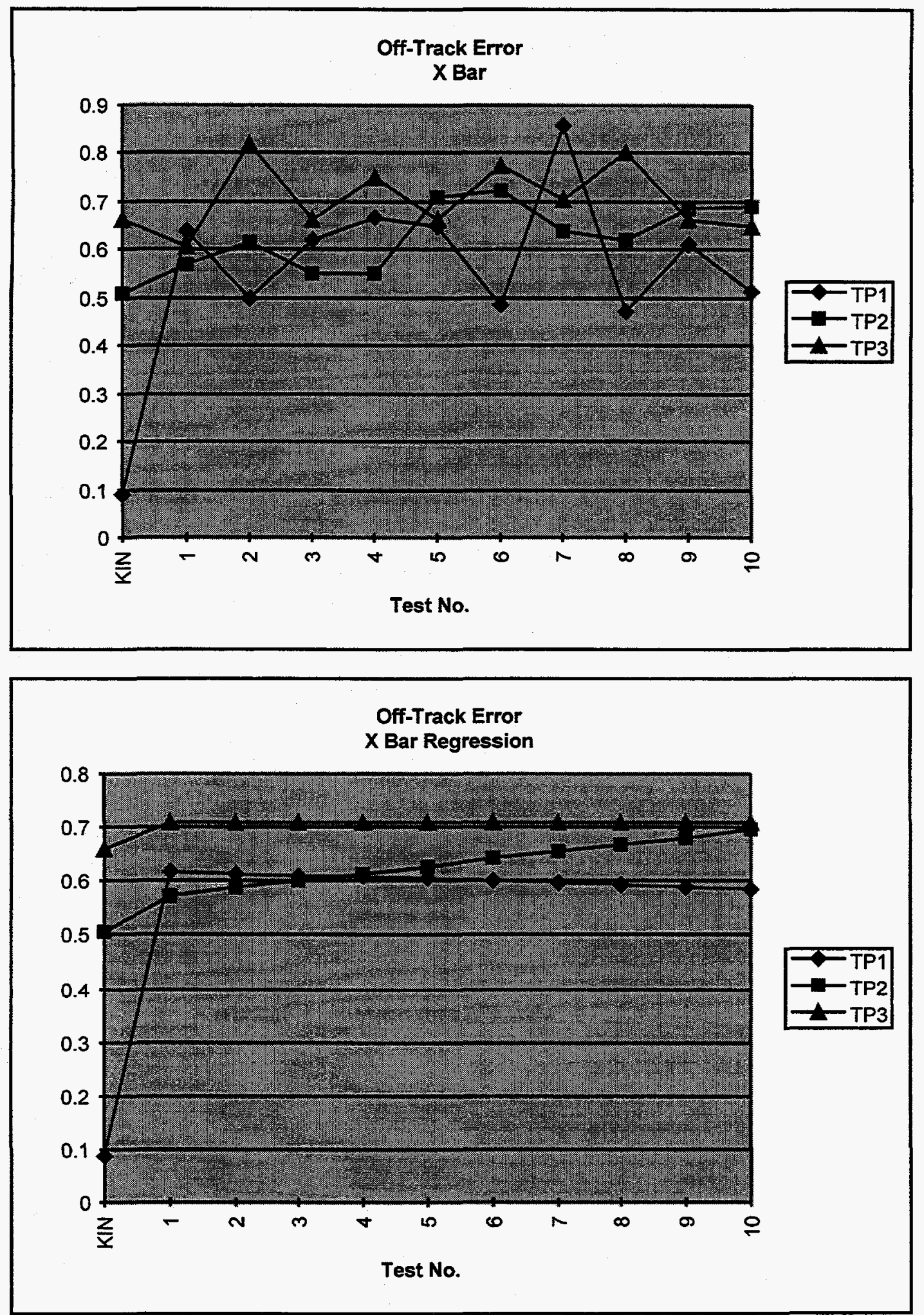

Figure 22. Data plot of the mean off-track error and regression curves of the mean off-track error. 

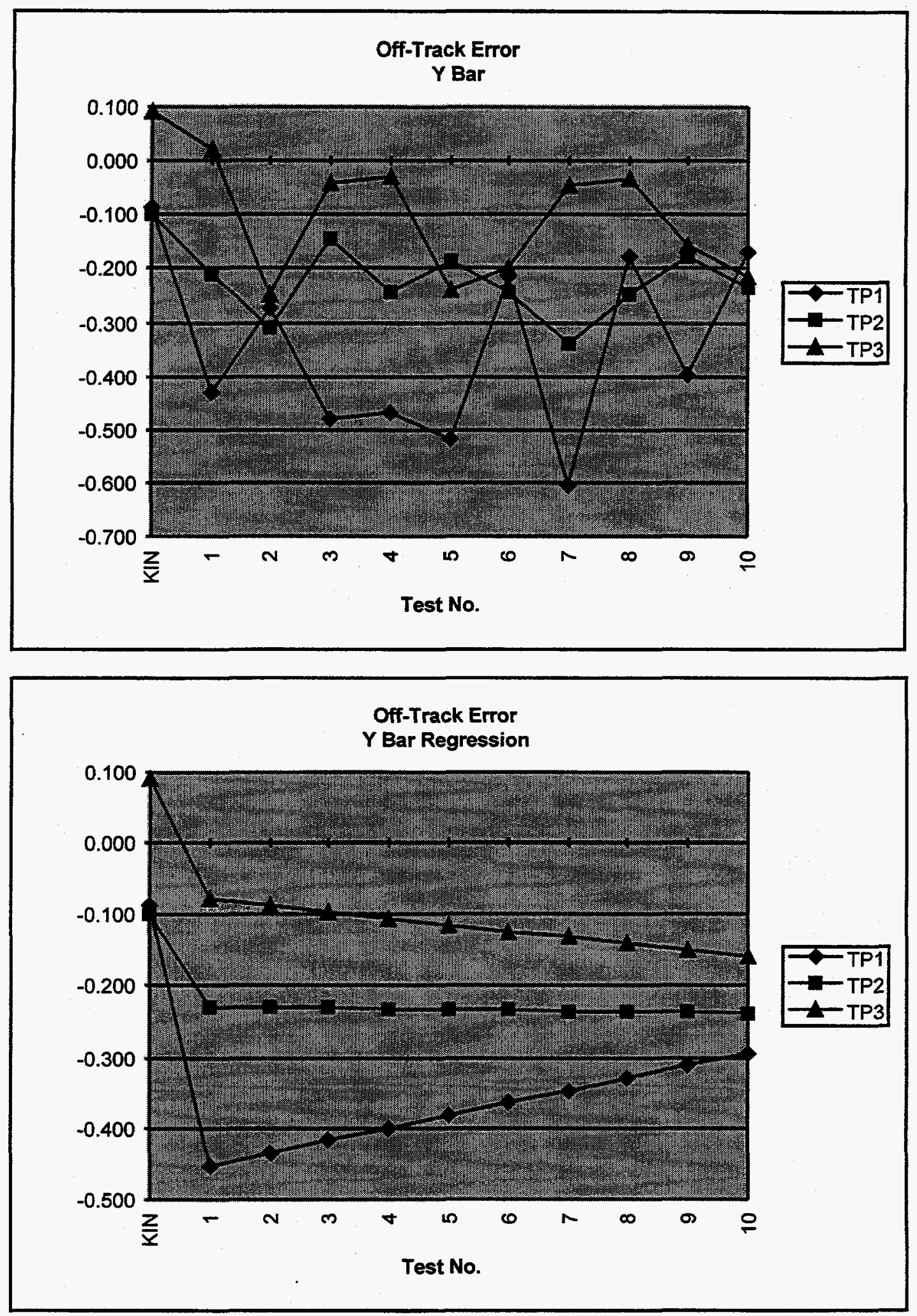

Figure 23. Data plot of the absolute mean off-track error (accuracy) and regression curves of the accuracy. 

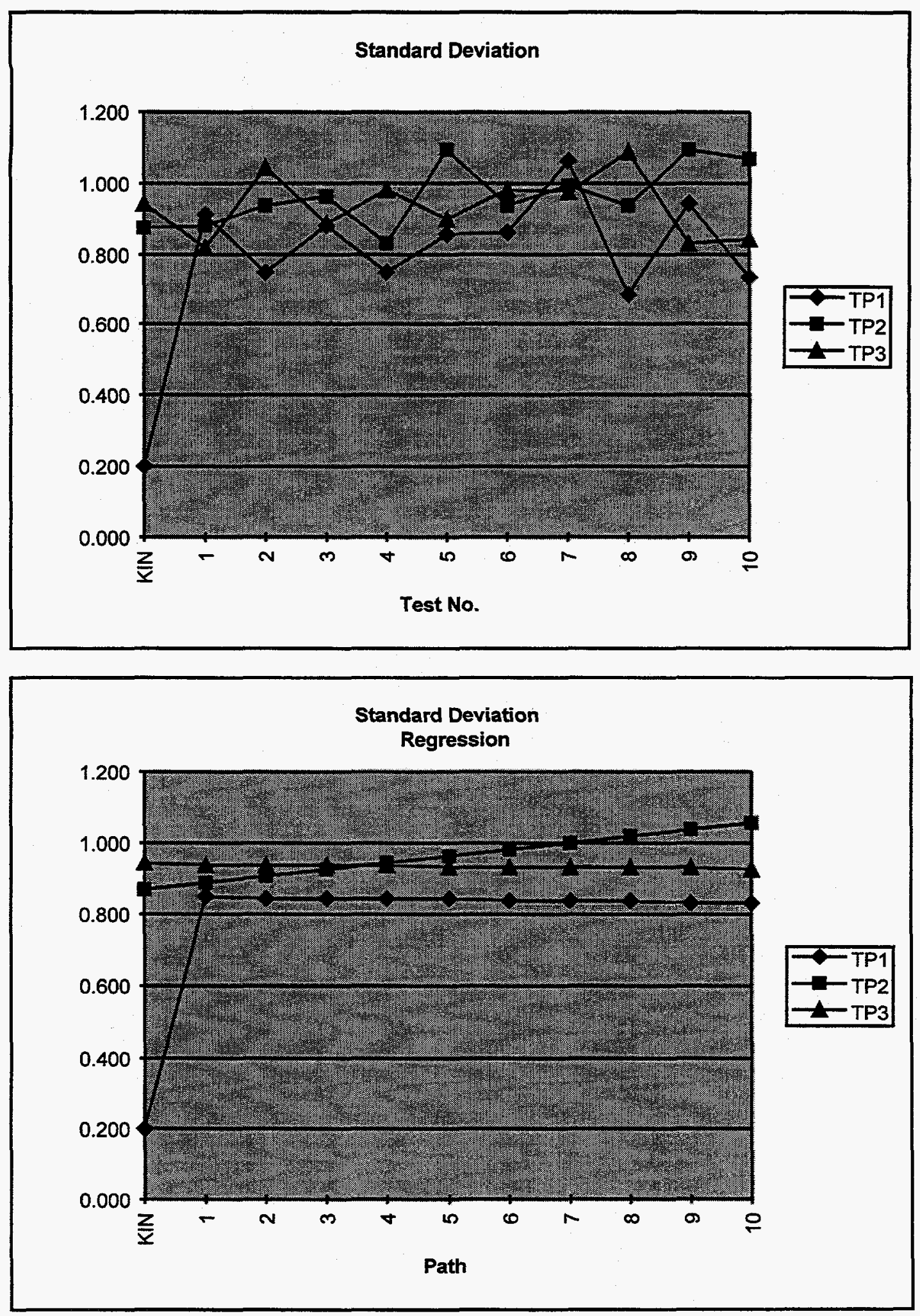

Figure 24. Data plot of the standard deviation precision calculations and associated regression analysis. 

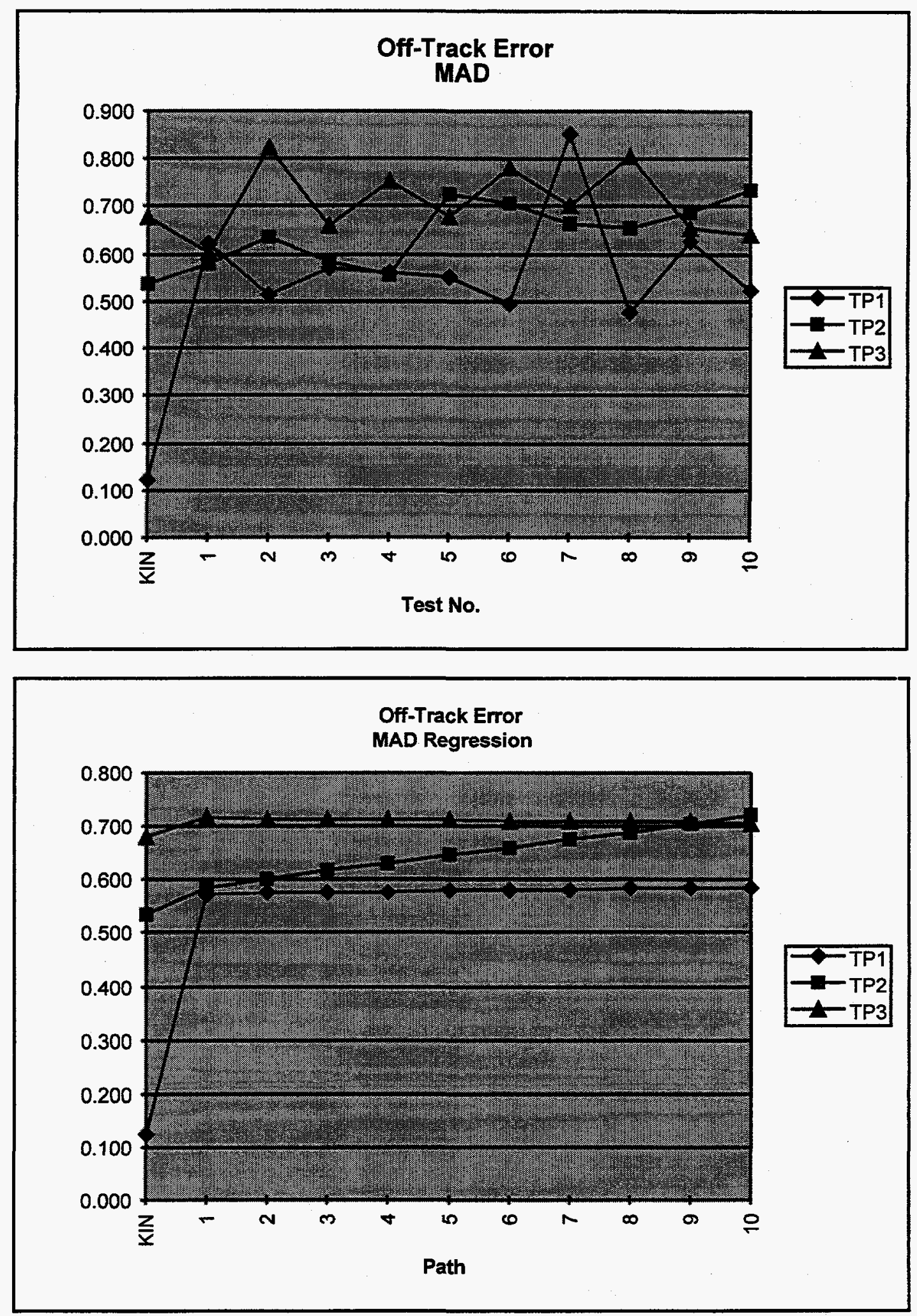

Figure 25. Data plot of the mean absolute deviation precision calculations and associated regression analysis. 


\subsubsection{Discussion/Conclusion}

4.5.3.1 Off-Track Errors. There are several very important things to understand about the off-track error to allow for proper interpretation of the data and reasonable conclusions about the precision and accuracy of SGTV navigation.

First, notice that the kinematic simulation shows an off-track error. In theory, the kinematic simulation is the perfect case, and shouldn't show any off-track error. However, the kinematic simulation does show an off-track error for the following reasons:

1. Off-track errors at "start" point, "pause" point, "pure flyby" point and "end" point.

When the vehicle reaches the circles of the above navigation points, the VCCG issues either a stop command or directs the SGTV to begin navigation along the next path segment. The SGTV then will track the next straight line (except at the "end" point). The radii for the "start" point, "pause" point and "pure flyby" point have a default setting of $2 \mathrm{ft}$, and the end point $1 \mathrm{ft}$. Since the kinematic simulation only has a time delay in the integration routine to determine the SGTV's position, and does not have vehicle dynamics to contend with, it will stop the SGTV just within the $2 \mathrm{ft}$ radius. As a result, an initial off-track error will exist, and will persist until the vehicle has traveled some distance to get back on track. As for the "end" point, the VCCG will stop the vehicle $1 \mathrm{ft}$ from the center of the "end" point circle. That means the position error at the completion of each run will be about $1 \mathrm{ft}$.

2. Off-track error along a straight line.

As was alluded to above, an off-track error also exists when the vehicle tracks a straight line in kinematic simulation. This is due to initial off-track error from the start, pause, or flyby point. The error is reduced over time via corrections in velocity and heading to eliminate the error.

Second, notice that in the kinematic simulation a large off-track error is evident during the "smooth flyby" sequences of the path tracking. The calculation of the off-track error at a "smooth flyby" point is different from straight-line tracking. In the "smooth flyby" case, the offtrack error is defined as the distance of the vehicle from an arc which has the two adjacent lines as its tangents. In straight line tracking the off-track error is the distance of the vehicle from the desired straight line.

During development, two different control algorithms were tested for use on the SGTV. The first one controls the vehicle by minimizing the off-track error defined above. The second method controls the distance of the vehicle from the next straight path and the heading from the desired heading simultaneously.

During the testing, it was found that the first controller caused excessive irregular vehicle motions, mainly due to time delays in the system. The second method proved to be more reliable and provide much smoother transitions between path segments. Therefore, the second method was implemented. However, this change was implemented late in the development, and the method of calculating the off-track was not changed to accommodate the control change. Therefore, a significantly larger off-track error is reported than actually exists. 
The same two conditions described above exist not only for the kinematic simulation, but for real-time control of the SGTV. The same control algorithms apply for both senarios. Therefore, due to system latency, these conditions may in many cases amplify the off-track errors of the real SGTV. At the very least, the off-track error of the SGTV should be compared not to zero, but to the ideal off-track error predicted by the kinematic simulation. However, this level of analysis is beyond the scope of this report and will be left to the reader to investigate. The best way to evaluate the precision and accuracy of navigation of the SGTV is to look at the path traces of the SGTV captured by the VCCG and (noting that the grid spacing on the plots is $10 \mathrm{ft}$ ) observe how well the vehicle tracks to the desired path and look at the smooth-flybys to determine if they were indeed smooth. (Note that the modified smooth flyby control algorithm results in parabolic shaped smooth flybys as opposed to the initial algorithm that was designed to provide circular shaped flybys). When you account for navigation errors induced by data network failures and the occasional GPS or compass error, the precision and accuracy of the SGTV is extremely good.

4.5.3.2 Statistical Analysis. As previously stated, the data used for the statistical analysis is not entirely reliable. The actual off-track errors are generally smaller than the raw data would support, and the precision and accuracy of navigation of the SGTV is much better than the data suggests. This is most evident if one chooses to overlook the shortcomings of the data and observe the actual path traces captured from the VCCG.

The mean off-track error regression analysis shows that TP3 had the highest mean offtrack error of the three test paths (Figure 22). This analysis also suggests performance on TP2 degraded between the first and last runs. This is well supported in the test notes by the increasing number and severity of network problems due to EMI problems that were occurring during this test sequence. The predicted off-track error for TP1_KIN was significantly different that the acutal mean off-track error. TP2_KIN and TP3_KIN were more closely related to the actual results.

The absolute mean off-track error regression analysis shows that overall tracking accuracy improved from the TP1 test series to the TP3 test series (Figure 23). This is consistent with path tracking algorithm improvements that were incorporated between these test runs. The increase in the complexity of the path traveled did not appear to increase the difficulty of achieving accurate tracking. The analysis also shows improved tracking for TP1 from test 1 to test 10 . However, the widely scattered data may not support this conclusion. The kinematic predictions for off-track accuracy were more optimistic than the actual results.

Figures 24 and 25 show the precision data and regression analysis for two methods of determining the precision (standard deviation and MAD). Both of these sets of graphs look very similar and only vary in the magnitude of the numbers depending on the method of calculation. The precision of tracking for TP2 again shows degraded performance over time as was discussed previously. The precision estimate for TP1_KN was significantly better than the actual precision for this test path. TP2_KIN and TP3_KIN were more closely related to the actual results.

An important thing to note in each of the regression curves shown in Figures 22, 23, 24 , and 25 is that the slope of the curves is very shallow or flat. This indicates that the performance of the SGTV for each of the test paths is highly repeatable. This provides a great deal of confidence that the SGTV tracking performance is very good, and only requires some system tweeking to achieve near zero off-track performance. 
4.5.3.3 Teleoperated Path Tracking. A series of tests were performed for comparing precision and accuracy of navigation between self-guided and teleoperated modes. Figures 26-33 are screen captures from a series of teleoperated tests (see Table 14 for a summary of each run). In these tests, an operator with many hours of experience in piloting the SGTV in teleoperated mode drove the vehicle around each of the three paths that were used for the self-guided tests. Operations were performed from the teleoperators control console with onboard video views for navigation. In addition to the on-board video the operator was provided with external video views, video target boards set up at end and intermediate points along the path, and the VCCG path tracking screen to assist with teleoperated navigation. Since the VCCG was not controlling the SGTV, an off-track error could not be calculated. However, it is obvious when comparing the screen captures of the self-guided test runs to the teleoperated test runs that the teleoperated runs have significantly greater off-track error than those under control of the VCCG.

Table 14. Reference key for the screen plots from the teleoperated precision and accuracy tests.

\begin{tabular}{ccc}
\hline $\begin{array}{c}\text { Test Trial } \\
\text { (data code) }\end{array}$ & \multicolumn{1}{c}{ Description } & Notes \\
\hline TP1TO_R1 & Test Path 1 - Teleoperated Run 1 & \\
TP1TO_R2 & Test Path 1 - Teleoperated Run 2 & \\
TP1TO_R4 & Test Path 1 - Teleoperated Run 4 & $\begin{array}{l}\text { Forgot to collect screen dump for run number } 3 \text { so } \\
\text { a fourth run was performed }\end{array}$ \\
& & \\
TP2TO_R1 & Test Path 2 - Teleoperated Run 1 & \\
TP2TO_R2 & Test Path 2 - Teleoperated Run 2 & \\
TP2TO_R3 & Test Path 2 - Teleoperated Run 3 & \\
TP3TO_R1 & Test Path 3 - Teleoperated Run 1 & \\
TP3TO_R2 & Test Path 3 - Teleoperated Run 2 & \\
\hline
\end{tabular}


[Y 01 Id]

$6 L$

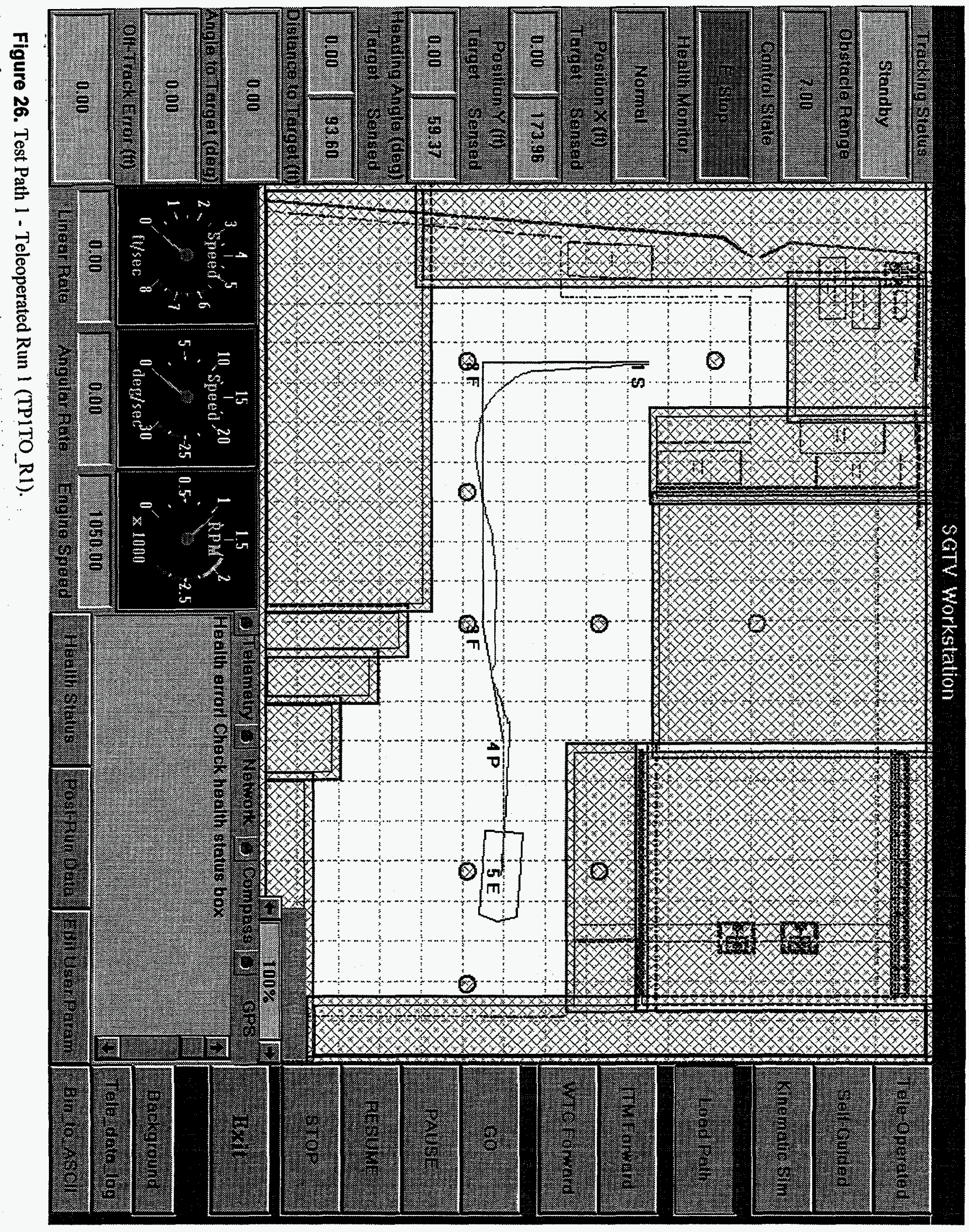




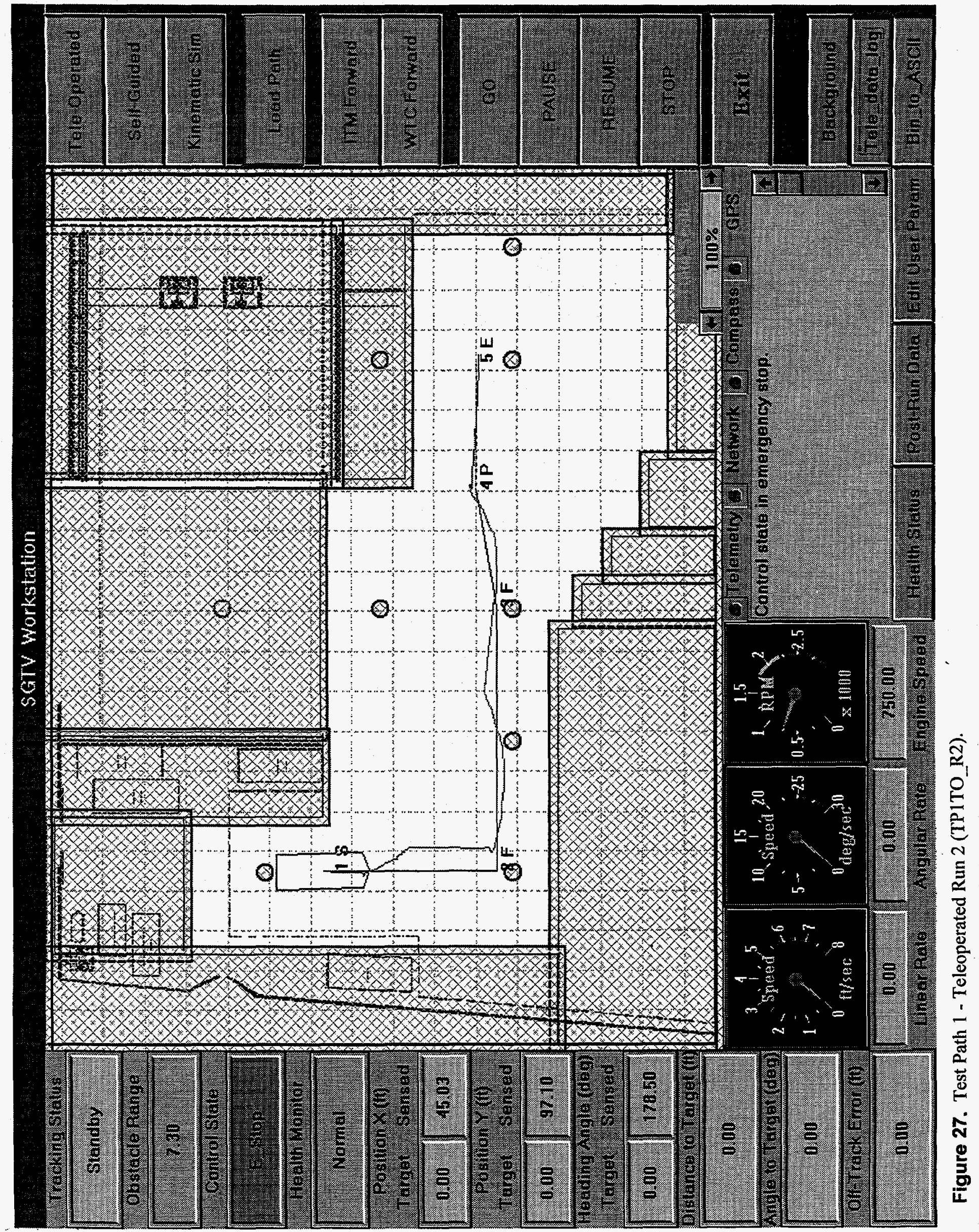


$\hbar y^{-} 0 \perp[d \perp$

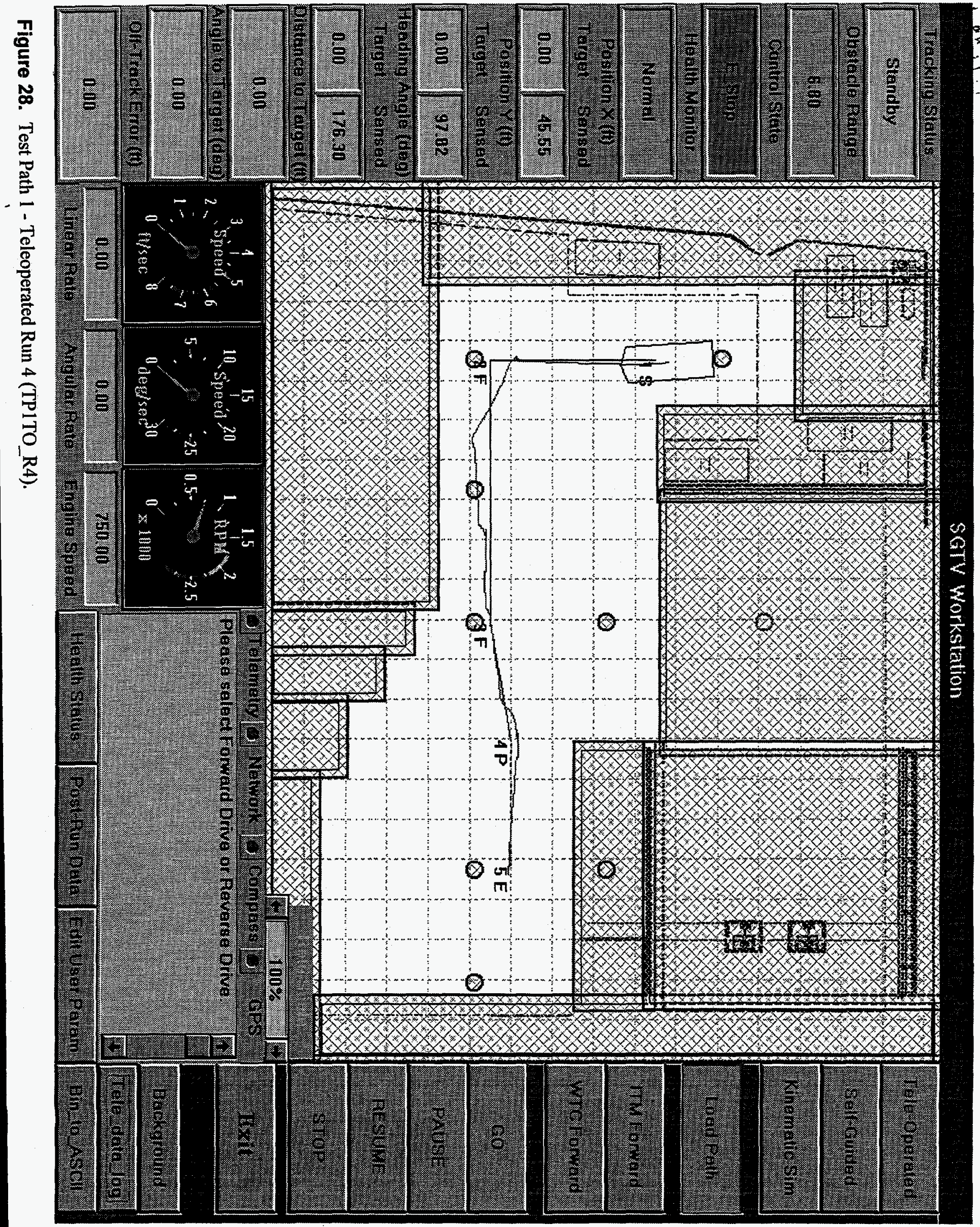




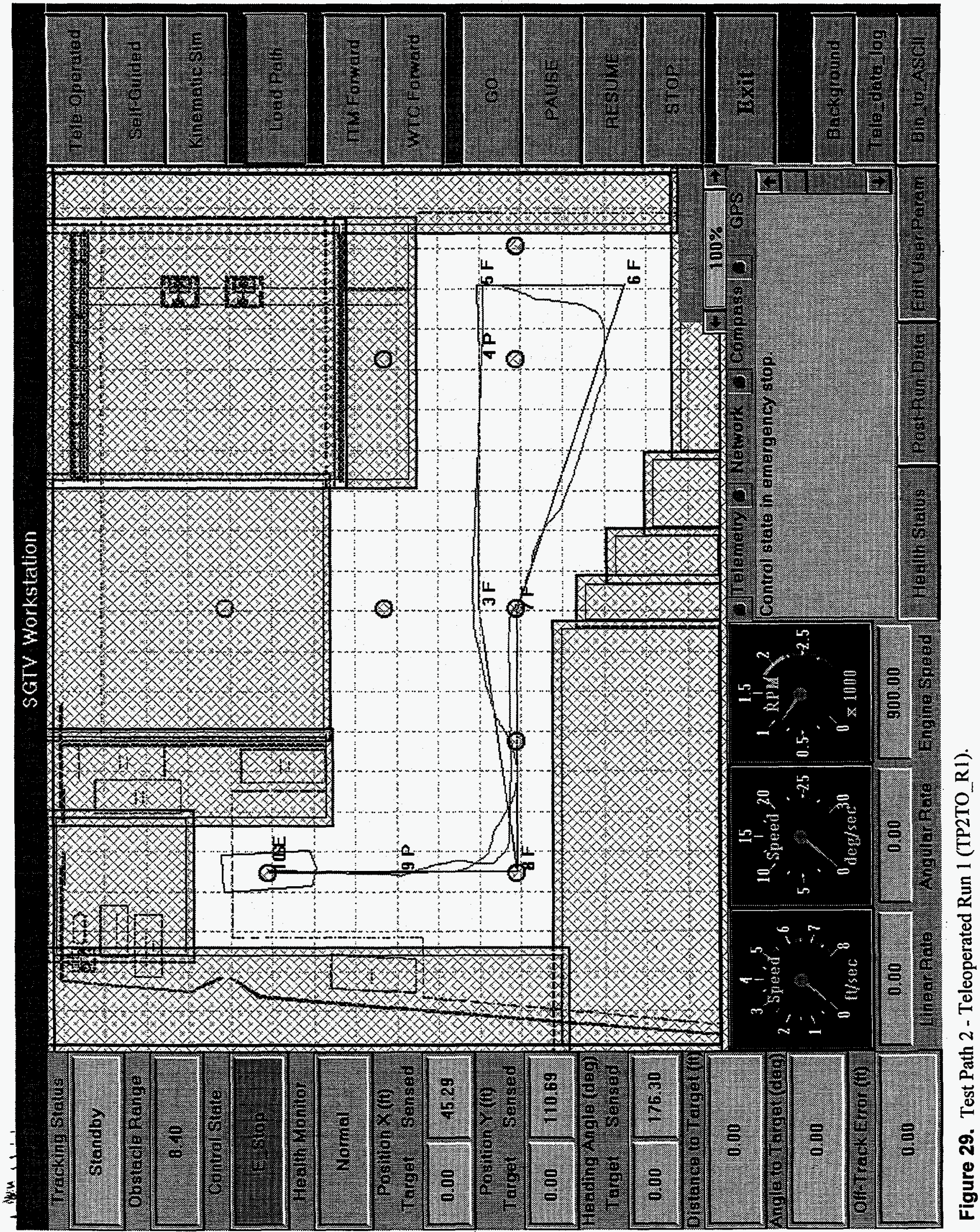



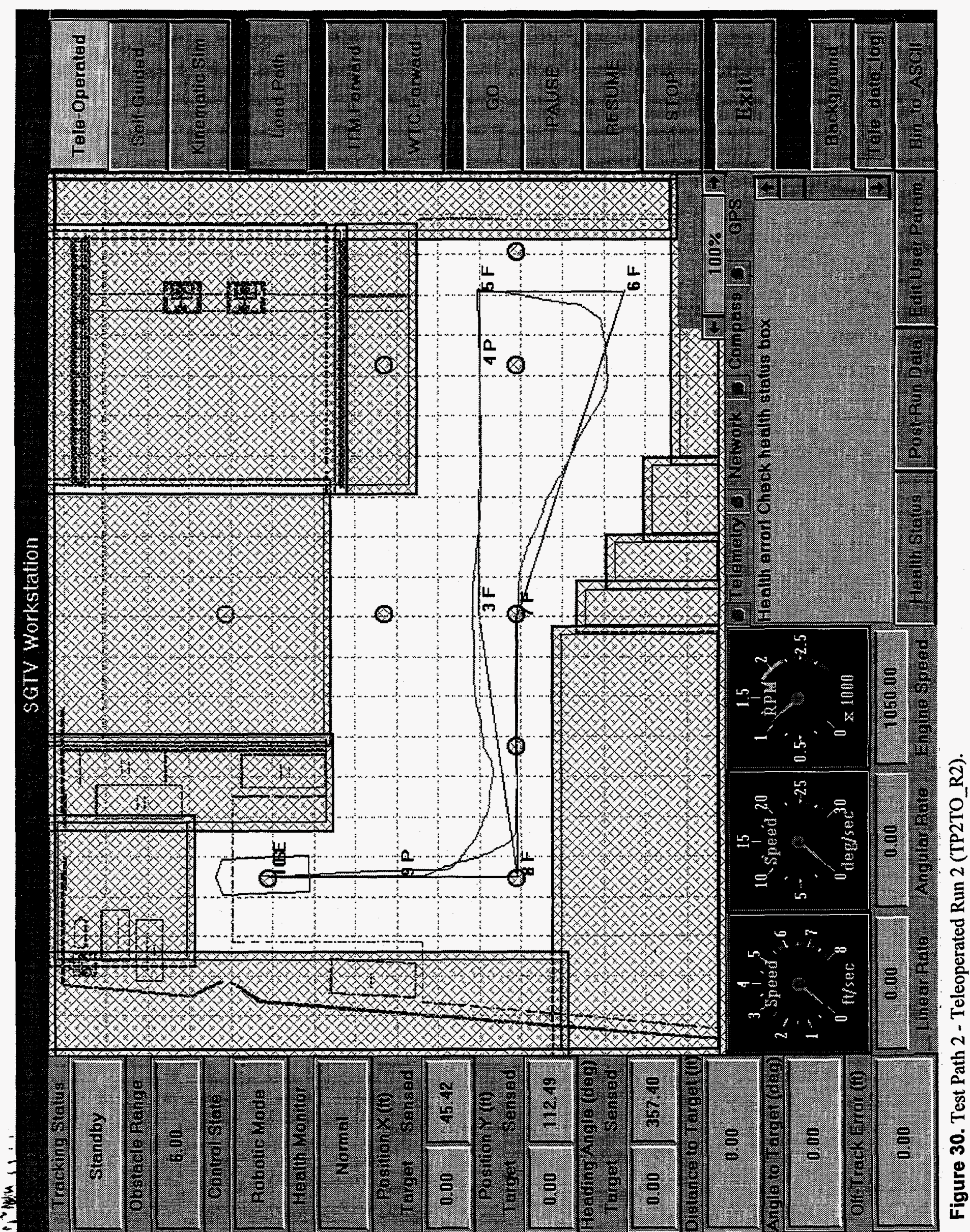


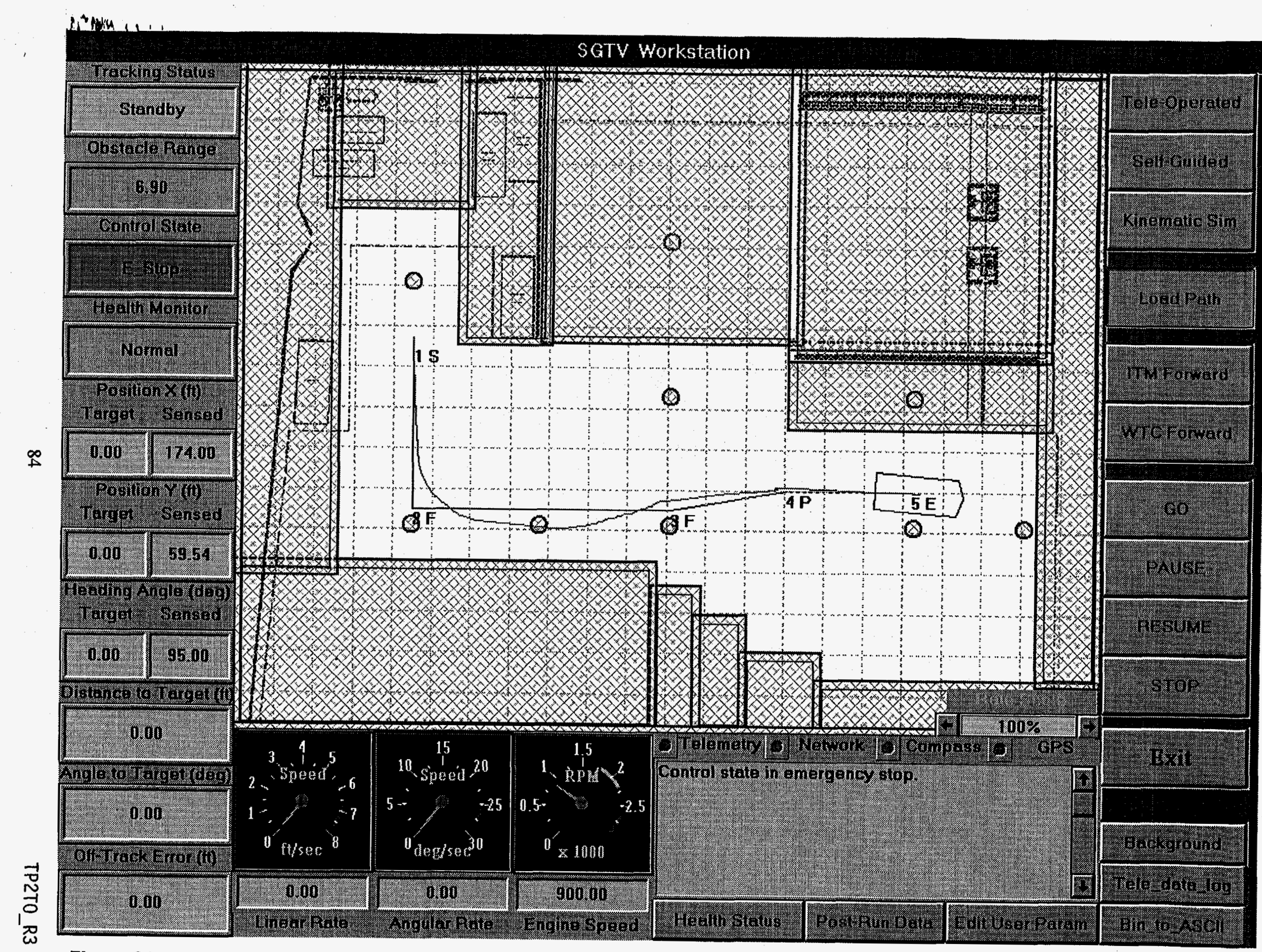

Figure 31. Test Path 2 - Teleoperated Run 3 (TP2TO_R3). 
โป $01 \varepsilon d \perp$

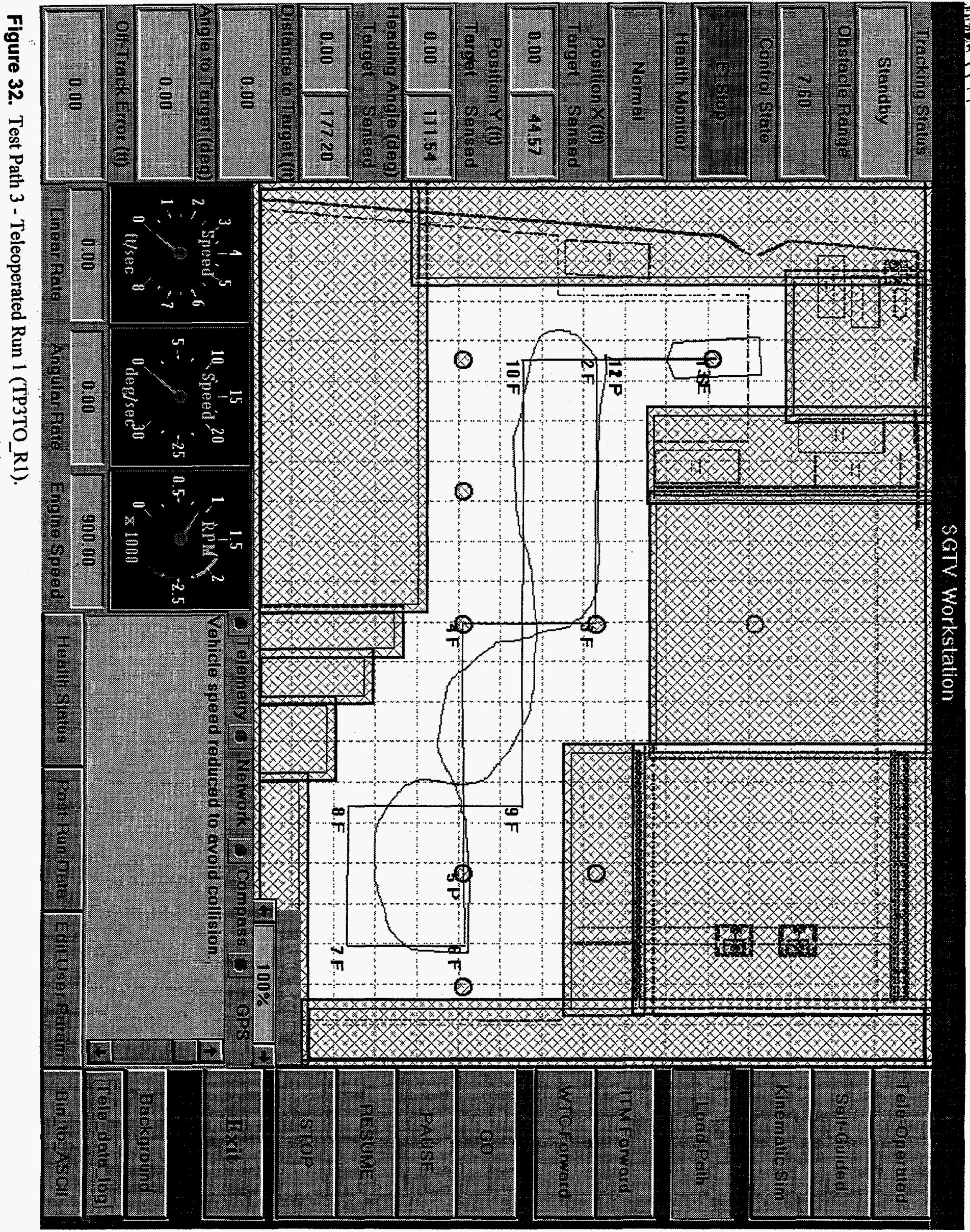


टУ $01 \varepsilon d \perp$

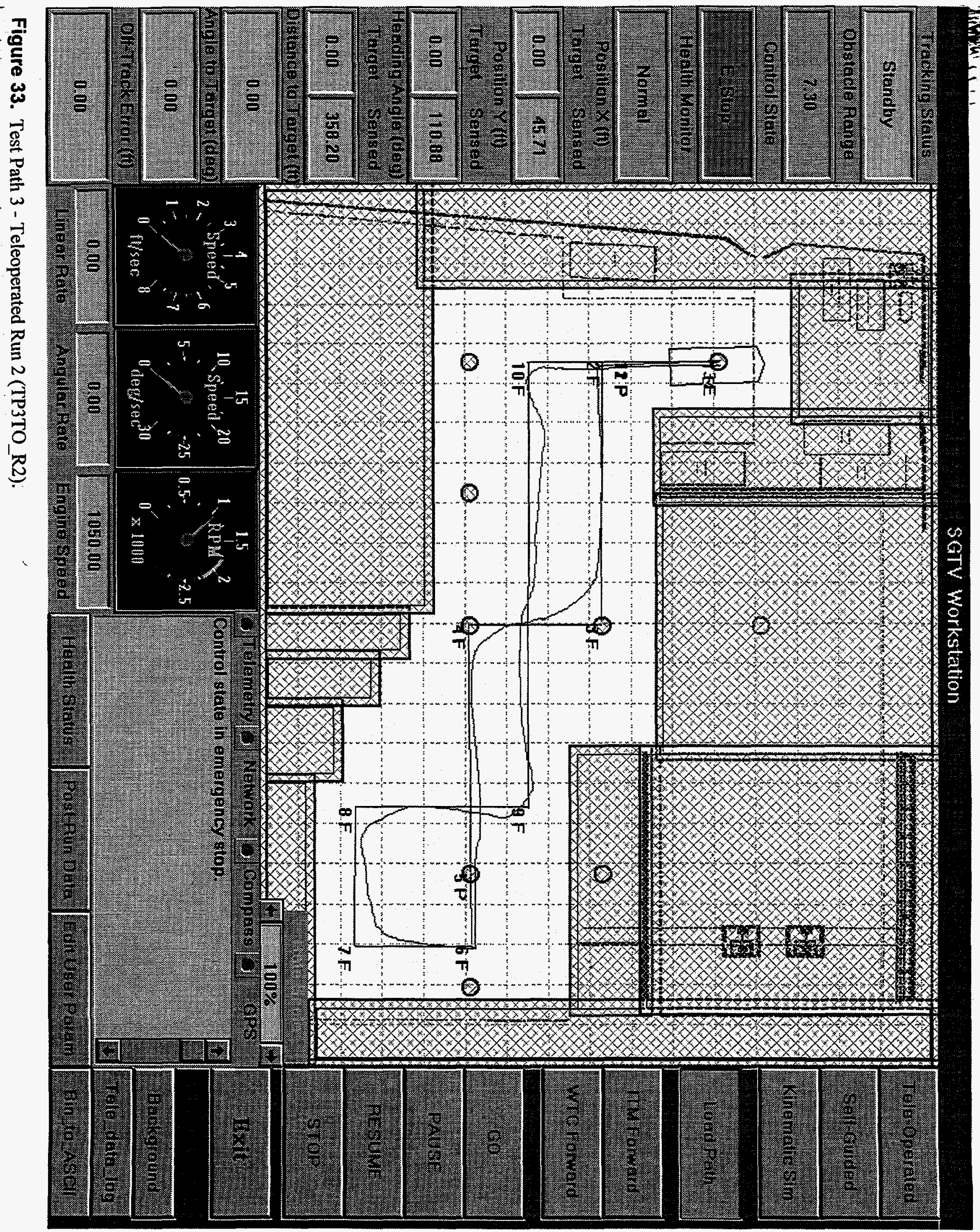




\subsection{SGTV Ultrasonic Obstacle Avoidance}

The ultrasonic obstacle avoidance system (OAS) was tested by quantitative means, which compare manual measurements with the on-board OAS ultrasonic sensors. The parameters measured that are of most interest in evaluating the OAS are the distance an obstacle is first detected, the distance an obstacle first enters the collision zone, and the distance when the emergency stop has been initiated by the obstacle avoidance system. The data was collected with a tape measure for the manual measurements and directly from the OAS ultrasonic sensor readings on the VCCG control station and documented in the log book and on data sheets.

\subsubsection{Analysis}

Data collected to document the performance of the obstacle avoidance is presented in the next section without analysis.

\subsubsection{Results and Evaluation}

The configuration and results of the OAS tests are given below in Tables 15-22.

Table 15. Results of OAS test series number 1.

\begin{tabular}{ccccc}
\hline Trial Number & Detection Zone $(\mathrm{ft})$ & Collision Zone $(\mathrm{ft})$ & \multicolumn{2}{c}{ E-Stop $(\mathrm{ft})$} \\
\hline 1 & OAS & OAS & Tape & OAS \\
\hline 2 & 22.5 & 19.5 & 3.8 & 3.0 \\
3 & 22.6 & 18.7 & 2.8 & 3.1 \\
& 24.1 & 18.2 & 3.4 & 2.7 \\
\hline
\end{tabular}

Trial configuration notes:

Mode - Self-guided

Direction of travel - Forward

SGTV Speed $-2.5 \mathrm{ft} / \mathrm{s}$

OAS Stopping Distance - $5 \mathrm{ft}$

Target - ITM face on to vehicle

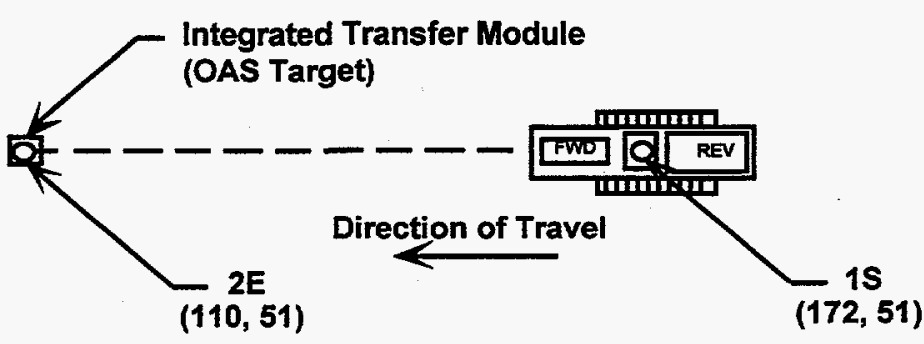

Notes: None 
Table 16. Results of OAS test series number 2. Notice that in this configuration, the OAS had a great deal of difficulty detecting the object and in fact hit it on two of the three trials.

\begin{tabular}{ccccc}
\hline Trial Number & Detection Zone (ft) & Collision Zone $(\mathrm{ft})$ & \multicolumn{2}{c}{ E-Stop $(\mathrm{ft})$} \\
\hline OAS & OAS & Tape & OAS \\
\hline 4 & Not Detected & Not Detected & na & Hit ITM \\
5 & Not Detected & Not Detected & na & Hit ITM \\
6 & Not Detected & Not Detected & 1.6 & 2.0 \\
\hline
\end{tabular}

Trial configuration notes:

Mode - Self-guided

Direction of travel - Forward

SGTV Speed $-2.5 \mathrm{ft} / \mathrm{s}$

OAS Stopping Distance $-5 \mathrm{ft}$

Target - ITM $45^{\circ}$ angle to SGTV

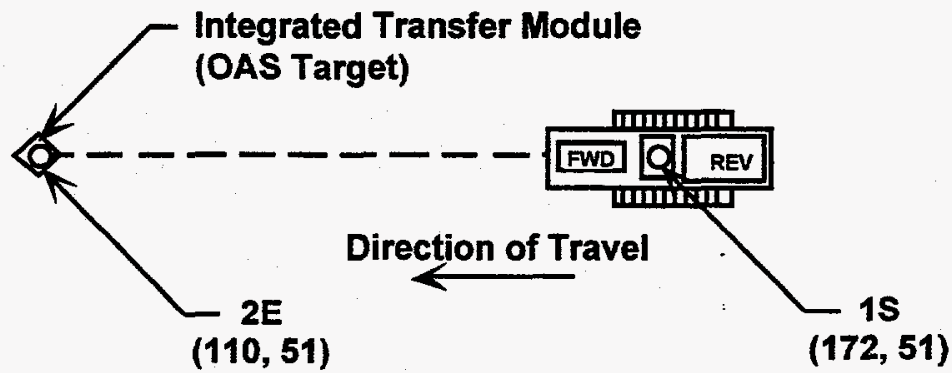

Notes: Trial 4 and 5. OAS recognized the ITM and stopped the vehicle $2 \mathrm{ft}$ after hitting it. 
Table 17. Results of OAS test series number 3.

\begin{tabular}{ccccc}
\hline Trial Number & Detection Zone (ft) & Collision Zone $(\mathrm{ft})$ & \multicolumn{2}{c}{ E-Stop $(\mathrm{ft})$} \\
\hline & OAS & OAS & Tape & OAS \\
\hline 7 & 20 & 19 & 4.3 & 3.6 \\
8 & 21 & 19.8 & 4.1 & 3.3 \\
9 & 23.6 & 19.3 & 3.9 & 3.1 \\
\hline
\end{tabular}

Trial configuration notes:

Mode - Self-guided

Direction of travel - Forward

SGTV Speed - $2.5 \mathrm{ft} / \mathrm{s}$

OAS Stopping Distance - $5 \mathrm{ft}$

Target - Human surrogate stationary

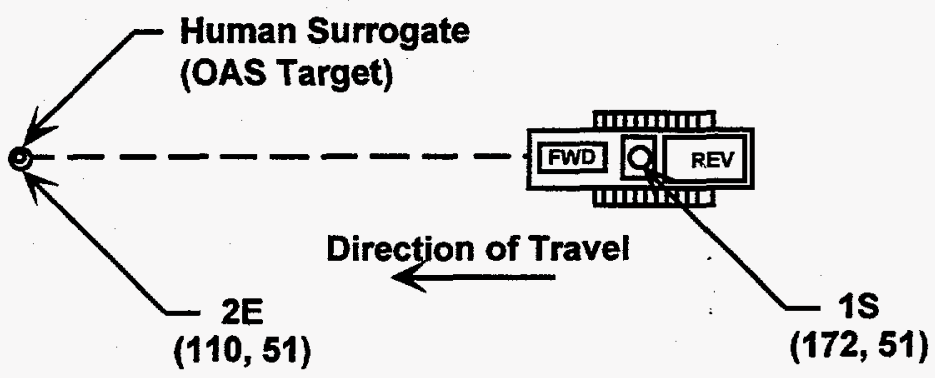

Notes: None 
Table 18. Results of OAS test series number 4.

\begin{tabular}{ccccc}
\hline Trial Number & Detection Zone $(\mathrm{ft})$ & Collision Zone $(\mathrm{ft})$ & \multicolumn{2}{c}{ E-Stop $(\mathrm{ft})$} \\
\hline 10 & OAS & OAS & Tape & OAS \\
\hline 11 & na & na & 4.5 & 3.2 \\
12 & na & 7.4 & 3.0 & 2.4 \\
& na & na & 1.1 & 1.0 \\
\hline
\end{tabular}

Trial configuration notes:

Mode - Self-guided

Direction of travel - Forward

SGTV Speed $-2.5 \mathrm{ft} / \mathrm{s}$

OAS Stopping Distance $-5 \mathrm{ft}$

Target - Human surrogate walking into path of on-coming SGTV at right angle

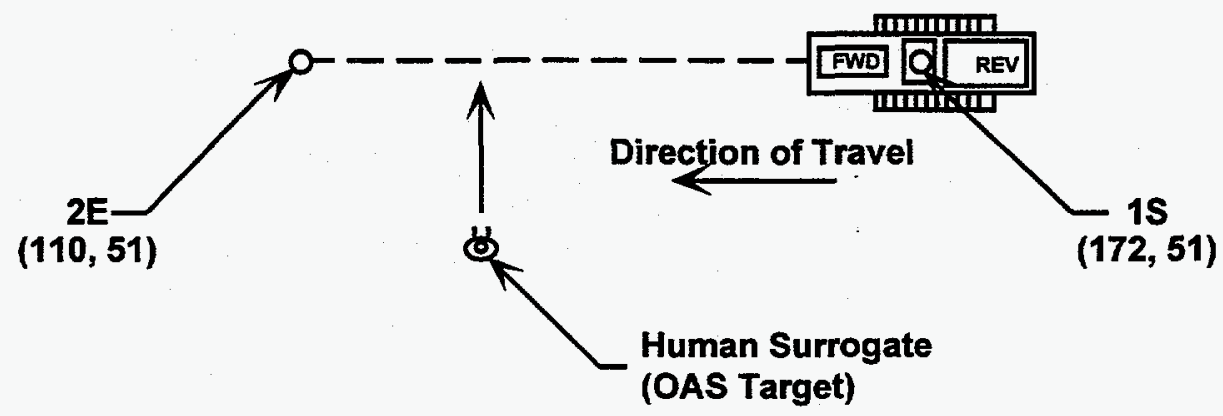

Notes:

Trial 10 - Moved object in when vehicle was $20 \mathrm{ft}$ away

Trial 11 and 12 - Moved object in when vehicle was $15 \mathrm{ft}$ away 
Table 19. Results of OAS test series number 5.

\begin{tabular}{ccccc}
\hline Trial Number & Detection Zone $(\mathrm{ft})$ & Collision Zone $(\mathrm{ft})$ & \multicolumn{2}{c}{ E-Stop $(\mathrm{ft})$} \\
\hline 13 & OAS & OAS & Tape & OAS \\
\hline 14 & 19 & 18 & na & See Notes \\
15 & na & 15.4 & 5.75 & 4.5 \\
& na & na & na & See Notes \\
\hline
\end{tabular}

Trial configuration notes:

Mode - Self-guided

Direction of travel - Forward

SGTV Speed - $2.5 \mathrm{ft} / \mathrm{s}$

OAS Stopping Distance - $5 \mathrm{ft}$

Target - Human surrogate moving away from SGTV in straight line.

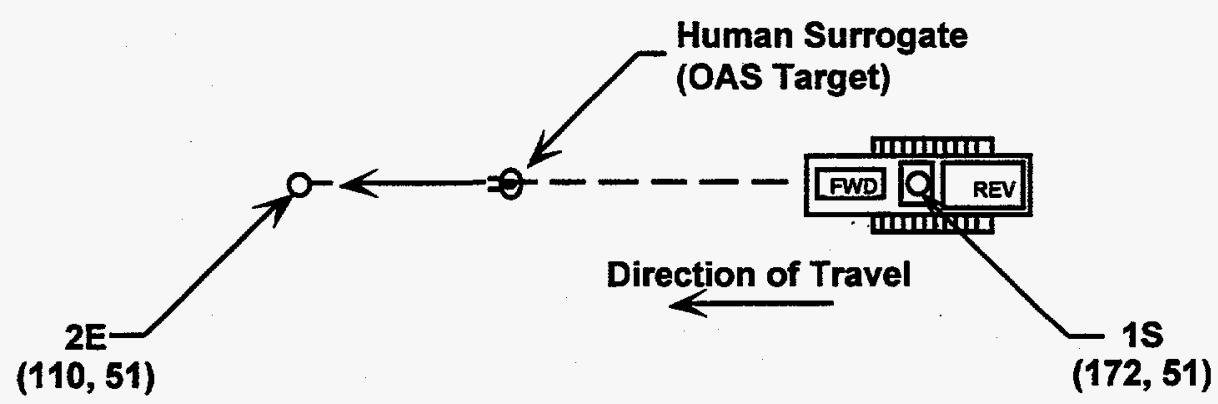

Notes:

Trial 13 - The vehicle stopped approximately $5 \mathrm{ft}$ from the target, but the operator towing the target didn't stop the target. As soon as it was out of the e-stop zone, the vehicle began moving forward again until it re-acquired the target in its e-stop zone and stopped again at approximately $5 \mathrm{ft}$. No exact data was taken because the operator had again moved the target and therefore lost the data point.

Trial 15 - The vehicle stopped approximately $5 \mathrm{ft}$ from the target, but no exact data was taken because the operator had again moved the target and therefore lost the data. 
Table 20. Results of OAS test series number 6.

\begin{tabular}{ccccc}
\hline Trial Number & Detection Zone (ft) & Collision Zone $(\mathrm{ft})$ & \multicolumn{2}{c}{ E-Stop $(\mathrm{ft})$} \\
\hline 16 & OAS & OAS & Tape & OAS \\
\hline 17 & 21.9 & 18.7 & 3.25 & 2.6 \\
18 & 18.0 & 13.0 & 2.0 & 1.4 \\
& na & 15.1 & 1.0 & 1.0 \\
\hline
\end{tabular}

Trial configuration notes:

Mode - Self-guided

Direction of travel - Forward

SGTV Speed $-2.5 \mathrm{ft} / \mathrm{s}$

OAS Stopping Distance - $5 \mathrm{ft}$

Target - Human surrogate moving toward SGTV in straight line.

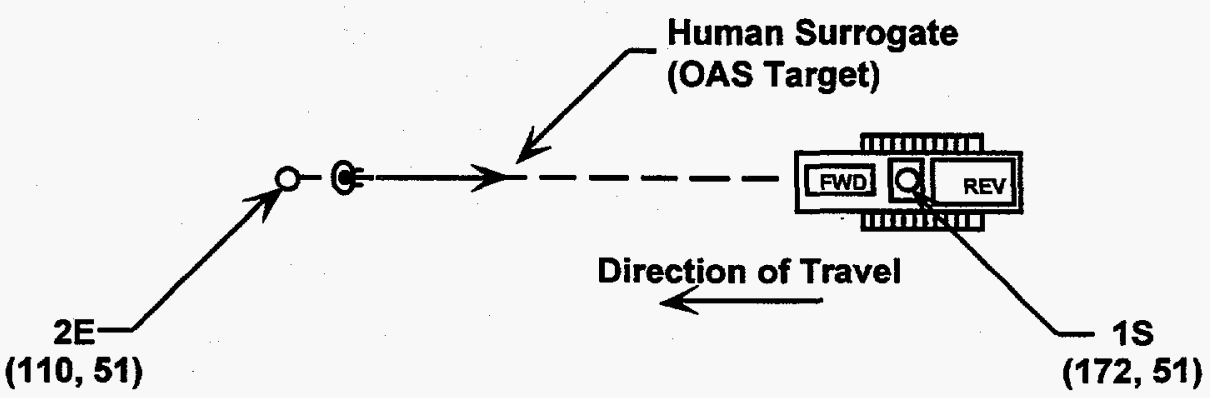

Notes:

Trial 18 - The SGTV stopped before hitting target, but the target rolled off to one side and the SGTV started again after noting that the path was now clear. The target however was right at the corner of the vehicle. The SGTV gently bumped the target before the OAS had time to re-acquire the target and stop the vehicle. 
Table 21. Results of OAS test series number 7.

\begin{tabular}{ccccc}
\hline Trial Number & Detection Zone $(\mathrm{ft})$ & Collision Zone (ft) & \multicolumn{2}{c}{ E-Stop (ft) } \\
\hline 19 & OAS & OAS & Tape & OAS \\
20 & 23.3 & 19.0 & 3.2 & 2.6 \\
21 & 23.5 & 19.3 & 3.5 & 2.8 \\
& 25.0 & 19.2 & 4.0 & 3.3 \\
\hline
\end{tabular}

Trial configuration notes:

Mode - Self-guided

Direction of travel - Reverse

SGTV Speed - $2.5 \mathrm{ft} / \mathrm{s}$

OAS Stopping Distance - $5 \mathrm{ft}$

Target - ITM face on to vehicle.

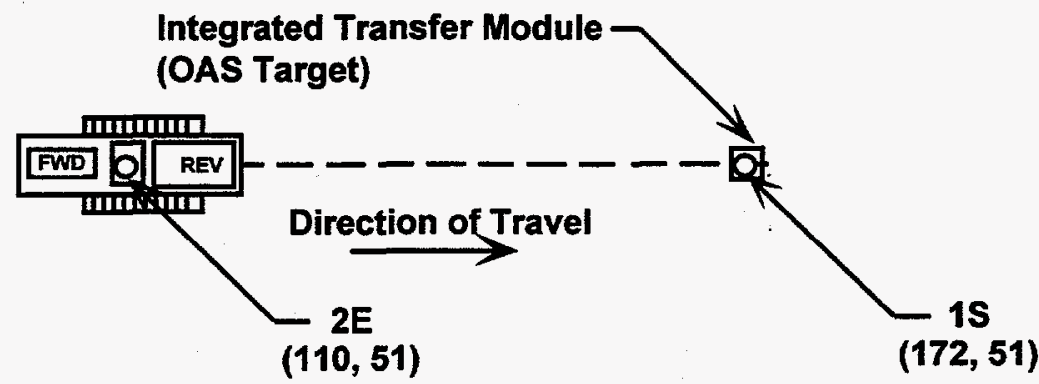

Notes:

None 
Table 22. Results of OAS test series number 8. Note that in this configuration, the OAS system did not detect the object before hitting it.

\begin{tabular}{ccccc}
\hline Trial Number & Detection Zone $(\mathrm{ft})$ & Collision Zone $(\mathrm{ft})$ & \multicolumn{2}{c}{ E-Stop $(\mathrm{ft})$} \\
\hline & OAS & OAS & Tape & OAS \\
\hline 22 & na & na & na & Hit ITM \\
23 & na & na & na & Hit ITM \\
& na & na & na & Hit ITM \\
\hline
\end{tabular}

Trial configuration notes:

Mode - Self-guided

Direction of travel - Reverse

SGTV Speed $-2.5 \mathrm{ft} / \mathrm{s}$

OAS Stopping Distance - $5 \mathrm{ft}$

Target - ITM at $45^{\circ}$ angle to vehicle.

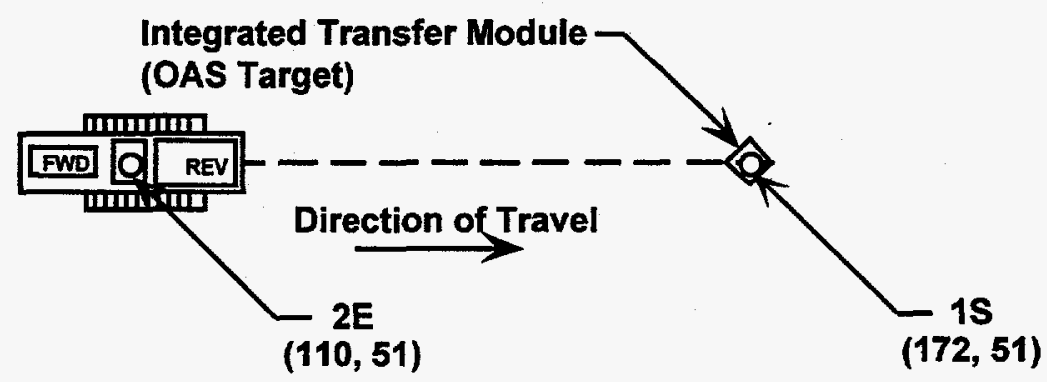

Notes:

Trial 22, 23, and 24 - All three times the vehicle hit the target and moved it approximately $1 \mathrm{ft}$ before stopping. 


\subsubsection{Discussion/Conclusion}

The OAS system was originally intended to operate at $5 \mathrm{~Hz}$. At this rate, it would be capable of quickly detecting and verifying obstacles. Due to a number of factors, the OAS was only capable of operating at $2 \mathrm{~Hz}$. At the reduced speed of the SGTV, this limitation was not much of a factor, but at higher speeds such as existed in the tests where the human subject was moving toward the SGTV, the slower update rates make the OAS performance marginal. With the SGTV moving at full speed ( $5 \mathrm{ft} / \mathrm{s})$ the $2 \mathrm{~Hz}$ update rate combined with the other system latencies may not be adequate to provide safe, reliable obstacle avoidance in all cases.

A significant problem recognizing objects at a certain orientation to the OAS sensors became evident during development. Smooth plane objects oriented at 45 degrees to the SGTV's line of travel proved to be the most difficult to detect. When this problem was discovered, the outer sensors of each bank were angled inward at 30 degrees, and these objects were detected more reliably. However, these objects were usually confirmed later than face-on objects, often resulting in an abrupt stop rather than a smooth slow-down. The test plan was specifically modified to test this senario after it was discovered to be a potential problem. Further analysis and modifications to the sensor array need to be investigated to alleviate this problem.

With the exceptions noted above, the OAS system functioned as designed. It detected objects, cataloged and tracked them, and when necessary halted the vehicle a safe distance from the obstacle. When an object came within the collision zone threshold, the OAS over-rode the velocity commands of the VCCG or the teleoperator and began to slow the vehicle to prepare for a safe stop. When an object no longer presented a potential hazard, the OAS relinquished control and normal operations resumed. One of the biggest concerns about the OAS was whether it would hinder normal operations by interfering with vehicle control too much during maneuvering close to or around objects. Observations made during development and testing showed that the OAS was well integrated with the vehicle control system, does not interfere with operations, and greatly enhances the safety and operational capability of the SGTV.

\subsection{Maneuverability}

Testing performed in FY-94 on the teleoperated version of the SGTV demonstrated the vehicle's basic capabilities to maneuver in rugged terrain, traverse steep slopes, and operate in various soil/surface conditions. The greatly improved hydraulic system and much more powerful engine implemented in FY-95 improved this capability; therefore, no additional testing of this nature was formally planned. However, during control system development it was necessary to perform certain calibration procedures on the electronic compass that required pitching and rolling the vehicle at up to 20-degree angles and performing several tight turning maneuvers. This calibration was performed at RAHCO's facility on hilly terrain in very soft and sandy soil. The vehicle easily drove up and down slopes of 10-20 degrees and performed its turning maneuvers without hesitation (Figure 34). The track grousers, although not designed for operations in the soil conditions present, performed well enough to provide good traction. Optimizing track grousers for specific conditions would improve the SGTV's rough terrain performance. The only limitation on climbing or descending hills is the approach clearance required to account for the fact that the vehicle chassis is significantly longer than the tracks. 


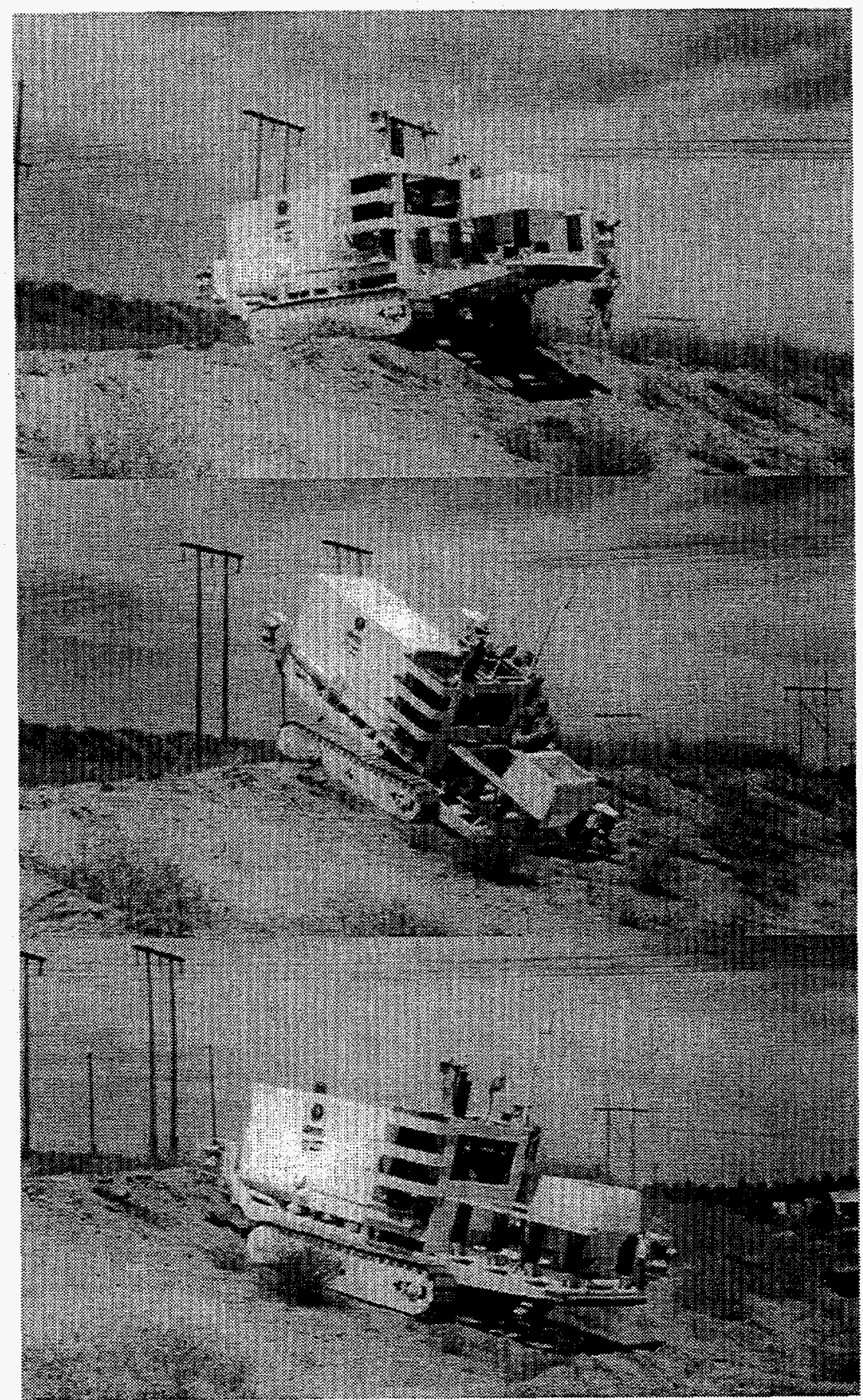

Figure 34. The improved mechanical systems on the SGTV greatly enhanced its capability to maneuver in steep terrain and soft, loose soil conditions. The photos above show the SGTV descending a $20^{\circ}$ slope. (Photo nos. 95-811-1-3, 4, \&5). 
The two maneuverability parameters that were considered to be important in a buried waste retrieval operating scenario are the vehicle's minimum turning radius and its ability to perform a precision docking maneuver. Testing performed included demonstrating the SGTV's turning radius (true and swing area), and its ability to make a close perpendicular approach to a structure, pivot, and dock at a waste transfer station. Measurements were taken using the onboard OAS sensors, a tape measure, and the GPS system. Data from the GPS system is considered to be the most accurate, repeatable, and reliable. OAS data and manual measurements were taken primarily for validation purposes.

\subsubsection{Analysis}

SGTV maneuverability data was gathered from direct measurements. Where appropriate, the mean and standard deviation was calculated. No other analysis of the data was required other than trigonometric calculations of drift and errors from the GPS coordinates.

\subsubsection{Results and Evaluation}

4.7.2.1 Turning Radius. The SGTV, being a tracked vehicle, is capable of turning about its own center. Therefore, the minimum turning radius of the vehicle is defined by its basic geometry. For the SGTV, there are two dimensions of concern for defining the turning radius; the radius of the tracks and the radius of the sweep of the vehicle. The tracks are $11.5 \mathrm{ft}$ long, making the turning radius of the tracks $5.75 \mathrm{ft}$. The overall length of the SGTV is $21 \mathrm{ft}$, making the sweep radius of the vehicle $10.5 \mathrm{ft}$. Since the left and right tracks of the vehicle cannot turn at exactly the same rate, there will be a small amount of drift that will add to the turning radius of the vehicle. A test was performed to examine this drift. The results of that test are shown in the Table 23.

The drift when turning the SGTV clockwise is $1.64 \pm 0.62 \mathrm{ft}$. The drift when turning the vehicle counter-clockwise is $1.76 \pm 1.0 \mathrm{ft}$. Observations during the testing noted that vehicle drift will generally be worse at the slowest turning speed. At very slow turning speeds, the power applied to the tracks is just enough to overcome system friction and move the tracks.

Occasionally, due to the mechanics of the tracks and slight variations in terrain, one track will stick momentarily until the control system reacts to apply increased power to overcome this 'sticktion'. Under these conditions, one track will push the vehicle and pivot it about the other track instead of about the center of the vehicle under normal differential track steering. This phenomena generally doesn't occur at higher turn rates where the power applied to the tracks easily overcomes the system friction.

4.7.2.2 Precision Docking Maneuver. The precision docking maneuver was designed to simulate the situation in which the SGTV would be required to make a precision alignment to a remote operated excavator for loading and unloading (Figure 35). In effect, this is a precision and accuracy test of a docking function. The test was performed in both self-guided and telerobotic mode. The self-guided mode uses the mission path plan from the VCCG and navigates using the GPS/DR system. It does not utilize the targets for navigation and docking. The teleoperated mode uses video cues attached to the wooden target for alignment and distance calculations as well as feedback from the OAS system for distance-to-target information. In an actual dig scenario, these targets would generally not be available. This combined with the simple path makes the teleoperated function relatively simple. Thus, the results of the teleoperated test are better than would be expected in an actual retrieval. 
Table 23. For each trial in the turning radius test, the start and end position was noted from the GPS system. From this, a drift was calculated, that when examined in conjunction with the vehicle geometry, determines the turning radius of the SGTV.

\begin{tabular}{|c|c|c|c|c|c|c|}
\hline Trial & \multicolumn{2}{|c|}{ Clockwise } & $\begin{array}{l}\text { Drift } \\
\text { (ft) }\end{array}$ & \multicolumn{2}{|c|}{ Counter -clockwise } & $\begin{array}{l}\text { Drift } \\
(\mathrm{ft})\end{array}$ \\
\hline $\begin{array}{c}\text { (self-guided) } \\
(3 \mathrm{deg} / \mathrm{s})\end{array}$ & $\begin{array}{l}\text { Start Position } \\
\text { End Position }\end{array}$ & $\begin{array}{l}165.73,49.33 \\
167.53,48.25\end{array}$ & 2.10 & $\begin{array}{l}\text { Start Position } \\
\text { End Position }\end{array}$ & $\begin{array}{l}167.56,48.25 \\
166.91,44.64\end{array}$ & 3.67 \\
\hline $\begin{array}{c}2 \\
\text { (self-guided) } \\
(6 \mathrm{deg} / \mathrm{s})\end{array}$ & $\begin{array}{l}\text { Start Position } \\
\text { End Position }\end{array}$ & $\begin{array}{l}168.09,38.40 \\
168.58,38.11\end{array}$ & 0.57 & $\begin{array}{l}\text { Start Position } \\
\text { End Position }\end{array}$ & $\begin{array}{l}168.68,38.11 \\
169.37,37.75\end{array}$ & 0.78 \\
\hline $\begin{array}{c}3 \\
\text { (self-guided) } \\
(15 \mathrm{deg} / \mathrm{s})\end{array}$ & $\begin{array}{l}\text { Start Position } \\
\text { End Position }\end{array}$ & $\begin{array}{l}166.84,43.59 \\
168.39,42.64\end{array}$ & 1.82 & $\begin{array}{l}\text { Start Position } \\
\text { End Position }\end{array}$ & $\begin{array}{l}168.39,42.64 \\
168.68,42.05\end{array}$ & 0.66 \\
\hline $\begin{array}{c}4 \\
\text { (self-guided) } \\
(\sim 3 \mathrm{deg} / \mathrm{s})\end{array}$ & $\begin{array}{l}\text { Start Position } \\
\text { End Position }\end{array}$ & $\begin{array}{l}162.51,45.23 \\
163.46,45.04\end{array}$ & 0.97 & $\begin{array}{l}\text { Start Position } \\
\text { End Position }\end{array}$ & $\begin{array}{l}163.46,45.04 \\
163.79,42.81\end{array}$ & 2.25 \\
\hline $\begin{array}{c}5 \\
\text { (self-guided) } \\
(\sim 6 \mathrm{deg} / \mathrm{s})\end{array}$ & $\begin{array}{l}\text { Start Position } \\
\text { End Position }\end{array}$ & $\begin{array}{l}163.79,42.81 \\
165.56,42.41\end{array}$ & 1.81 & $\begin{array}{l}\text { Start Position } \\
\text { End Position }\end{array}$ & $\begin{array}{l}165.56,42.41 \\
165.20,41.30\end{array}$ & 1.17 \\
\hline $\begin{array}{c}6 \\
\text { (self-guided }) \\
(\sim 15 \mathrm{deg} / \mathrm{s})\end{array}$ & $\begin{array}{l}\text { Start Position } \\
\text { End Position }\end{array}$ & $\begin{array}{l}165.20,41.30 \\
167.11,40.34\end{array}$ & 2.14 & $\begin{array}{l}\text { Start Position } \\
\text { End Position }\end{array}$ & $\begin{array}{l}167.11,40.34 \\
168.09,38.70\end{array}$ & 1.91 \\
\hline $\begin{array}{c}7 \\
\text { (self-guided) } \\
(\sim 25 \mathrm{deg} / \mathrm{s})\end{array}$ & $\begin{array}{l}\text { Start Position } \\
\text { End Position }\end{array}$ & $\begin{array}{l}170.98,43.46 \\
173.08,43.53\end{array}$ & 2.10 & $\begin{array}{l}\text { Start Position } \\
\text { End Position }\end{array}$ & $\begin{array}{l}173.08,43.53 \\
173.24,41.62\end{array}$ & 1.92 \\
\hline
\end{tabular}




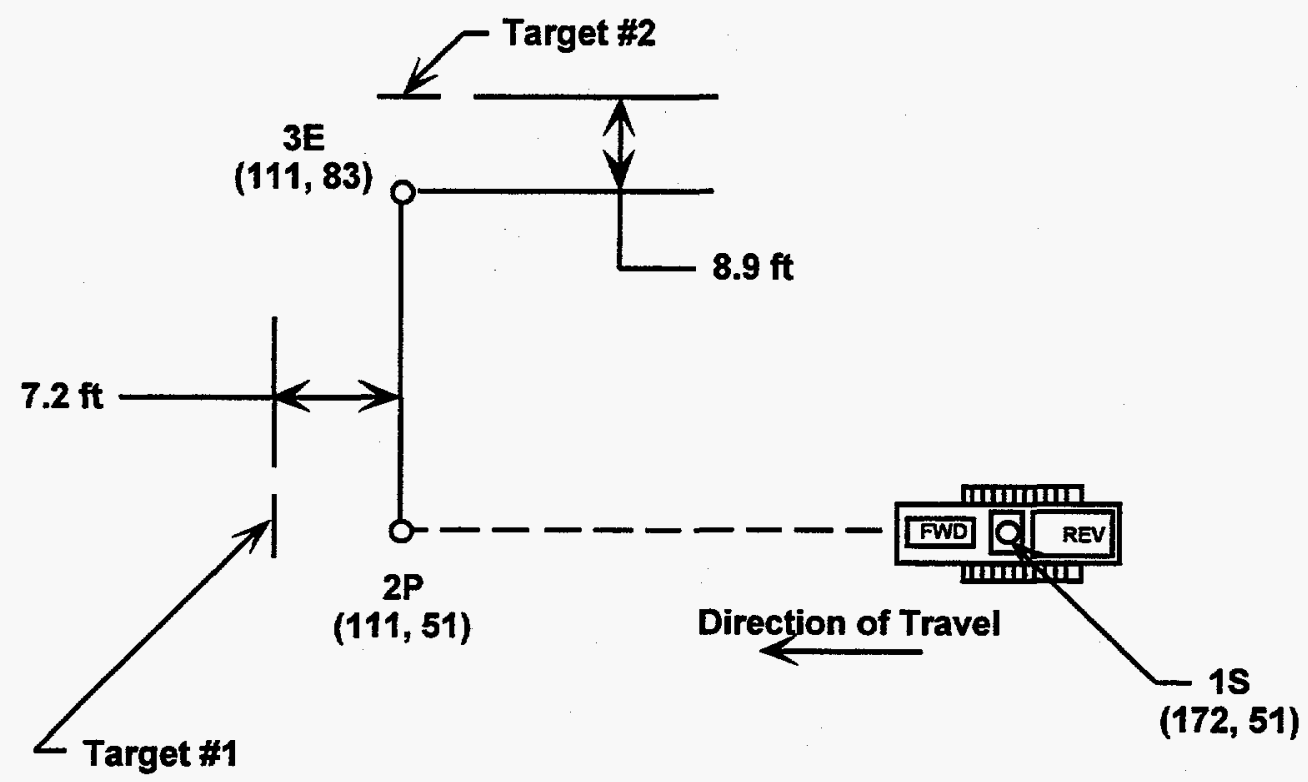

Figure 35. The precision docking maneuver was performed using wooden targets to simulate an excavator or other retrieval equipment and as reference points for verifying the GPS data.

Two test series were run, each with three trials. The first series was performed in selfguided mode. The data for the self-guided test is shown in Table 24. The second series was performed in teleoperated mode. The data for the teleoperated test is shown in Table 25. The important data to look at is the error as determined by the GPS system. The OAS and manual measurements were taken because they were readily available and provide some level of verification. Two data points were measured to determine the accuracy of the docking maneuver. The first is the position relative to point $2 \mathrm{P}$ and the second is the final (docking) position relative to point 3E (refer to Figure 35). The error data presented in the tables is the gross error relative to the target point. In the case of point $2 \mathrm{P}$, the expected error is greater than zero because the pause point diameter is $4 \mathrm{ft}$. The vehicle is commanded to stop when it reaches the pause point circle inducing an error in the direction of travel that, in this case should be between 0 and $2 \mathrm{ft}$. However, the vehicle should be on line in the $y$ direction when it arrives at the target location. (In other words, for point 2P, the expected error in self-guided mode is $x=0.0$ to $2.0 \mathrm{ft}$ and $y=$ $0.0 \mathrm{ft}$ ). Similarly, at point $3 \mathrm{E}$, the expected error in the $\mathrm{y}$ direction should be between 0 and $1 \mathrm{ft}$ because the end point circle is $2 \mathrm{ft}$ in diameter. Therefore, for point $3 \mathrm{E}$, the expected error is $\mathrm{x}=$ $0.0 \mathrm{ft}$ and $\mathrm{y}=0.0$ to $1.0 \mathrm{ft}$. Table 26 presents the data for both the self-guided and teleoperated tests for easier comparison. Note that the expected errors previously discussed only apply to the self-guided tests. The teleoperated mode had no expected error since the operator was instructed to position the vehicle as accurately as possible directly at the target location. The important thing to notice about the data in this table is the accuracy and repeatability of the maneuver in the self-guided mode as compared to teleoperated mode. Even with precise alignment target queues and OAS sensors to help the operator, teleoperated maneuvering is significantly less precise and repeatable than in self-guided mode. 
Table 24. Gross error data for the precision docking maneuver in self-guided mode is shown below. OAS and manual measurements provide only the distance to the target. The GPS provides the $\mathrm{x}, \mathrm{y}$ coordinate of the vehicle in terms of the local coordinate system.

\begin{tabular}{|c|c|c|c|c|c|c|c|}
\hline Trial & Distanc & $\begin{array}{l}\text { to Reference } \\
\text { rget } 1 \\
\text { (ft) }\end{array}$ & $\begin{array}{c}\text { Gross Error } \\
\text { (ft) }\end{array}$ & Distanc & $\begin{array}{l}\text { Reference } \\
\text { get } 2 \\
\text { ft) }\end{array}$ & $\begin{array}{l}\text { Gross Error } \\
\text { (ft) }\end{array}$ & $\begin{array}{c}\text { Angle to } \\
\text { Target } \\
\# 2\end{array}$ \\
\hline 1 & $\begin{array}{c}\text { OAS } \\
\text { Manual } \\
\text { GPS } \\
(x, y)\end{array}$ & $\begin{array}{c}6.0 \\
7.2 \\
111.59,50.68\end{array}$ & $\begin{array}{c}1.2 \\
0 \\
0.59,0.32\end{array}$ & $\begin{array}{l}\text { OAS } \\
\text { Manual } \\
\text { GPS }\end{array}$ & $\begin{array}{c}7.2 \\
8.9 \\
110.41,82.93\end{array}$ & $\begin{array}{c}1.7 \\
0.0 \\
0.59,0.07\end{array}$ & 0.5 \\
\hline 2 & $\begin{array}{c}\text { OAS } \\
\text { Manual } \\
\text { GPS } \\
(x, y)\end{array}$ & $\begin{array}{c}6.2 \\
7.6 \\
111.66,51.34\end{array}$ & $\begin{array}{c}1.0 \\
0.4 \\
0.66,0.34\end{array}$ & $\begin{array}{c}\text { OAS } \\
\text { Manual } \\
\text { GPS }\end{array}$ & $\begin{array}{c}6.6 \\
8.25 \\
109.92,83.49\end{array}$ & $\begin{array}{c}2.3 \\
0.65 \\
1.08,0.49\end{array}$ & 0.9 \\
\hline 3 & $\begin{array}{c}\text { OAS } \\
\text { Manual } \\
\text { GPS } \\
(x, y)\end{array}$ & $\begin{array}{c}6.0 \\
7.3 \\
111.59,51.01\end{array}$ & $\begin{array}{c}1.2 \\
0.1 \\
0.59,0.01\end{array}$ & $\begin{array}{c}\text { OAS } \\
\text { Manual } \\
\text { GPS }\end{array}$ & $\begin{array}{c}7.1 \\
8.75 \\
110.51,83.00\end{array}$ & $\begin{array}{c}1.8 \\
0.15 \\
0.49,0.0\end{array}$ & 0.3 \\
\hline
\end{tabular}

Table 25. Gross error data for the precision docking maneuver in teleoperated mode is shown below. OAS and manual measurements provide only the distance to the target. The GPS provides the $\mathrm{x}, \mathrm{y}$ coordinate of the vehicle in terms of the local coordinate system.

\begin{tabular}{|c|c|c|c|c|c|c|c|}
\hline Trial & Distan & $\begin{array}{l}\text { to Reference } \\
\text { rget } 1 \\
\text { (ft) }\end{array}$ & $\begin{array}{c}\text { Gross Error } \\
\text { (ft) }\end{array}$ & Distanc & $\begin{array}{l}\text { Reference } \\
\text { get } 2 \\
\text { ft) }\end{array}$ & $\begin{array}{c}\text { Gross Error } \\
(\mathrm{ft})\end{array}$ & $\begin{array}{c}\text { Angle to } \\
\text { Target } \\
\# 2 \text { (deg) }\end{array}$ \\
\hline 1 & $\begin{array}{c}\text { OAS } \\
\text { Manual } \\
\text { GPS } \\
(x, y)\end{array}$ & $\begin{array}{c}6.2 \\
7.6 \\
111.79,51.63\end{array}$ & $\begin{array}{c}1.0 \\
0.4 \\
0.79,0.32\end{array}$ & $\begin{array}{c}\text { OAS } \\
\text { Manual } \\
\text { GPS }\end{array}$ & $\begin{array}{c}6.1 \\
7.5 \\
110.25,84.18\end{array}$ & $\begin{array}{c}2.8 \\
1.4 \\
0.75,1.18\end{array}$ & 0.2 \\
\hline 2 & $\begin{array}{c}\text { OAS } \\
\text { Manual } \\
\text { GPS } \\
(x, y)\end{array}$ & $\begin{array}{c}4.6 \\
5.9 \\
109.85,51.07\end{array}$ & $\begin{array}{c}2.6 \\
1.3 \\
1.15,0.34\end{array}$ & $\begin{array}{c}\text { OAS } \\
\text { Manual } \\
\text { GPS }\end{array}$ & $\begin{array}{c}3.7 \\
4.7 \\
109.76,86.93\end{array}$ & $\begin{array}{c}5.2 \\
4.2 \\
1.24,3.93\end{array}$ & 0.7 \\
\hline 3 & $\begin{array}{c}\text { OAS } \\
\text { Manual } \\
\text { GPS } \\
(x, y)\end{array}$ & $\begin{array}{c}4.5 \\
5.4 \\
109.69,51.20\end{array}$ & $\begin{array}{c}2.7 \\
1.8 \\
1.31, .20\end{array}$ & $\begin{array}{c}\text { OAS } \\
\text { Manual } \\
\text { GPS }\end{array}$ & $\begin{array}{c}5.2 \\
6.4 \\
109.59,85.19\end{array}$ & $\begin{array}{c}3.7 \\
2.5 \\
1.41,2.19\end{array}$ & 1.7 \\
\hline
\end{tabular}


Table 26. The results of the positioning errors for the precision docking maneuver are shown below.

\begin{tabular}{cccc}
\hline Trial & $\begin{array}{c}\text { Target 1 } \\
\text { Gross Error }(\mathrm{ft})(\mathrm{x}, \mathrm{y})\end{array}$ & $\begin{array}{c}\text { Target 2 } \\
\text { Gross Error }(\mathrm{ft})(\mathrm{x}, \mathrm{y})\end{array}$ & $\begin{array}{c}\text { Angle to Target 2 (deg) } \\
\text { (final angle to docking } \\
\text { station) }\end{array}$ \\
\hline SG 1 & $0.59,0.32$ & $0.59,0.07$ & 0.5 \\
SG 2 & $0.66,0.34$ & $1.08,0.49$ & 0.9 \\
SG 3 & $0.59,0.01$ & $0.49,0.0$ & 0.3 \\
TO 1 & $0.79,0.32$ & $0.75,1.18$ & 0.2 \\
TO 2 & $1.15,0.34$ & $1.24,3.93$ & 0.7 \\
TO 3 & $1.31,0.20$ & $1.41,2.19$ & 1.7 \\
\hline
\end{tabular}

An important note: Each of the three self-guided trials experienced a network error (crash) at approximately $10 \mathrm{ft}$ beyond point $2 \mathrm{P}$. The network reset, but this did have an adverse effect on tracking accuracy particularly as applies to the final position of the vehicle at point $3 \mathrm{E}$. A slight off-track error was introduced that due to the short length of the path did not allow time for the vehicle to fully recover and eliminate the error. The cause of the network error was not determined, but because of the locational repeatability it is believed that it is related to electromagnetic interference. The network error is discussed in more detail in Section 5, "Discussion, Conclusions, and Recommendations." 


\section{DISCUSSION, CONCLUSIONS, AND RECOMMENDATIONS}

\subsection{Mechanical Systems}

\subsubsection{Effectiveness}

5.1.1.1 Vehicle Structure/Cradle. The SGTV's $10 \times 2$-in. structural tube frame proved to be very stout and withstood all conditions of performance testing.

The SGTV track assemblies with their flat grosser pads operated as expected on asphalt and soil surface conditions. In soil, the flat-steel grosser pads allowed the SGTV to do pure rotational turns with minimal soil plowing, yet supplied enough traction for forward and reverse travel. On asphalt the flat steel grosser pads reduced ground pressure and allowed the SGTV to maneuver with minimum surface wear to the asphalt. The steel growers and rigid frame caused considerable vibration, which at speeds over $2.5 \mathrm{ft} / \mathrm{sec}$ increased dramatically.

Due to the constant vehicle parameter tuning, the SGTV was doing an extreme amount of pure rotational turns at various speeds. This caused the track chains to stretch and loosen to the point that the SGTV had reduced maneuverability. The chain take-ups were adjusted and the SGTV maneuverability was regained.

The SGTV's ITM cradle assembly was very effective in allowing the ITM to self-align to the REMEX end effector. The cradle locks arms provided stability to the cradle and eliminated the bouncing of empty ITMs when the vehicle is in motion.

5.1.1.2 Power Plant/Hydraulics. The 100-hp diesel engine supplied more than enough power to the SGTV track drives for maneuvering in all the required terrain. Throughout the testing the engine was very dependable, with the exception that the radiator developed a small leak. This leak was located at the radiators bottom mounting bracket and was caused by engine oscillation from the SGTV's movement over the terrain. The engine isolator mounts combined with the radiators isolators amplified this oscillation causing the bracket's welded joint to fatigue fail. The radiator was replaced and hard mounted to the frame.

The diesel power plant is directly coupled to a triple pump drive gearbox, which powered two hydrostatic drives and one variable displacement pump. This system proved to be very effective in hydraulically powering all the mechanical systems of the SGTV. The two servo-controlled hydrostatic drives provided excellent mechanical control for the vehicle. The variable displacement pump supplied power to the auxiliary hydraulic functions. All of the auxiliary functions worked well and were very dependable. The hydraulic packaging, hose runs, and tube runs are very clean and functional.

\subsubsection{Human Factors}

The human factors from a mechanical maintenance point of view include the ease of maintenance and frequency of maintenance.

Maintenance required on the SGTV included adjustment of the track assemblies, lubricating the track bearings, changing the engine oil, and installing a new radiator. The track 
chains were adjusted using the track take-up, which is easily accessible and not difficult to adjust. The track bearings and gearbox grease fittings are easily accessible and simple to lubricate. The diesel and hydraulic packaging is very compact and most everything is easily accessible for performing maintenance on the engine. There is a solid guard plate attached to the side of the engine skid frame that makes access to the hydraulic pumps difficult.

\subsubsection{Recommended Improvements and Future Work}

Without further analysis it is impossible to determine the exact cause of these other crashes, but it appears they are related to electro-magnetic interference being generated by other nearby equipment. A great deal of time and effort were expended during the development phase by some highly qualified electrical engineers to analyze and solve the problem.

The SGTV is built on a very stiff structural frame. The steel tracks are mounted directly to the frame. There is no suspension system to dampen shock and vibration. This high vibration environment is the most significant threat to the long term reliability of the SGTV. Structurally the SGTV is extremely durable, however any production version would require some redesign to reduce the vibration from the tracks.

To minimize vehicle noise and vibration, the SGTV track assemblies should be replaced with a rubberized track system.

To prevent further radiator damage the diesel engine should be directly mounted to the skid frame. This would eliminate all oscillations created by the SGTV's motion.

The guard plate mentioned above should be of the removable type to provide access to the hydraulic pumps.

\subsubsection{Conclusions}

The SGTV mechanical/structural system and the functional layout of the vehicle were designed to provide a highly specific function based on a set of requirements that may or may not have a foundation in reality. The truth of the matter is that although the SGTV is a well designed and built vehicle, the mechanical systems and design of the vehicle are of minor importance to the overall technology. The SGTV technology can be applied to many types and styles of vehicles. This particular vehicle was designed for transuranic buried waste retrieval using remote operated excavation equipment. An entirely different application would most certainly result in a specification that would lead to a significantly different mechanical design. It should also be noted that this vehicle was designed to prove the basic approach and technologies for self-guided transportation of buried transuranic wastes. A vehicle that was specifically intended for production operations in a transuranic retrieval operation would require some specific upgrades to increase its reliability, make it easier to decontaminate and possibly convert it to all electric operations. These are considerations that were above and beyond the scope of the technology demonstration. 


\subsection{Control System and Electronics}

\subsubsection{Effectiveness}

5.2.1.1 Adequacy and Reliability of Controls. SGTV machine control can be assessed both in terms of critical risk to personnel and equipment in the operating area, long-term ease of use, and operation and overall ruggedness and reliability.

The SGTV was in safe control at all times during development and final testing. Risk management encompasses several factors:

- Operator/Supervisor hydraulic cutoff should the vehicle appear to be at risk

- Supervisor remote emergency stop of the SGTV using the REMTRON radio control system

- Direct emergency stop using the accessible ESTOP control mounted on the vehicle itself

- Automatic emergency stop should telemetry or network communications be lost for a predetermined period of time

- Automatic emergency stop if off-track deviation reaches unacceptable levels

- Filtering of unusually large feedback deviations from on-board sensors

- Limiting of operator velocity input and velocity and acceleration output to control valves

- Automatic deceleration and stopping of the vehicle when OAS sensor report objects ahead of the SGTV.

5.2.1.2 Adequacy and Reliability of Sensors. Sensor reliability is closely coupled to control reliability and proves to be the most sensitive to tuning. This is particularly true in the case of the redundant on-board sensors (compass, gyro and track encoders) which provide for fault tolerant operation but also significantly increase the complexity of the tuning process.

Perhaps the most valuable feature of the Tempo operating system used for system control is the near total accessibility of its parameter tables. This greatly facilitates tuning of various sensor weights.

The control system nevertheless remains vulnerable to infrequent periods of satellite loss in the GPS positioning system. At the INEEL facility, these were typically of short duration, occurring no more than twice a day for periods of 30 to 60 minutes. Since the differential GPS system is the only external positioning sensor, its degradation requires temporary suspension of operations.

Ideally, only the compass and the track encoders are necessary to provide a dead reckoned position between GPS fixes. In practice however, the present compass is too sensitive to 
environmental distortion to be relied upon as an single source of heading and angular velocity. It has been found that a weighted combination of all on-board sensors and, optionally, a heading derived from change in GPS position, are required for adequate heading estimation.

5.2.1.3 Obstacle Avoidance and Personnel Risk. The Obstacle Avoidance System (OAS) is implemented on the SGTV onboard controller. The OAS can detect stationary or moving objects in the direction of motion of the vehicle. When an object is detected, it can determine, based on the obstacle range, whether just to inform the operator, slow down, or stop the vehicle. The VCCG displays the OAS status to the operator.

The OAS system performed within expected limits, providing a valuable means of sensing unpredictable events in the working area. As reported by other researchers, smooth plane objects oriented at 45 degrees to the SGTV's line of travel proved to be the most difficult to detect. When the outer sensors of each bank were angled inward at 30 degrees these objects were detected more reliably, but were usually confirmed later than face-on objects, often resulting in an abrupt stop rather than a smooth slowdown.

The nine sensors pointed forward and nine in reverse provided adequate coverage of the SGTV's path and reliably detected most objects down to the size of small-diameter rod.

A dummy constructed to imitate a typical person was effectively detected under all test situations. The area of greatest risk remains the sudden appearance of objects moving from along side the vehicle directly into the line of travel and objects encountered during high speed rotation of the SGTV.

The OAS system was originally intended to operate at $5 \mathrm{~Hz}$. At this rate, it would be capable of quickly detecting and verifying potential obstacles. However, in its final incarnation, the OAS was only capable of operating at $2 \mathrm{~Hz}$, which is marginal for providing reliable, safe operations.

5.2.1.4 Environmental Sensitivity. Final testing of the SGTV was done at speeds of about 2.5 fps. A variety of complications in the testing area, ranging from active arc welding equipment to multiple sources of radio interference, provided the opportunity to test SGTV control systems in a highly complex environment. There was some apparent sensitivity of the onboard electronics to unresolved electromagnetic interference under certain conditions and at particular times of day. Spread spectrum radio communications were for the most part tolerant of such interference but appeared to be somewhat sensitive to antenna placement. Emergency stop radio operation was reliable throughout the test area regardless of operating conditions.

\subsubsection{Human Factors}

Most of the on-board electronics are housed in a single box. Access to the box is easy, but annoying due to the number of latches. In general, accessibility to the on-board control system was not considered as important as sealing systems from the environment. Modifications or repairs to the on-board electronics usually requires disconnecting the box from the SGTV and bringing it to a workbench. This process can be accomplished easily and in less than 10 minutes.

The on-board mode switch (to change the vehicle from off to local or remote operations) was difficult to reach. The clearance between the electronics box and the GPS box made 
connecting and disconnecting several of the wiring harnesses (especially the pendant controller) difficult.

Video reception was adequate under most conditions, the low-light capability of the onboard cameras being so good that the vehicle appeared to be operating in day-light conditions after sundown with only yard lighting. Video reception was not good in all parts of the test area and interference prevented optimum viewing of vehicle operation from the on-board camera at times.

Operation of the remote-control REMTRON emergency stop system was almost immediate, and, despite being sensitive to excessive tilt of the hand-held radio unit, worked well at all times.

\subsubsection{Recommended Improvements and Future Work}

The on-board control system proved defective in two areas:

- Driving the SGTV at top speed resulted in dropouts of on-board network communications; the cause seemed to be due to an electronic fault aggravated by the severe vibrations caused by the steel tracks on the pavement.

- Indication of engine speed became unreliable toward the end of testing. The sensor seems to be quite exposed and of inadequate frequency response.

It is clear that the ability of the SGTV's on-board electronics to withstand severe continual vibration could be improved and the question of vulnerability to a variety of electromagnetic interference addressed with an eye to better shielding of enclosures and exposed cabling.

The performance of the differential GPS external positioning system was, on the whole, encouraging, but the latency and frequency of the Trimble system remains a serious limitation to better performance of the navigation system. Newer GPS units capable of much higher frequency and lower latency are now available and would undoubtedly improve SGTV operation.

If the present compass were to be replaced by compass based on a different principle of operation, or if it could be better protected from environmental magnetic distortion, the overall reliability of the estimated heading would improve and the effect on SGTV performance may be noticeable.

The obstacle avoidance system can be made more effective by providing more cross-path sensors to detect oblique smooth surfaces and by adding side sensors that scan the sides of the vehicle to pick up objects appearing to move rapidly across the line of travel either due to their own speed or due to rotation of the SGTV itself. The update rate of the OAS must be increased beyond the $2 \mathrm{~Hz}$ rate of the current system to support higher-speed vehicle operations and to improve reliability and responsiveness of the system.

Access to the debug output from each of the five on-board computers and therefore the ability to edit parameters during tuning currently requires a serial wire link. A radio link to the debug port would greatly shorten future tuning periods and allow 'on-the-fly' adjustment and testing of critical parameters. 


\subsubsection{Conclusions}

The accuracy of the SGTV control system is presently high enough to meet specifications for path following and docking repeatability, but the reliability of the electronic controls can be improved by addressing issues of vibration tolerance and better electromagnetic shielding. Replacement of present generation sensors with newer and more ruggedized versions would prove useful in further enhancing accuracy of the positioning and navigation system.

\subsection{Mission Planning and Vehicle Command and Control Generator}

\subsubsection{Effectiveness}

5.3.1.1 Computer System. The effectiveness of computer system, consisting of hardware and software, is discussed below.

Hardware-The original proposal was for a $486 \mathrm{DX}$ computer with $8 \mathrm{MB}$ RAM and a 19 in. monitor. After a careful review of the requirements, it was decided to substitute a Pentium $75 \mathrm{MHz}$ computer (Intel motherboard and CPU) with $16 \mathrm{MB}$ RAM and a 17-in. monitor. The cost is approximately the same because the saving on the monitor covers the upgrade of the CPU and RAM. The change enhanced the VCCG capability significantly, particularly the GUI. The 17-in. Sony color monitor has a very clear and sharp image. It accommodates all the required icons and the map of the worksite, and the operator can access the icons easily. The Western Digital hard drive was one of the best IDE drives available at the time, and it has $256 \mathrm{~Kb}$ cache memory which significantly speeds up the data logging in real time control. A keyboard and mouse are the input devices for MP and VCCG. Since they are the basic input devices for all personal computers, no extra training was required.

The VCCG computer is an off-the self industry standard computer that is powerful, yet low-cost with spare parts easily obtained. During system development at Victoria, BC, Spokane, WA and Idaho Falls, ID, no problems were encountered.

Software-The software used to develop the MP and VCCG was Microsoft Visual Basic. There are many off-the-self GUI modules available; as a result, the development cost was reduced without compromising the quality of the graphics. The major benefit of the Windows-based software is that many people are familiar with windows operations, therefore a novice operator should be able to run the MP or VCCG with less than $1 / 2$ hour of training.

The communication software for interfacing with the telemetry router was coded in Visual $\mathrm{C}++$ because it was found to be more efficient than the Visual Basic version.

During the VCCG operations, data logging requires frequent hard disk access. To ensure the data is not corrupted and that hard disk capability is optimized, the hard disk was checked and optimized frequently using SCANDISK and DEFRAG routines.

5.3.1.2 Mission Planning. The MP software is a very user-friendly package. New operators were able to become familiar with all the functions with less than $1 / 2$ hour of training. 
The worksite map can be imported easily in Windows bitmap format. Two worksite maps, one for RAHCO's test site and one for the INEEL, were implemented. In both maps, all the obstacles and borders were clearly delineated. The GPS was used to calibrate the map and achieved accuracy of better than \pm 2 in. The layout of the icons and buttons was logical and thus easy to use.

During the testing, the operator created the no-go zones within 13 minutes, among which most of the time was spent on discussion. The no-go zones, after the file has been stored, can be edited to add or delete no-go zones easily. Buffers between the free space and the no-go zones can also easily be added and modified.

Three paths were created for testing, ranging from simple to complex (Figures 36, 37, and 38). It took 7, 18, and 15 minutes to create the simple, medium, and complex paths, respectively. Most of the time was spent on selecting suitable locations for the waypoints. For this reason, it took more time to create a medium difficulty path than the complex path. After completing a path, the collision detection function was used to check the path. As a result of the collision detection, the waypoints were moved when necessary to eliminate possible collisions with known objects. The collision detection function of the MP software did not include a full model of the VCCG kinematic simulation. Consequently, at some turning points, the collision check was too conservative.

\subsubsection{Graphical User Interface for Self-guided Control. The GUI is very}

user-friendly; normally, it takes less than $1 / 2$ hour to be familiar with all the functions. The colors selected for command buttons and windows were selected to provide information to the operator and enhance the aesthetics of the display.

The central part of the command window displays the site map on a 10-ft grid, no-go zones, planned path, actual path, and a representation of the SGTV. The command buttons are all located on the right-hand side of the window. The displays are located on the left-hand side and the analog-gauges and a message box are at the bottom of the screen. The health status of telemetry, network, compass, and the GPS are displayed permanently below the map. The overall health status is shown in a separate window and the status of each element can be viewed by clicking a button. This approach avoids overloading the operator with too much information during normal operation.

The displayed engine speed was not consistent with the actual speed. It was believed that the sensor was not adequate and not a problem with the GUI.

The user parameter editor was very convenient for tuning and selecting control parameters to fit a specific task. It is a regular text editor and the operator is cautioned before opening the parameter file about potential consequences of making changes. This version of the GUI does not require a password to access to the parameter editor. It is recommended that a password to be added in the next version.

The data logging feature is extremely useful for development and testing. All the required data, including time, can be stored and converted to ASCII format for off-line analysis. The data can be plotted using the plotting program included in the GUI. The whole screen can also be captured using a screen capture routine. These capabilities reduced the test time and the workload of the test engineers significantly. 


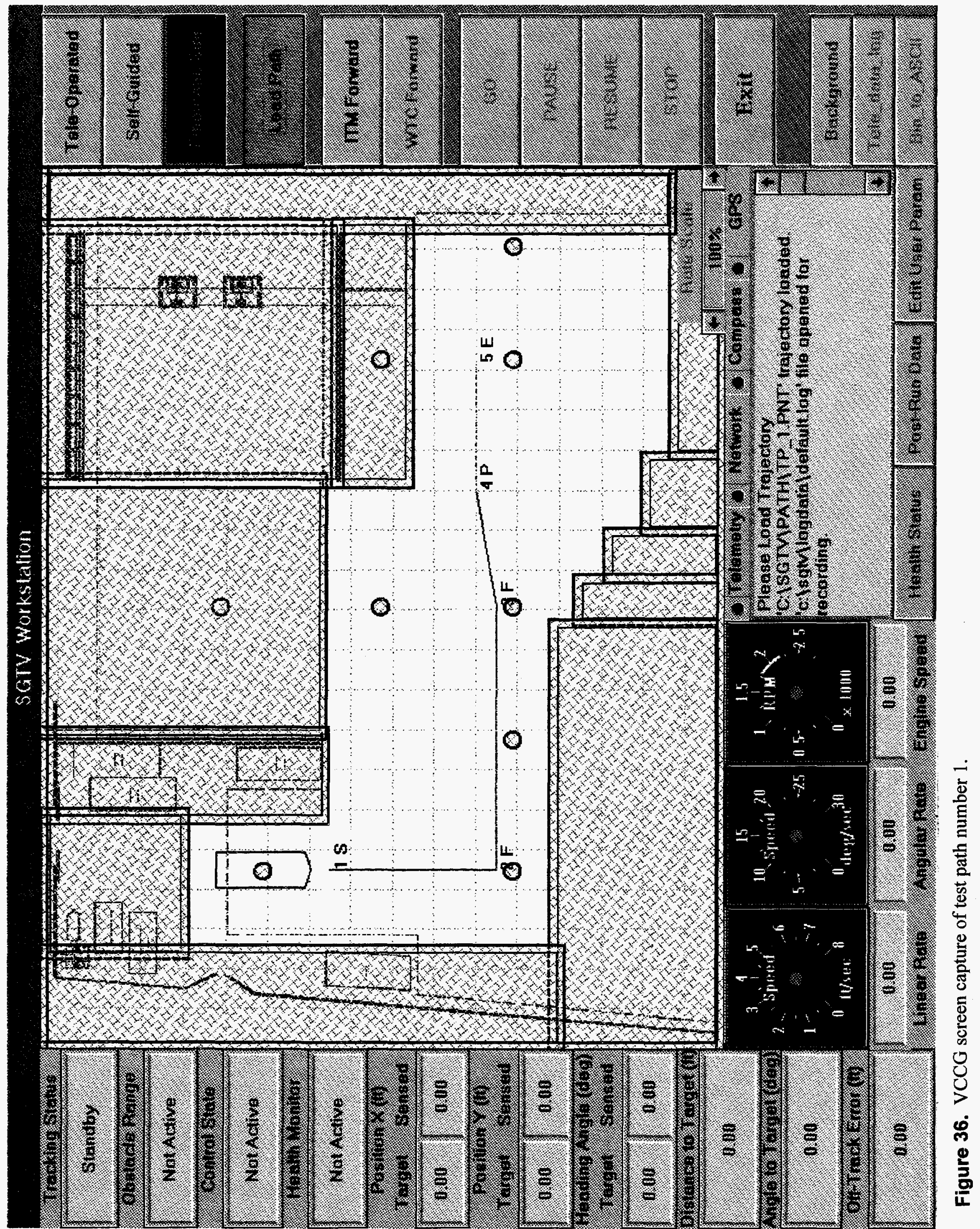




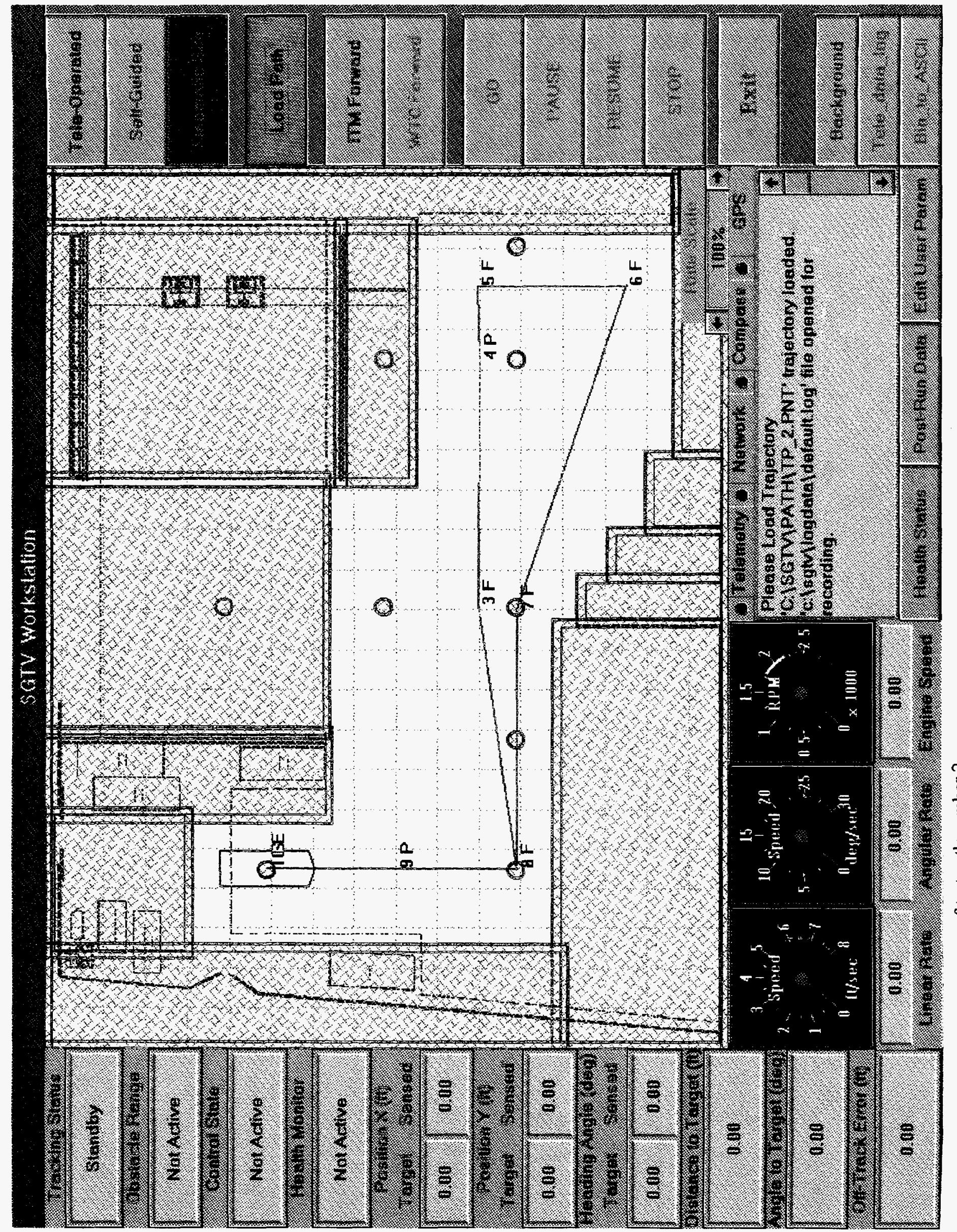

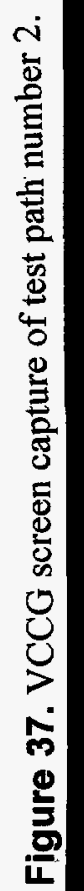




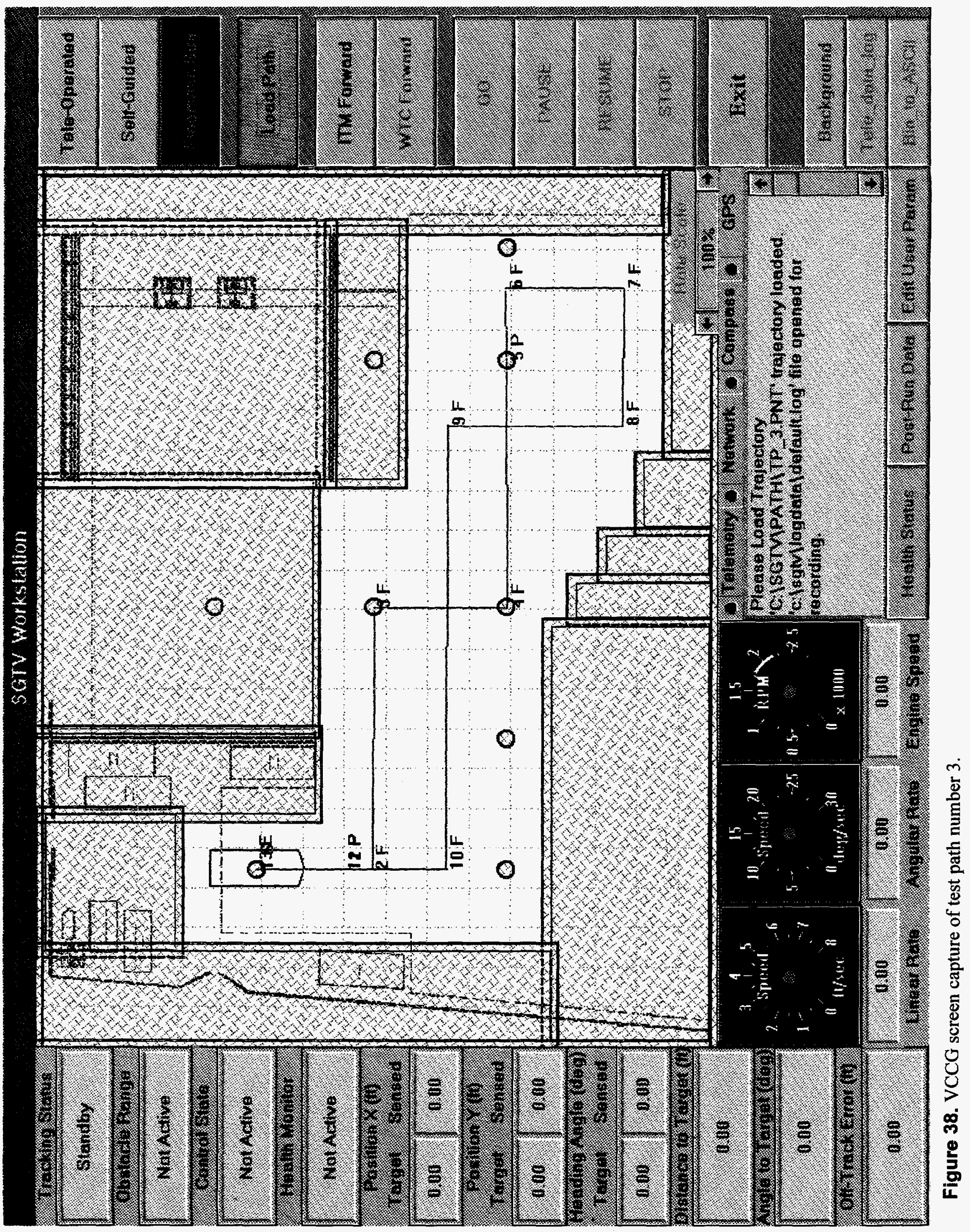


The GUI also assisted with the calibration of the electronic compass. The data required for calibration were the compass readings, and the positions of the vehicle at both ends of a straight path. Normally, this requires measuring the SGTV position in the field. By using the GUI, the vehicle was operated in the teleoperated mode and the data were taken from the screen directly. The process was very smooth, efficient, and much safer than having extra personnel working around the vehicle.

In its first incarnation, the no-go zones covered the underlying map; as a result, the operator could not see the objects behind the no-go zones. The background of the no-go zones was later changed to be transparent and overlaid with a cross-hatched pattern. This layout enables the objects behind the no-go zones to be seen. This change was one of many incorporated as a result of user feedback on the design of the GUI during the development process.

The health monitoring functions enabled the operators to monitor the health status of the vehicle. The red and green alert lights were more effective in alerting the operator to system health status than the text message displayed in the message box.

The "beep" to alert the operator of critical failures or before initiating vehicle movement was very effective. For example, after the operator would click the "GO" command, he would be informed to first obtain permission from the Operation Supervisor to proceed with the operation. In such a event, both a "Beep" and a message are issued.

A new feature added to the GUI was to convert the logged data to ASCII format for export to a spreadsheet program for off-line analysis. This feature was extremely useful for analysis of the test data.

\subsubsection{Self-guided Control.}

This section addresses the self-guided control with respect to the following areas:

- Command and Display

- Interchange between self-guided mode and the other modes

- $\quad$ Effectiveness of waypoint features

- Transient state performance

- Steady-state performance

- Collision avoidance

- Failure protection.

The VCCG algorithms were initially tested on a MATLAB simulation model, then verified using kinematic simulations on the full VCCG software platform. Finally, the VCCG was integrated with the other SGTV subsystems and tested with the vehicle. The quantitative performance analysis is discussed in Section 4 . This section concentrates mainly on system performance issues of the VCCG. 
Command and display - The operator normally uses the mouse to push or release the command buttons. As a backup, the keyboard can also be used. The command buttons are organized to match the operation sequence. No problems were noted in issuing commands through the mouse, and in general the operators found the interface to be well organized. The operator can also change the magnitude of the velocity command through the mouse. This feature allowed the operator to improve the tracking performance. For example, in some test site areas EMI appeared to be a significant factor in vehicle performance. Under these or similar conditions, the operator can reduce the linear speed, as a result, the off-track error may be reduced.

Among all the data and messages displayed on the screen, the operator concentrated mainly on the actual vehicle path versus the planned path shown on the map. The health status of the telemetry, network, compass and GPS are displayed at the center near the bottom of the screen. The operator is alerted to a problem when an LED is changed to red, which indicates an error. The obstacle range was also a very important parameter for the operator to monitor during operations. For example, when the vehicle slows down suddenly, it may be a result of the collision avoidance system commanding a reduced velocity, not a failure of the vehicle or the control system.

Interchange between self-guided mode and the other modes-The VCCG has a feature which allows the operator to switch to another control mode such as Direct Track, Tele-Robotic or Lid Latch/Open, then come back and resume the tracking starting from the current position of the SGTV. It does not need to re-load the planned path or re-initialize the vehicle. This feature was used frequently during the test runs.

Effectiveness of waypoint features-The design of the waypoint features was inspired by the Space Station Remote Manipulator trajectory control. A waypoint, except for the first and the last point which must be the start point and the end-point respectively, can be defined as "pause", "flyby" or "smooth flyby" points. These points are extremely useful in achieving high-performance tracking. It was found that the docking accuracy and stability can be improved significantly if a "pause" point was set just before the end point. This allowed the SGTV to make precise course corrections just before docking. A "pause" point can also be the location for loading and unloading waste along a path. The "Flyby" point is used to achieve tight control for switching from one straight path to the other, but needs more operation time and free space. "Smooth Flyby" points, on the other hand, reduce operation time and can avoid any head-on obstacles. Using smooth flybys was very effective for transitioning between straight path segments, especially when off-track error is not critical.

The "Washin" (accelerating) and the "Washout" (decelerating) circles of the waypoints are very effective in reducing the overshoot of the tracking. The radii of the "Washin" and "Washout" circles can be defined by the operator. For this test, they were set to $5 \mathrm{ft}$ and $10 \mathrm{ft}$, respectively.

Transient state performance-The transient state performance was first tested by commanding step linear rate commands and angular rate commands separately. The vehicle rates are derived from the two track sensors and the rate gyro sensor. The minimum linear acceleration and deceleration are about $1.8 \mathrm{ft} / \mathrm{s}^{2}$ and $3.6 \mathrm{ft} / \mathrm{s}^{2}$ respectively, whereas the minimum angular acceleration and deceleration are about $12 \mathrm{deg} / \mathrm{s}^{2}$ and $24 \mathrm{deg} / \mathrm{s}^{2}$ respectively. With high rate commands, e.g. $5 \mathrm{ft} / \mathrm{s}$ and $15 \mathrm{deg} / \mathrm{s}$, actual vehicle speed is greater than the commands in steady 
state. The discrepancy is more significant in the angular rate response. It appears that the integrator of the track rate servo cannot eliminate the steady state error fast enough. The problem may be solved in the VCCG by scaling the command inversely proportional to its magnitude. This is a low-risk technique because it will not cause stability problems. Normally, integral controllers can eliminate steady state error but may have stability problems due the noticeable time delay at the VCCG command level.

The performance of path tracking during startup was mainly affected by the initial heading error and the linear rate of the vehicle. Therefore it was essential to reduce the initial heading error, using pure rotation, before moving forward. During testing, pure rotations could achieve an initial heading accuracy of \pm 5 degrees or better most of the time. There were a few instances when one of the tracks was stalled then started to move suddenly, which induced a significant heading error. The cause of this phenomenon was not identified, but appears to be related to mechanical resistance and the response of the servo controller to overcome that resistance. The same situation occurred both in the telerobotic mode and the self-guided mode. As a result, the vehicle turned to one side suddenly for a half second (approximately a $10^{\circ}$ overshoot) and then corrected its heading back to normal.

Steady-state performance-Based on the results of the 30 test runs ( 10 for each of the simple, medium, and complex paths), the vehicle was (on average) able to track the planned paths within the $\pm 1 \mathrm{ft}$ off-track error goal in steady-state under normal conditions. The final docking always achieved a position error of less than $1 \mathrm{ft}$ and the heading was locked on target orientation. However, when the vehicle experienced EMI from the other systems being integrated and tested at the same site, the off-track error occasionally exceeded $1 \mathrm{ft}$. The EMI varied with time and location. During the testing of path 1, EMI was very strong around the first point (used both as the start point and end point). As a result, the off-track error was significant and consistent at this point. At the same location and using the same path but executed two days later at a different time, the off-track error was much smaller.

Normally, when the VCCG experienced a sustained (greater than three computer cycles) telemetry error, network error, compass error, or GPS error, the vehicle would be commanded to pause. The operator could command it to resume when the error cleared. During part of the testing, some error-protection features were disabled to evaluate the effect of the errors on the tracking performance. Depending on the type and length of time that an error state existed, significant off-track errors could occur. Once the error was cleared, the VCCG was able to quickly guide the vehicle back to the planned path. On one occasion, the GPS was lost for a significant period of time (several seconds). This caused the vehicle to drift away gradually but returned to the planned path as soon as the GPS was healthy again (see the trajectory between point 9 and 10 in Figure 39). During this time the path tracking was under the control of the dead reckoning system.

The Compass had a bias that varied with the orientation of the vehicle. That bias was corrected by adding a calibration function in the navigation module of the on-board computer. Another problem with the compass was the sensor outputs were very oscillatory. It was later found to be mainly caused by the inclinometer in the compass. Clipping the inclinometer error threshold from 20 degrees to 10 degrees, and activating the digital filter built into the compass helped to alleviate the oscillatory behavior of the compass. The compass continued to send frequent error messages because the inclinometer is not designed for the high vibration environment that the SGTV presents. Most of the error messages were false alarms and had no effect on tracking. 


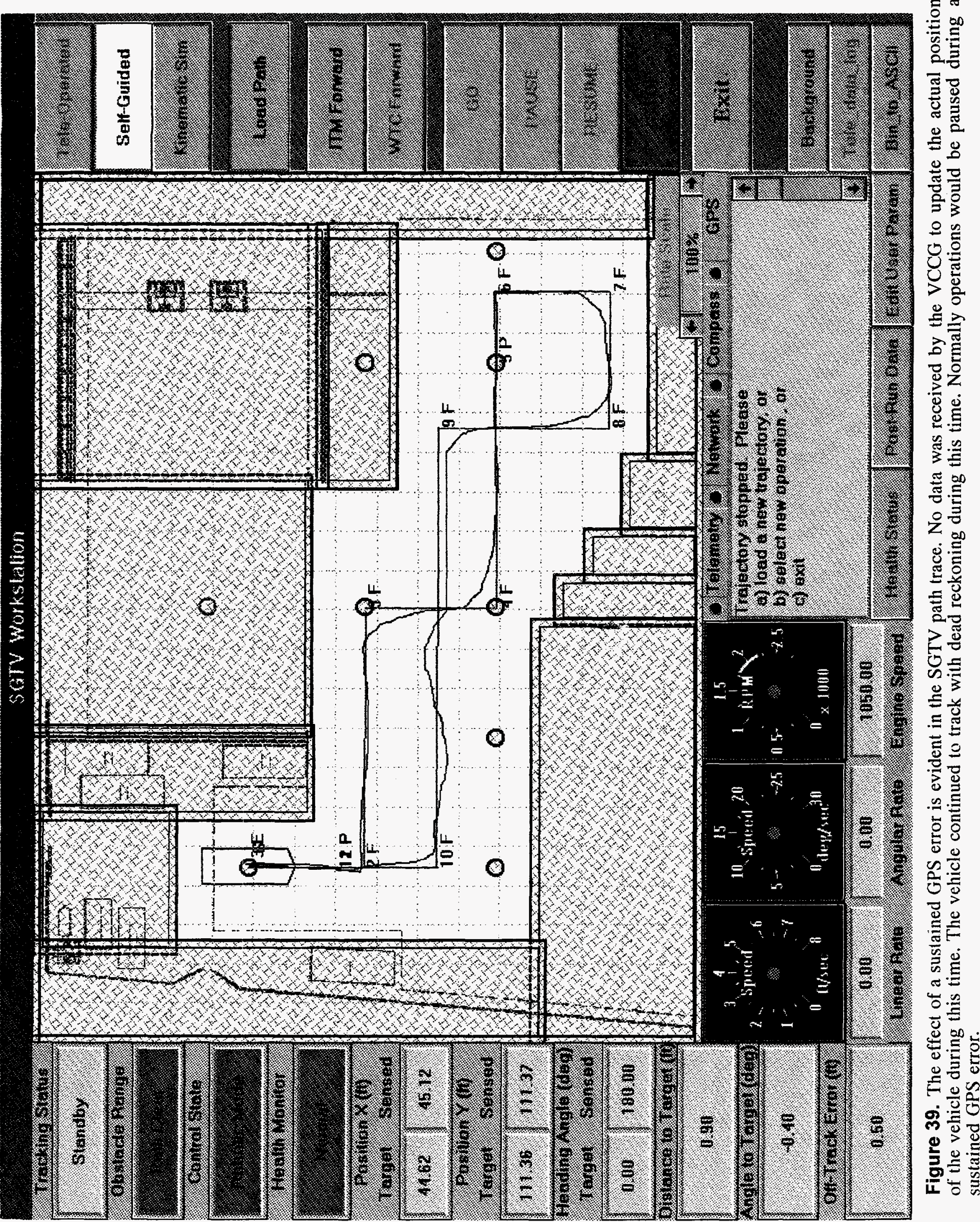


Generally, the compass error messages were ignored unless they were sustained. The compass gave very accurate readings when the vehicle was stationary. While the vehicle was moving, the compass contributed a portion of the heading estimate.

The most severe constraint on SGTV operations were due to vibration-induced network dropouts when the linear rate command exceeded $2.5-3 \mathrm{ft} / \mathrm{s}$.

Typical runs of path 1,2, and 3 are shown in Figures 40, 41, and 42, respectively. The off-track error and command and feedback rates as captured by the VCCG are shown in Figures 43,44 , and 45 .

Failure protection (e-stop functions) - In addition to the failure protection provided by the onboard controllers and the manually operated RF emergency stop device, the VCCG also has a failure protection system. The protection system takes inputs from the onboard computer and determines if the vehicle needs to stop. For example, the vehicle will be paused when the VCCG encounters a telemetry error, network error, GPS error, compass error, or an off-track error that exceed a specified limit. The VCCG can also send a hydraulic-off e-stop command to cut hydraulic power. Such command would be issued when the vehicle enters the soft stop buffer near the borders of its operating envelope or departs from the planned path beyond a specified limit. When encountering other noncritical errors such as battery low, the VCCG only displays the errors to warn the operator, who is responsible to determine whether an action is necessary.

\subsubsection{Human Factors}

In designing the MP and VCCG systems, human factors were considered in selecting the monitor, control input device, layout of the screen, colors, sizes and locations of all the buttons and boxes on the screen. All of the people who have used the MP and VCCG found them easy to learn and use. No significant problems have been encountered in either the MP or VCCG interfaces. The VCCG is extremely convenient and useful for data collection.

The feature that allows the operator to switch to another mode of operation, then back to self-guided mode without reloading the planned path proved extremely useful. It allows the vehicle to perform another task or correct position and heading errors, then complete the remaining path. Without this feature, operations are more cumbersome and changes or unexpected events become difficult to manage.

Tests were performed to compare the performance of the self-guided mode and the teleoperated mode. As expected, the self-guided mode provides much better precision and accuracy than in teleoperated mode. Even with a skilled operator, it is extremely difficult to achieve an off-track error within $1 \mathrm{ft}$ of the desired path (see Figure 46). Teleoperations require a significant amount of cognitive attention by the operator to provide rate commands to navigate, monitor on-board and external video views, as well as monitor performance, health, and OAS status, whereas the self-guided mode only requires the operator to monitor the health status. As a result, teleoperations are significantly more stressful on the operator than self-guided operations. The teleoperated mode relies on cameras to provide feedback to the operator, and therefore can only be operated with sufficient lighting on the worksite. On the other hand, the self-guided mode, with all the health monitoring and safety systems fully functional, can operate in total darkness. 


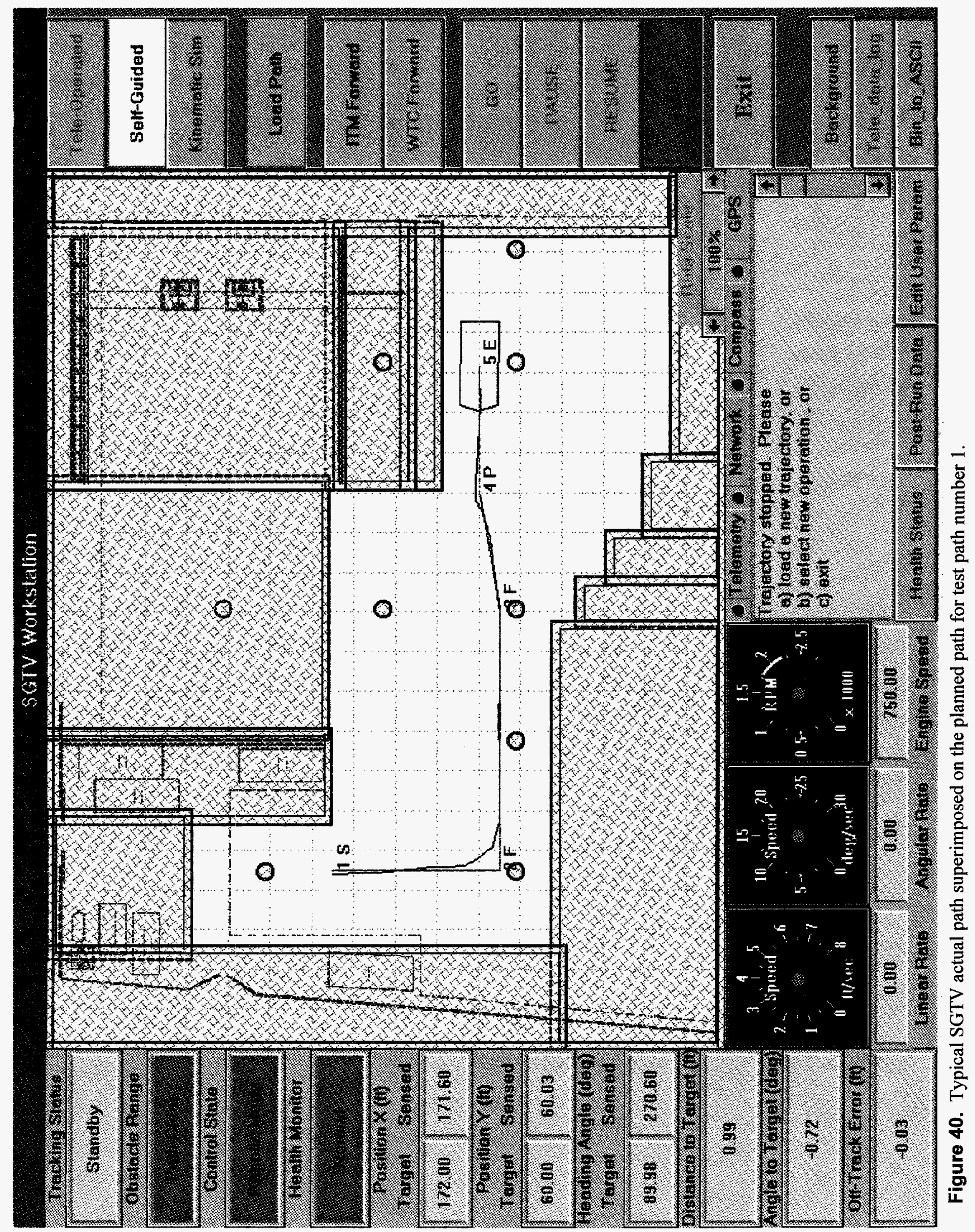




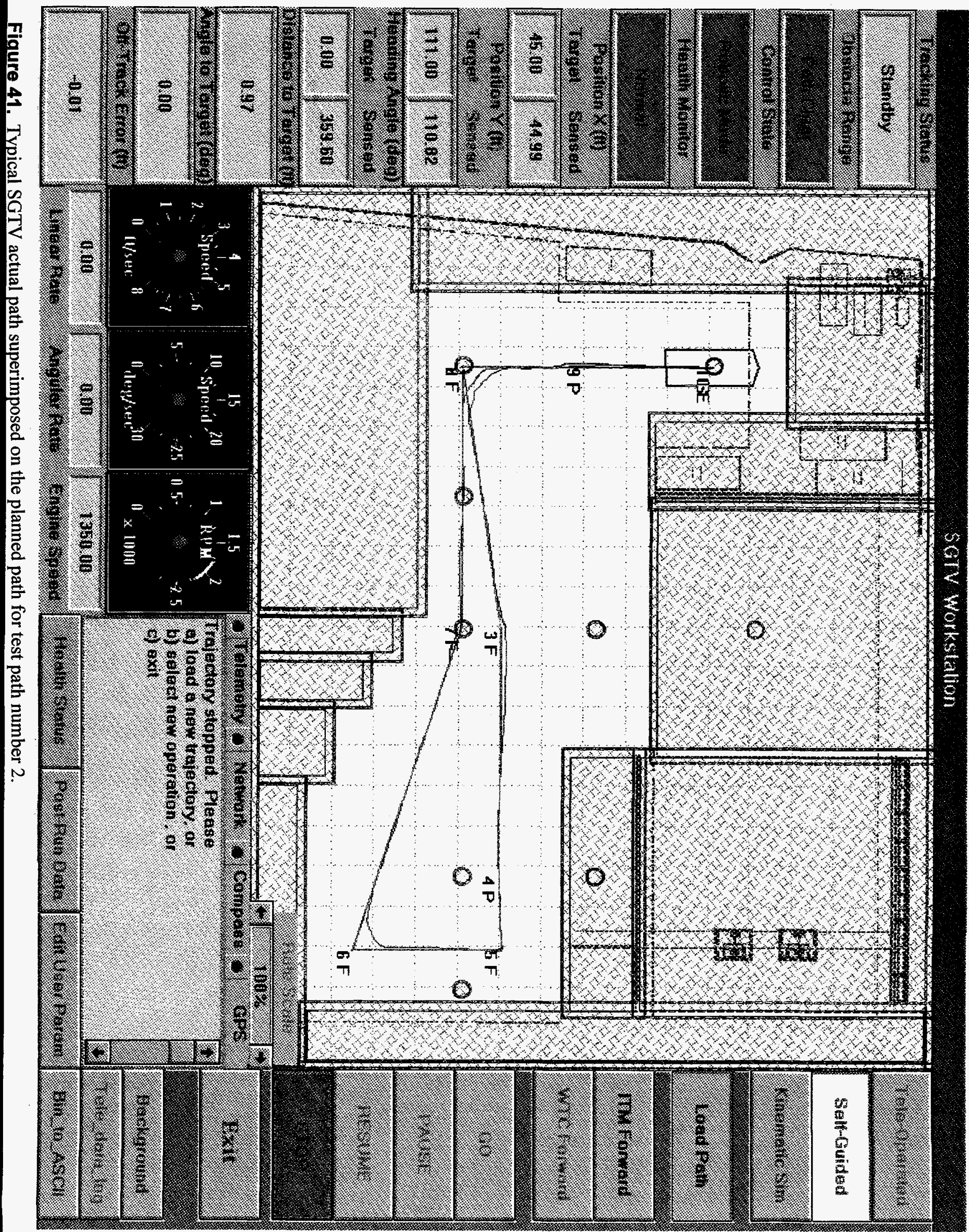




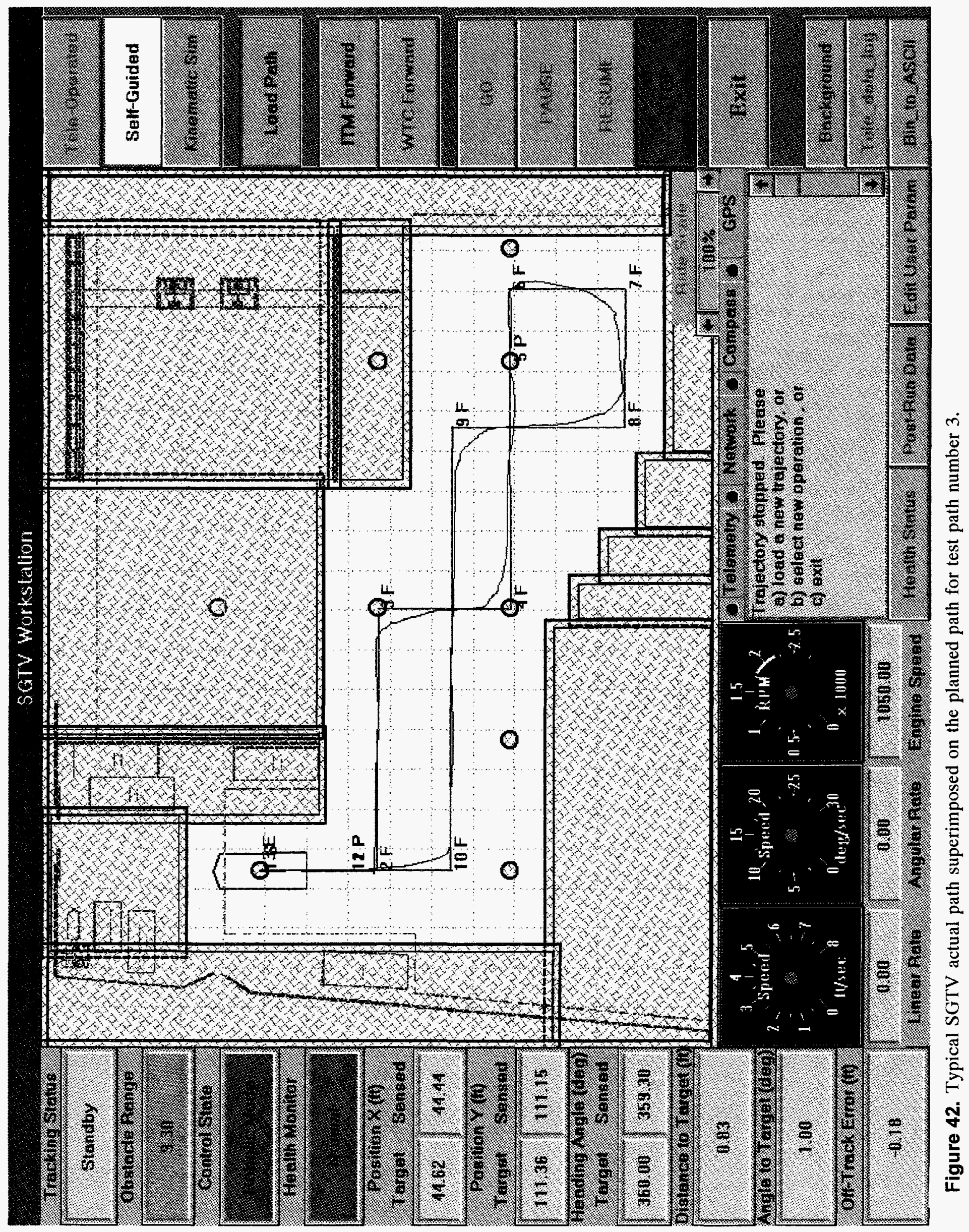




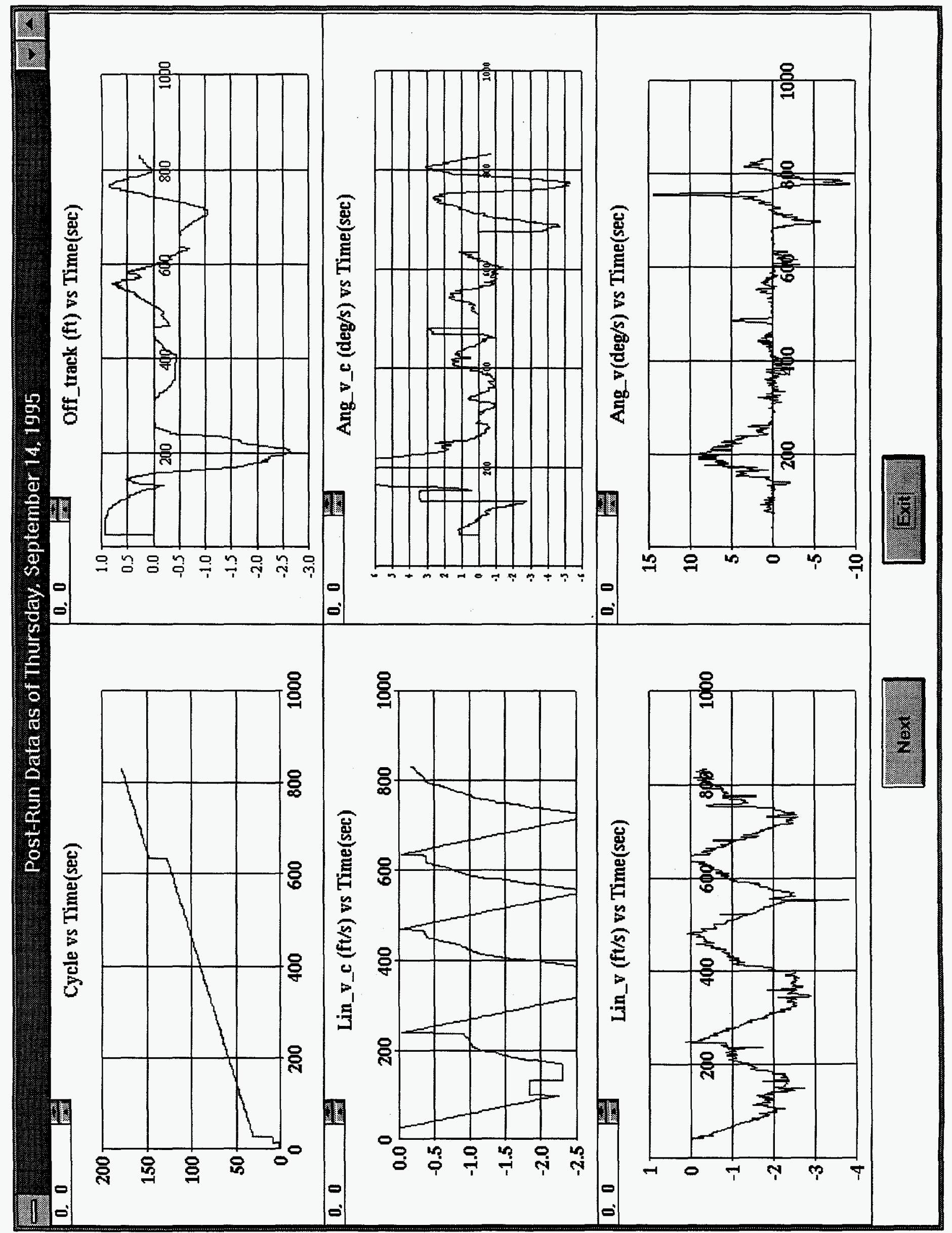




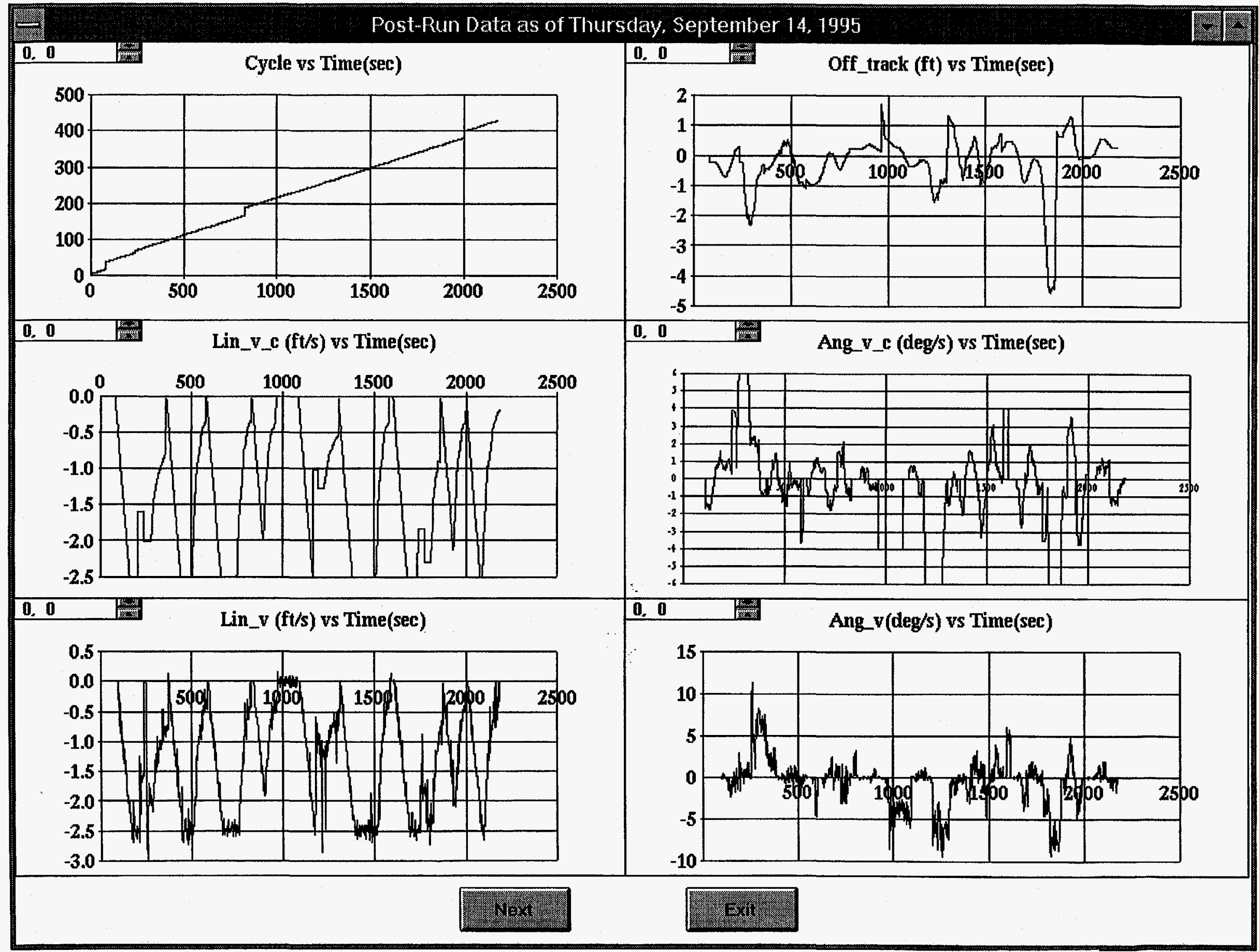

Figure 44. Sample VCCG data screen showing off-track error, command signals, and SGTV response for test path number 2. 


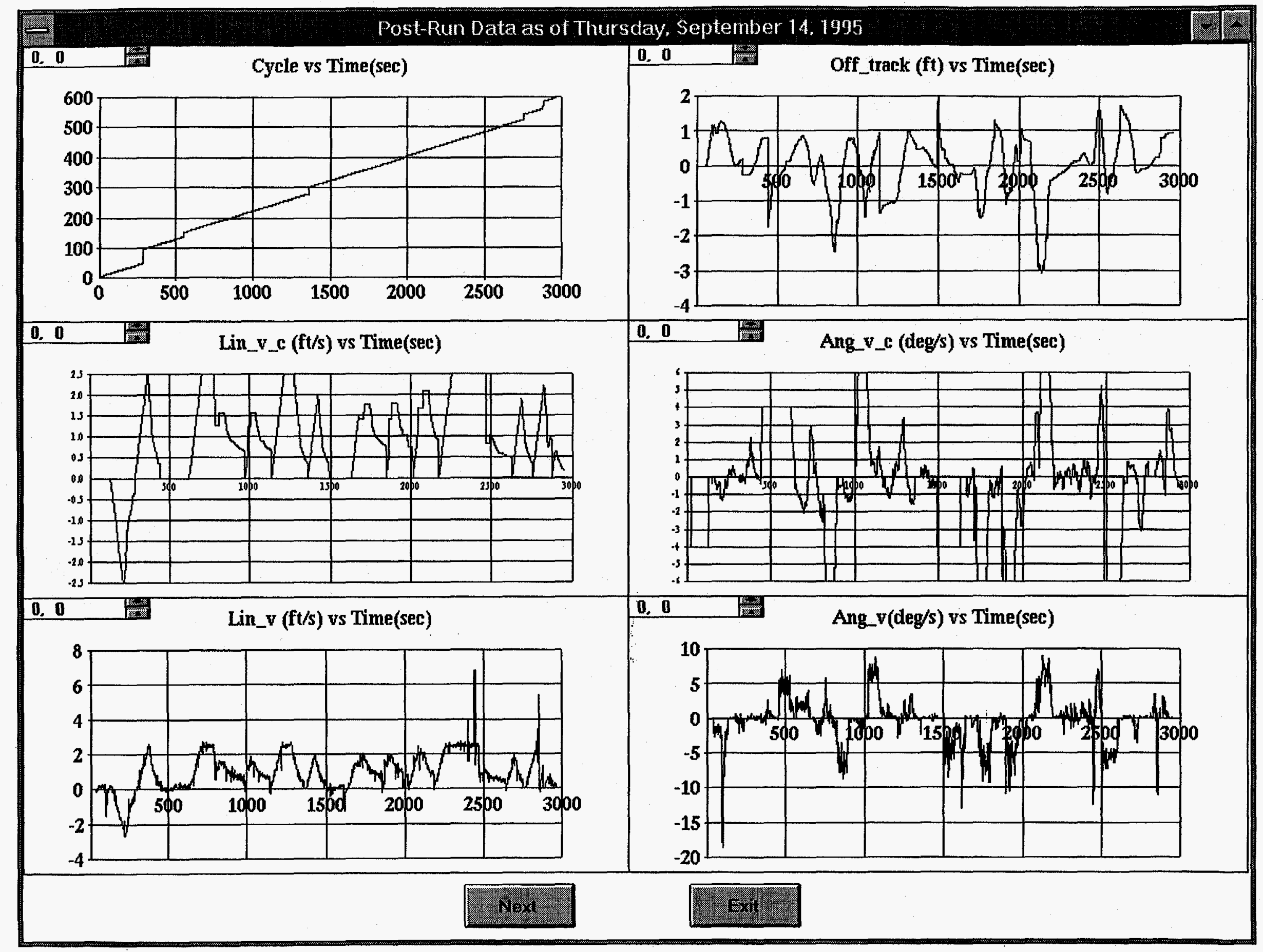

Figure 45. Sample VCCG data screen showing off-track error, command signals, and SGTV response for test path number 3. 


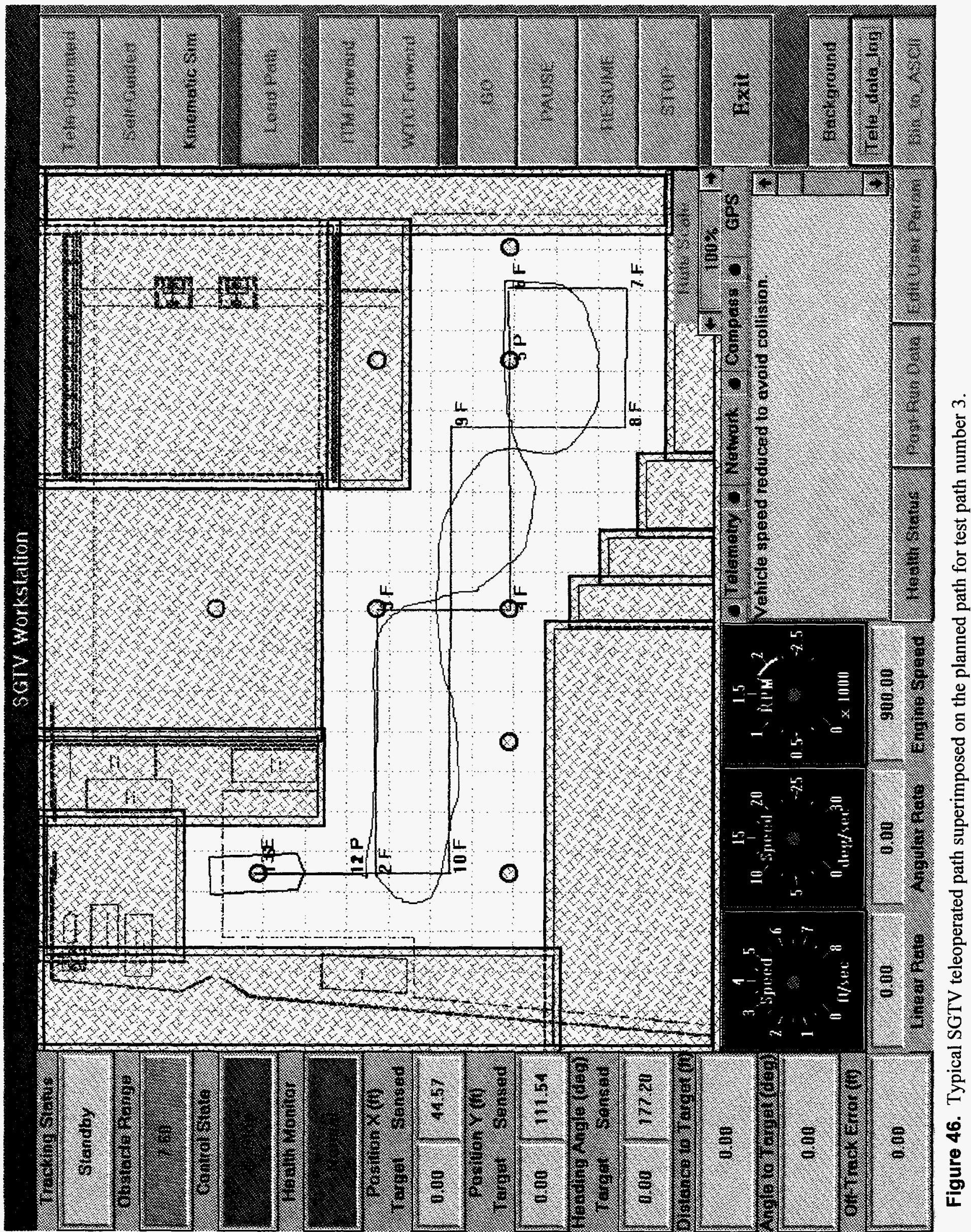




\subsubsection{Recommended Improvements and Future Work}

- At this time, the collision detection function of the MP treats all the flyby points as a pure rotation flyby. As such, the collision detection results will be very conservative at smooth flyby points. To improve it, the MP software should integrate the same smooth flyby algorithm as in the VCCG.

- The current VCCG does not modify the linear rate commands in proportion to the off-track error. Incorporating this capability would make it easier to correct offtrack errors when the vehicle encounters unexpected disturbances such as EMI or sensor failures.

- The smooth flyby performance can be improved by balancing the feedback of offtrack error and the heading error more effectively. The technique used in the current system is to precalculate the linear command based on the radius of the circle (smooth flyby tangential circle) which has two linear path segments as its tangents. The angular rate command is calculated based on the off-track error and the heading error. The smooth flyby performance can be improved by constraining the angular rate command using: angular rate command = linear rate command divided by the radius of the smooth flyby tangential circle. To meet the constraint, the weighting of the off-track error and the heading error have to be adjusted.

- After testing the VCCG with the SGTV, we recognized that its code can be further optimized to reduce the computing time, mainly in the GUI segment. For example, the audio warning "beep" causes considerable delay; therefore it should only be used provided it does not degrade the control performance. Passwords should also to added to prevent nonauthorized use of the program. Also, a password should be added to the user parameter editor.

- Visual Basic was selected for this project because it has more features than Visual $\mathrm{C}++$ for developing a GUI. However, the latter is more efficient for mathematical computation. The optimal approach will be to convert the control algorithms to Visual $\mathrm{C}++$ and import them into the GUI software. To do this, the interface between the GUI and control algorithms would have to be reorganized.

- To improve the off-track accuracy at high-speed tracking, two techniques can be investigated. First, move the VCCG tracking software to the onboard computer system, but retain the GUI software at the workstation. Second, develop a position predictor in the VCCG to account for the time delay in the control loop.

\subsubsection{Conclusions}

The key functions of the SGTV, Mission Planning and the VCCG, were developed and tested successfully. Mission Planning is an effective and flexible tool to create and modify worksite plans. An operator can create no-go zones and multiple path plans in a very short period of time. The program is user friendly and no significant problems were encountered during the testing. The mission planning software also allows the operator to analyze the paths to determine potential operational conflicts or collisions. This software greatly enhances the SGTV's 
flexibility to quickly deploy and operate at different work sites, with minimal support infrastructure.

The VCCG software controls the vehicle following a preplanned path. The program is user friendly and provides sufficient display data and health monitor information to allow the operator to easily monitor the SGTV's status. The VCCG's robust control system is designed to be very safe and easily handle failures or errors when encountered. During the testing, when few problems (such as EMI and network errors) were encountered, the vehicle was able to achieve an off-track accuracy well within the $\pm 1 \mathrm{ft}$ goal on a very consistent basis. The headings and absolute positioning were exceptionally accurate at the pause and final points; as a result, docking required no intervention via teleoperations for precision alignment. In transient state, the off-track error varied with the initial heading error and off-track error, but always converge to the desired path smoothly and quickly. Operating procedures and methods for the VCCG GUI were smooth and logical. 


\section{REFERENCES}

1. D. A. Arrenholz, and J. L. Knight, A Brief Analysis and Description of Transuranic Wastes in the Subsurface Disposal Area of the Radioactive Management Complex at INEL, EGG-WTD-9438, EG\&G Idaho, Inc., February 1991.

2. L. C. Meyer, Transuranic Contamination Control Using Electrostatic Curtains (Proof-of-Principle Experiments), EGG-WTD-9336, EG\&G Idaho, Inc., November 1990. 
APPENDIX A

Test Run Data Graphs 



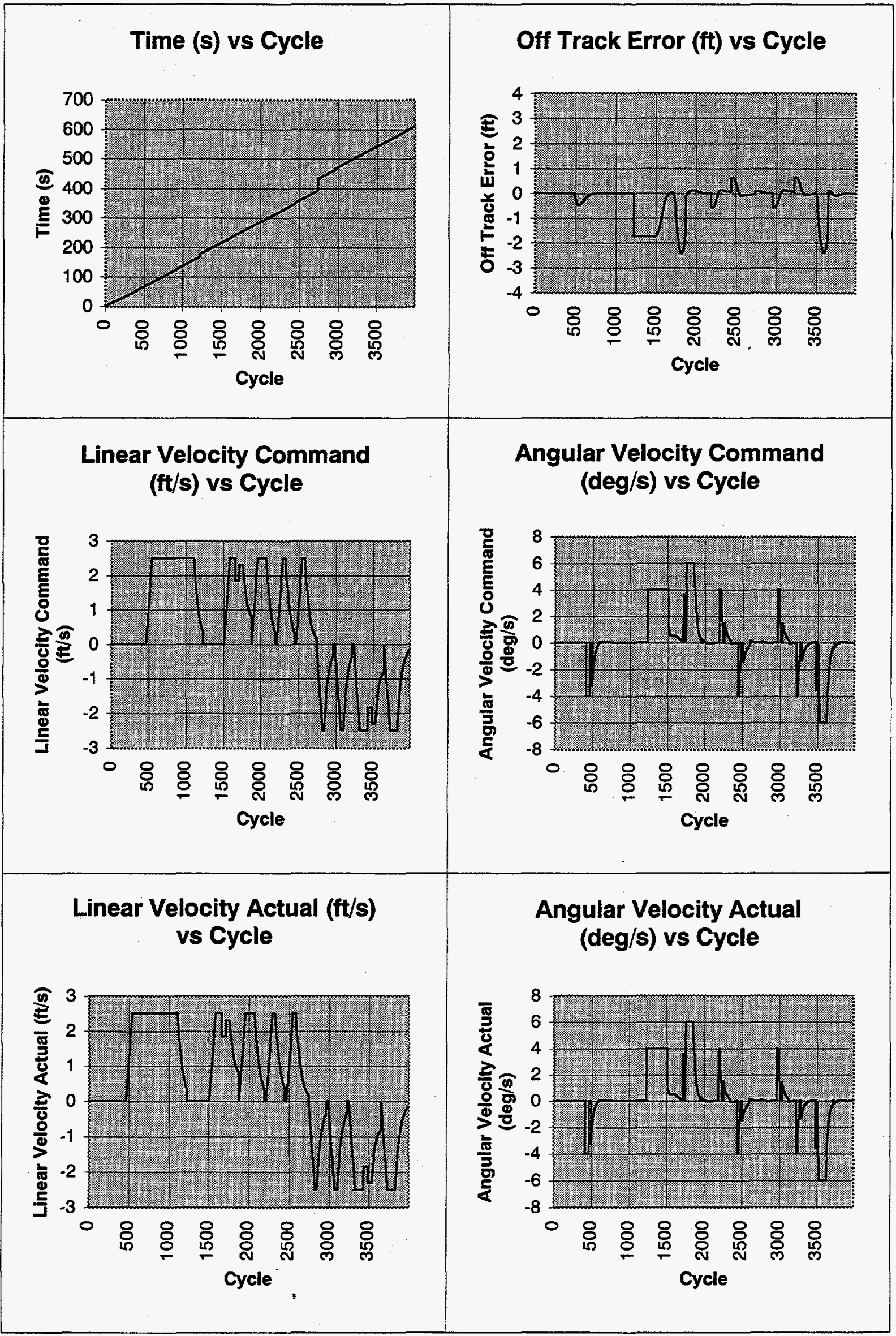




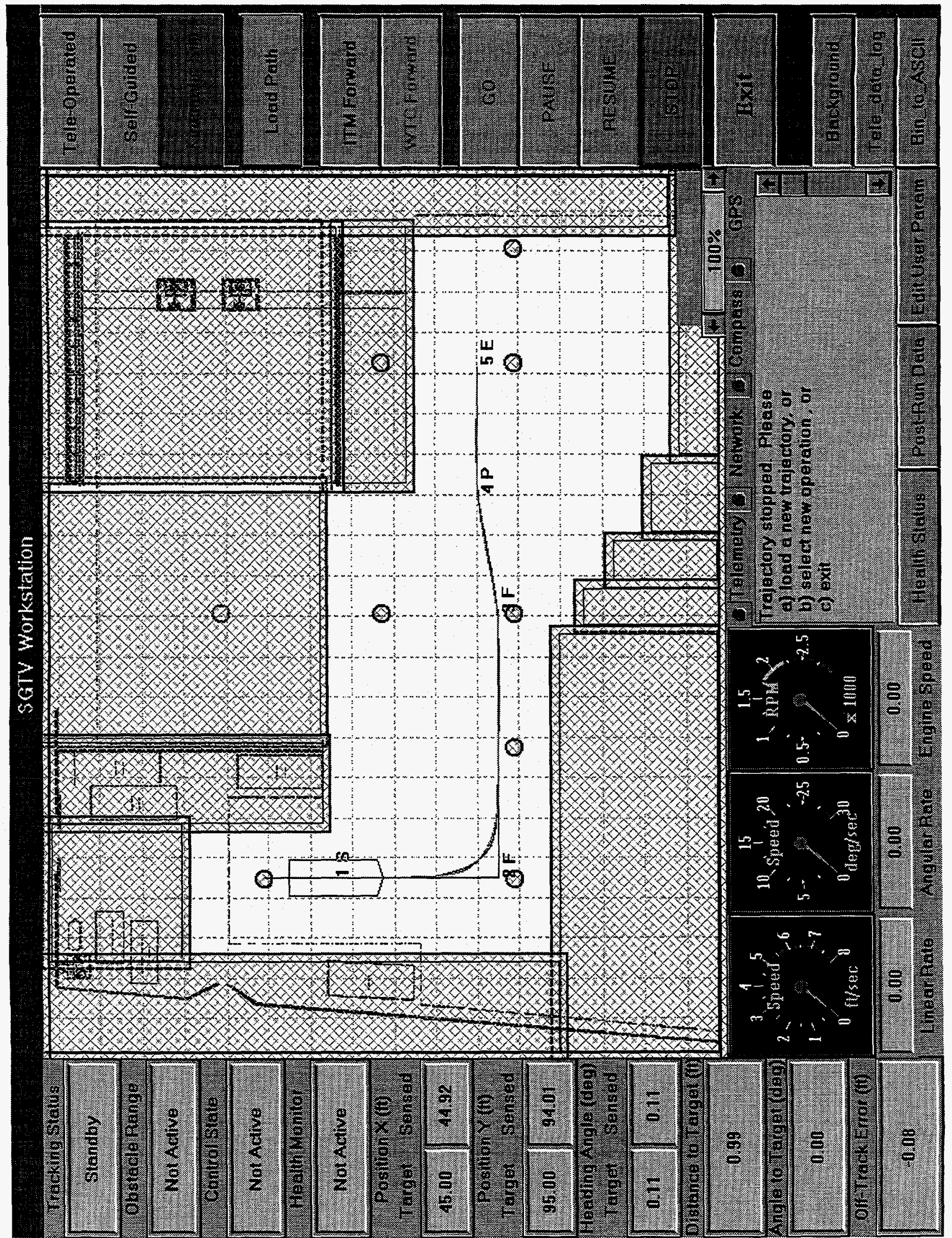




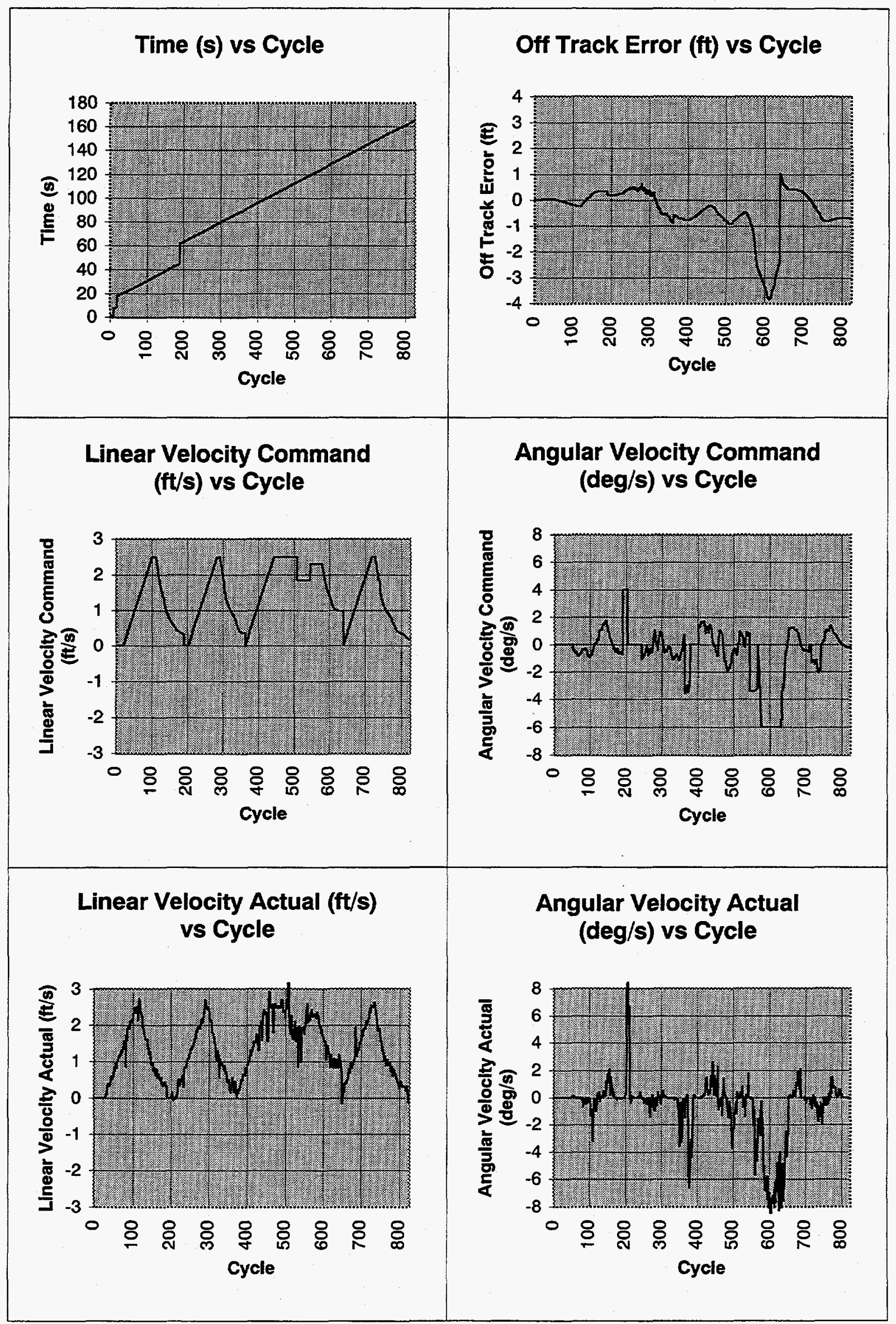


โบ โd I

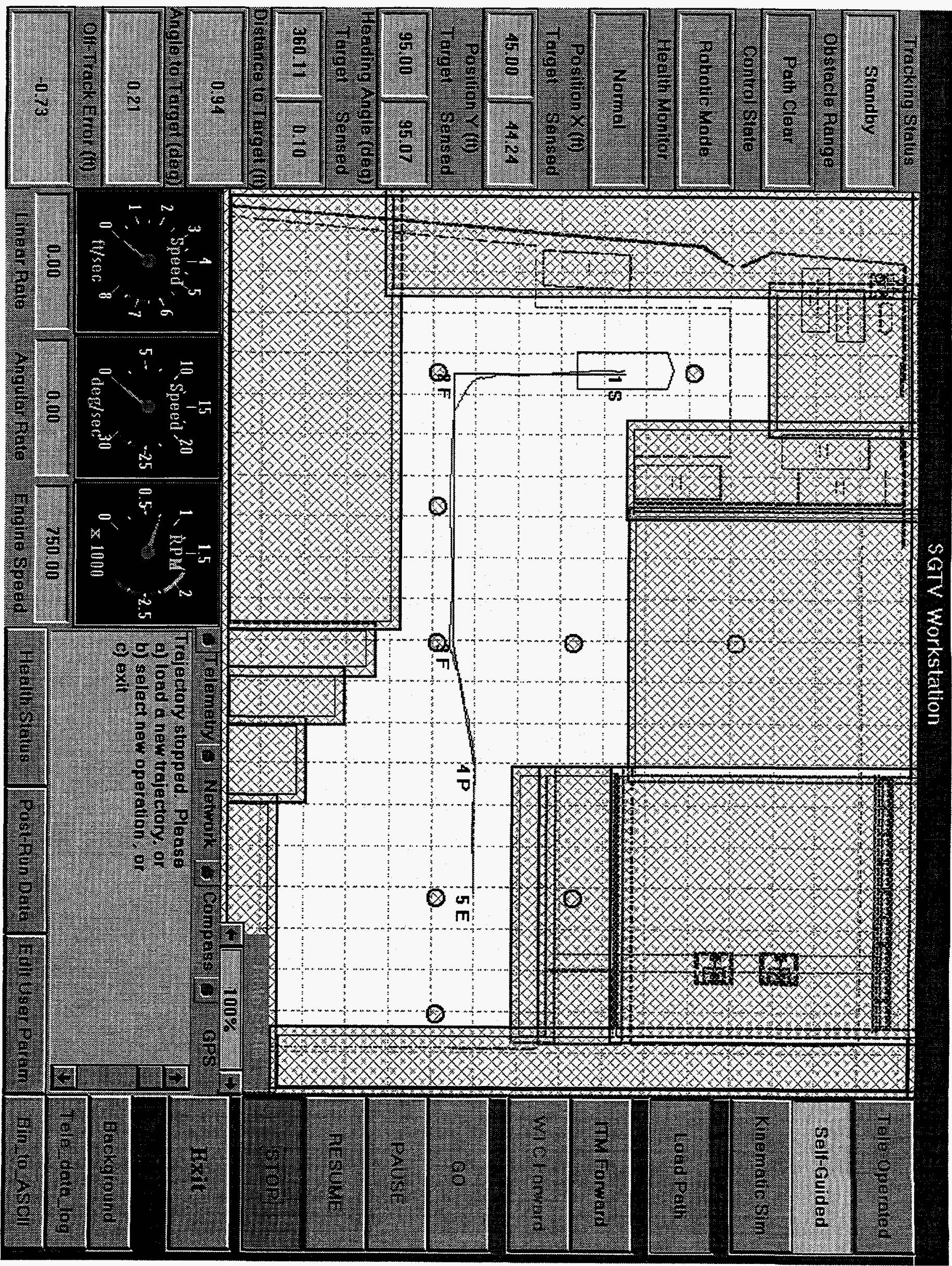




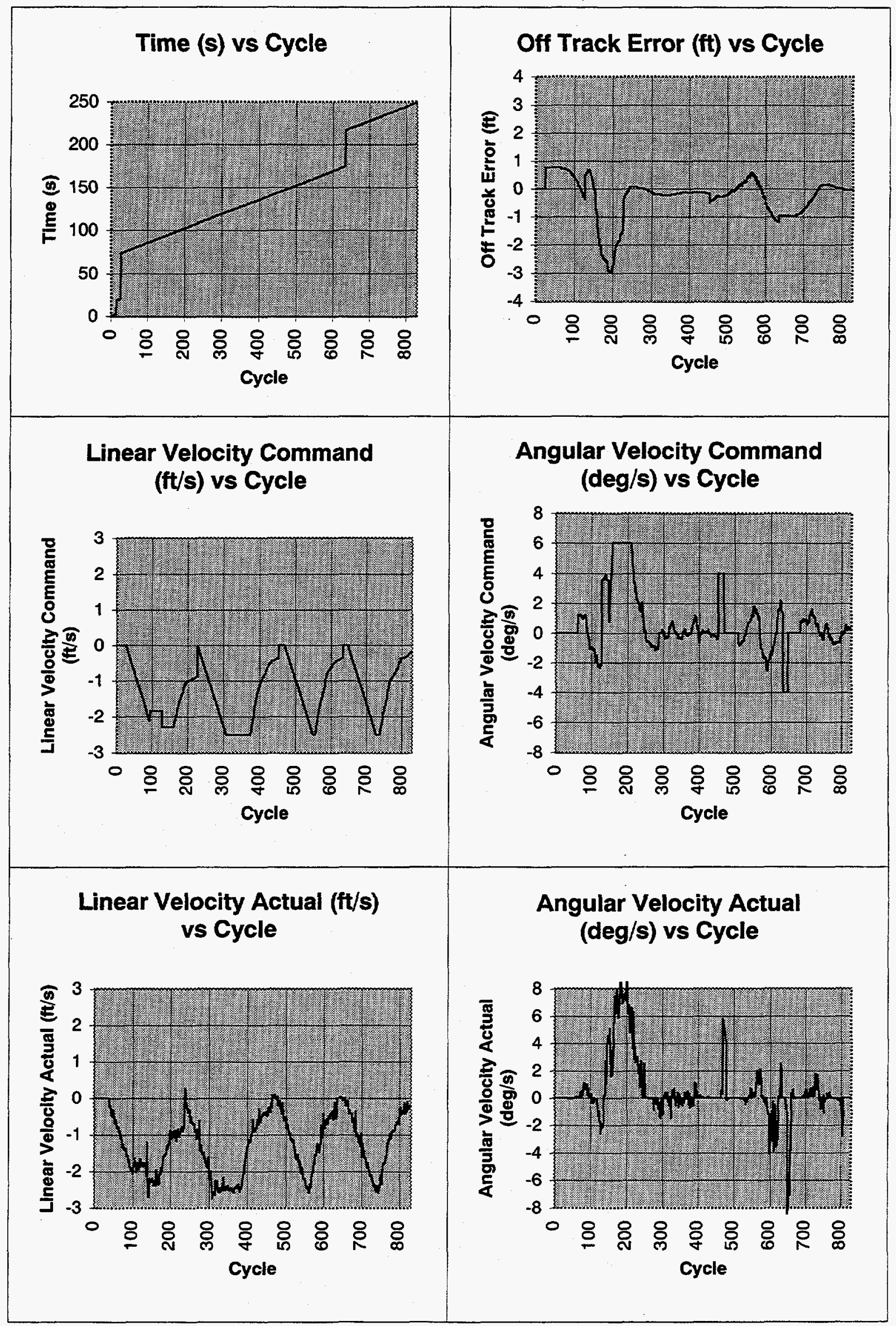


$2 y^{-} I d \perp$

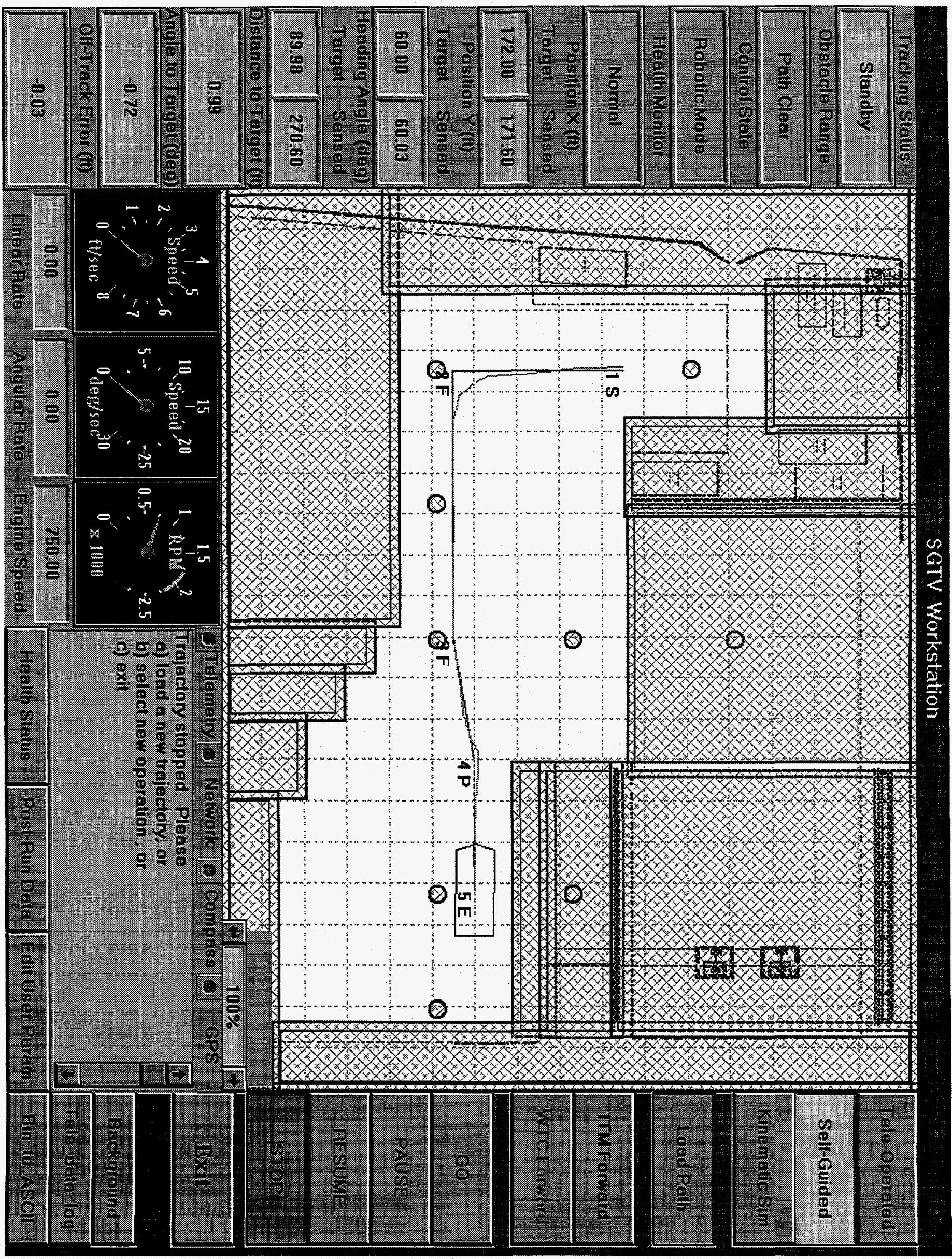




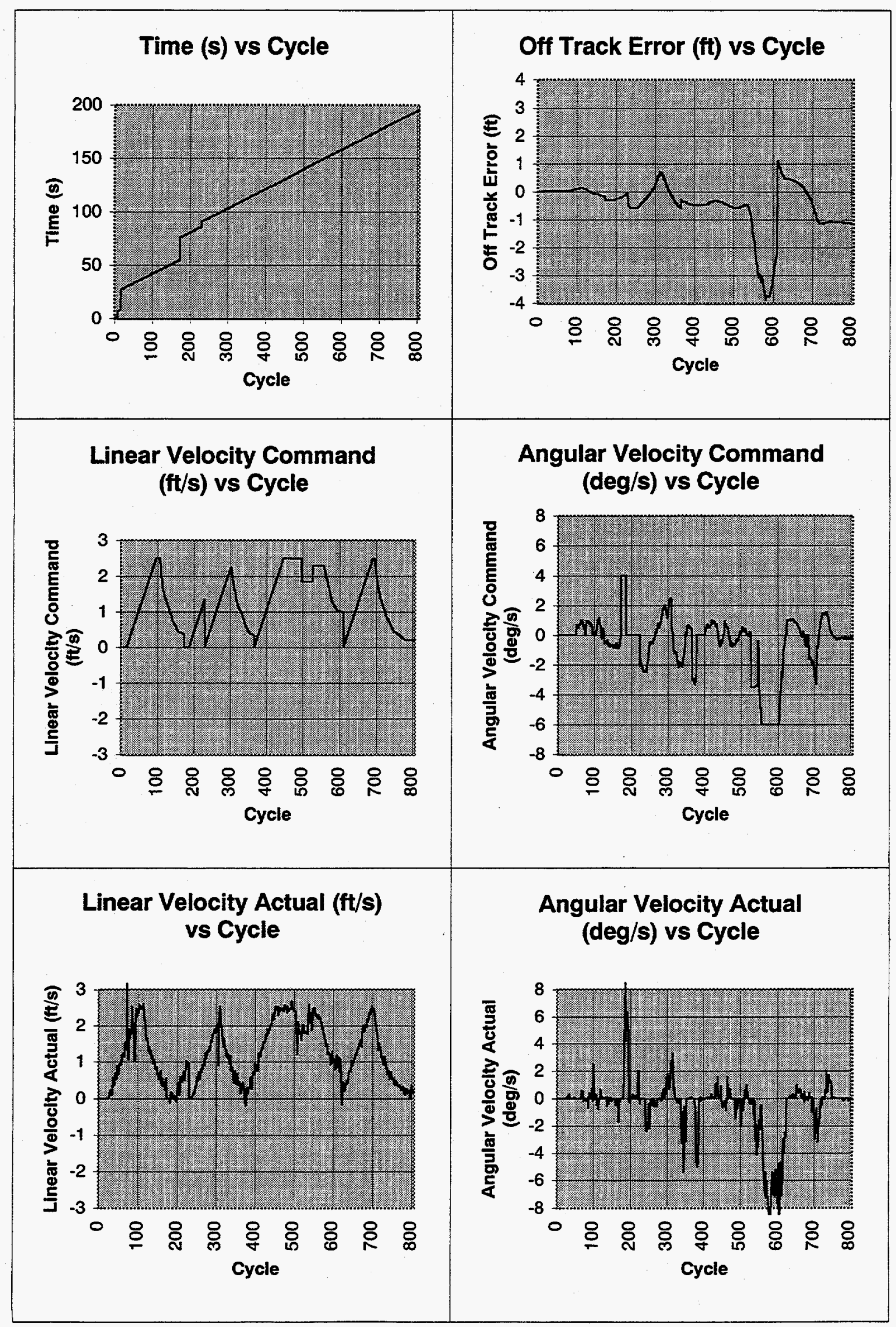




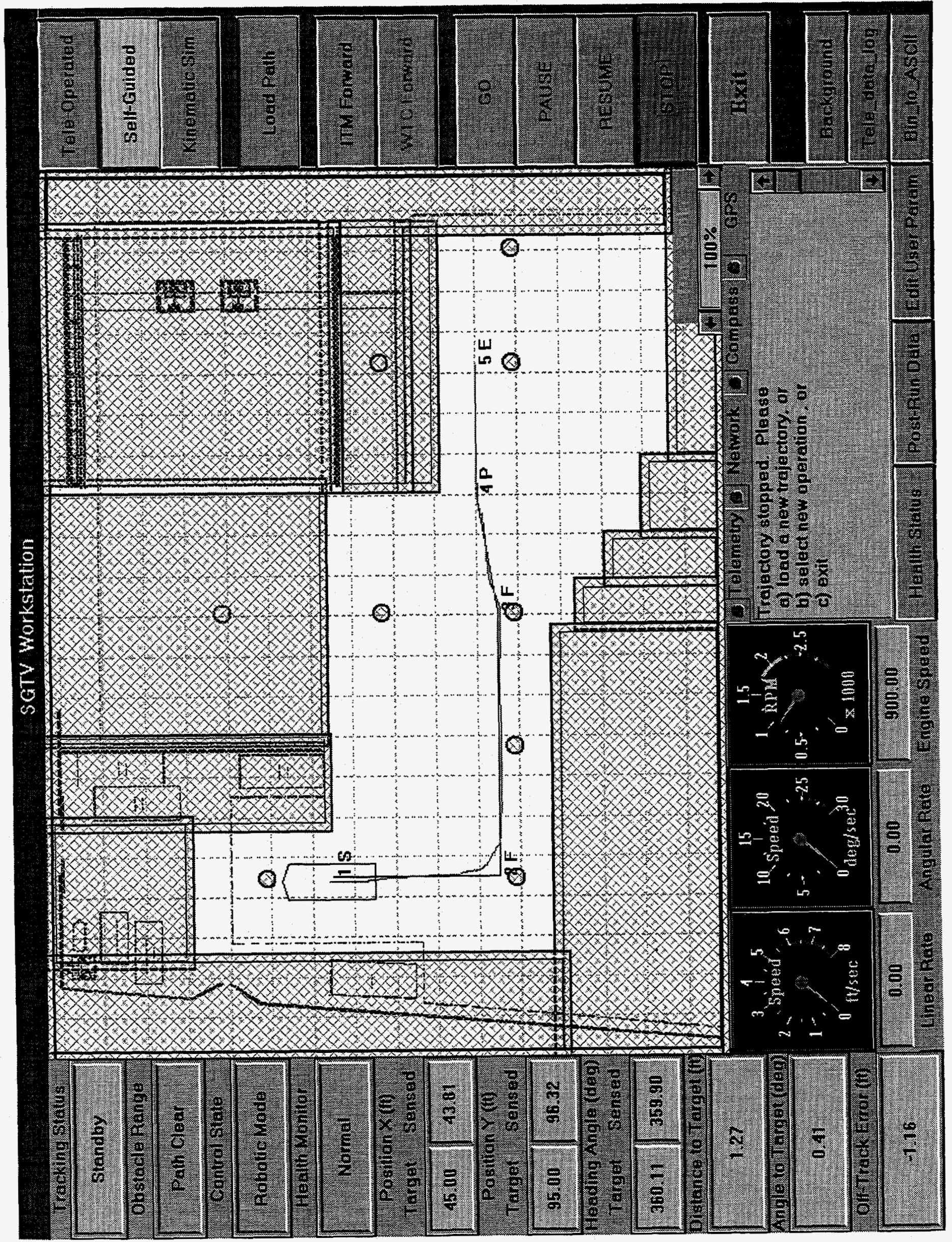




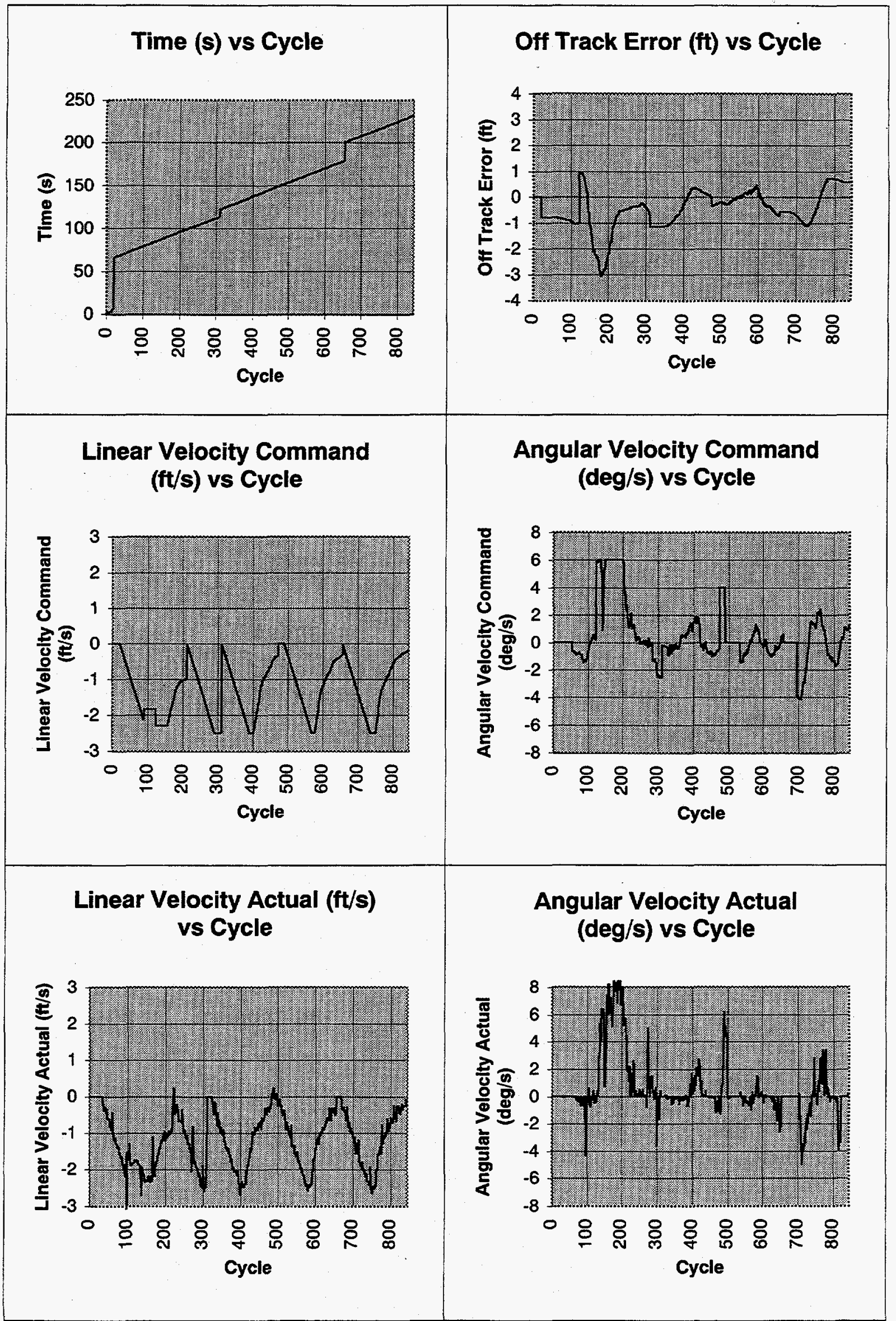




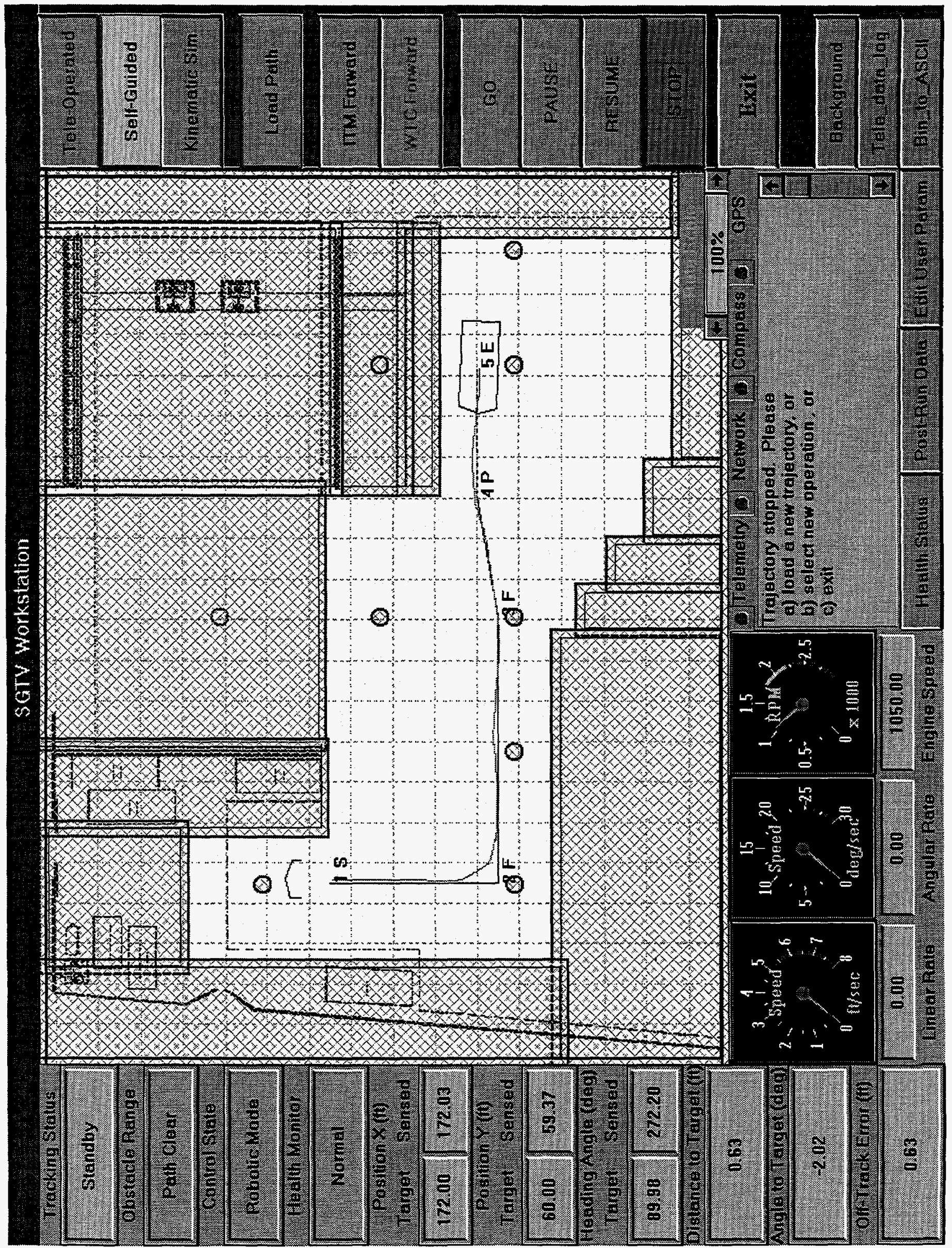




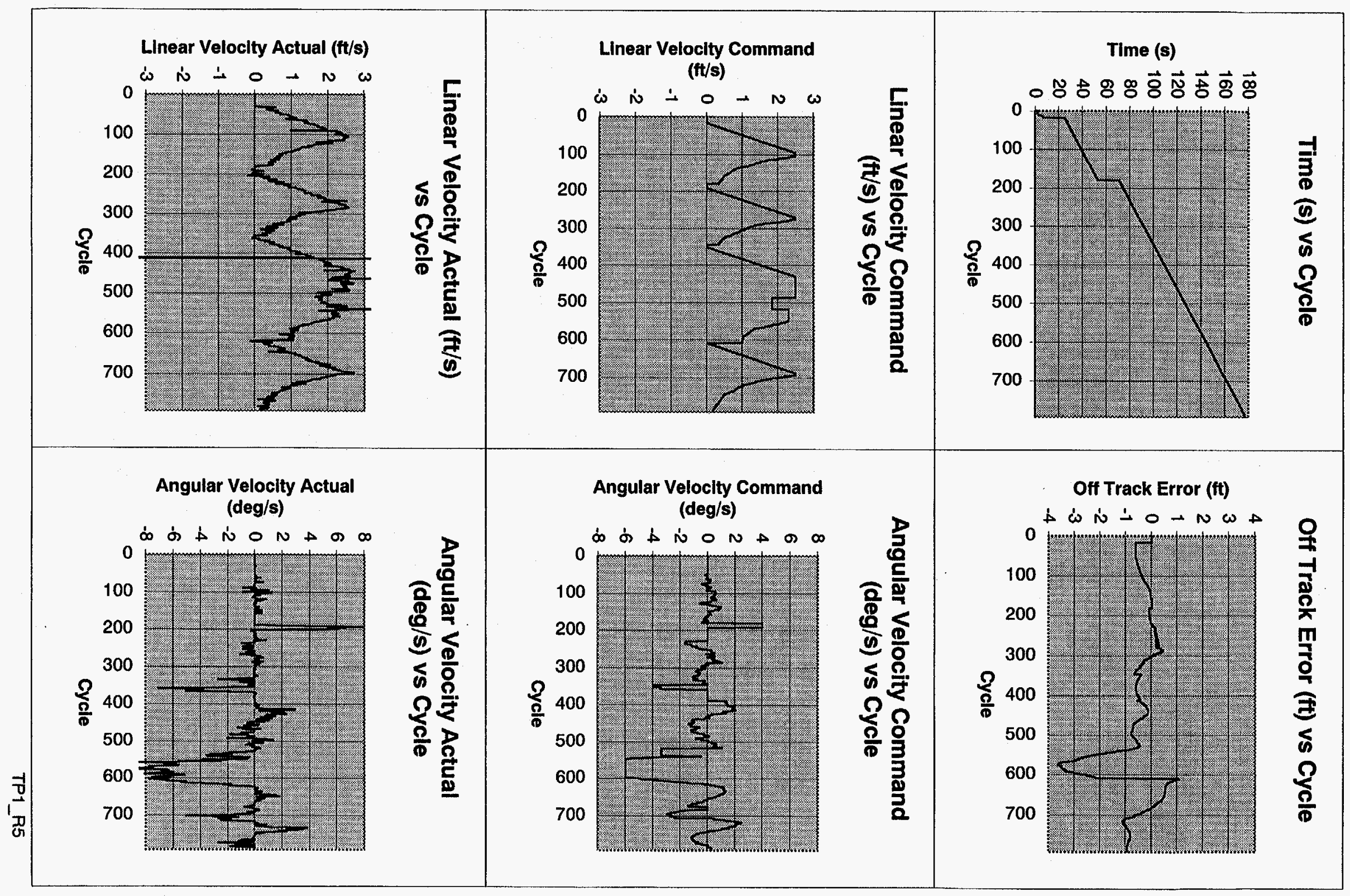




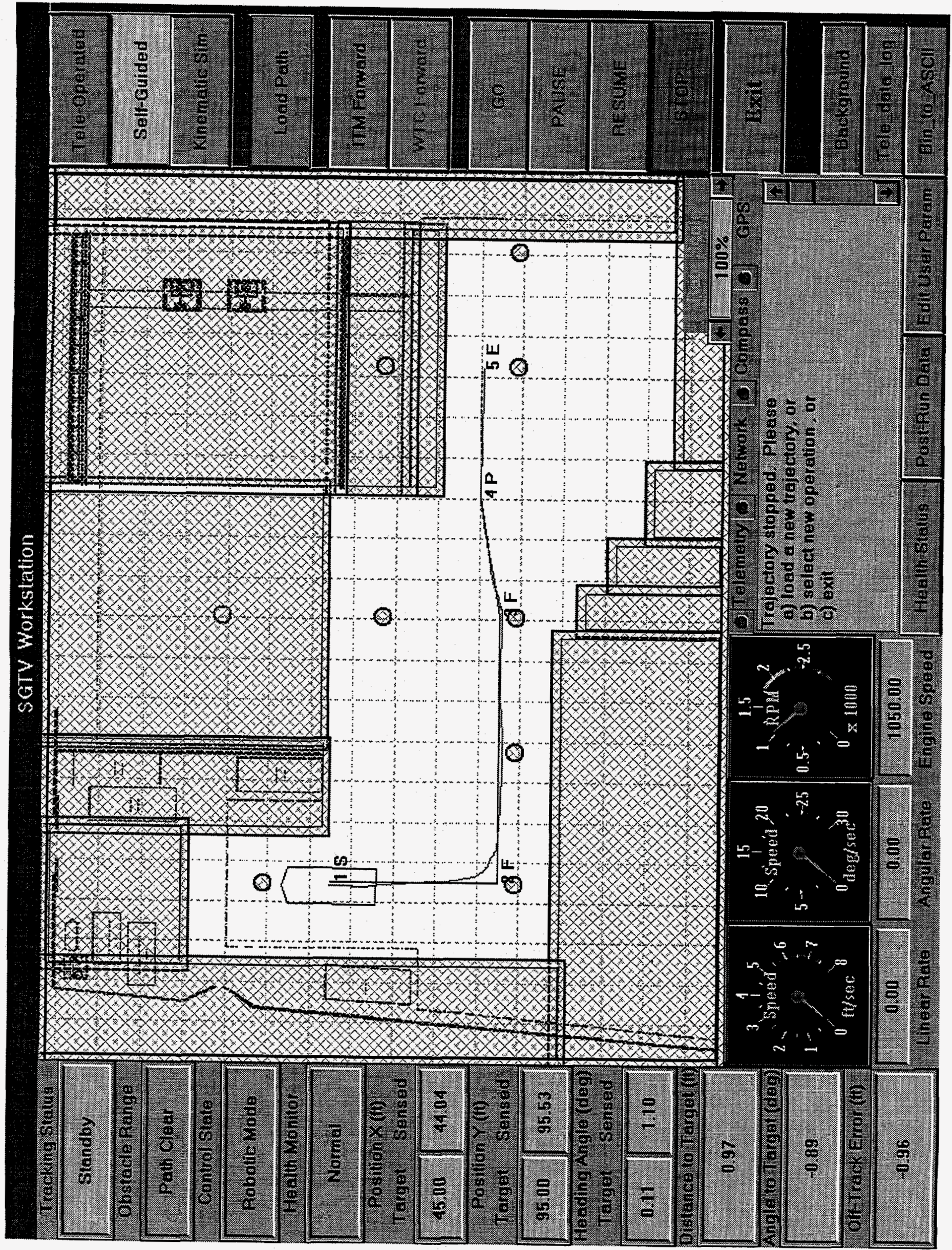




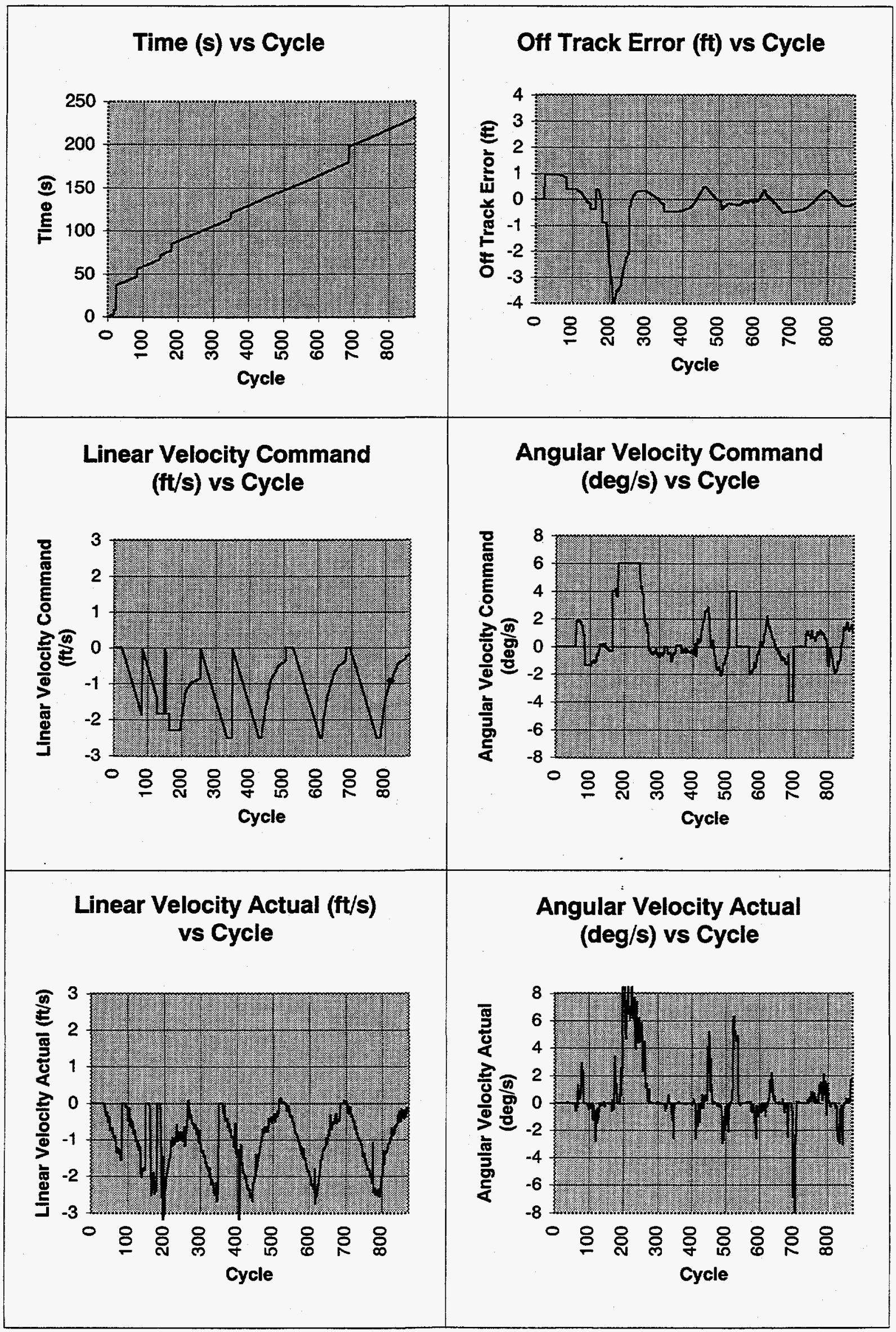




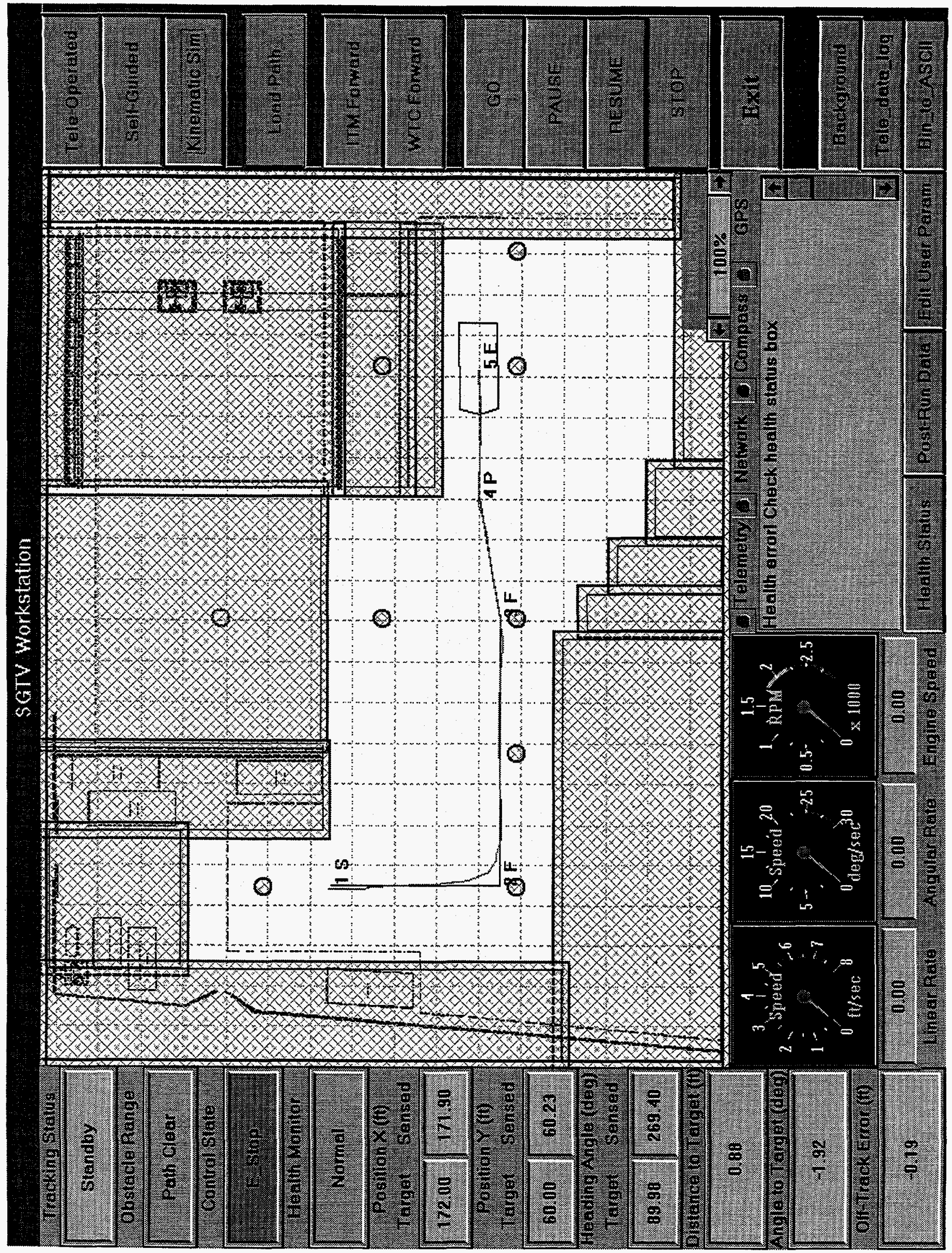




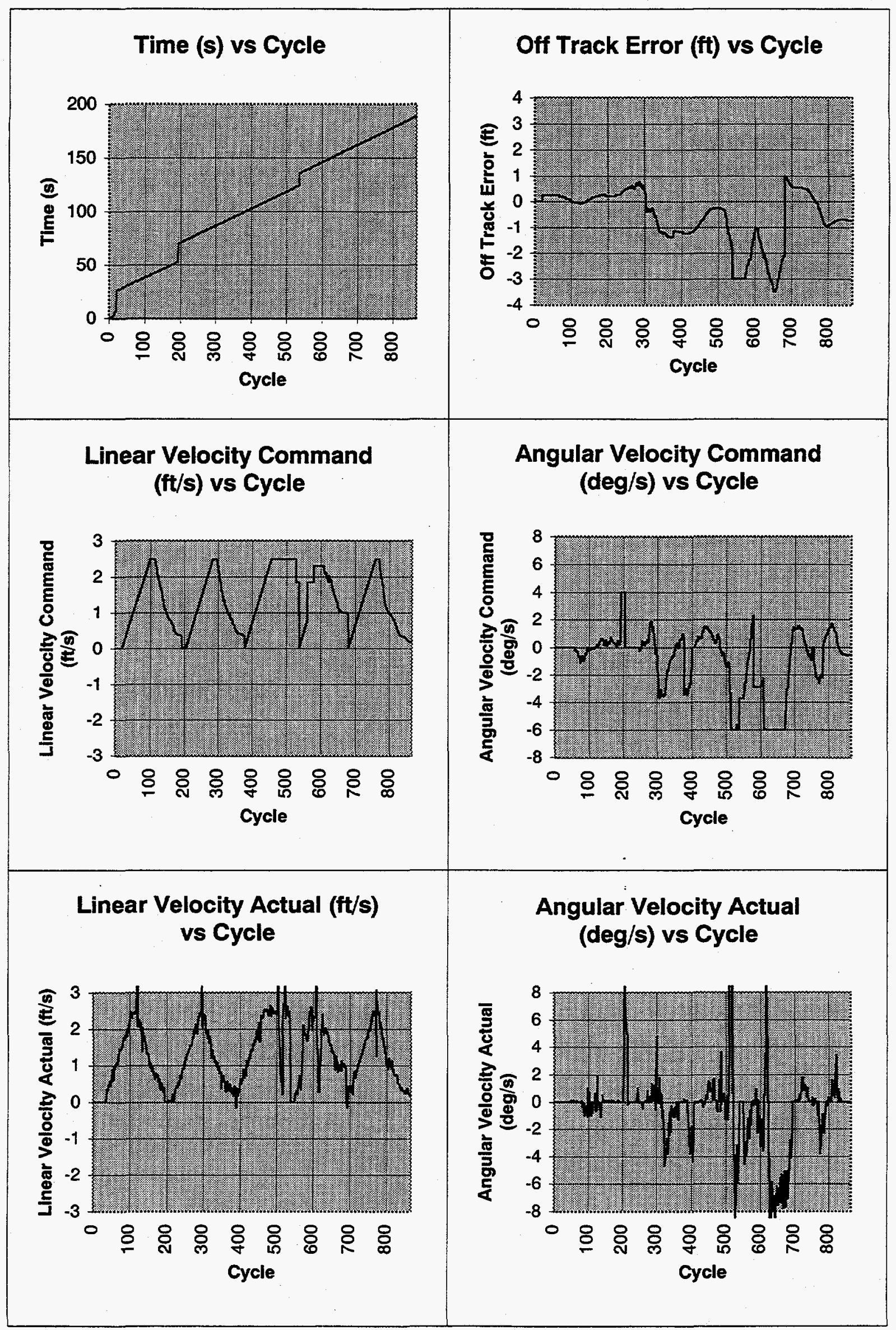




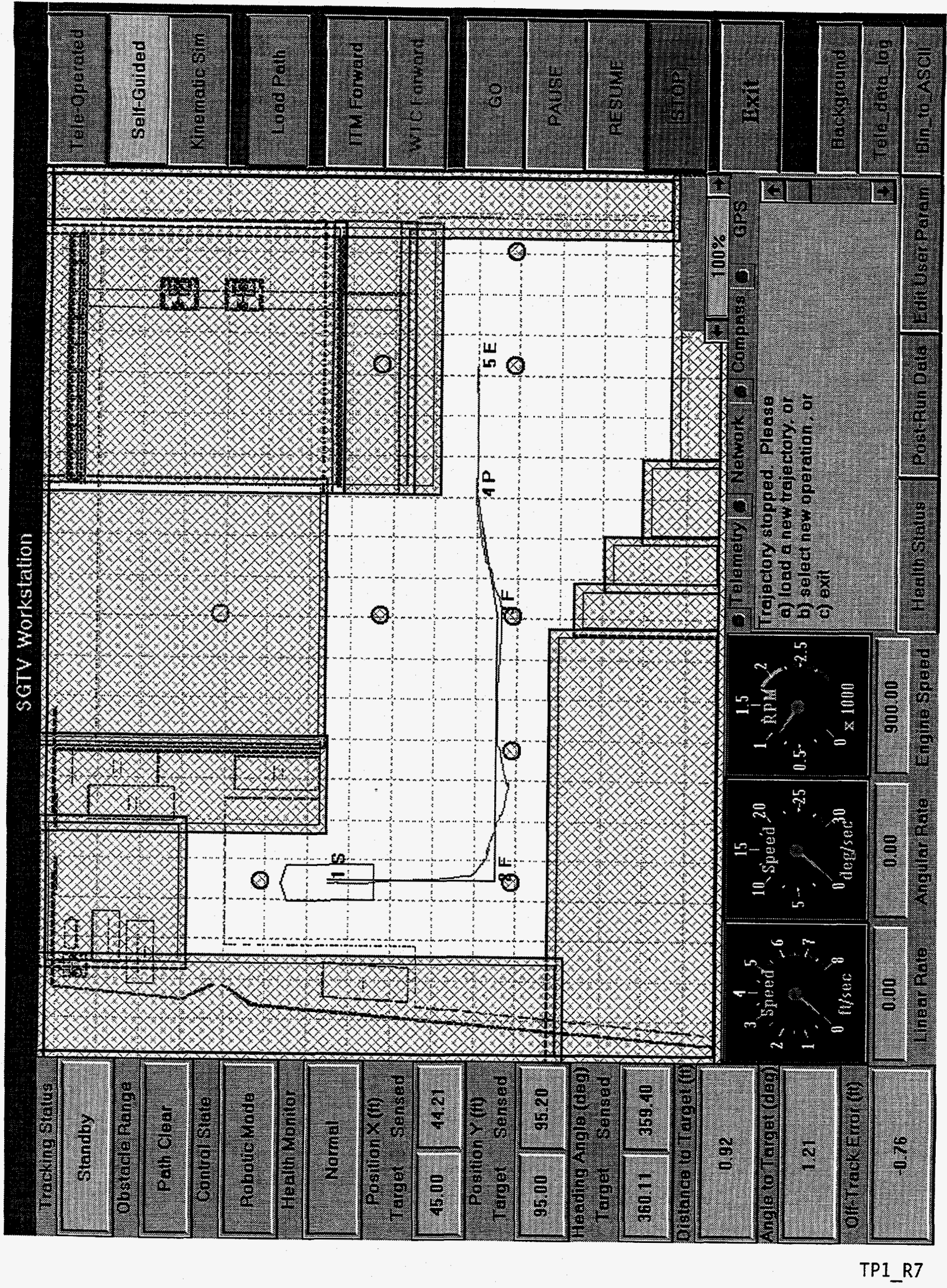




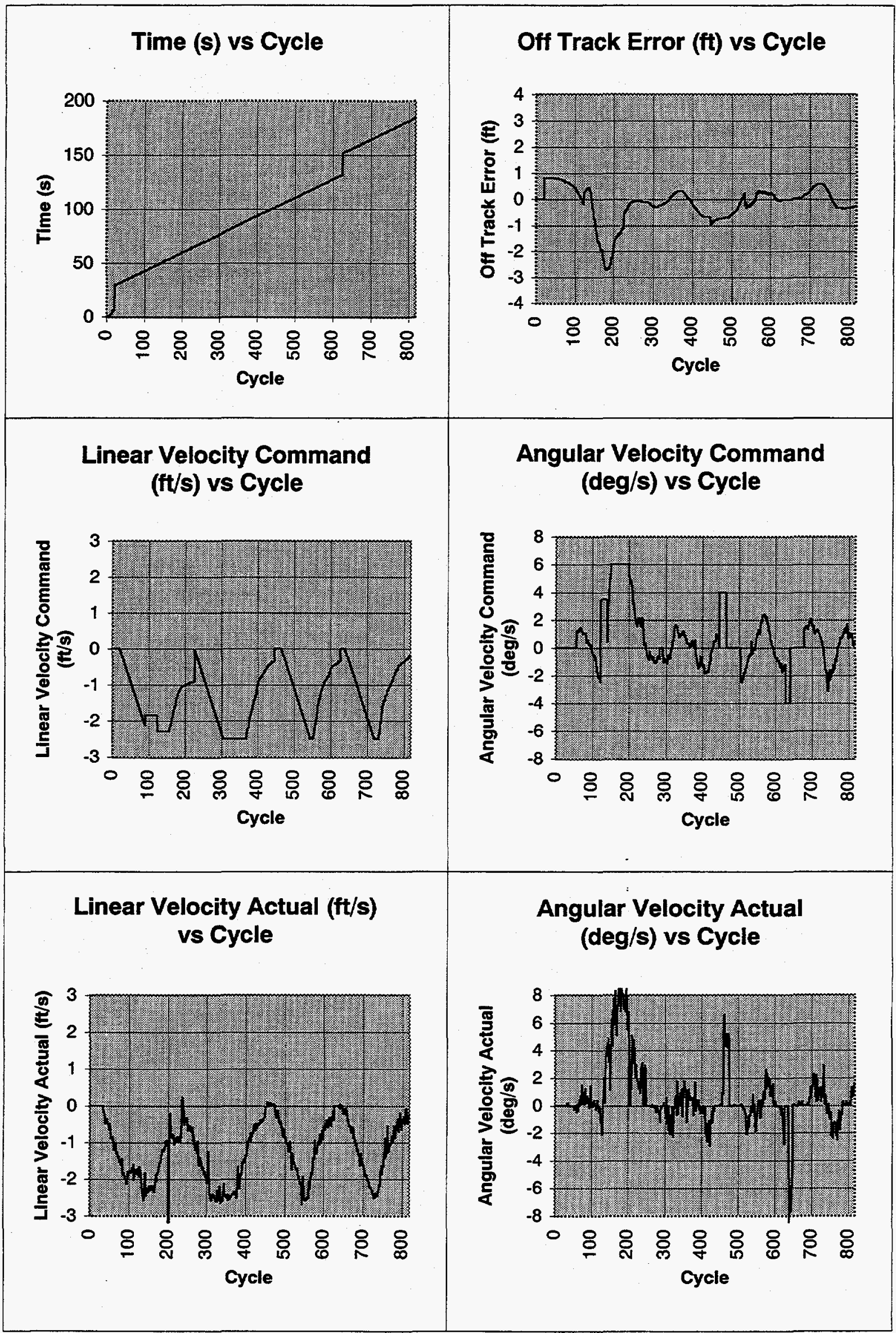




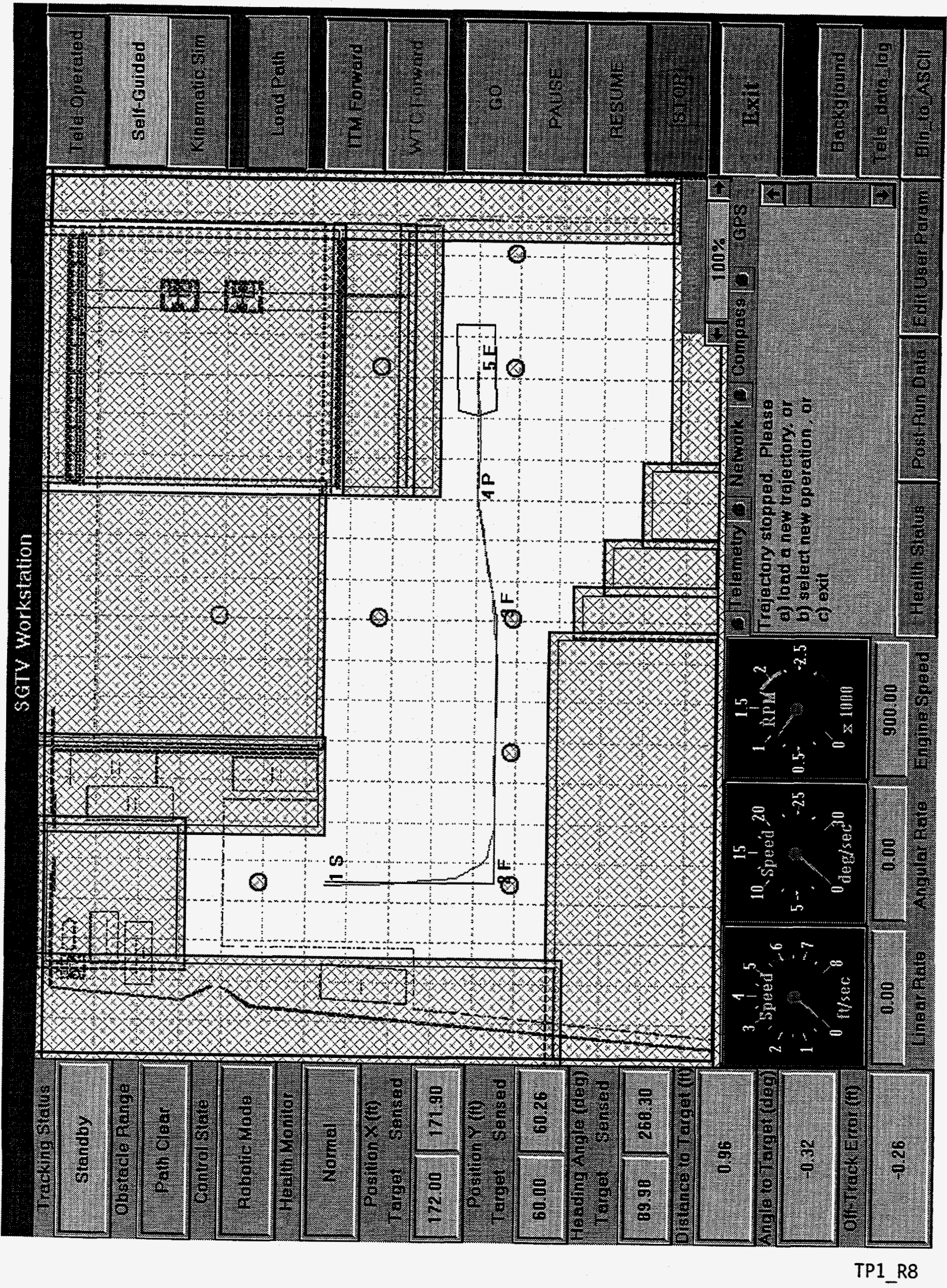




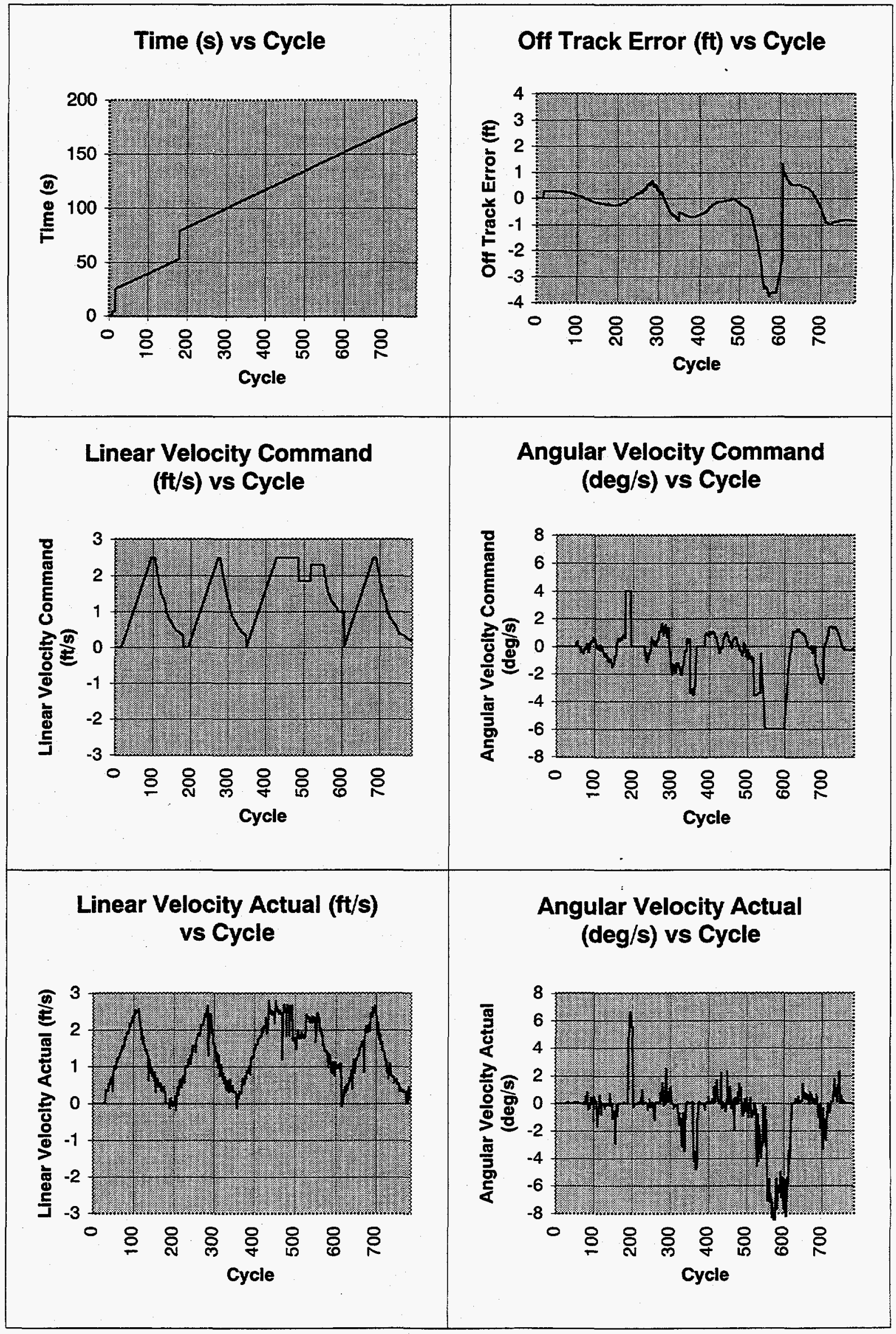




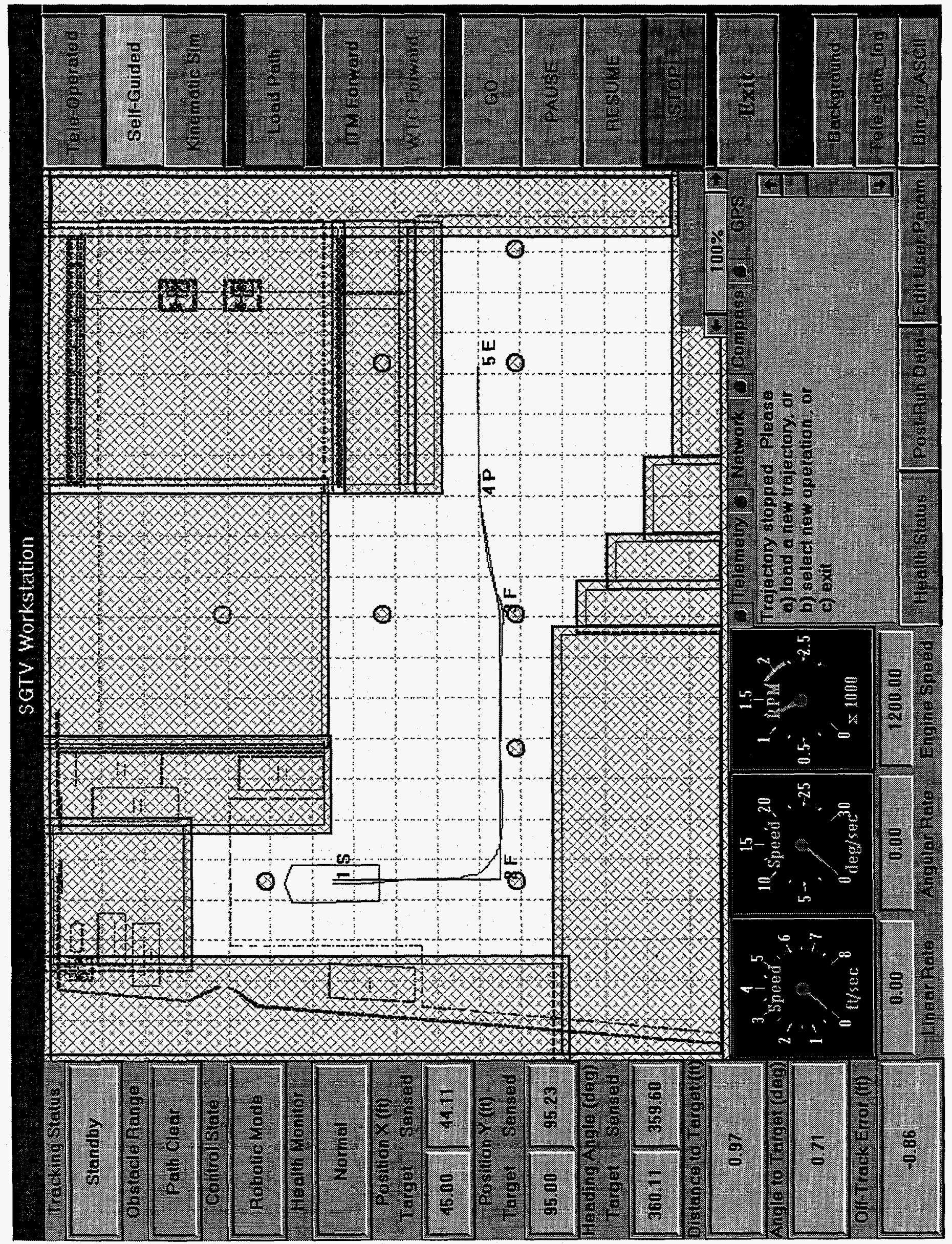




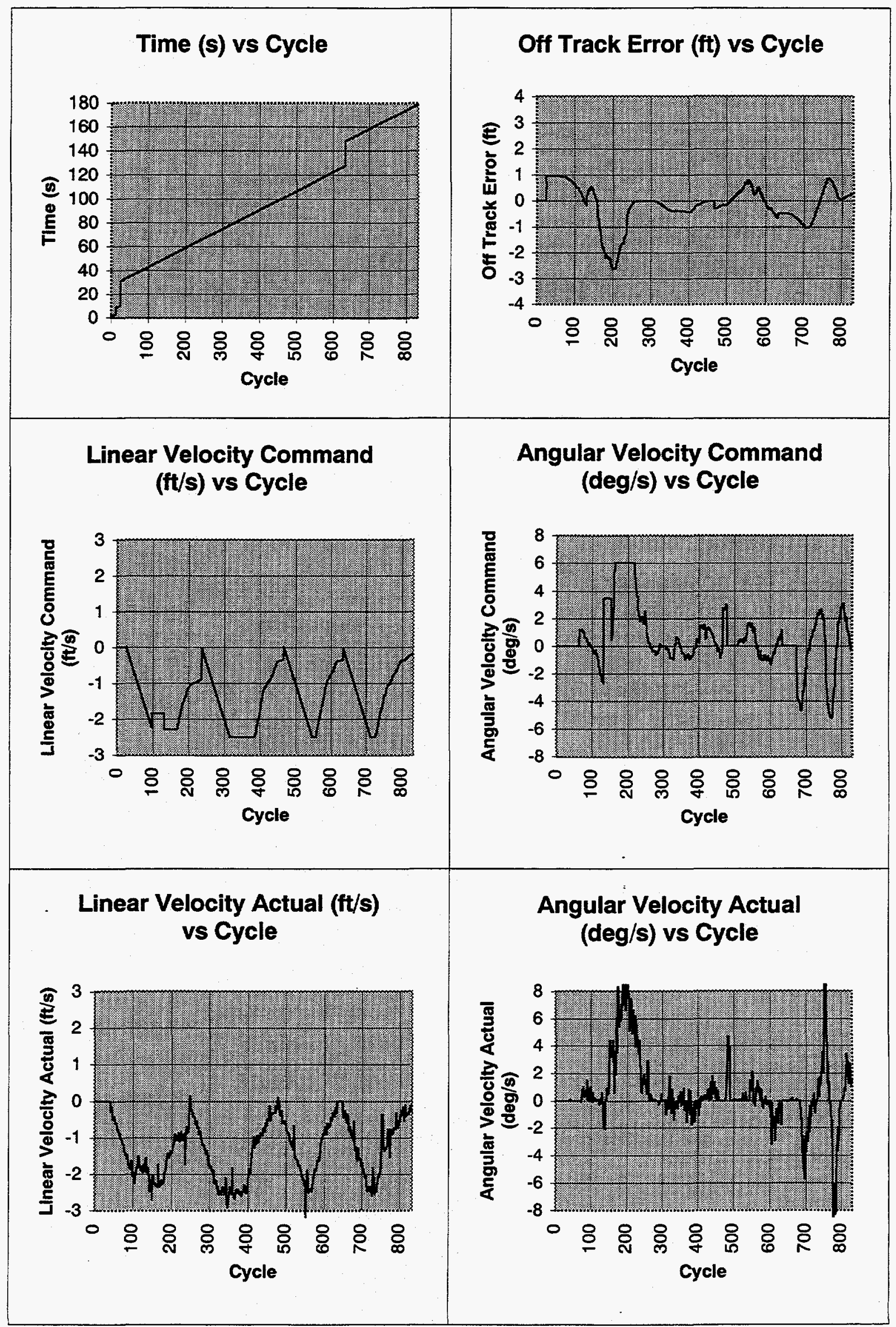

TP1_R10 


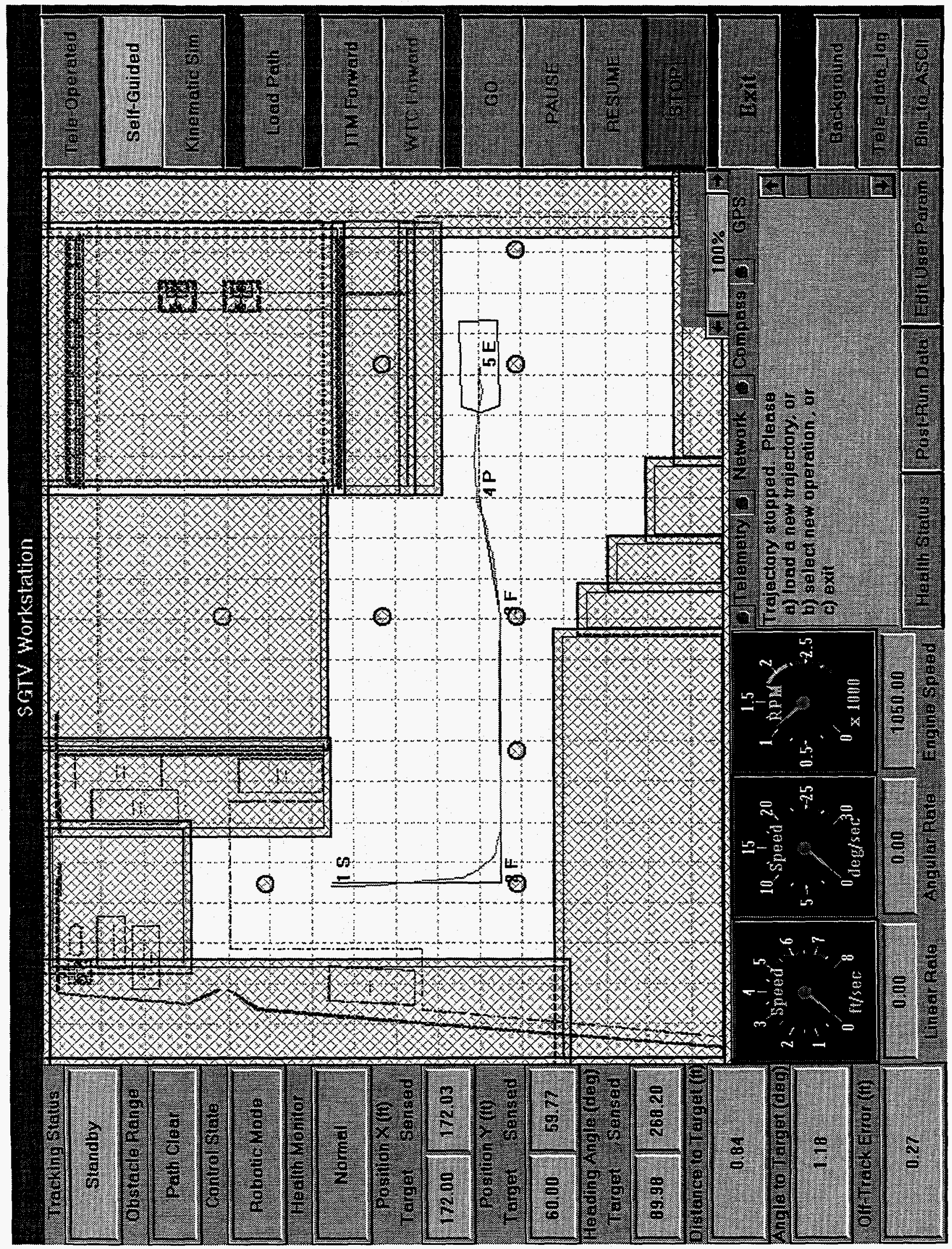




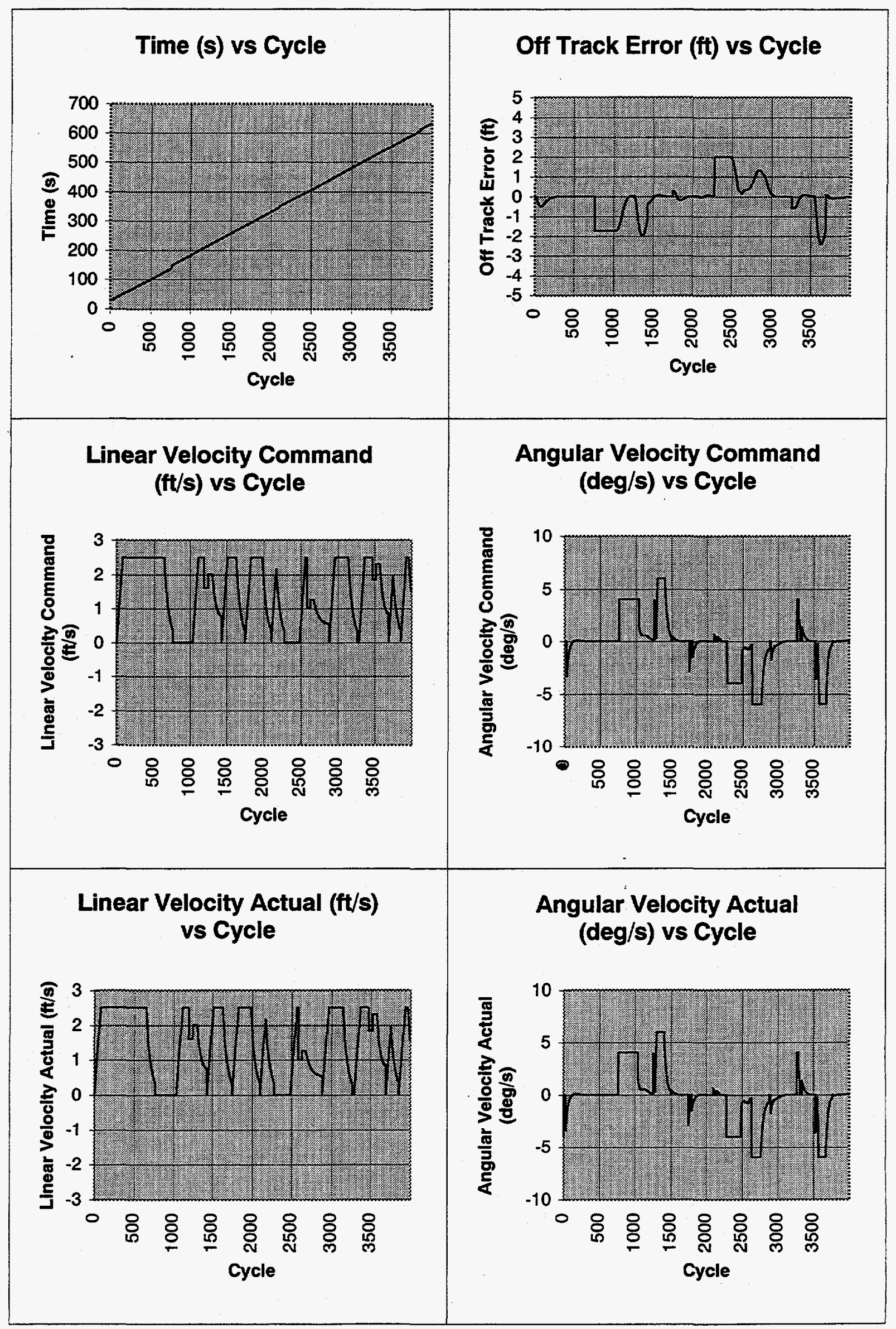




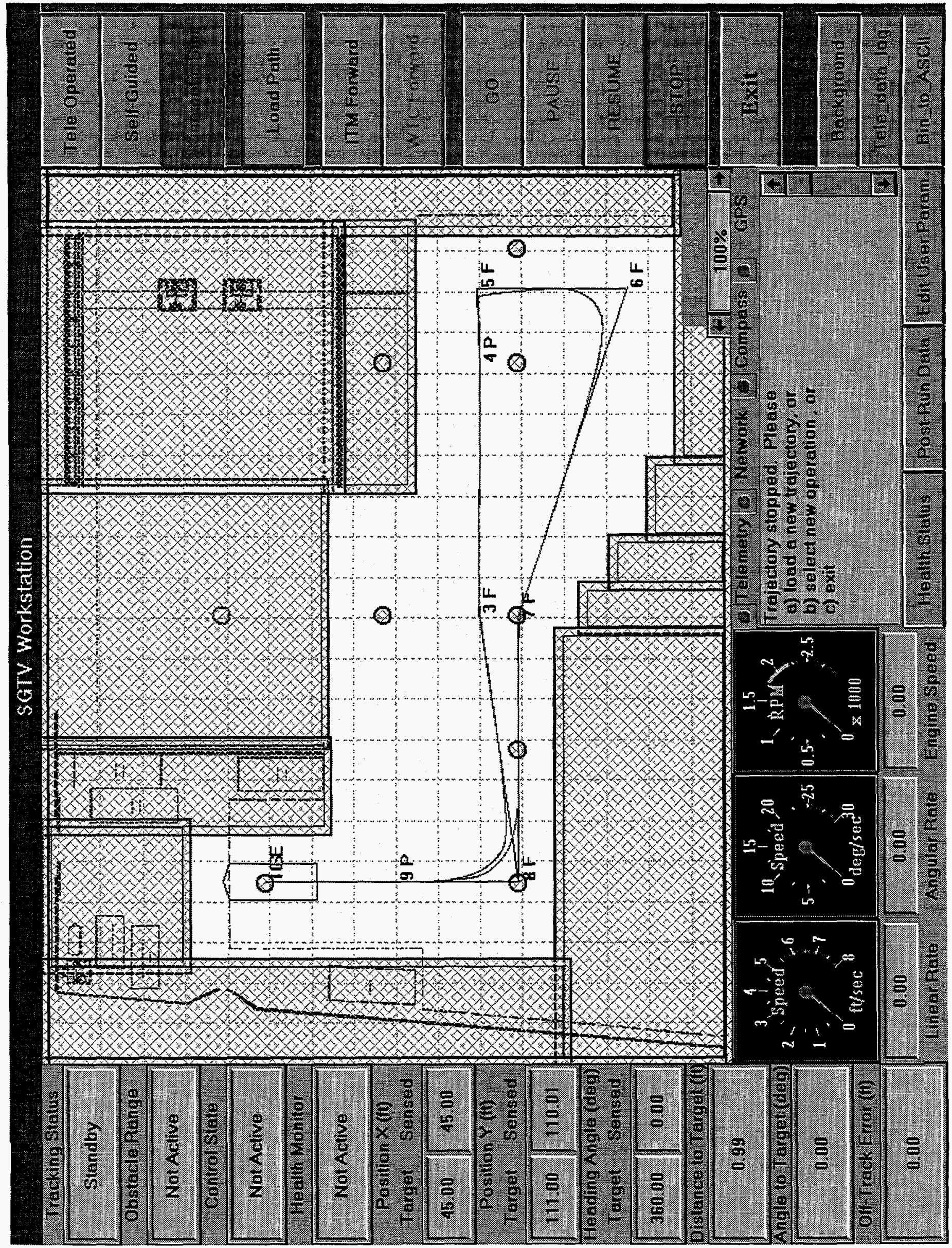




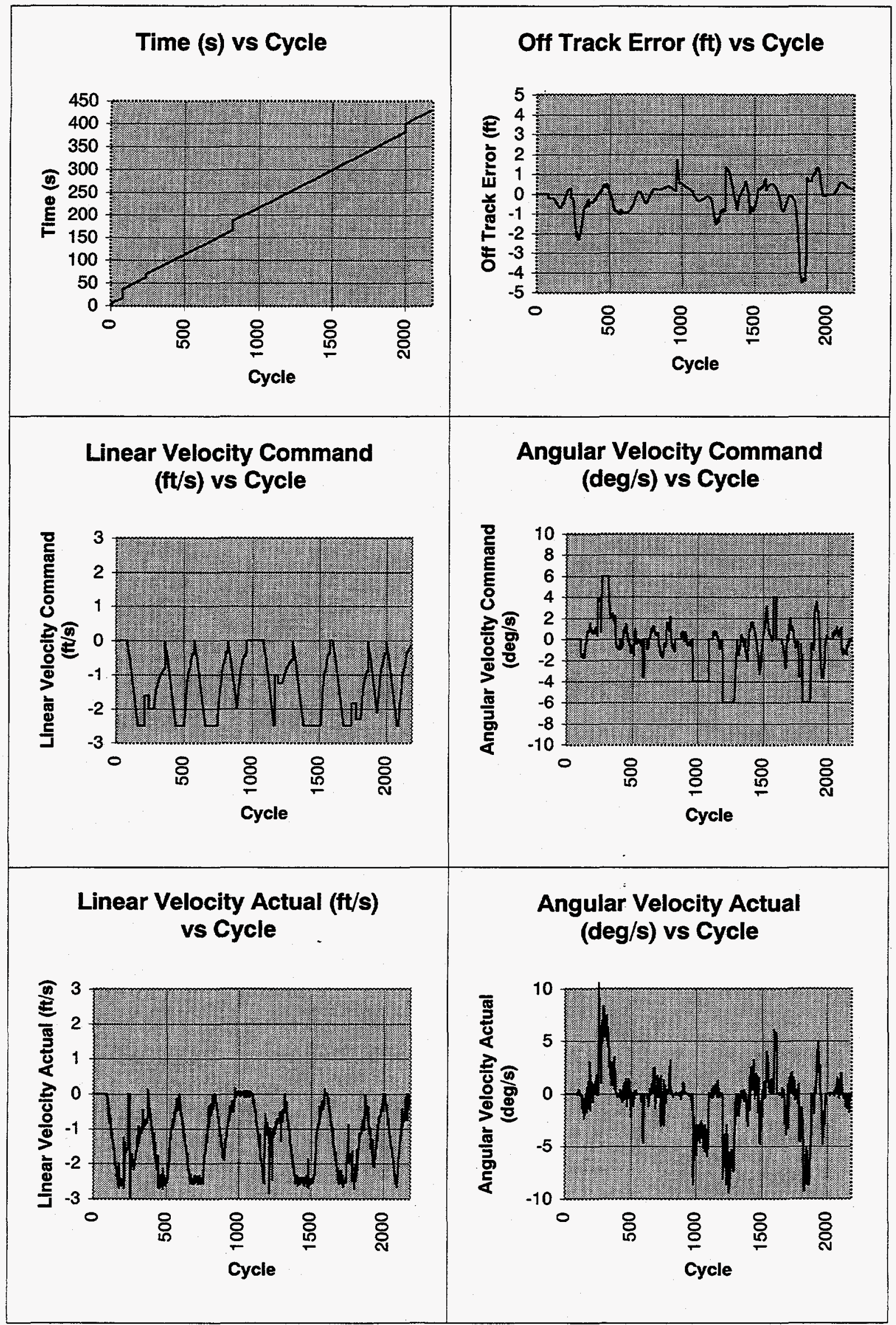


โป $2 \mathrm{dL}$

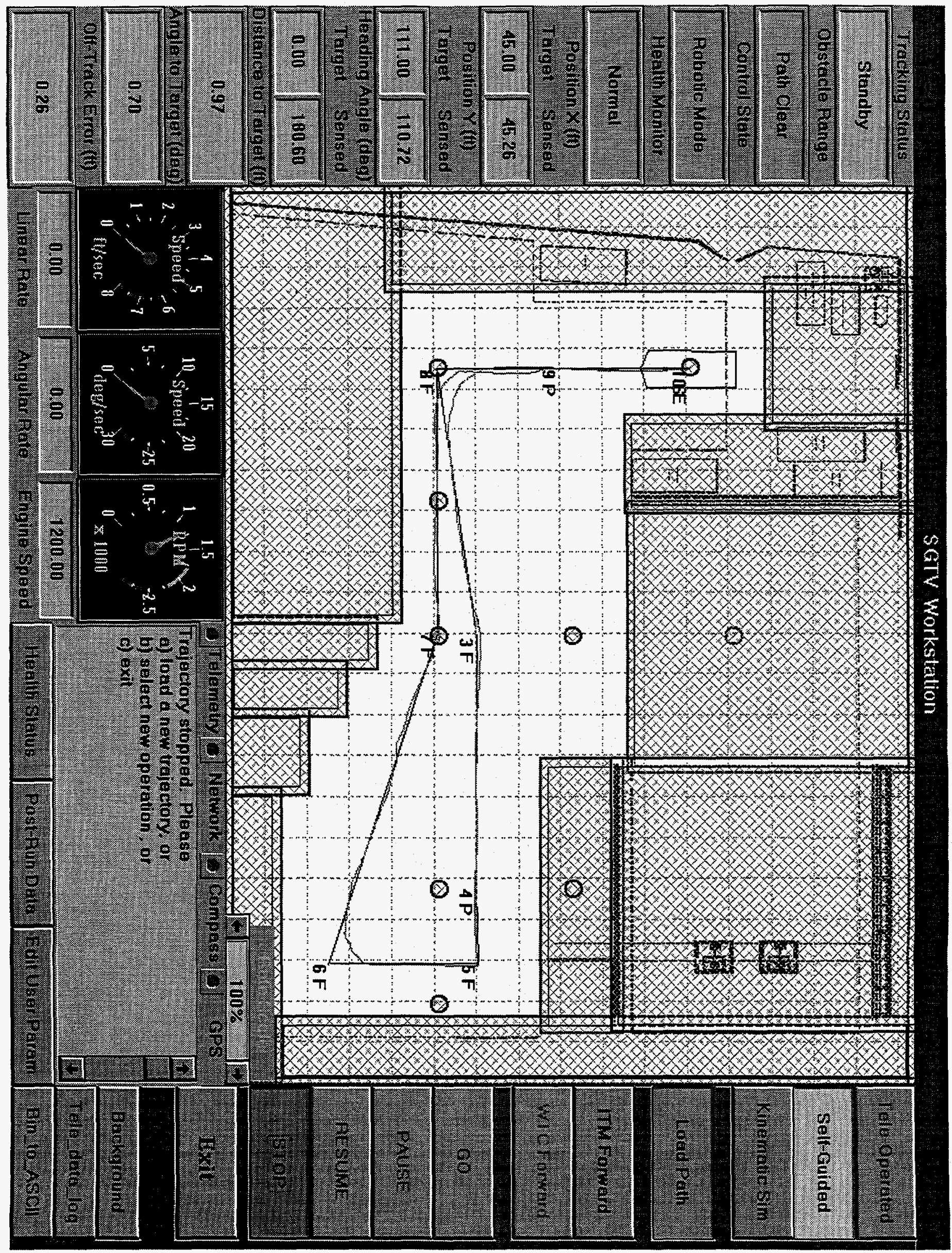




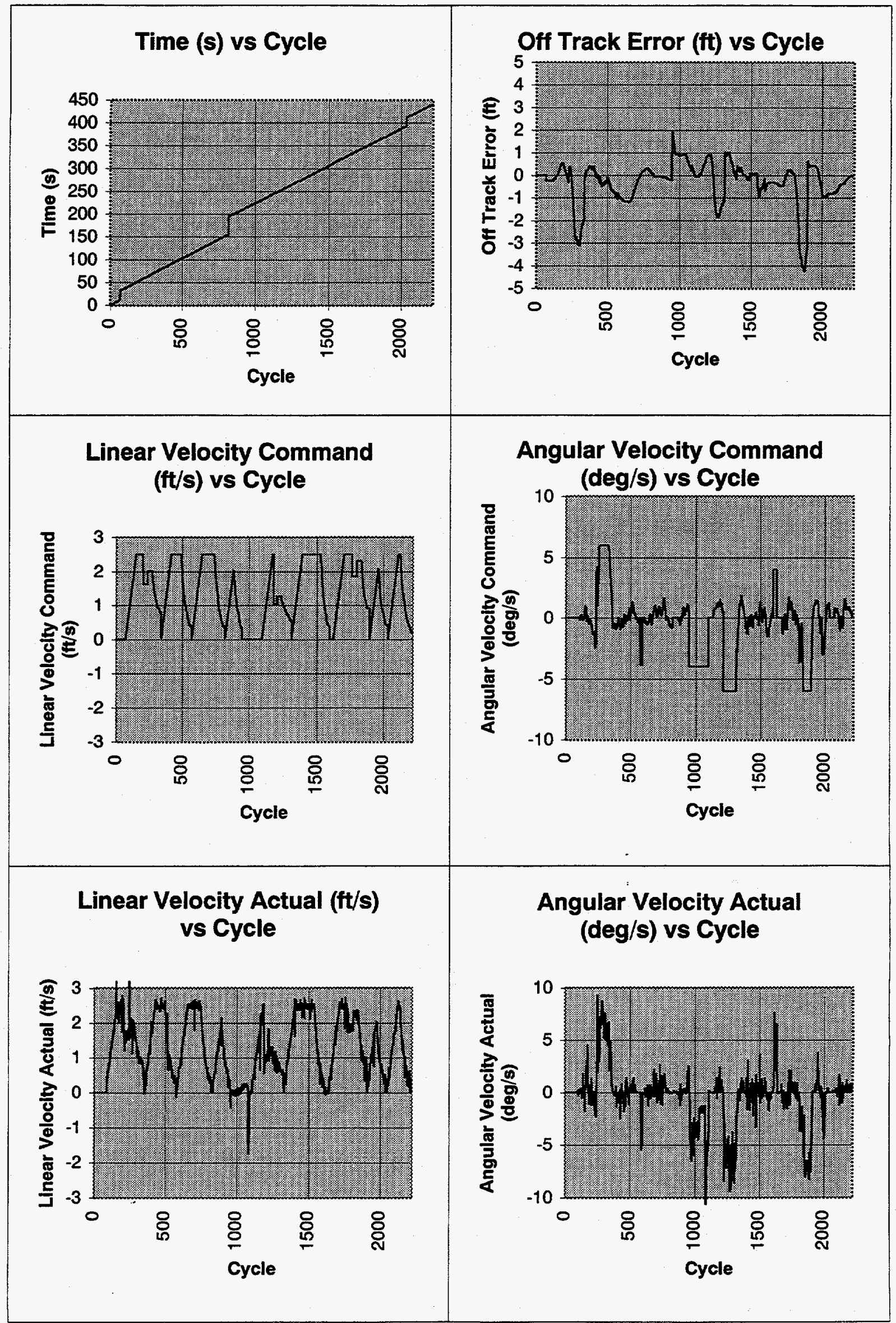

TP2_R2 


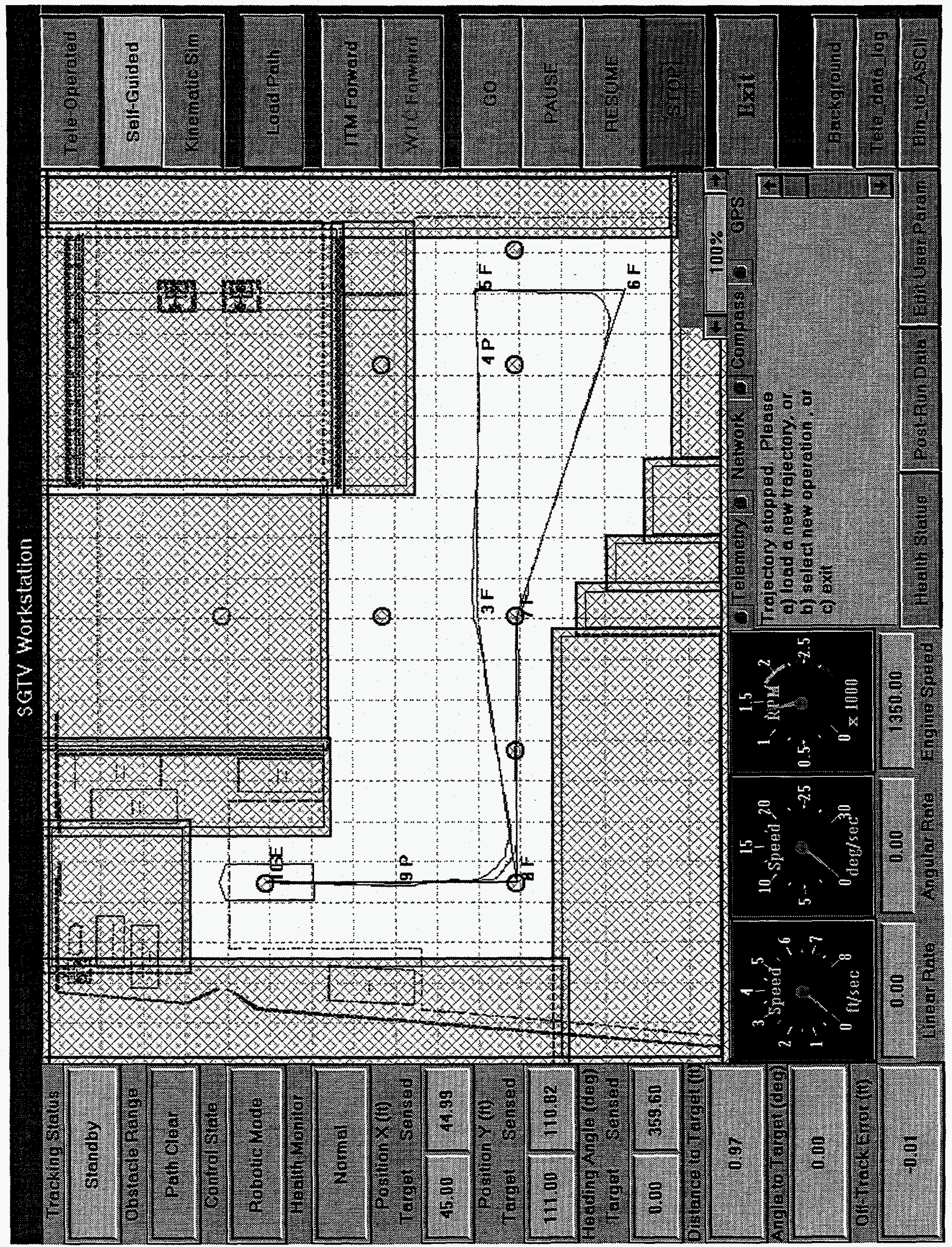




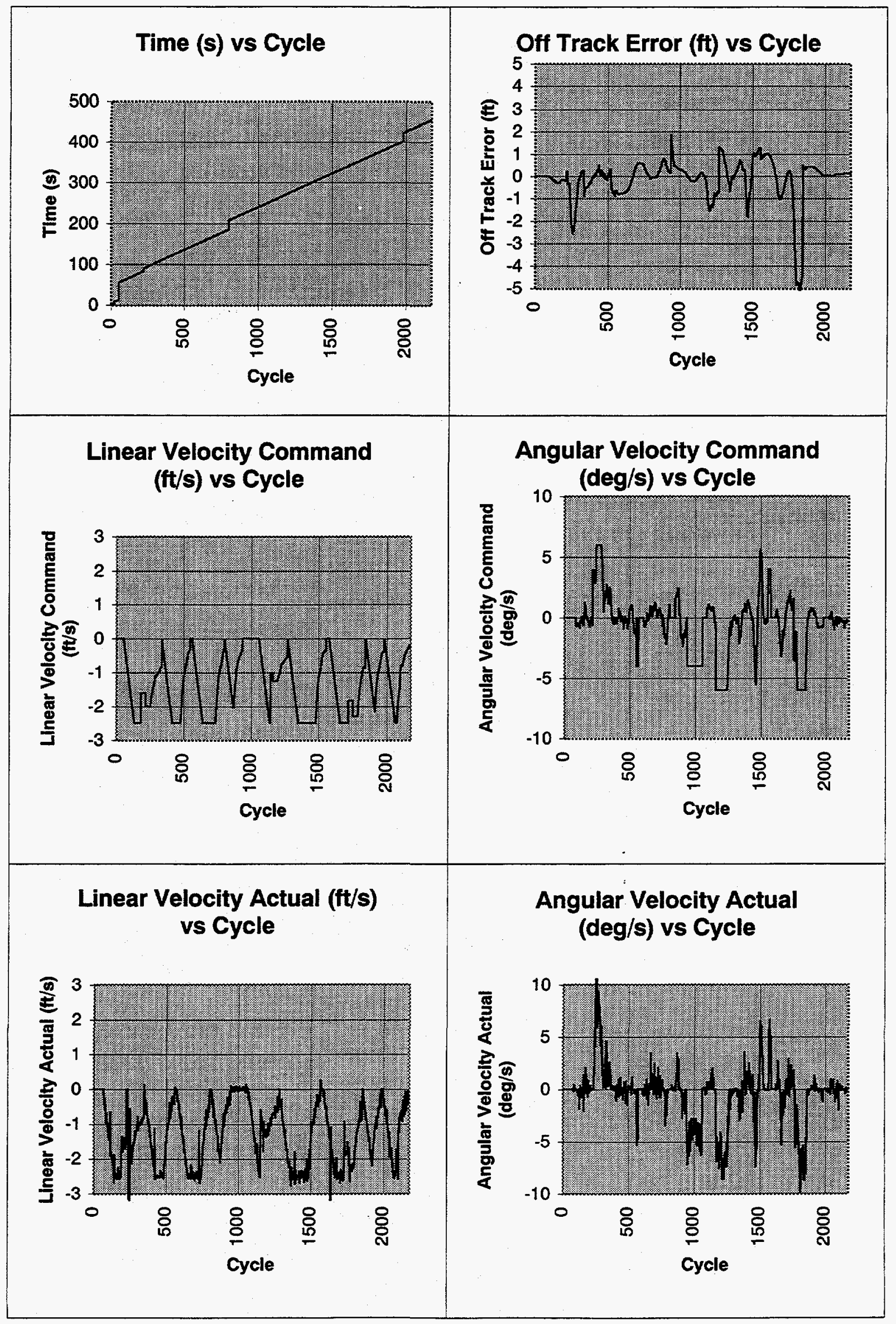


$\varepsilon ل^{-} \mathrm{dd}$

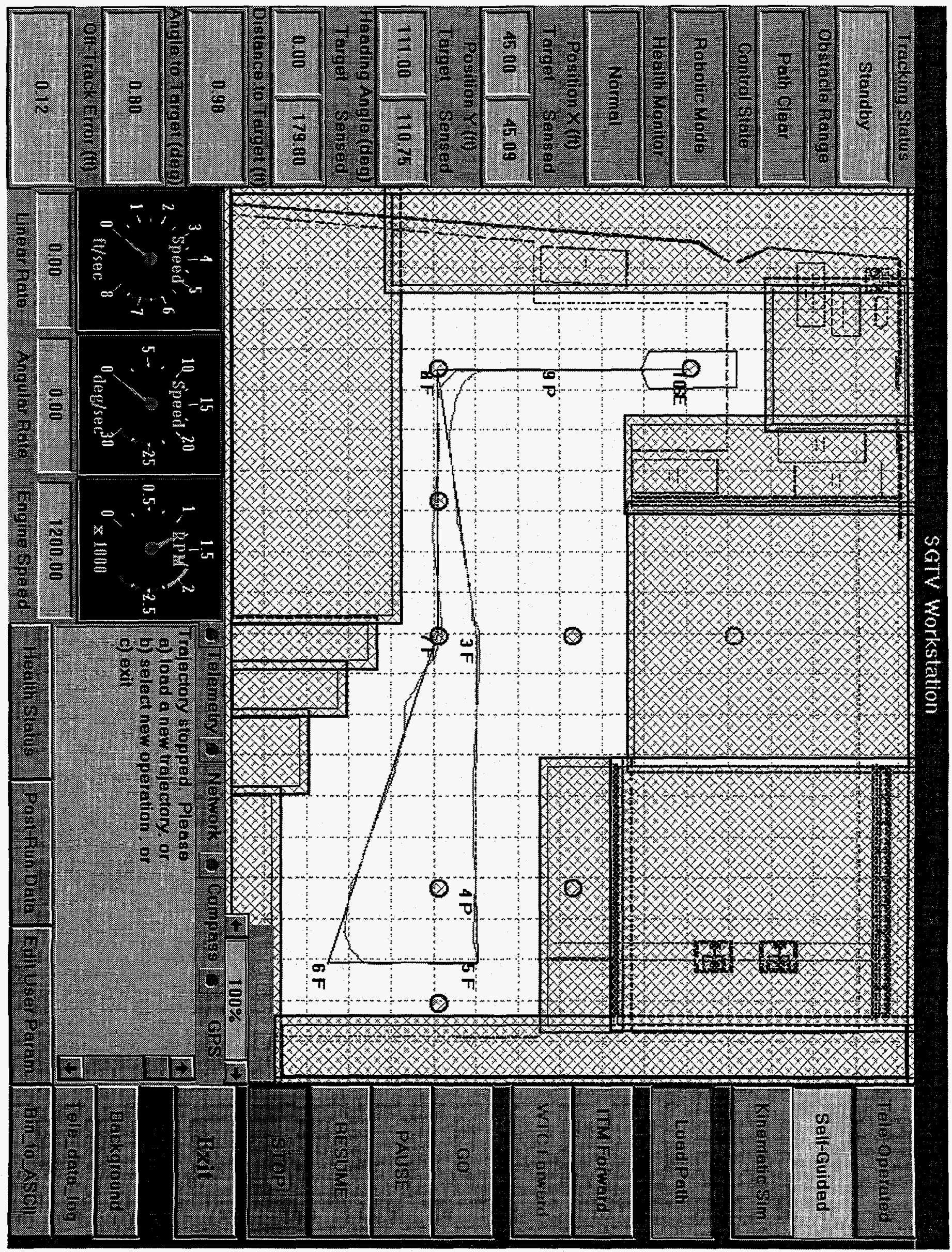




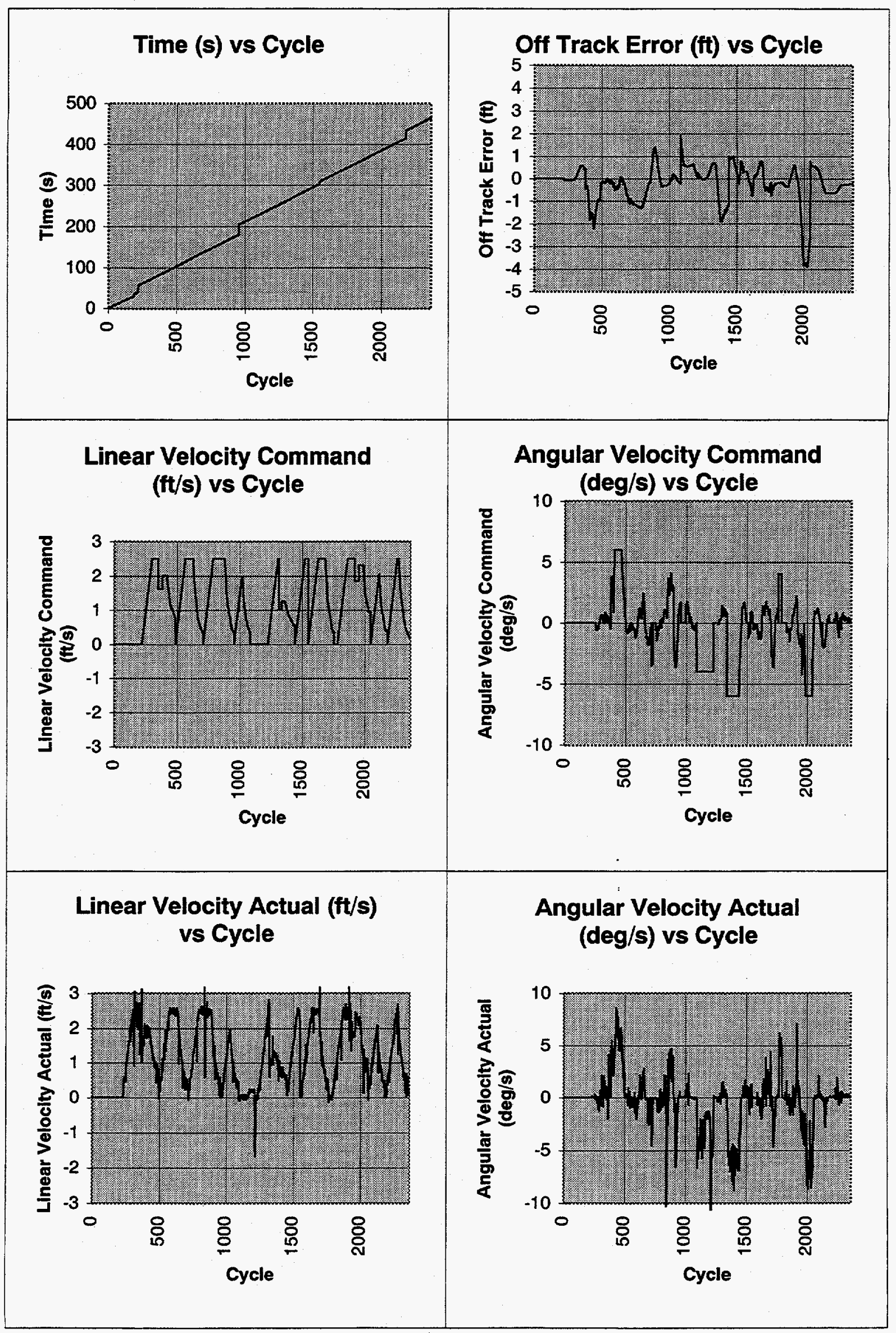




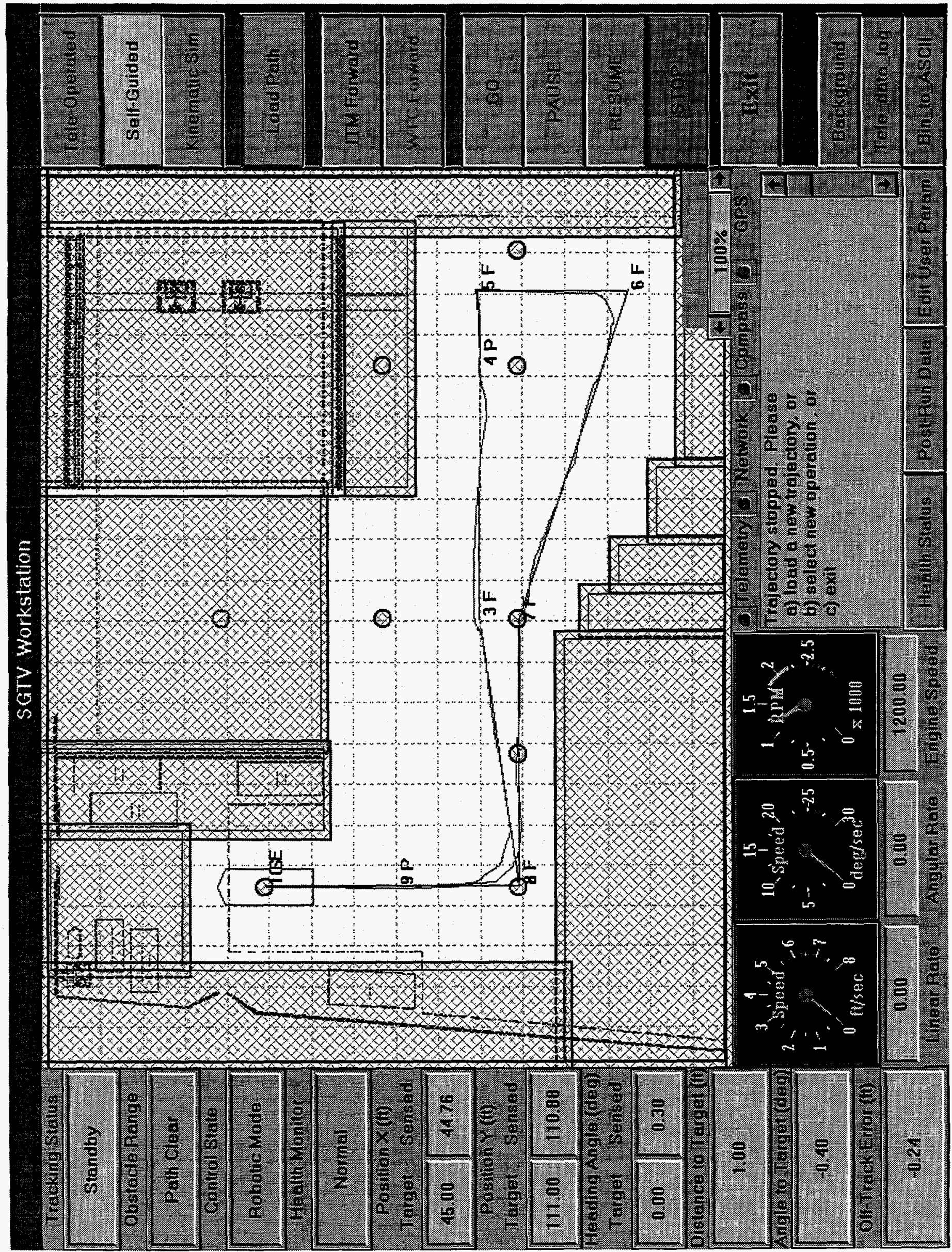




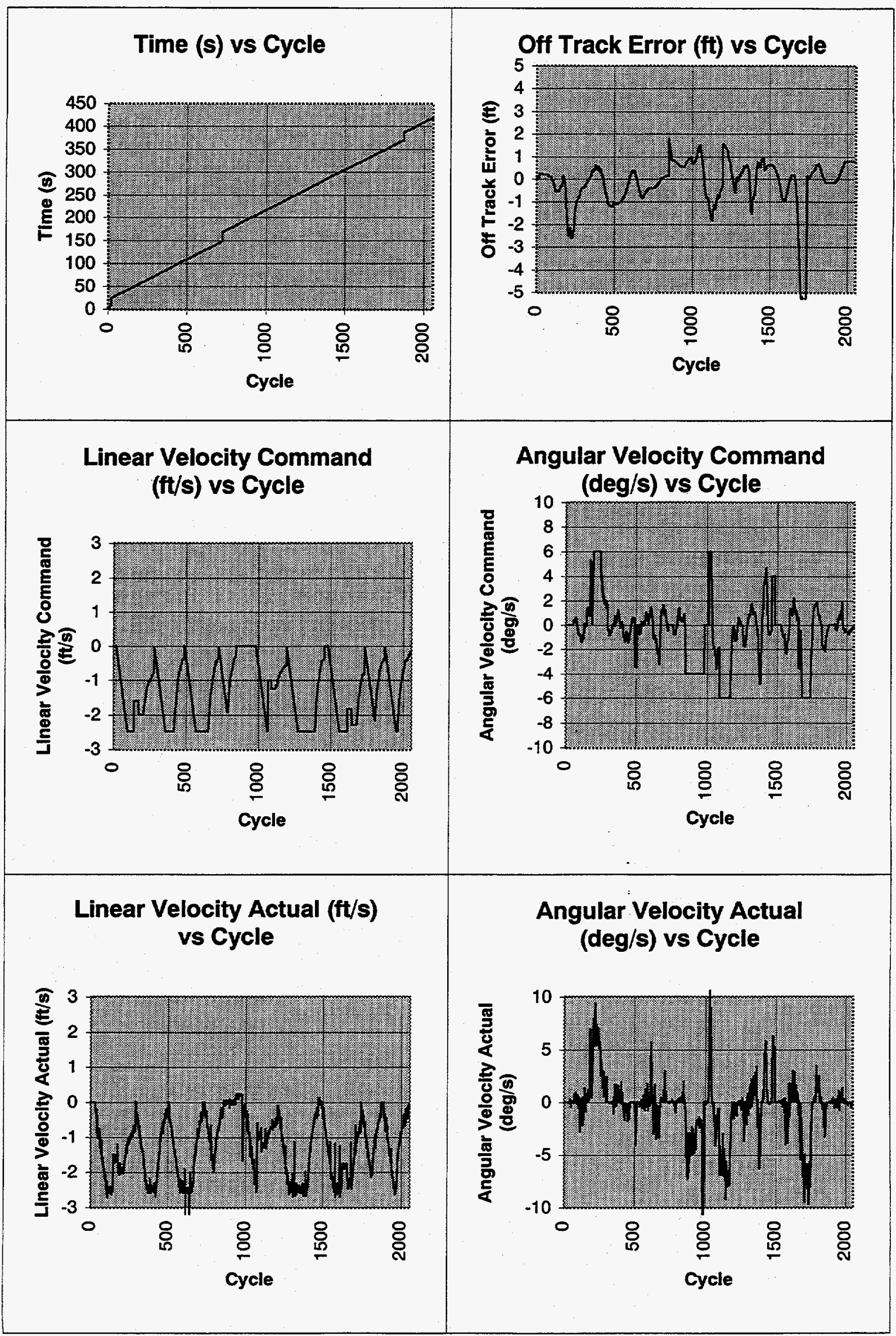




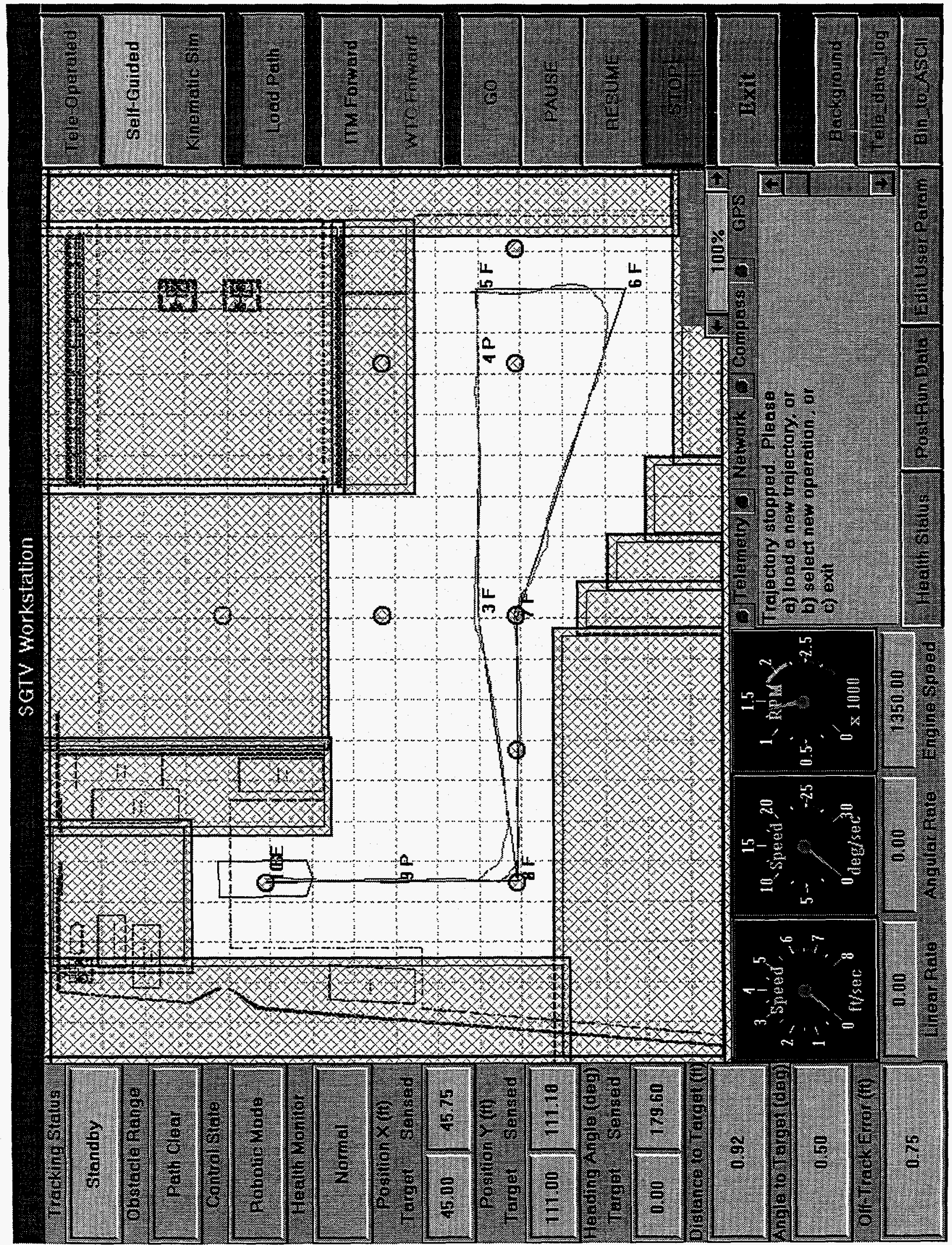




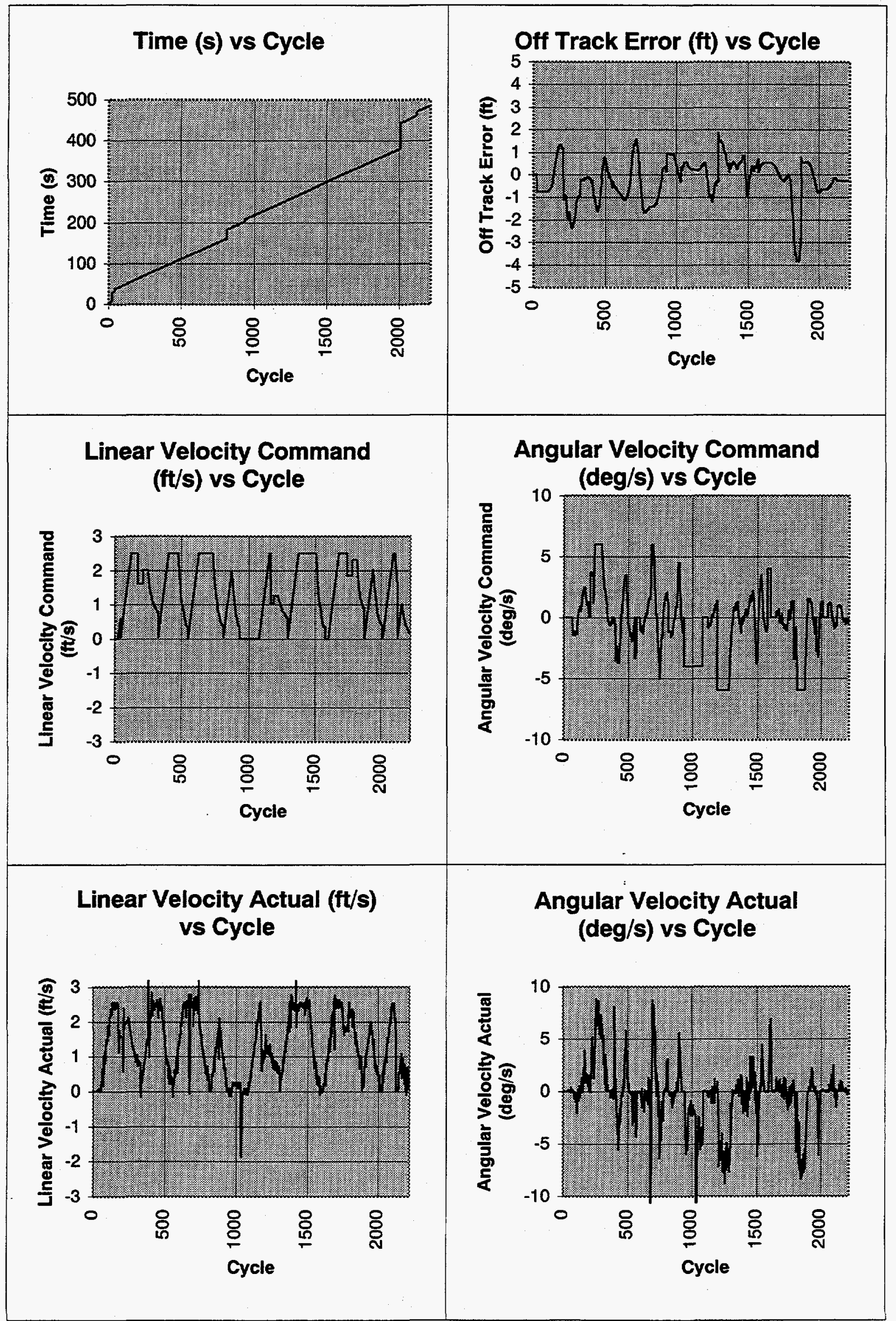

TP2_R6 


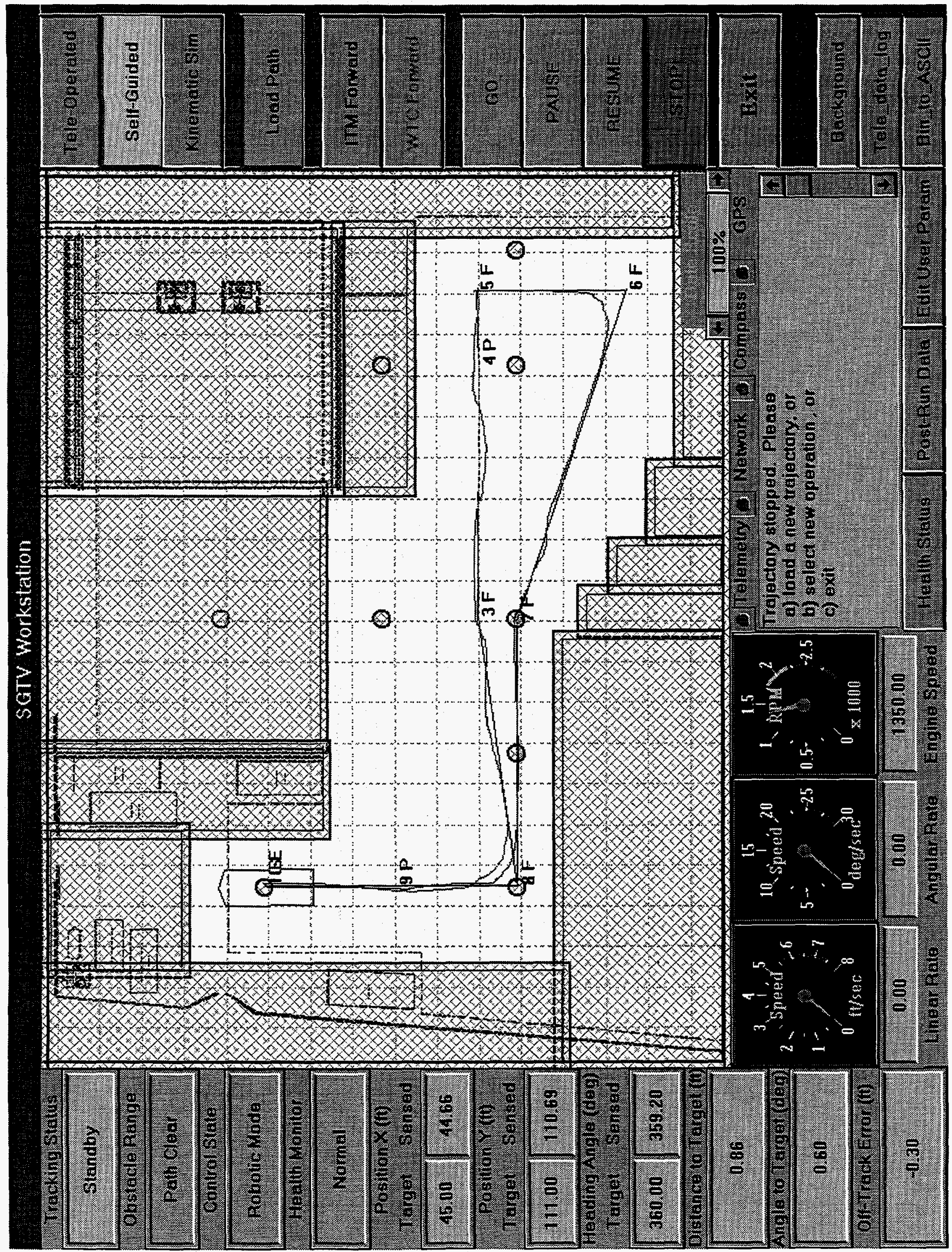




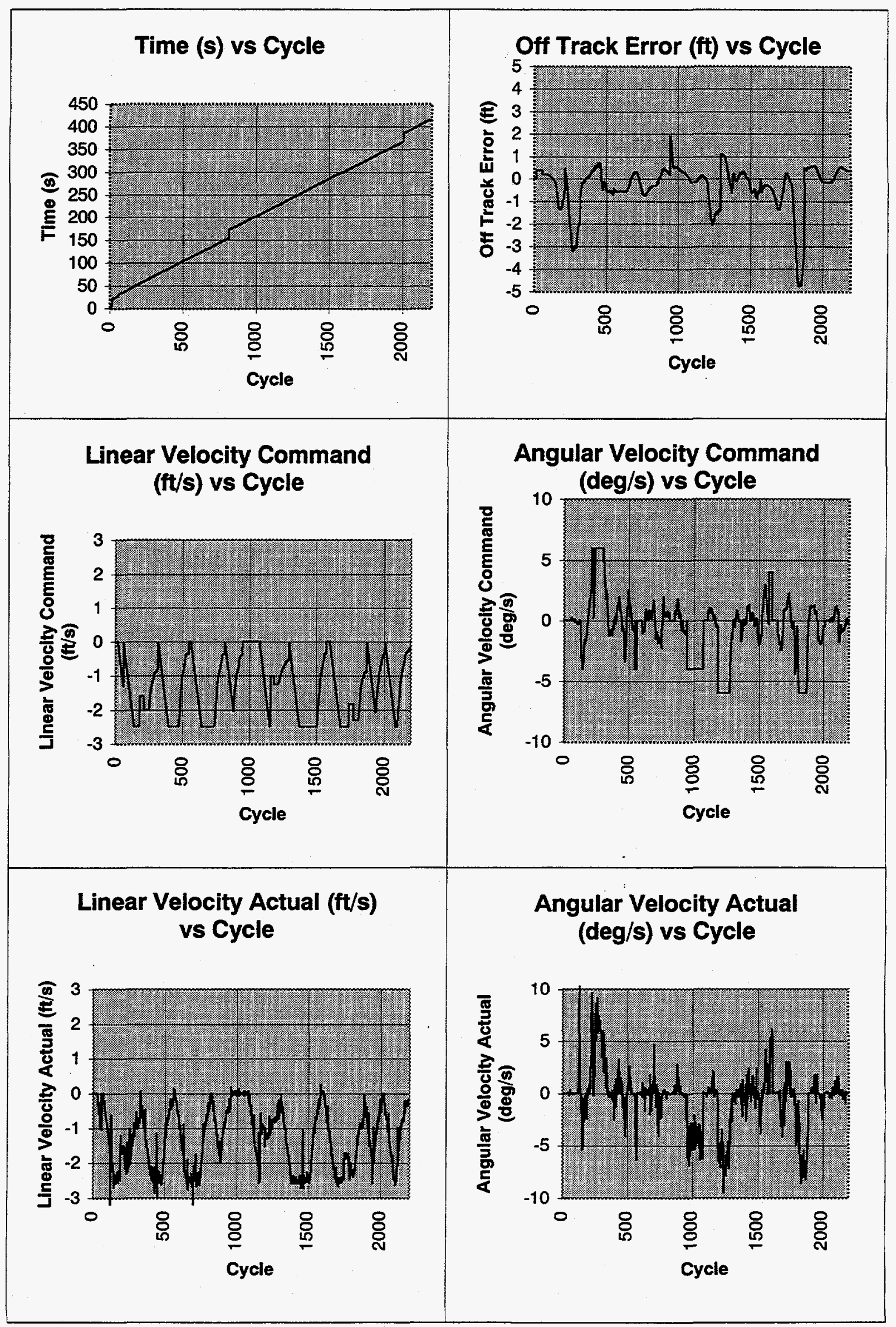




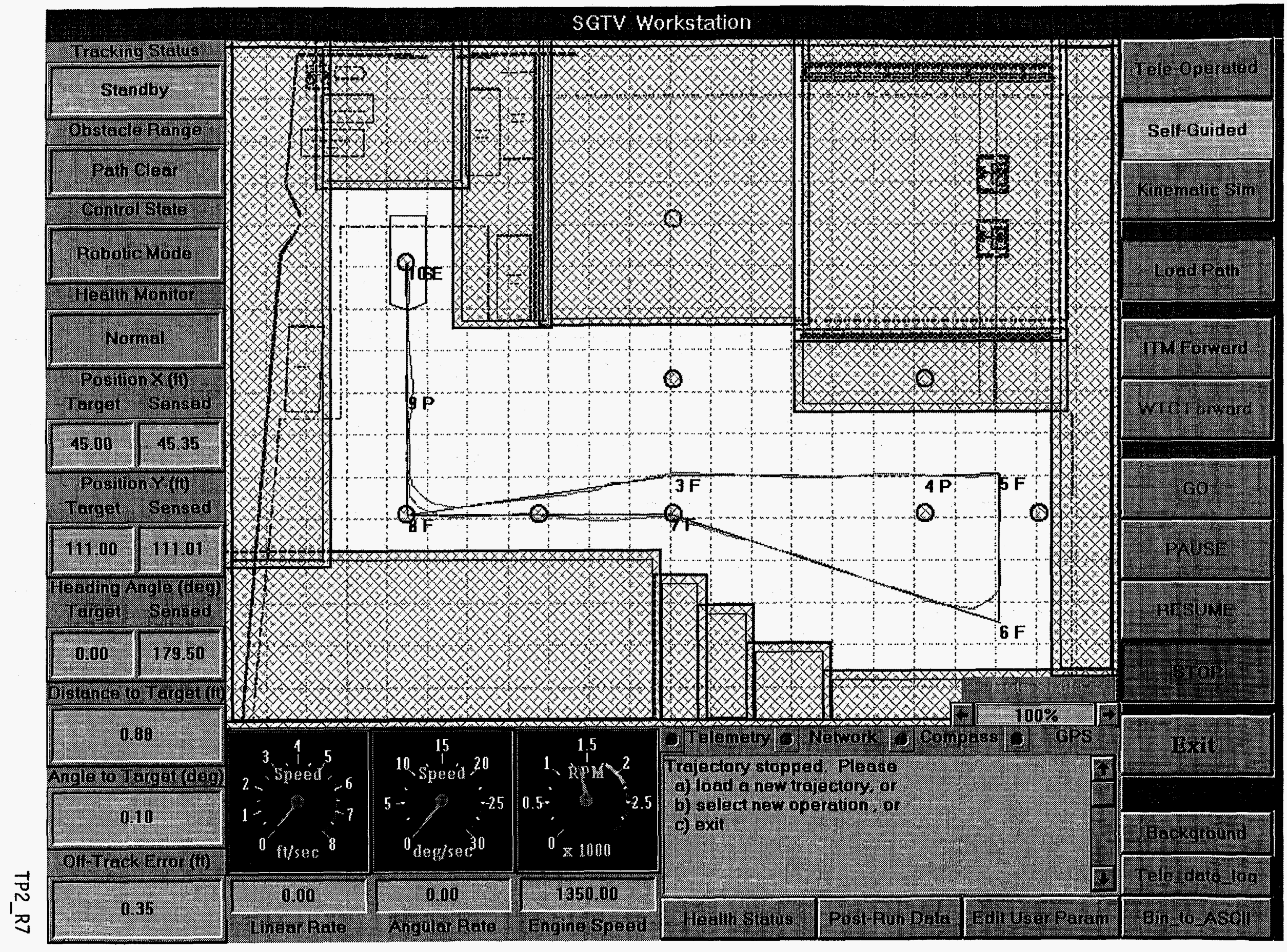




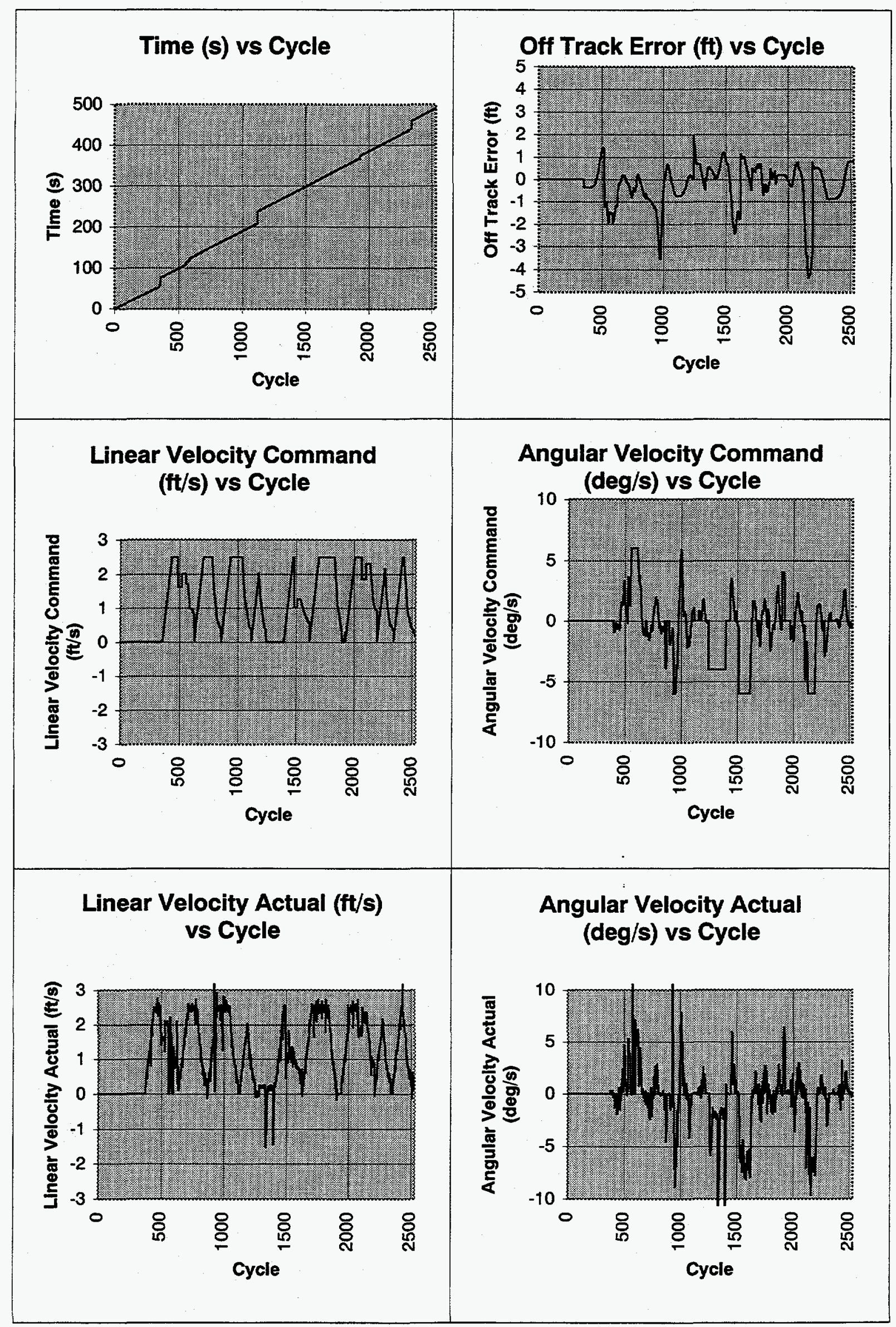

TP2_R8 


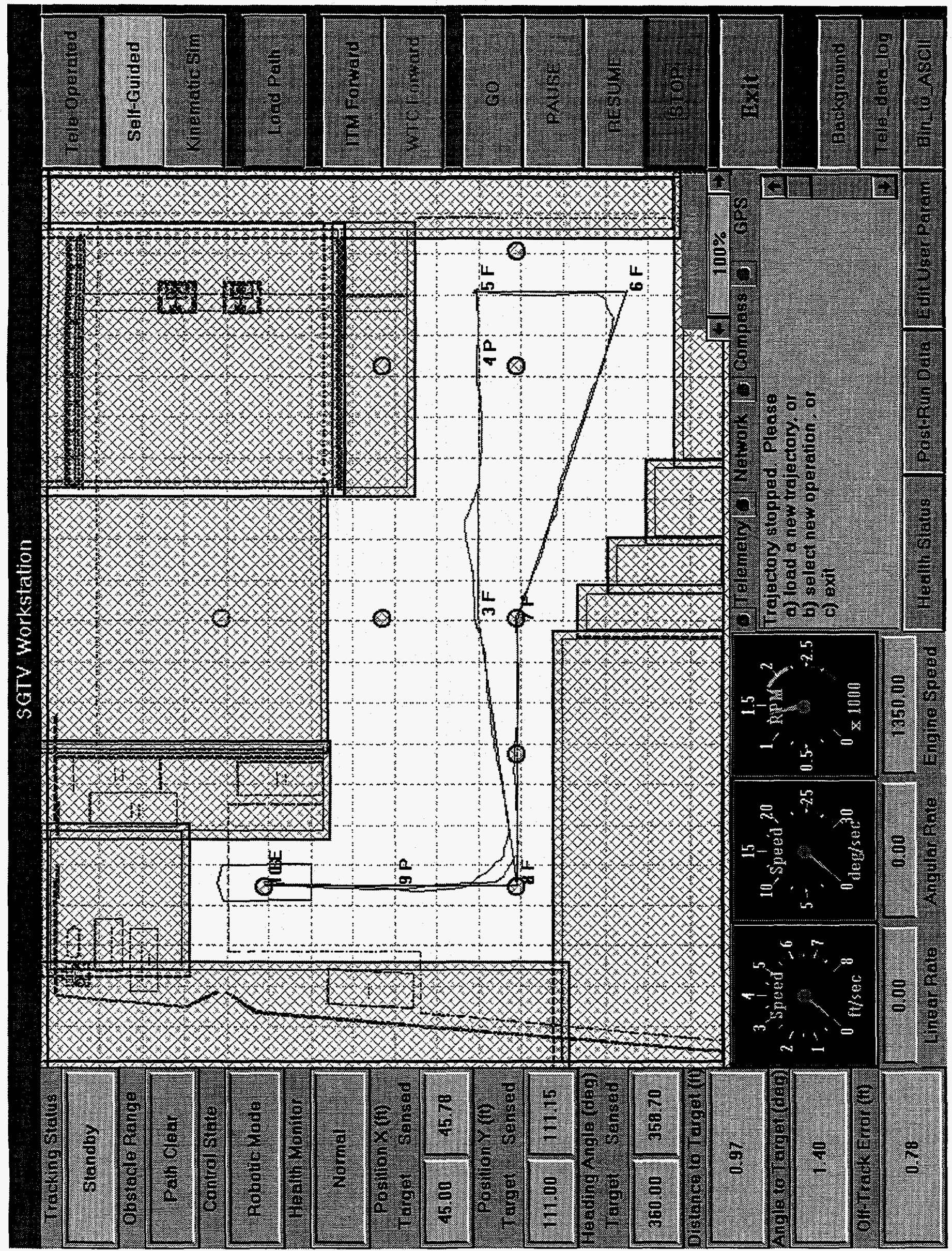




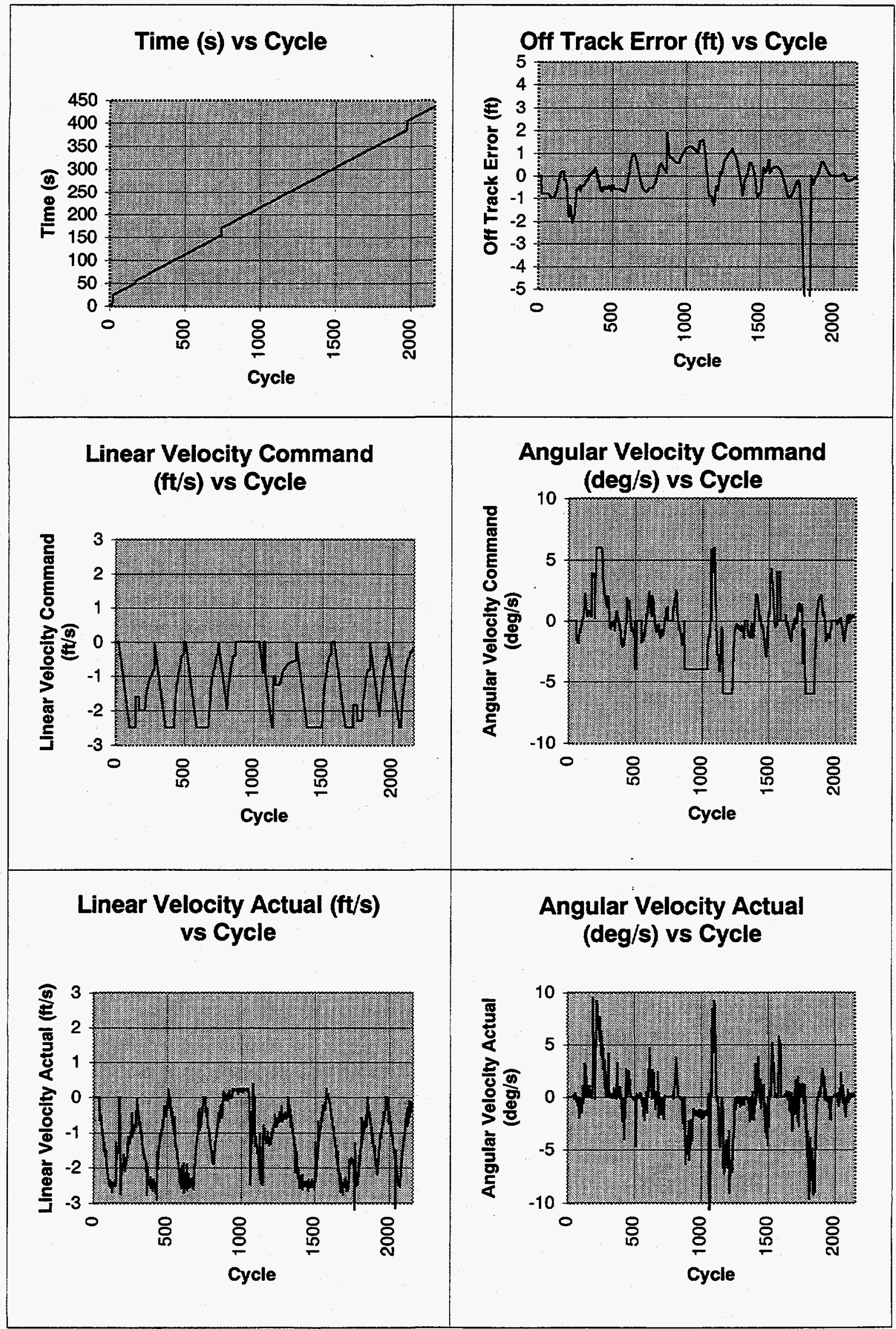


$6 \mathrm{y}^{-} \mathrm{Zd1}$

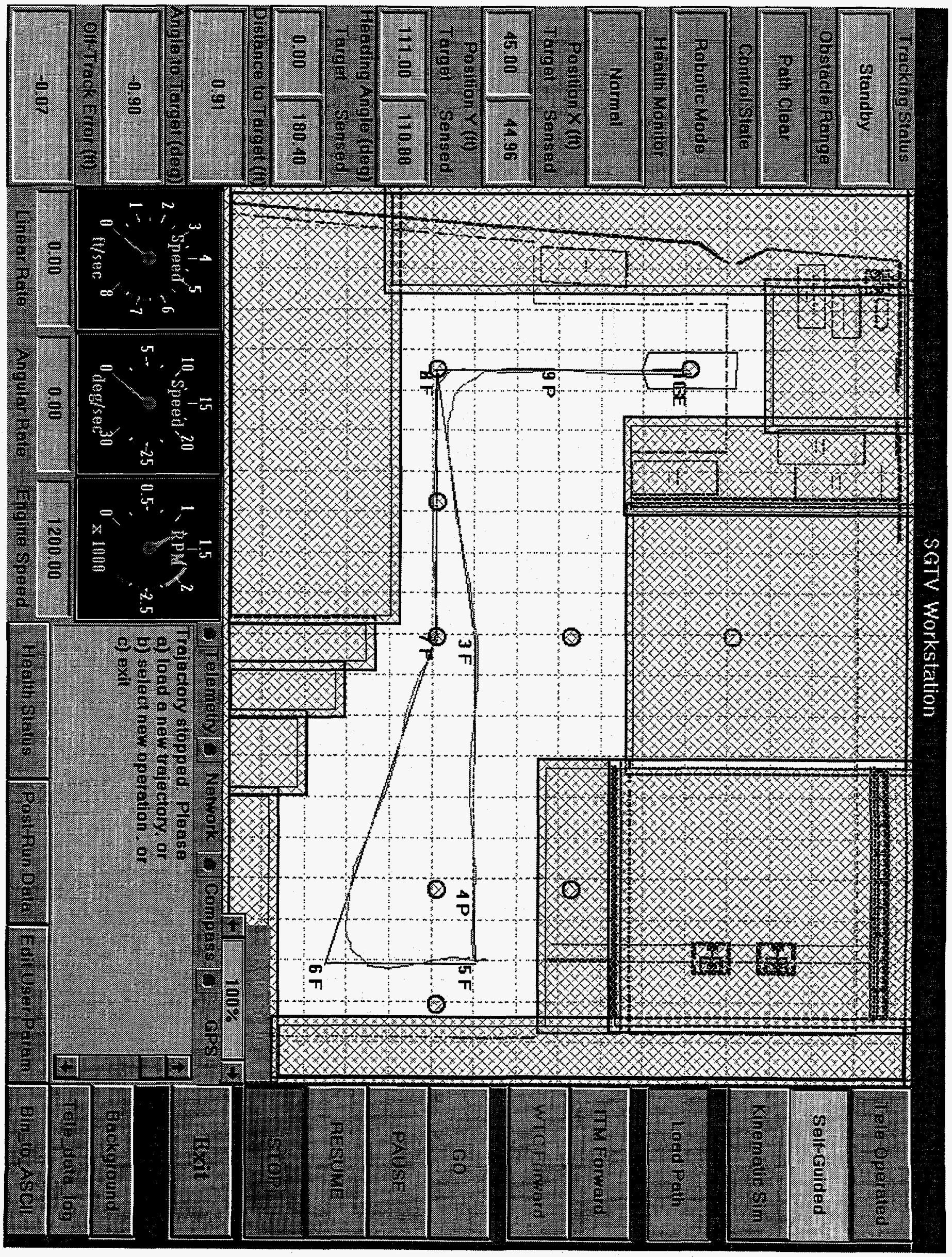




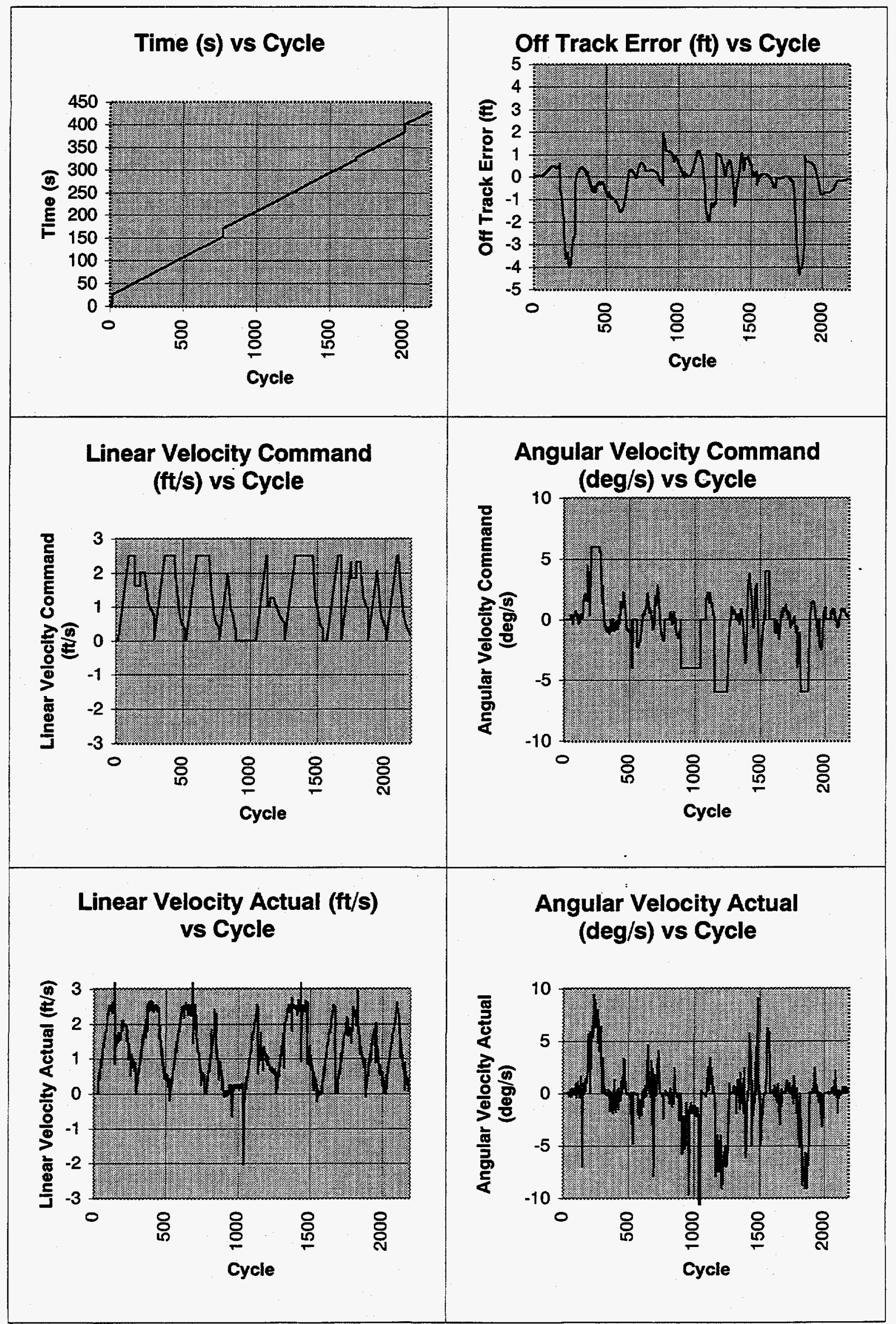




\section{0โร $2 \mathrm{dI}$}

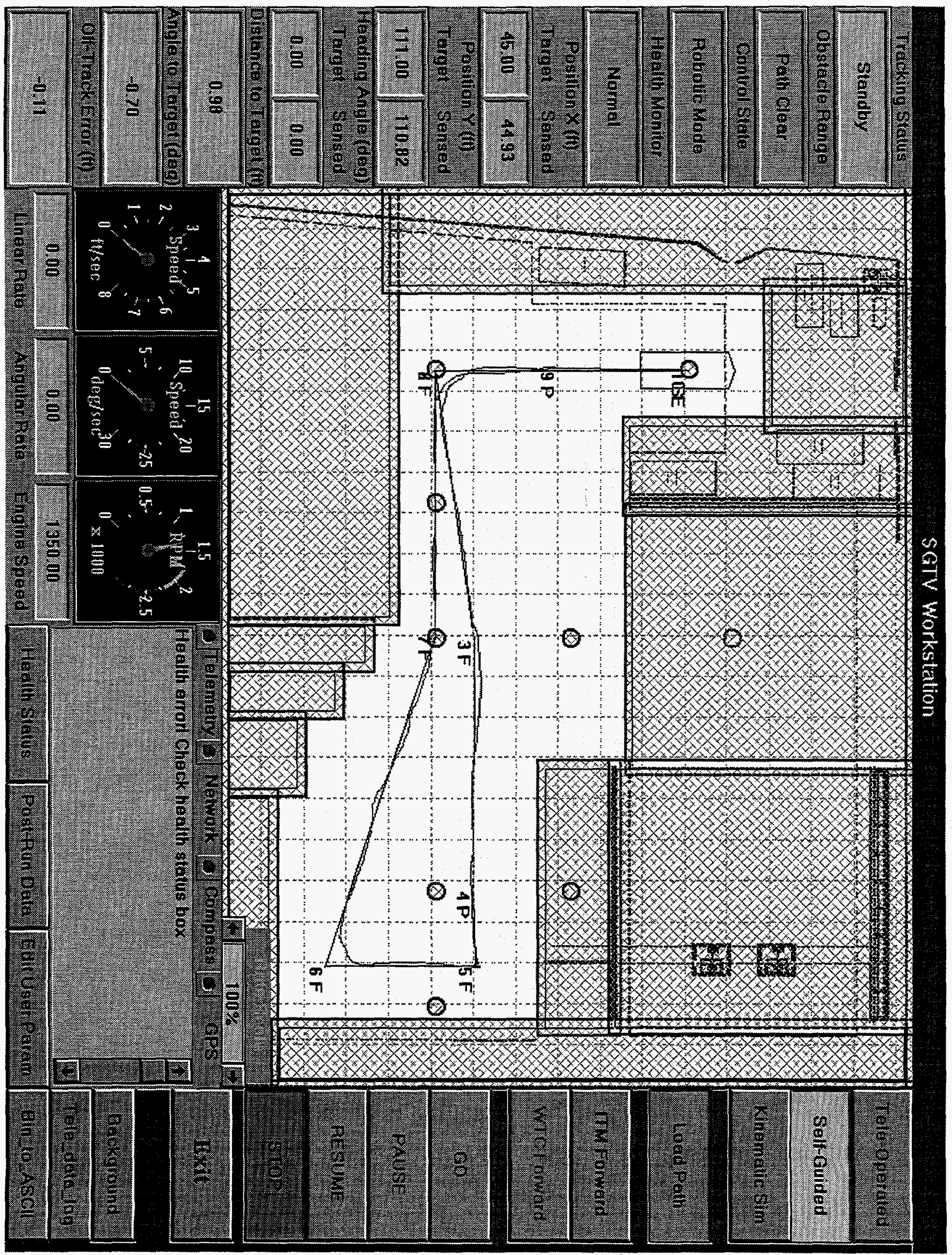




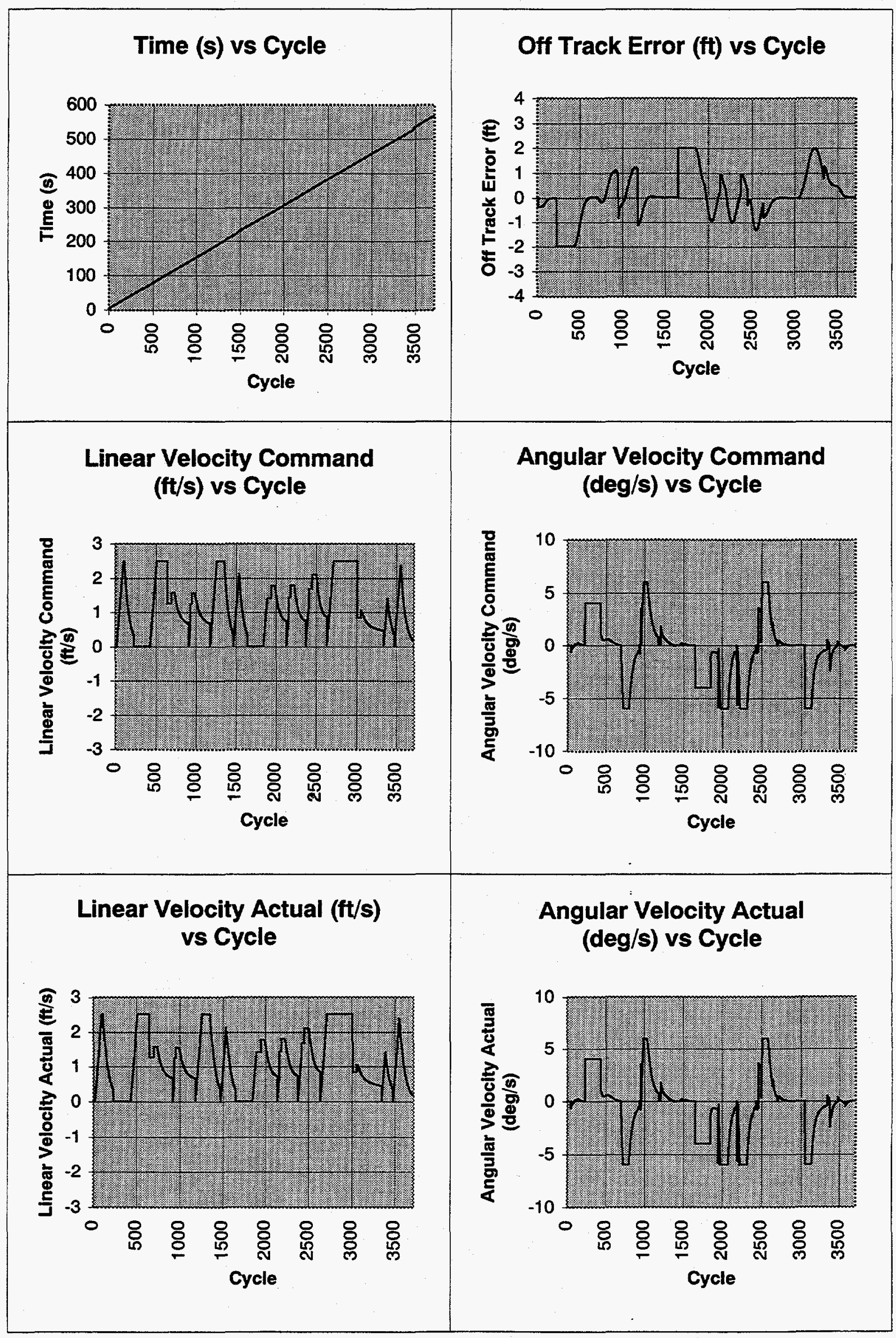




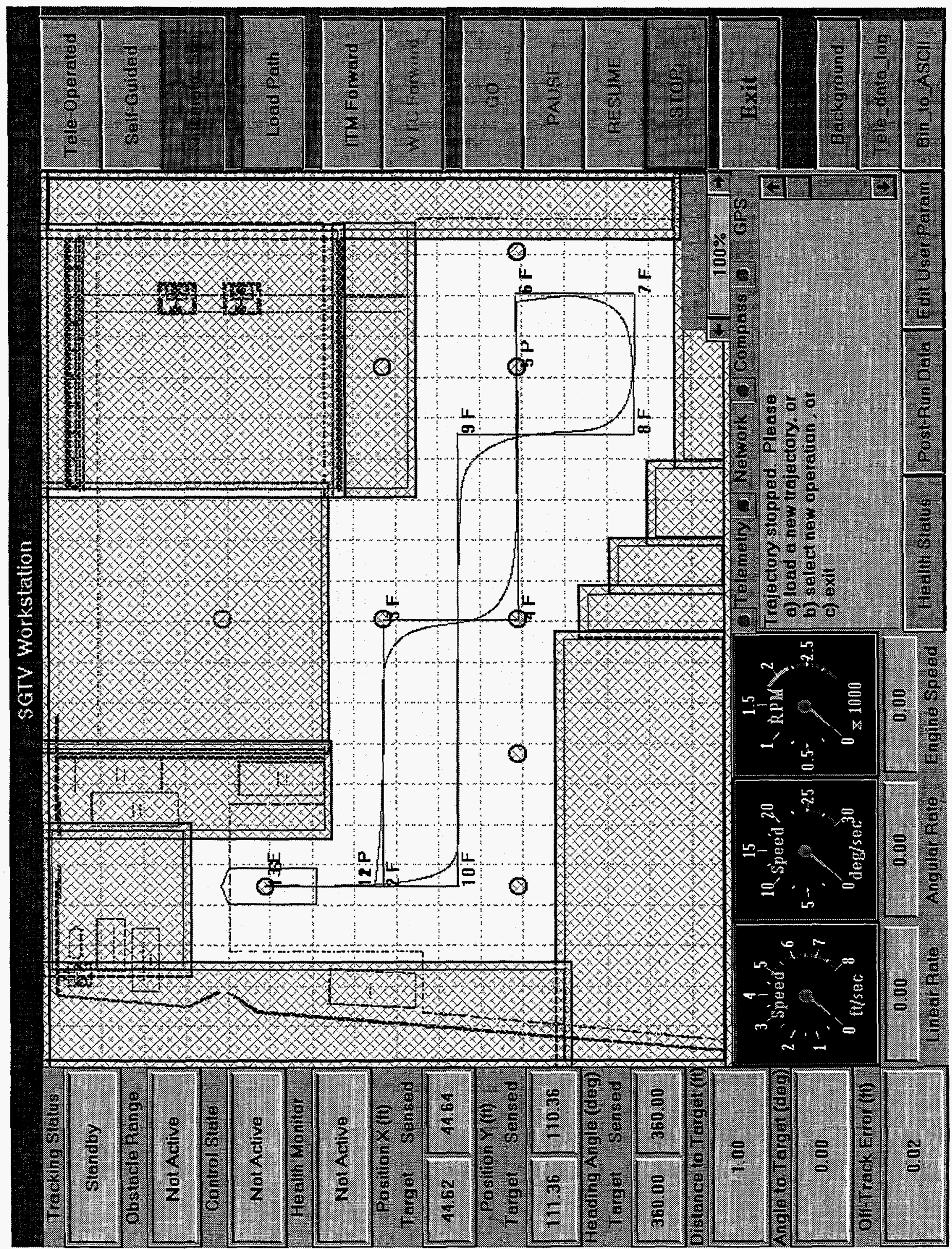




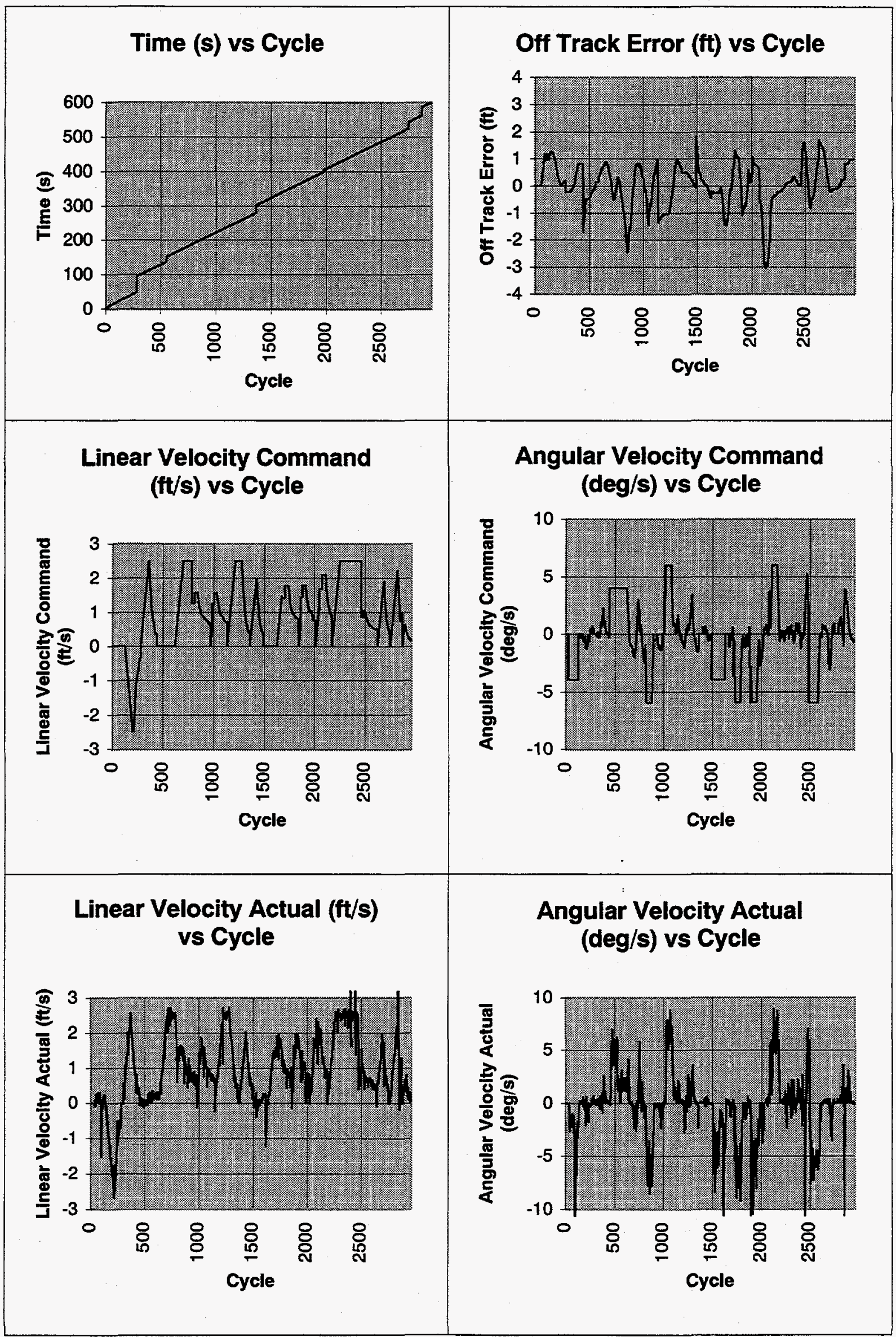


I ${ }^{-} \varepsilon d 1$

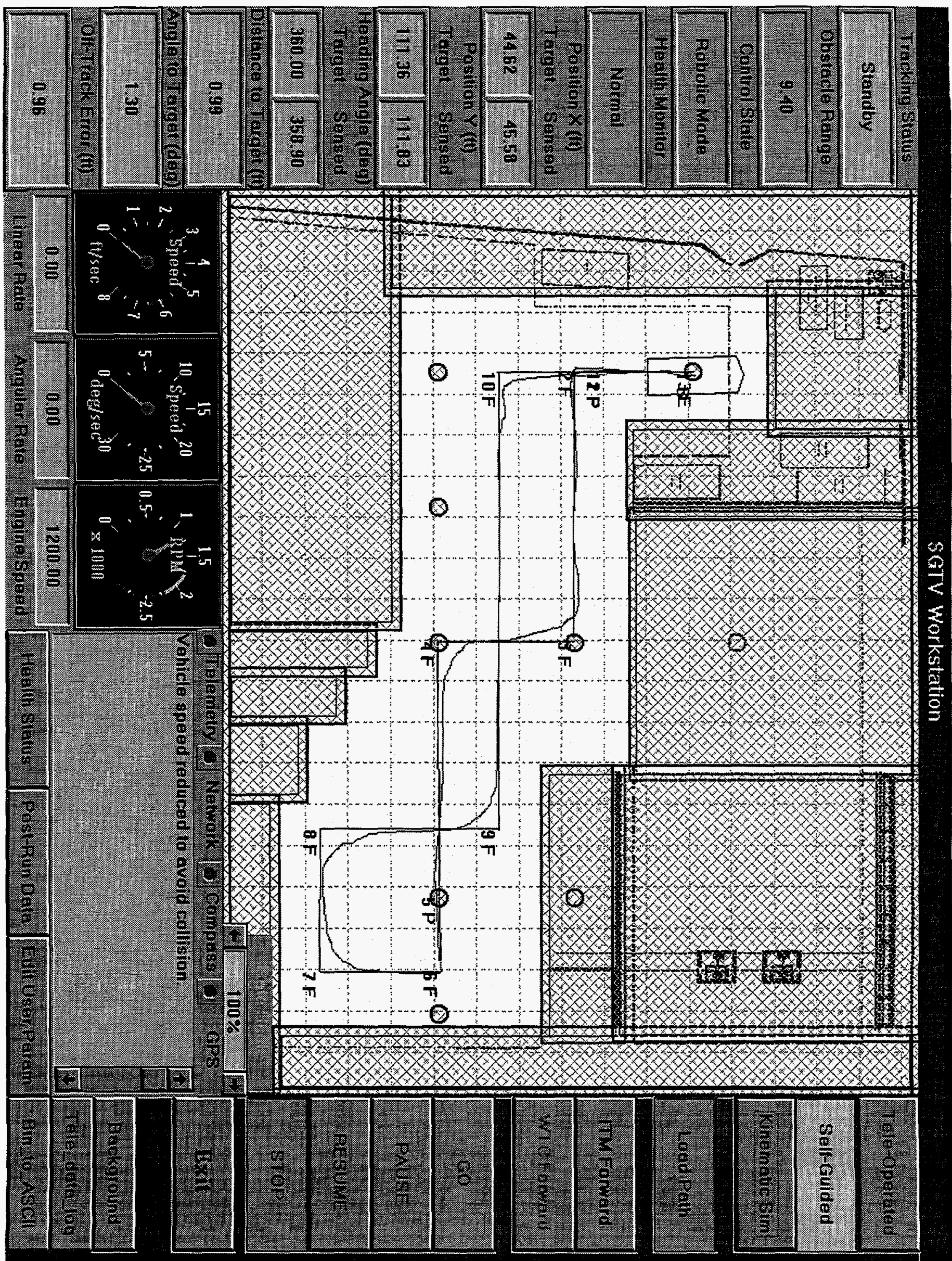




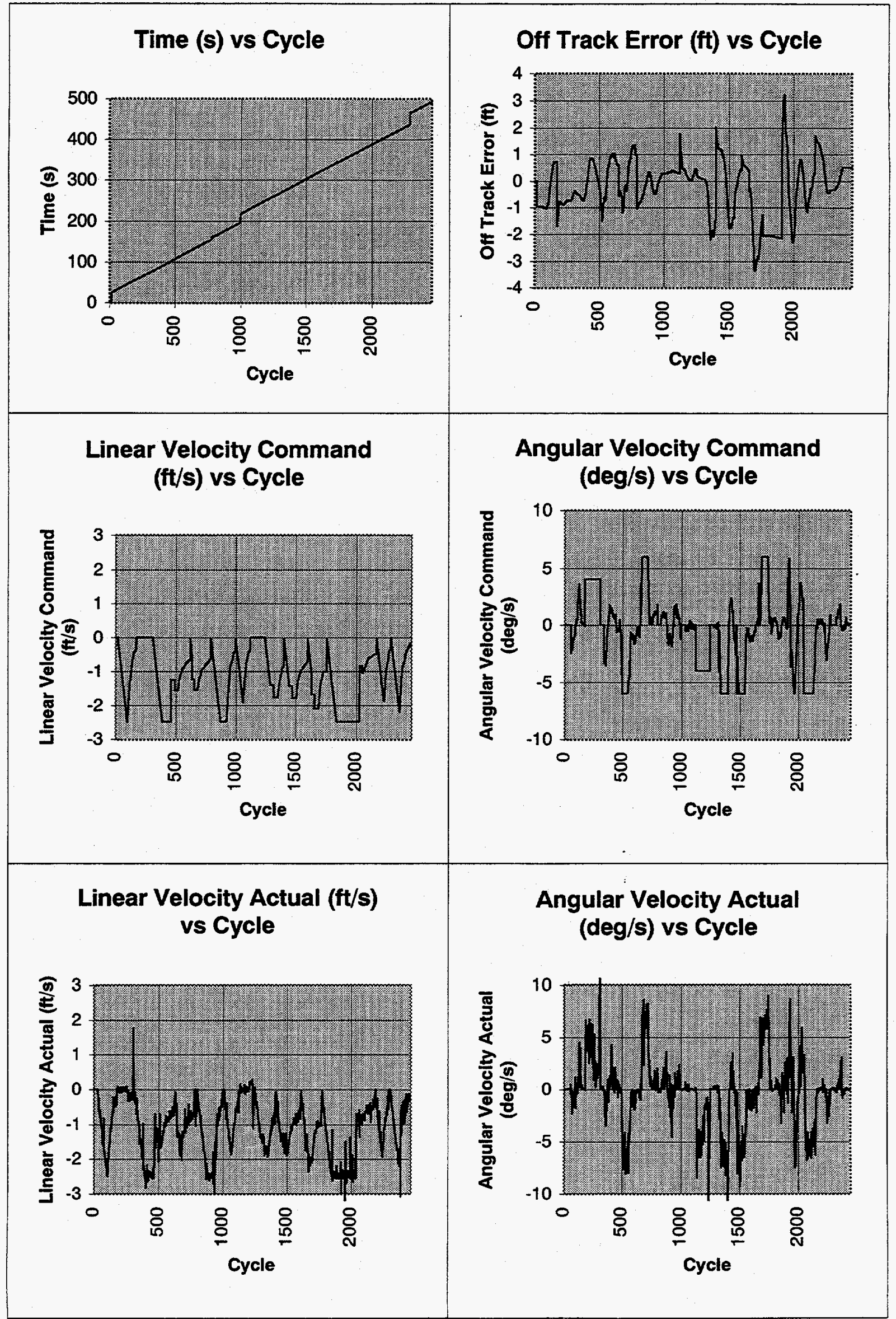




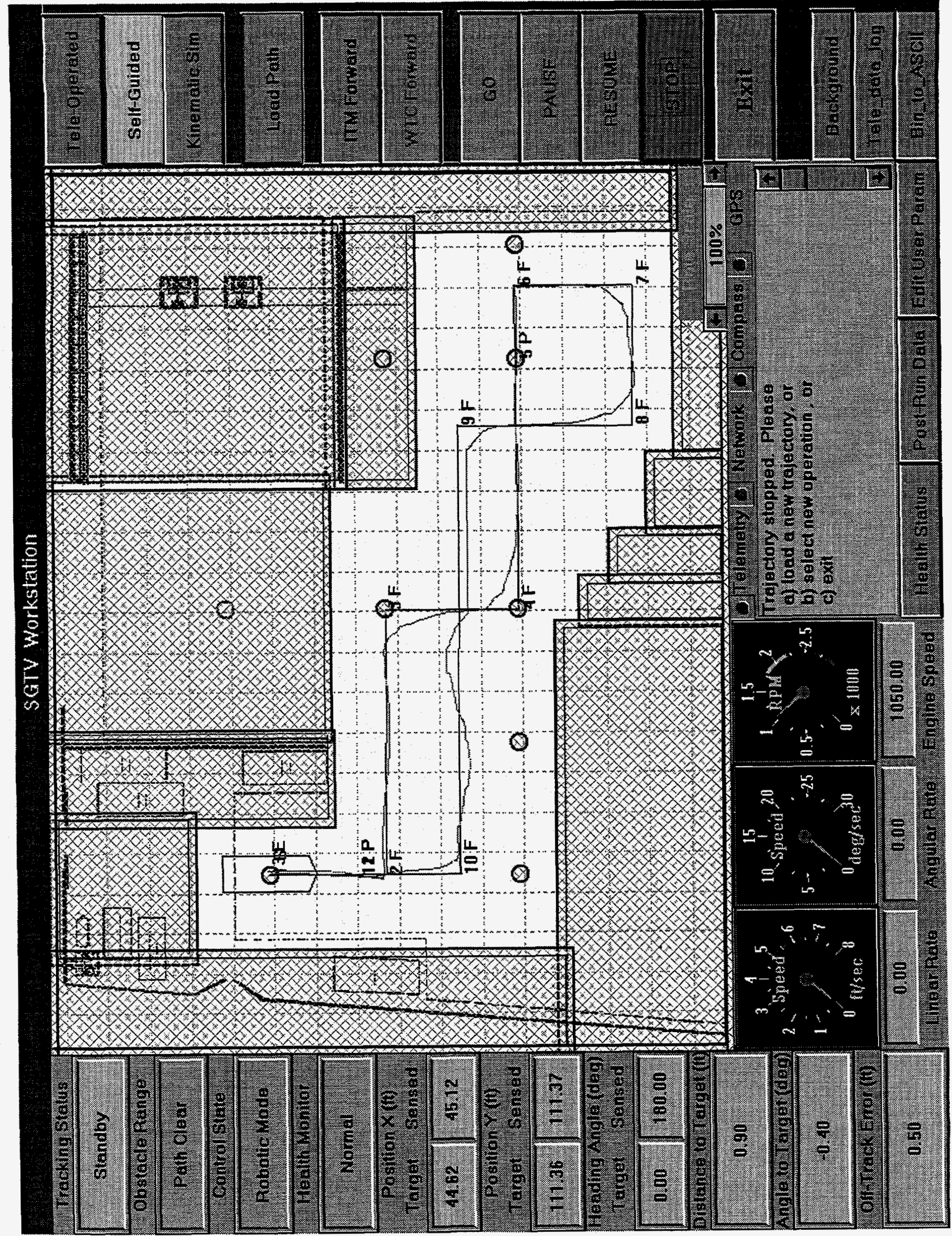




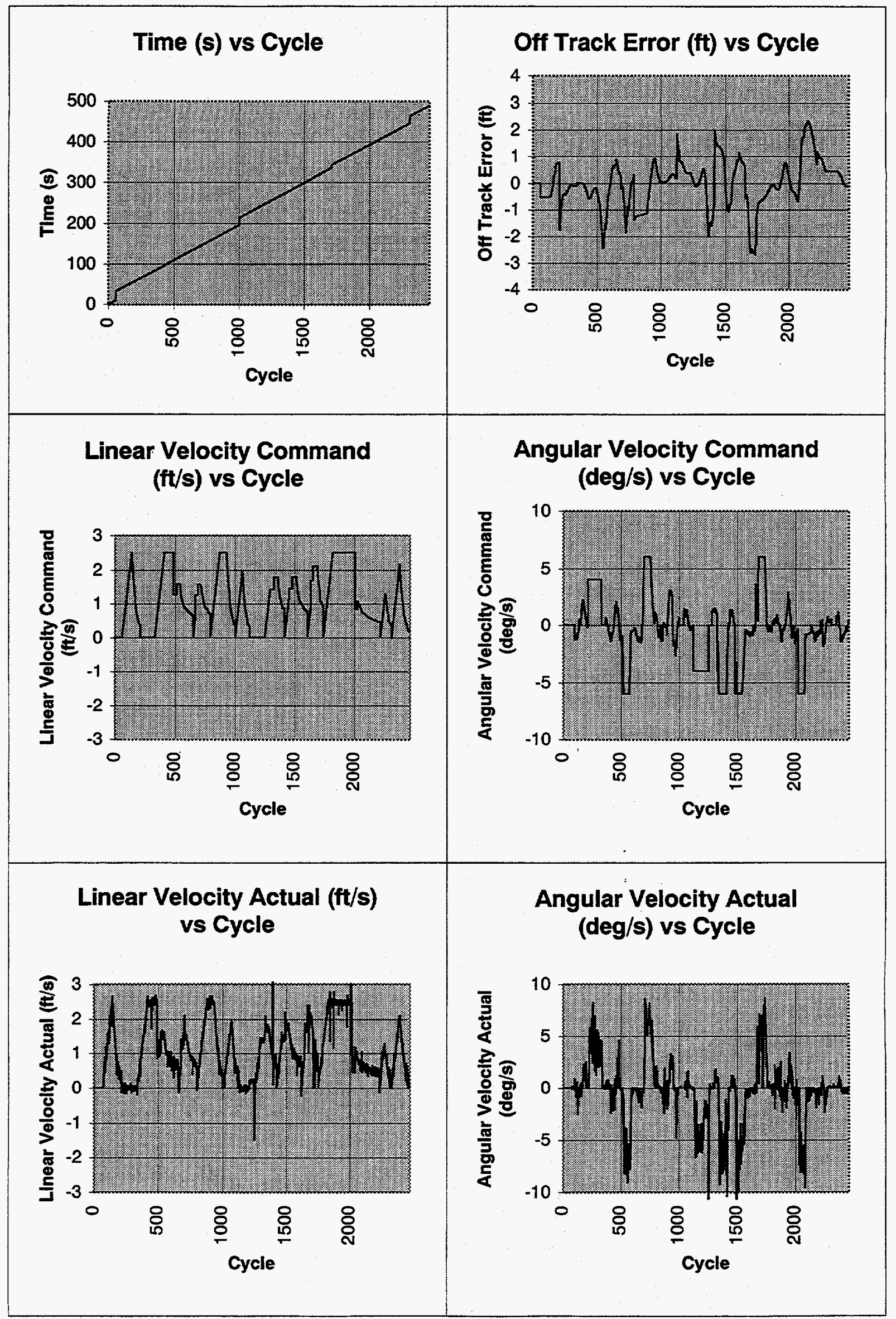




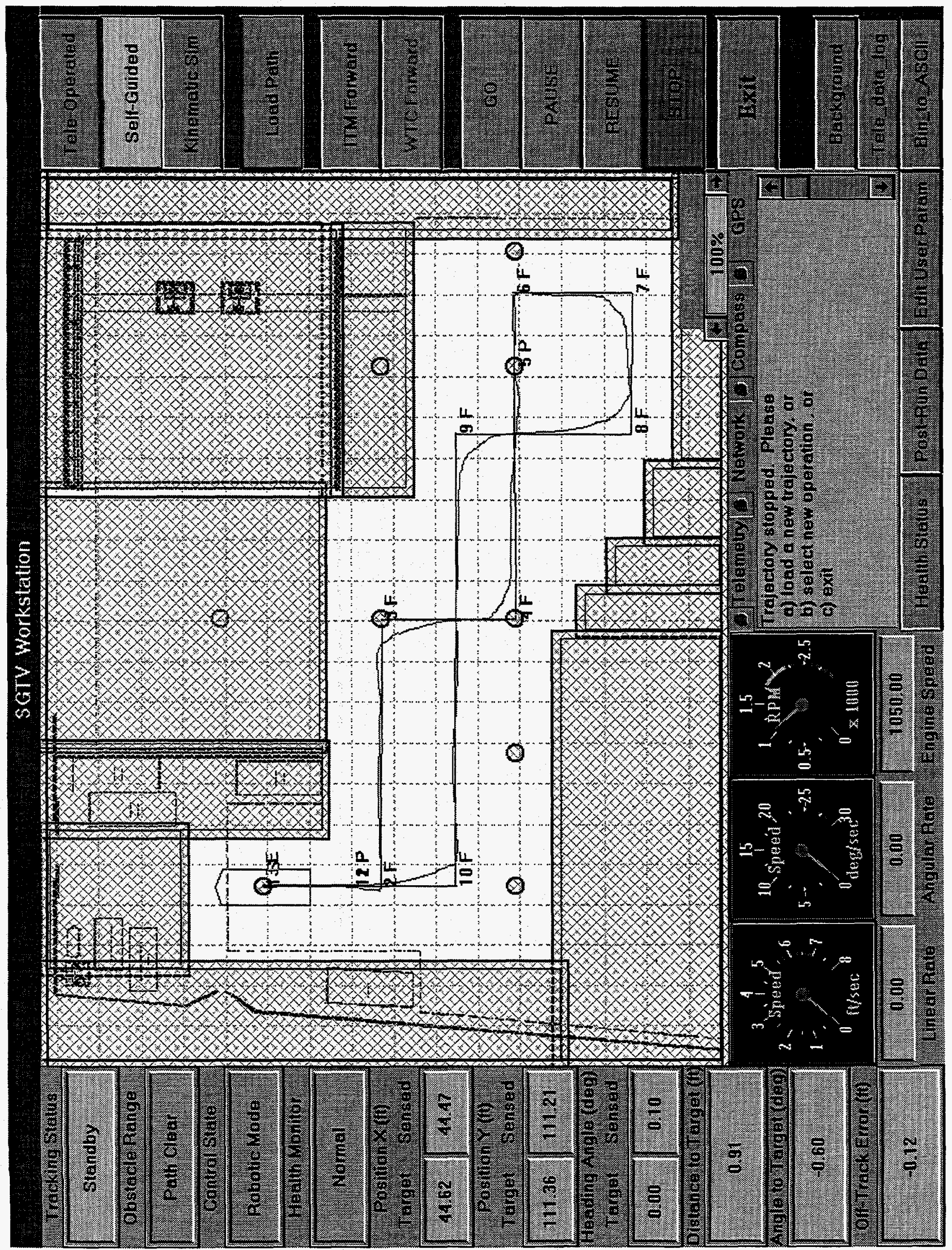




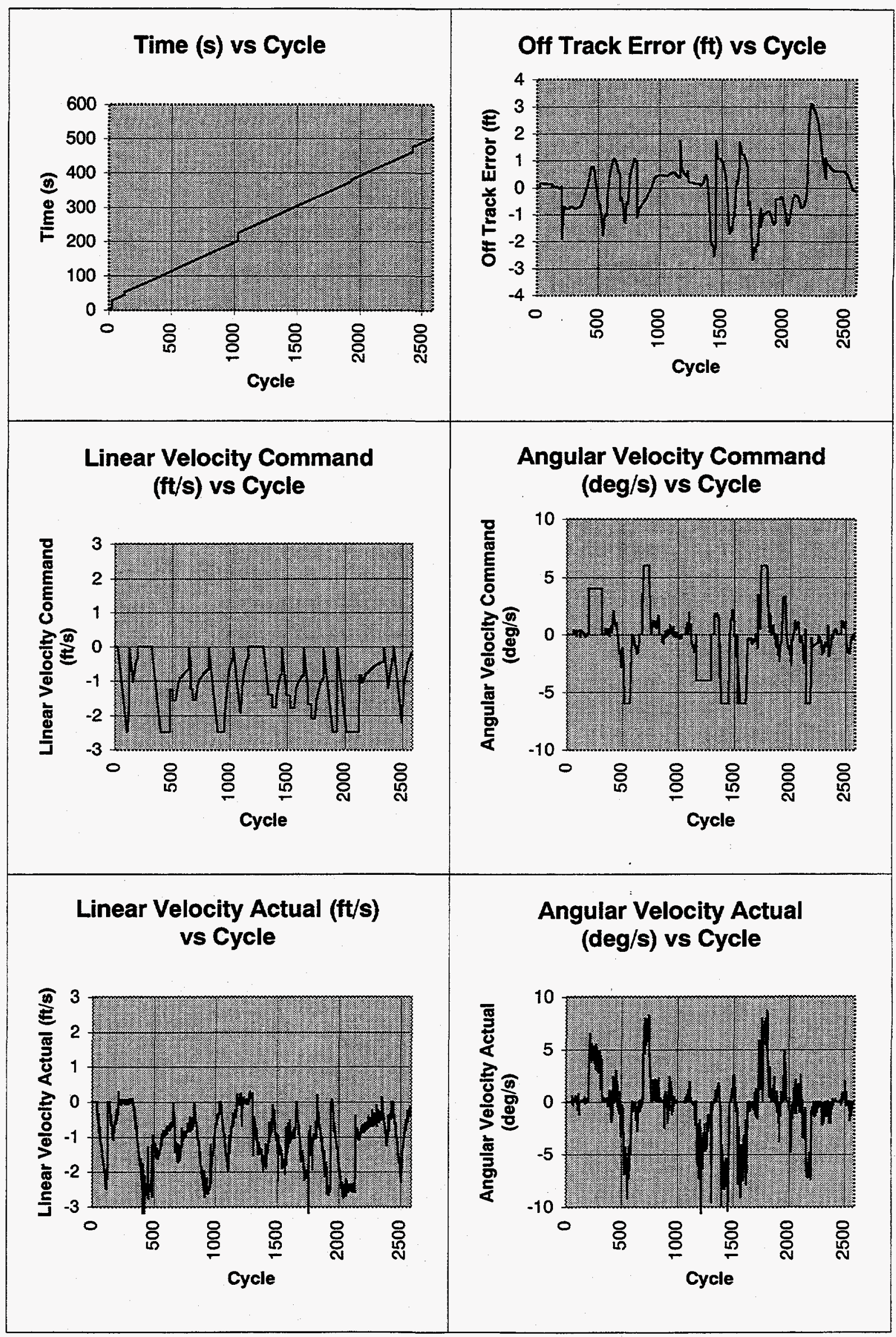




\section{SGTV Workstation}

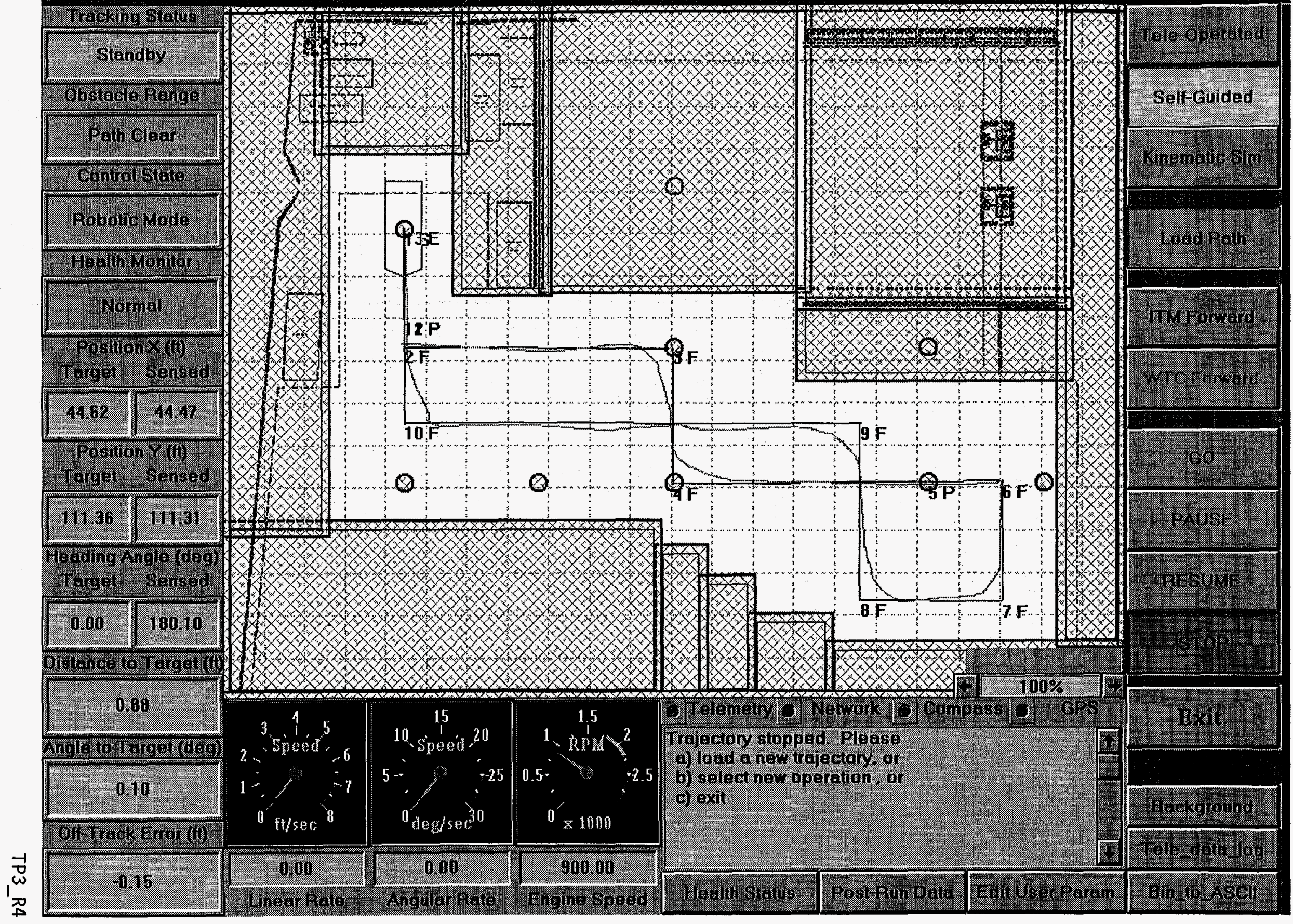




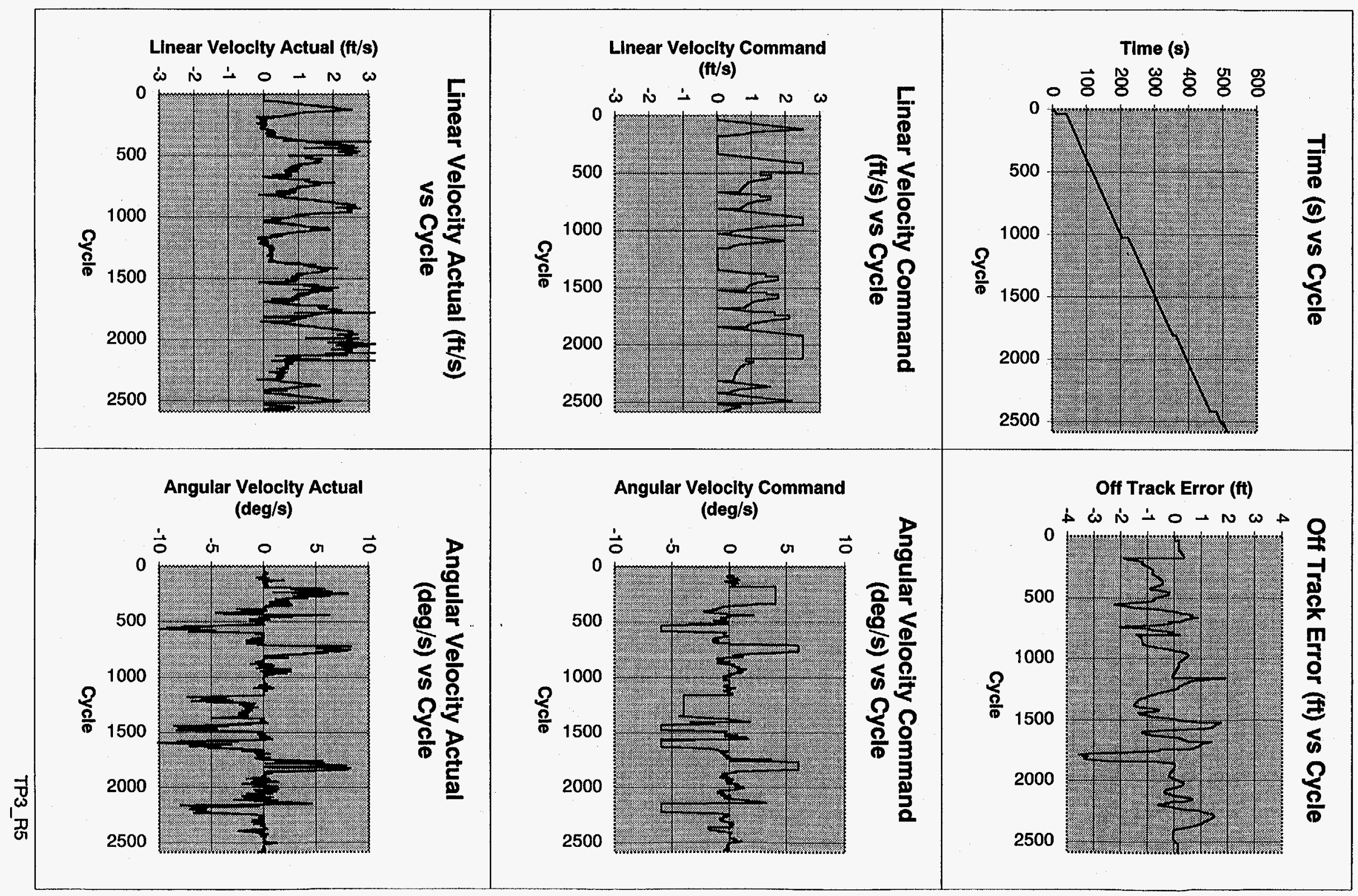




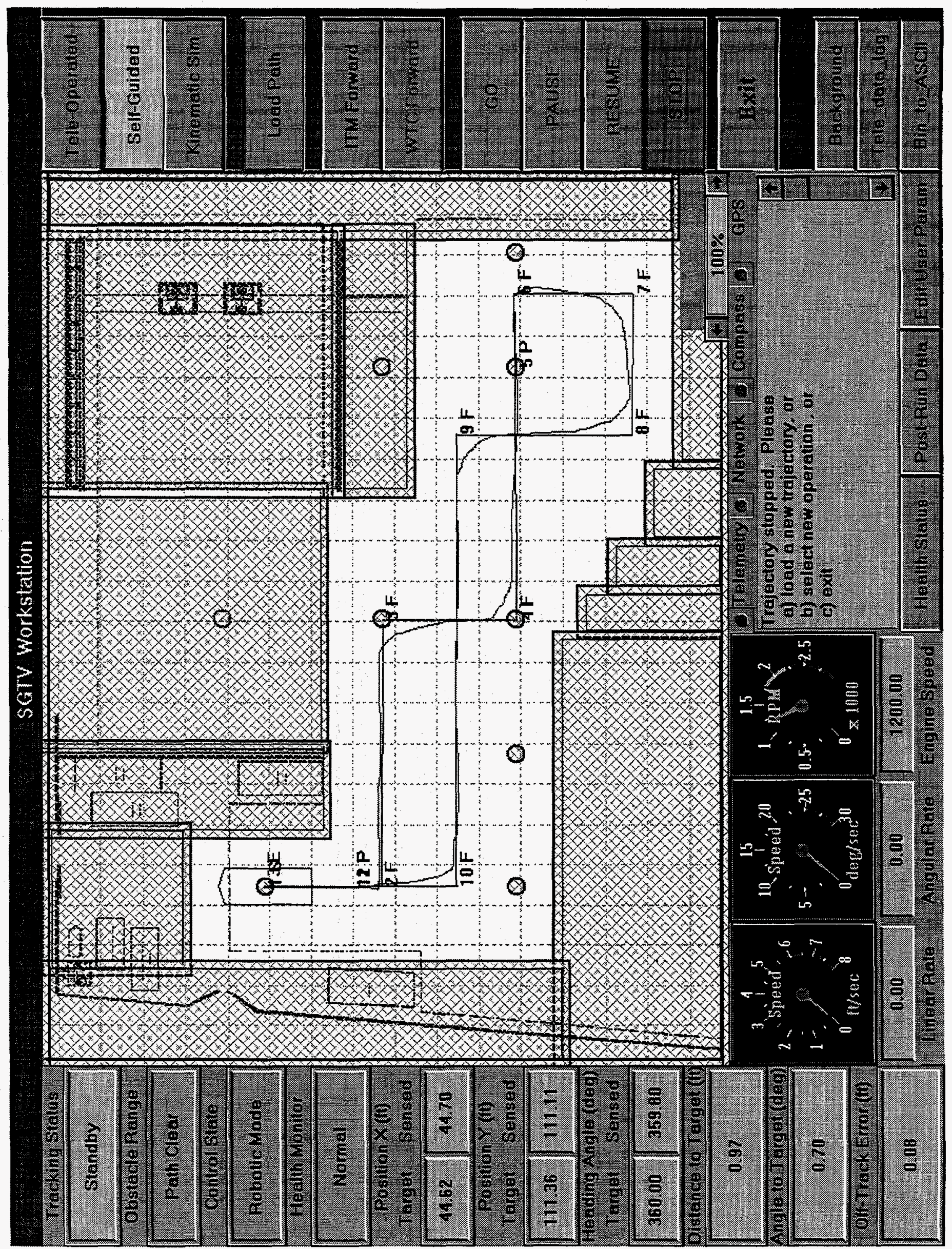




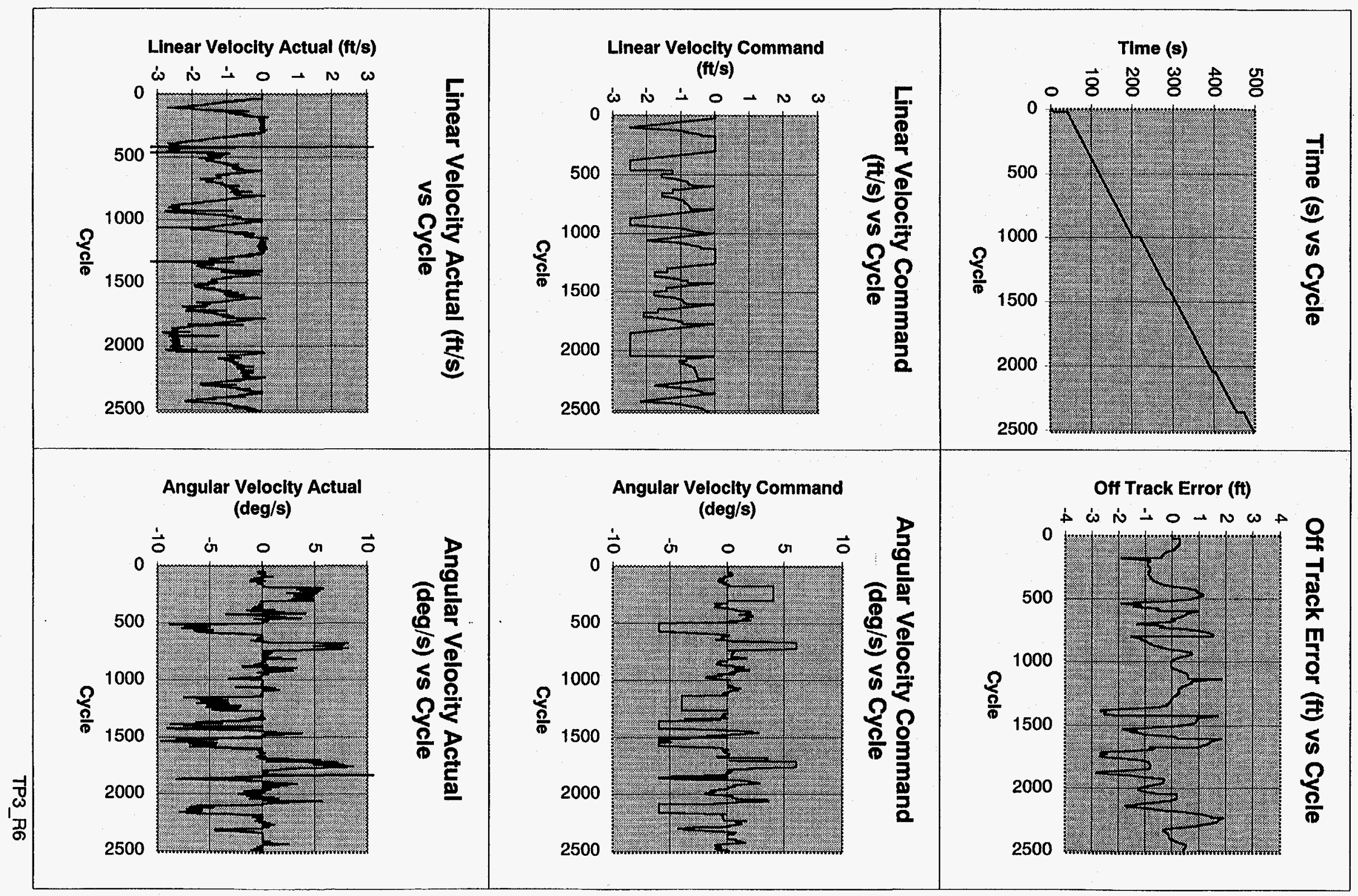


$9 y^{-} \varepsilon d 1$

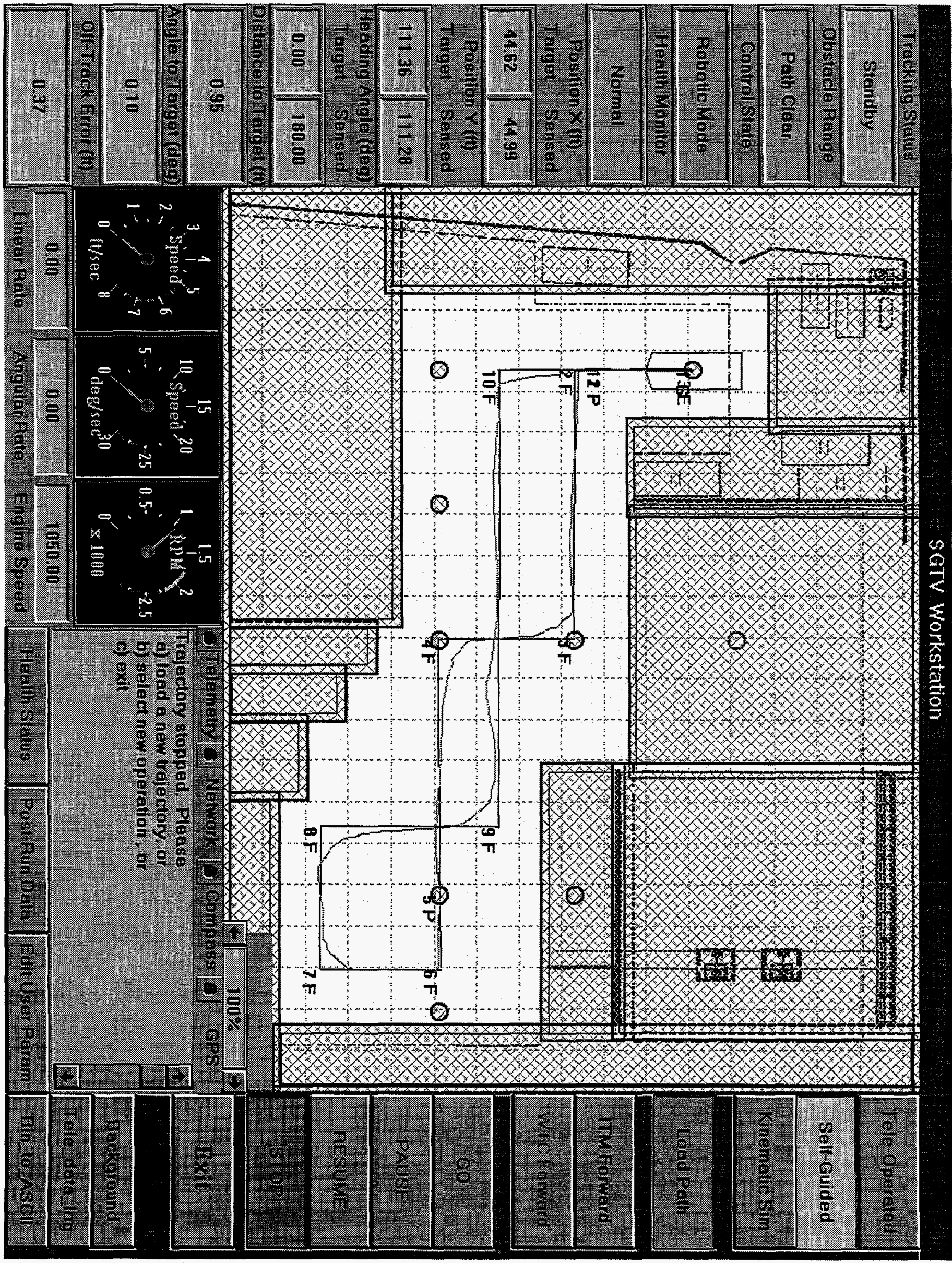




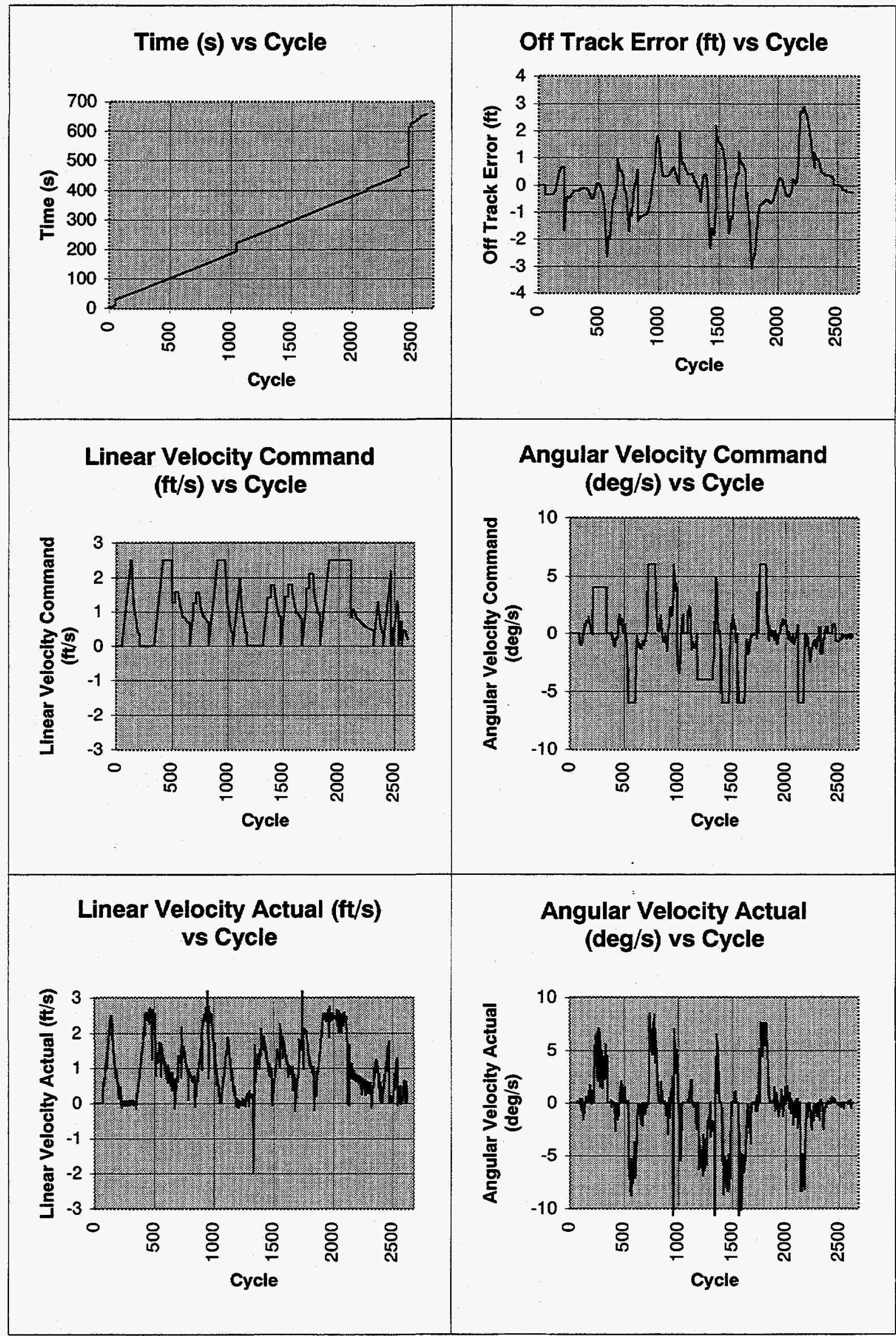


$\angle y^{-} \varepsilon d \perp$

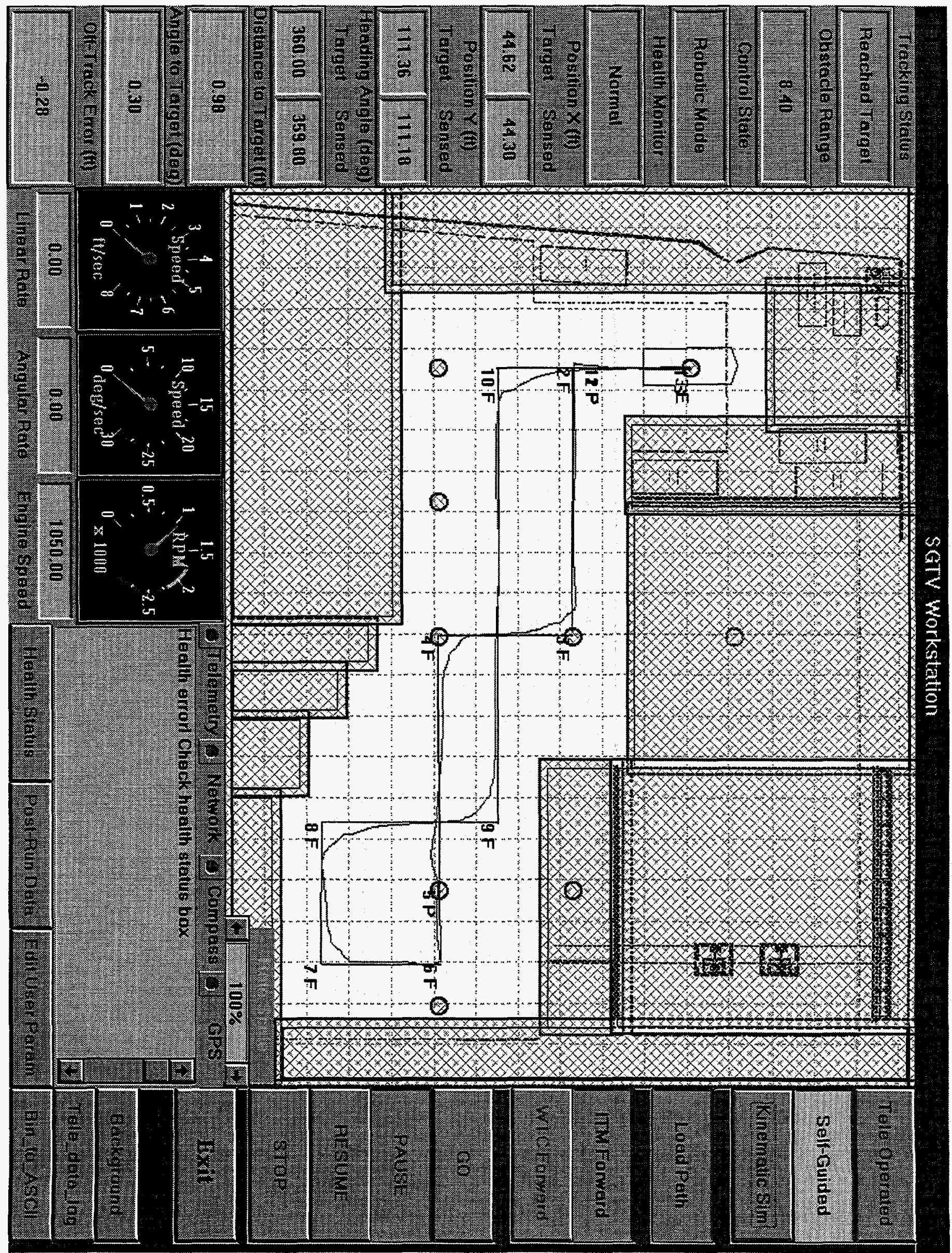




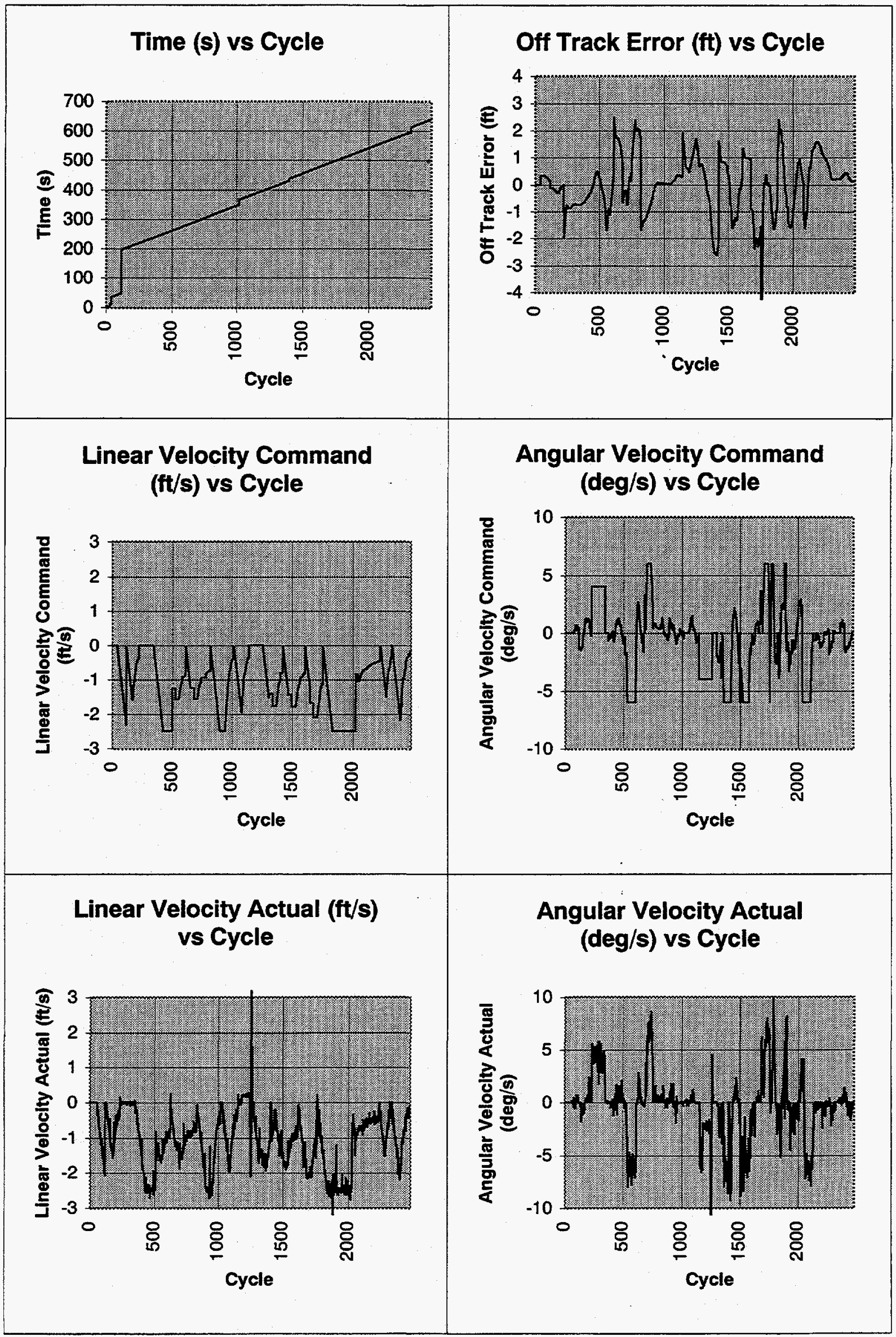




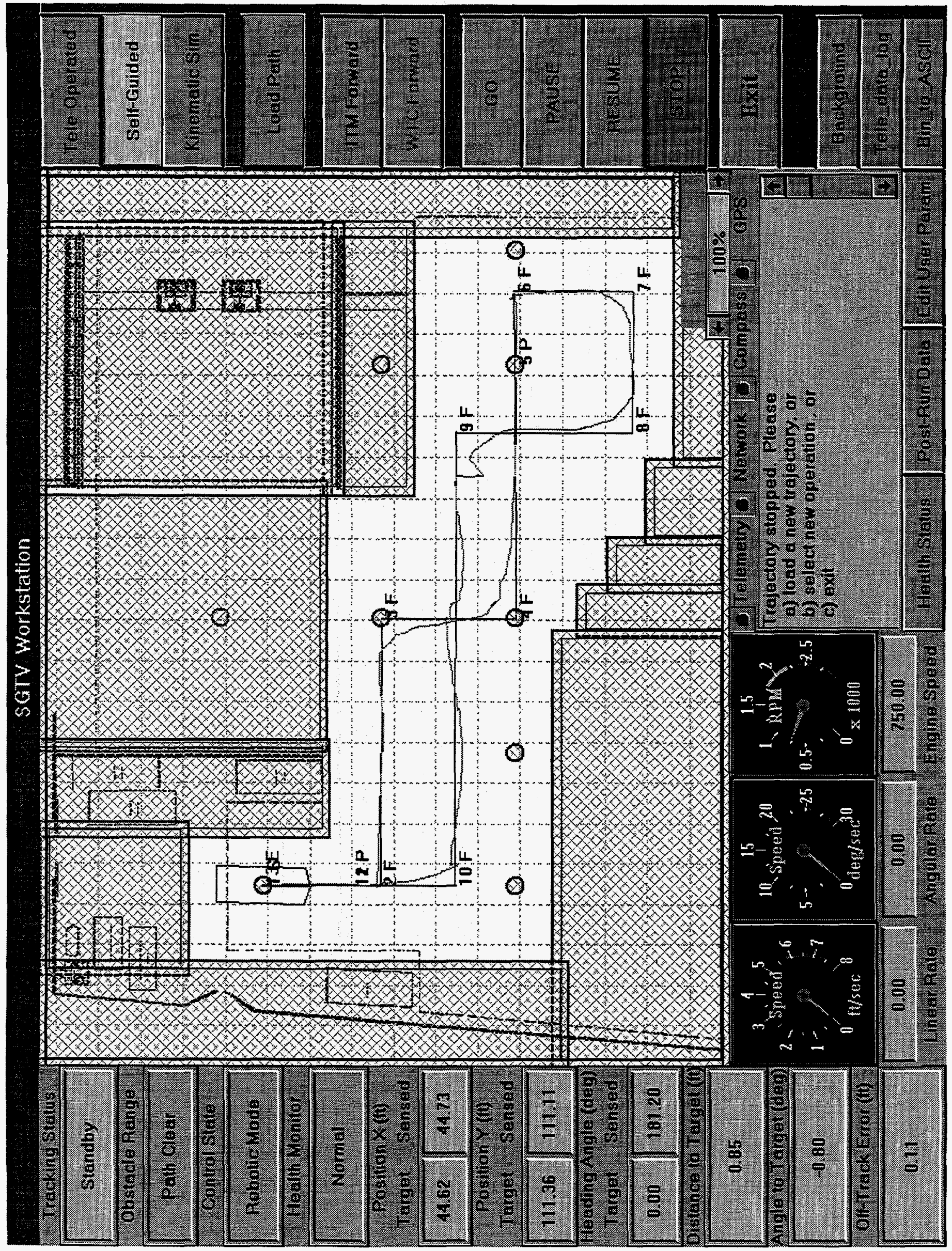




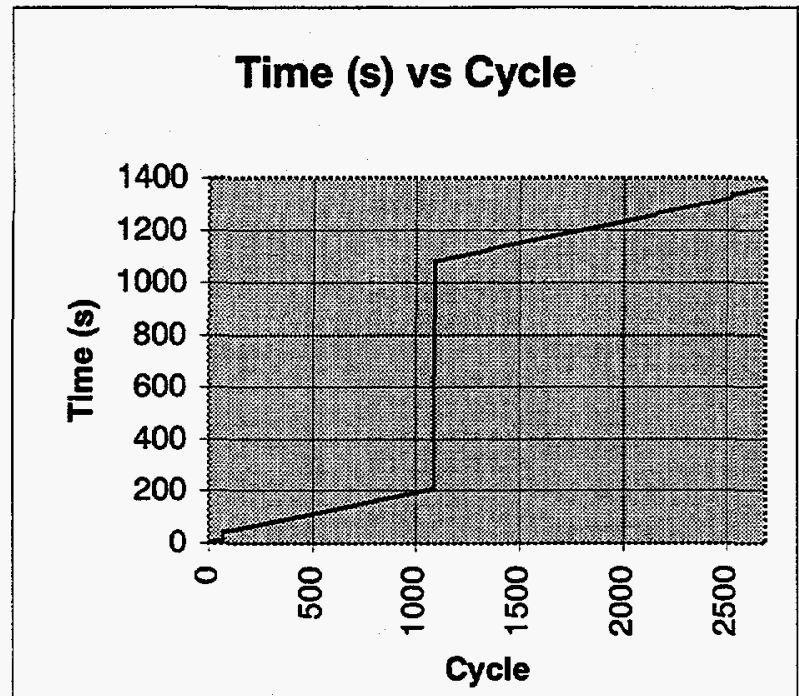

Off Track Error (ft) vs Cycle

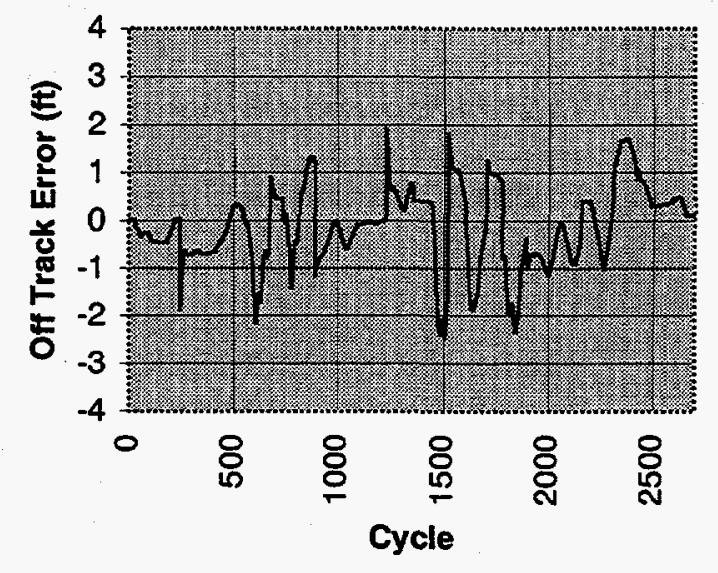

Linear Velocity Command (ft/s) vs Cycle

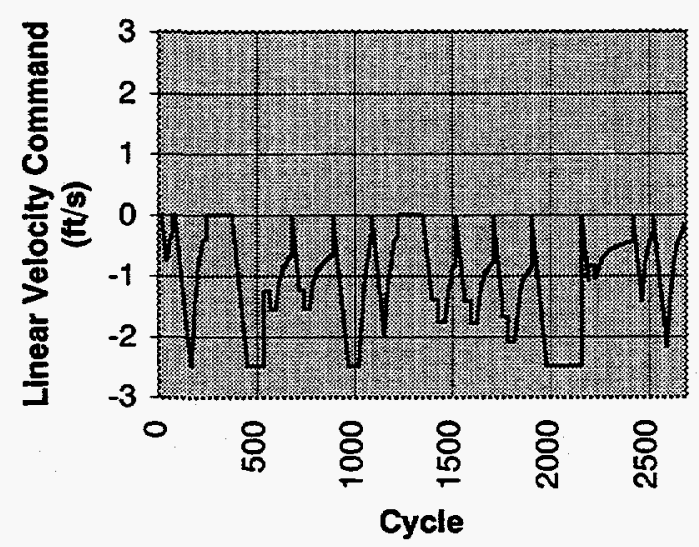

Angular Velocity Command (deg/s) vs Cycle

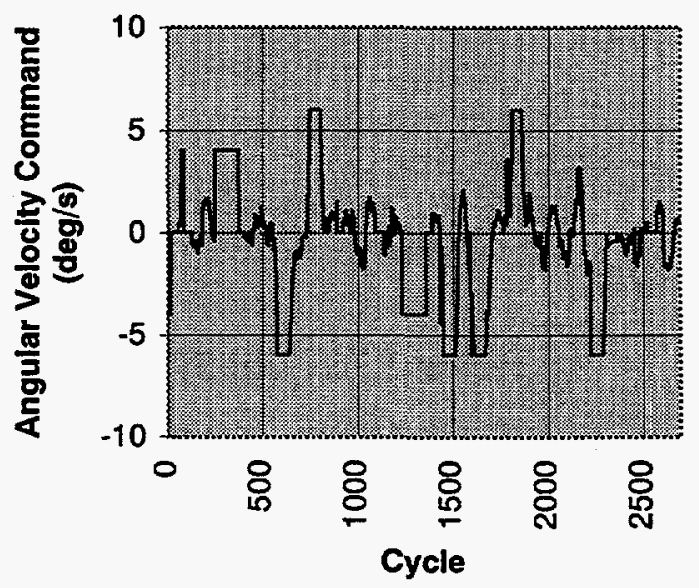

Linear Velocity Actual (ft/s) vs Cycle

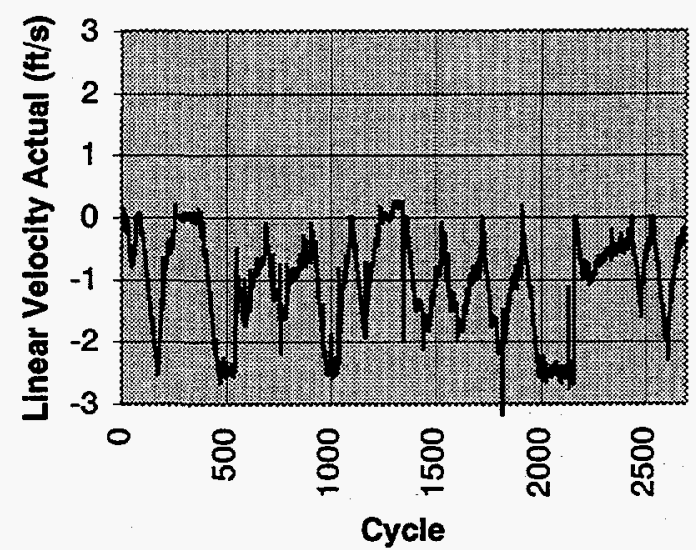

\section{Angular Velocity Actual} (deg/s) vs Cycle

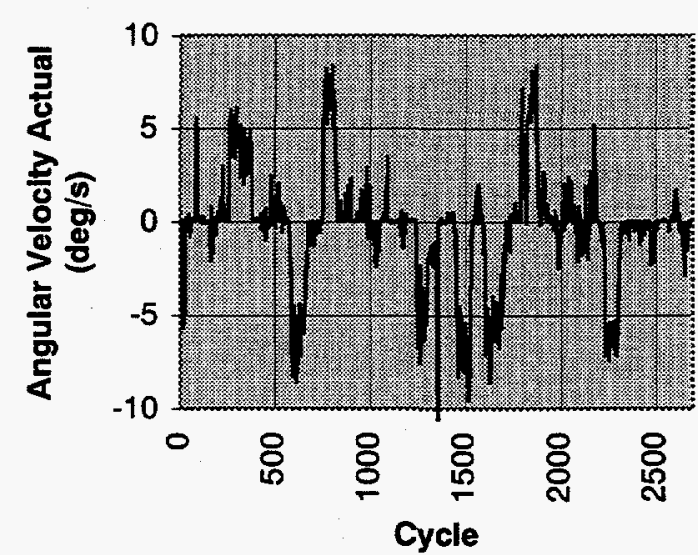




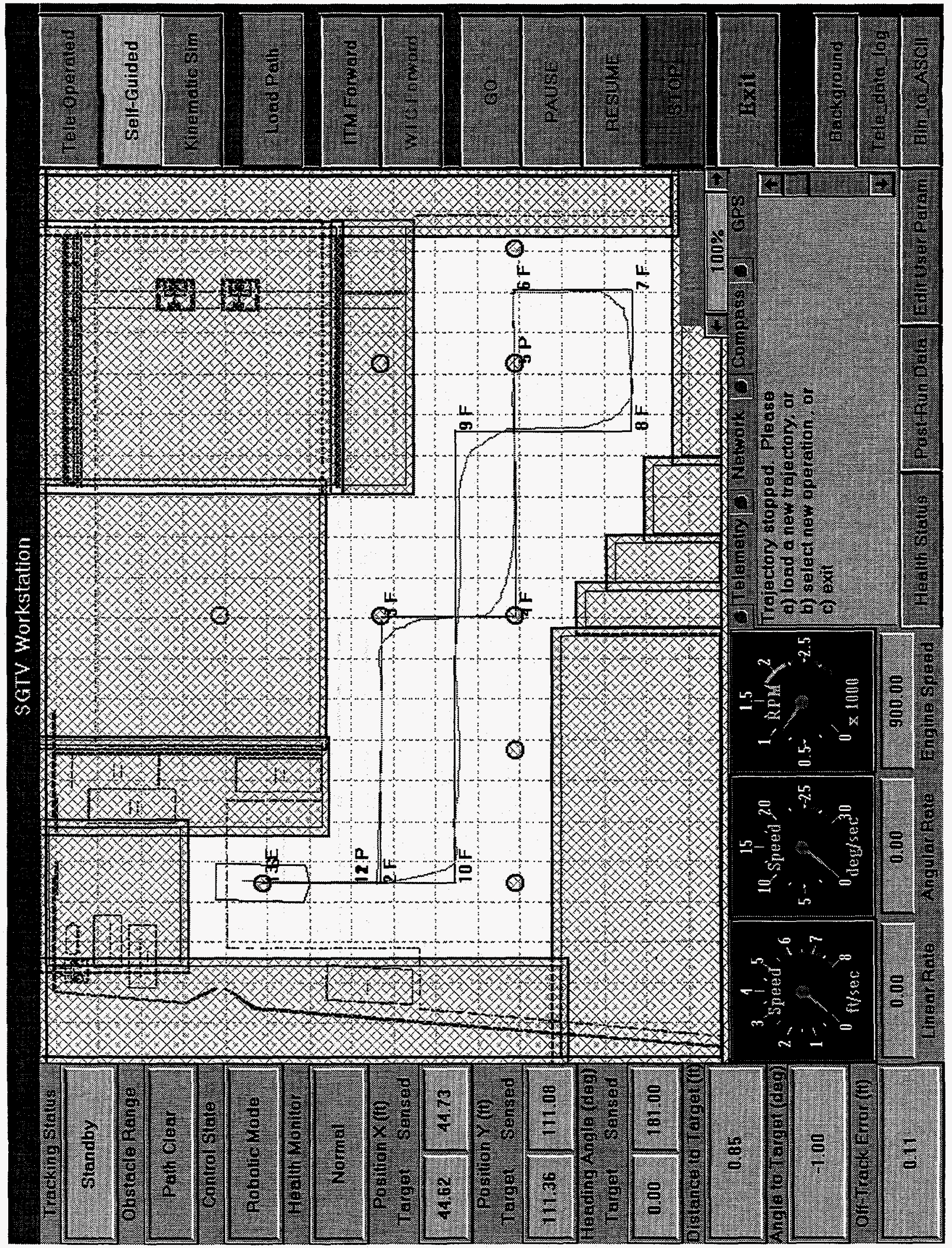




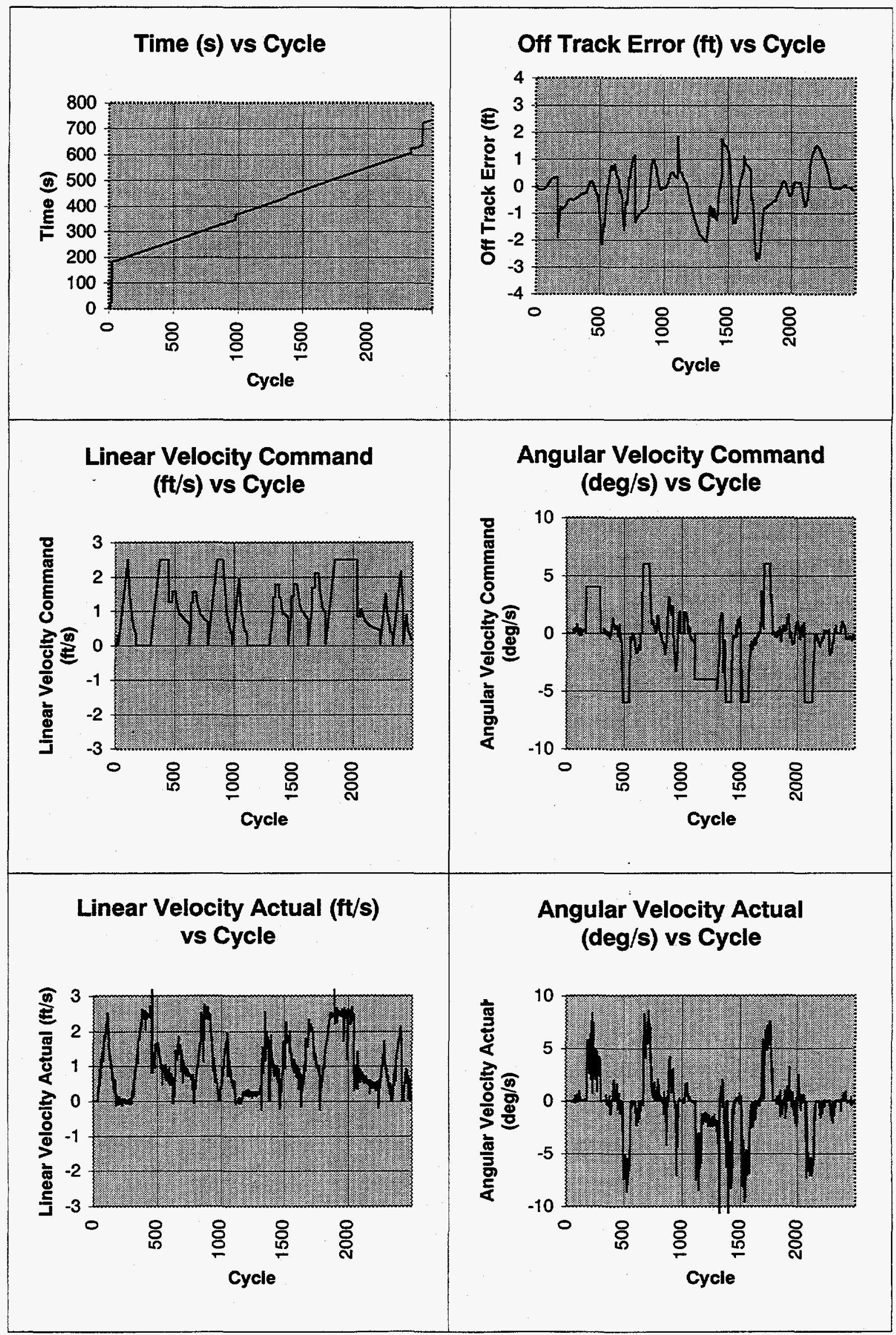




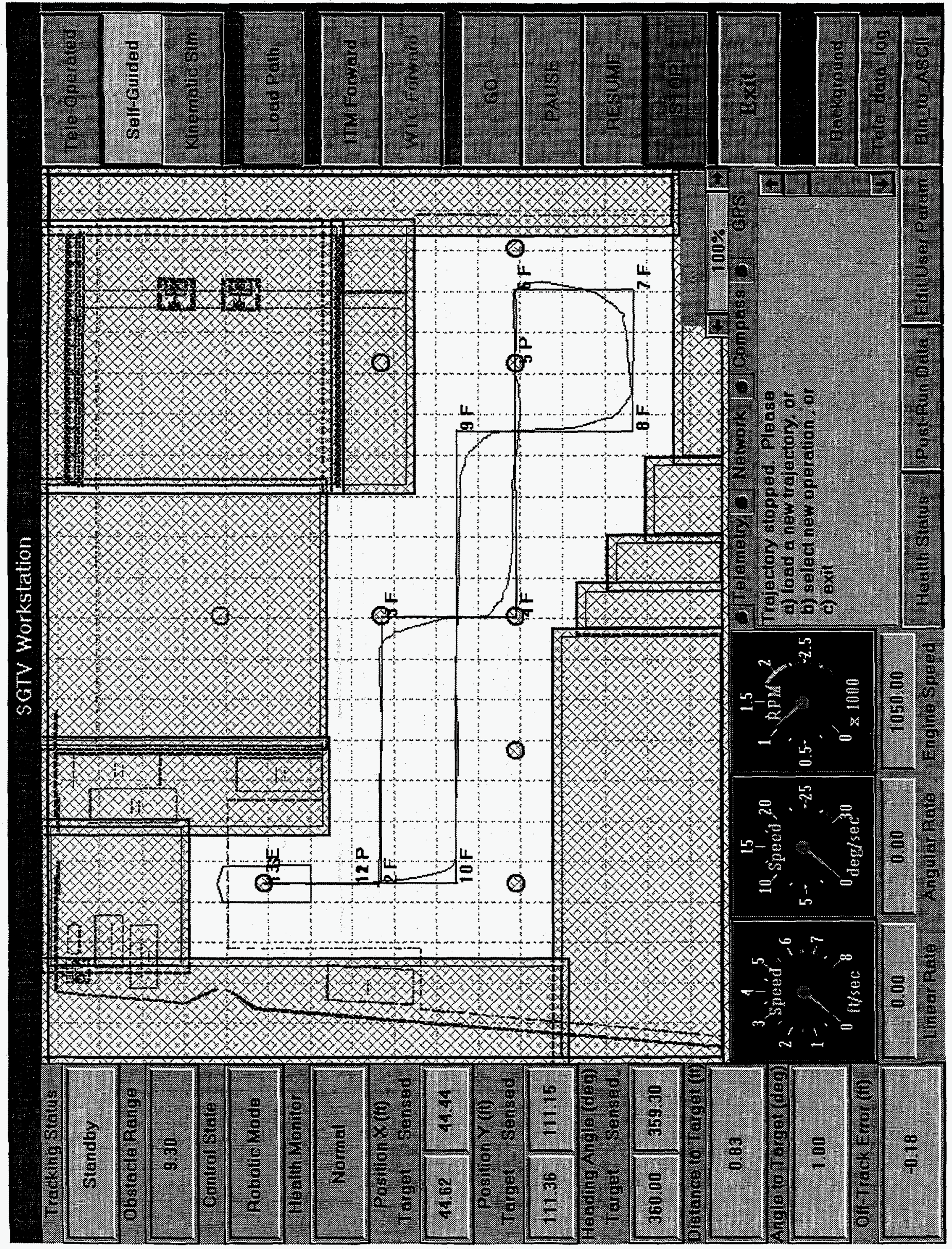

